\title{
QUANTIFICATION OF FORCES INVOLVED IN CARBOHYDRATE INDUCED ATTACHMENT OF CARCINOMA CELLS
}

\author{
Dissertation \\ for the award of the degree \\ Doctor rerum naturalium \\ of the Georg-August-Universität Göttingen \\ within the doctoral program \\ Physics of Biological and Complex Systems \\ of the Georg-August University School of Science (GAUSS) \\ submitted by \\ Andrea Mareike Cordes \\ from Papenburg
}

Göttingen 2019 
Members of the Thesis CommitTeE

Dr. Angelika Kunze,

Institute of Physical Chemistry,

Georg-August-Universität Göttingen

Prof. Dr. Claudia Steinem,

Institute of Organic and Biomolecular Chemistry,

Georg-August-Universität Göttingen

Dr. Florian Rehfeldt,

Third Physical Institute,

Georg-August-Universität Göttingen

MEMBERS OF THE EXAMINATION BOARD

Referee

Dr. Angelika Kunze,

Institute of Physical Chemistry,

Georg-August University Göttingen

$2^{\text {nd }}$ Referee

Prof. Dr. Claudia Steinem,

Institute of Organic and Biomolecular Chemistry,

Georg-August University Göttingen

FURTHER MEMBERS OF THE EXAMINATION BOARD

Dr. Florian Rehfeldt,

Third Physical Institute,

Georg-August University Göttingen

Dr. Sebastian Kruss

Institute of Physical Chemistry,

Georg-August University Göttingen

Prof. Dr. Silvio Rizzoli

Department of Neuro- and Sensory Physiology

University Medical Center Göttingen

Prof. Dr. Michael Meinecke

European Neuroscience Institute

Georg-August University Göttingen

DATE OF ORAL EXAMINATION

08.05.2019 


\section{DEClaRATION}

I, Andrea Mareike Cordes, hereby certify that my doctoral thesis entitled "Quantification of forces involved in carbohydrate induced attachment of carcinoma cells" has been written independently and with no other sources and aids than quoted.

Göttingen, 15th May 2019

Andrea Mareike Cordes 

Meiner Familie 

ABSTRACT Vital biological processes such as the adhesion of cells to their surroundings rely on short-term recognition processes, which are defined by a rapid assembly and disassembly of adhesion molecules. These processes were mostly attributed to interactions of membrane-bound proteins to complementary proteins (PPI) or carbohydrates (CPI), while direct interactions between carbohydrates (CCI) were underestimated due to their low homotypic binding affinity. However, the cellular plasma membrane offers an abundant supply of carbohydrate-containing molecules including glycosphingolipids (GSLs), which are exclusively displayed on the outer leaflet of mammalian cell membranes. GSLs are characterized by extremely high variability due to a large diversity of different carbohydrate head groups and were found to interact with high selectivity.

In this thesis, two different methods based on atomic force microscopy (AFM), namely colloidal probe microscopy (CPM) as well as single-cell force spectroscopy (SCFS), were applied to assess the impact of CCIs between the GSLs $\mathrm{G}_{\mathrm{M} 3}$ and lyso-lactosylceramide (lysoLacCer) on the initial adhesion of murine B16 melanoma cancer cells. To study the relation between CCI and tumor malignancy, B16-F1 and B16-F10 cells of the same origin but different malignancy were used. Surface-based analytical experiments including fluorescence and optical cell adhesion studies were applied to examine the distribution and organization of $\mathrm{G}_{\mathrm{M} 3}$ and lyso-LacCer in the cellular plasma membrane and in solid supported lipid membranes (SSLMs) and indicated the formation of GSL-enriched domains (GEMs). CPM employing a model membrane system allowed to examine the specific adhesion forces between $\mathrm{G}_{\mathrm{M} 3}$ and lyso-LacCer due to a full control over molecular composition in SSLMs. Our CPM results showed that strong maximum adhesion forces of approximately $100-400 \mathrm{pN}$ exist between $\mathrm{G}_{\mathrm{M} 3}$ and lyso-LacCer at contact times of 0-60 s, which were comparable to adhesion forces found between other GSLs, CPIs and even PPIs. Since we assume similar amounts of binding partners in the contact area of the glass microsphere and the cells, maximum adhesion forces obtained by CPM can be compared to force values detected by SCFS. We found that CPM observed forces were quite similar to the maximum adhesion forces obtained by SCFS at contact times shorter than $5 \mathrm{~s}(\sim 100-500 \mathrm{pN})$ before high adhesion forces of $750 \mathrm{pN}$ (B16F1) and $1800 \mathrm{pN}$ (B16-F10) were detected for contact times of 10-60 s. We propose that the CCIs between $\mathrm{G}_{\mathrm{M} 3}$ and lyso-LacCer have the potential to foster B16 cell attachment within the first seconds of adhesion. At longer contact times, associations between GSLs and adhesive molecules including proteins are presumably actively induced by cellular internal mechanisms leading to an enhanced adhesion caused by signaling processes. The stronger adhesion behavior of the invasive B16-F10 cells points at an enhanced recruitment of $\mathrm{G}_{\mathrm{M} 3}$ to the basal attachment site based possible on interactions with adhesive molecules leading to a more effective metabolism.

In this thesis, I was able to show by using a combination of tailor-made model systems and live cell studies that CCIs play a significant role in early adhesion processes of the murine B16 melanoma cancer cells. 



\section{CONTENTS}

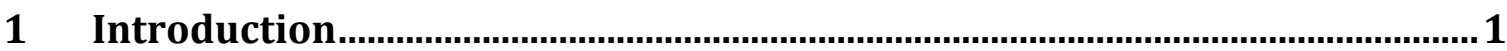

$1.1 \quad$ Melanoma skin cancer .......................................................................................... 1

1.1.1 The B16 melanoma cell line................................................................ 2

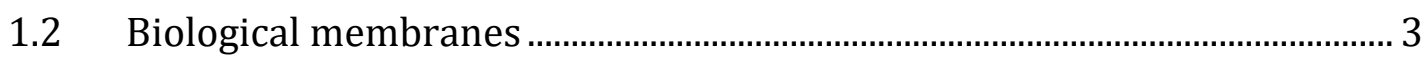

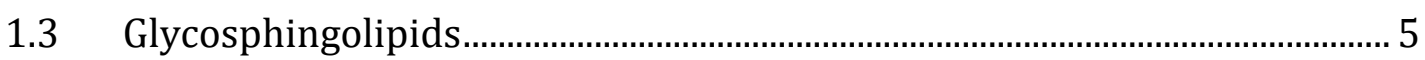

1.3.1 Conformation and organization ........................................................ 7

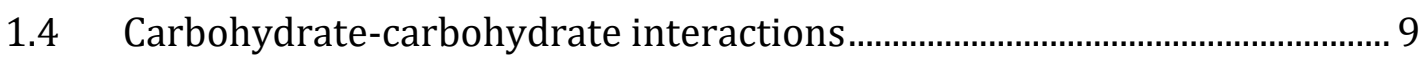

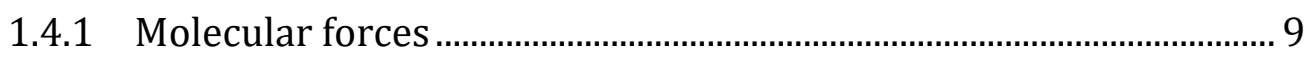

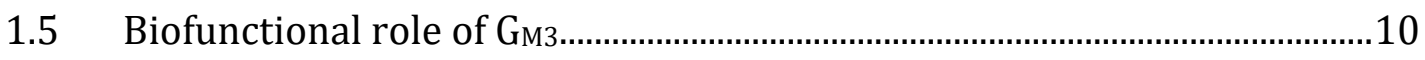

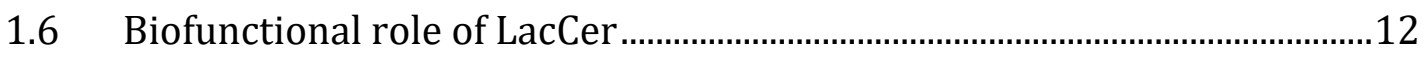

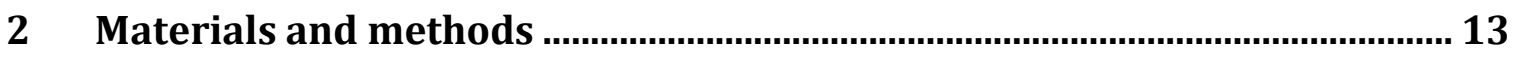

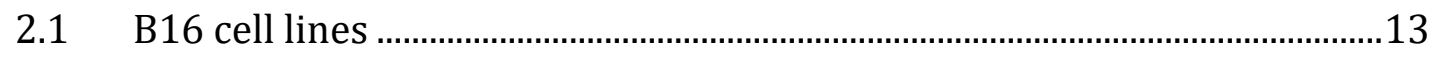

2.1.1 Cell culture conditions .................................................................15

2.1.2 Staining and functionalization ..........................................................16

2.1.2.1 Immunostaining protocols..............................................................16

2.1.2.2 Functionalization protocols.............................................................18

2.2 Lipidchemical experimental procedures ......................................................19

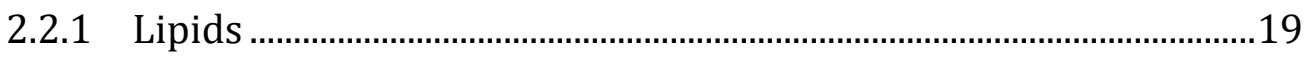

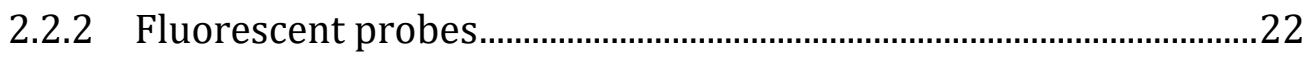


2.2.3 Preparation of small unilamellar vesicles ..........................................23

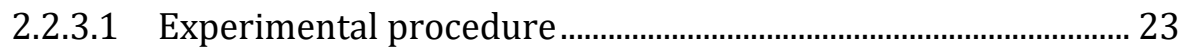

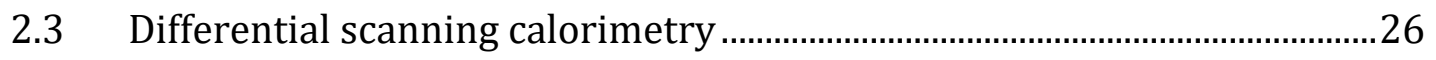

2.3.1 Setup and measuring principle..............................................................26

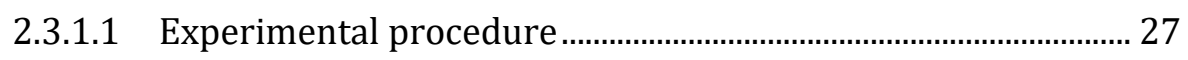

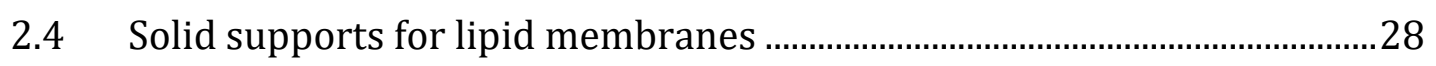

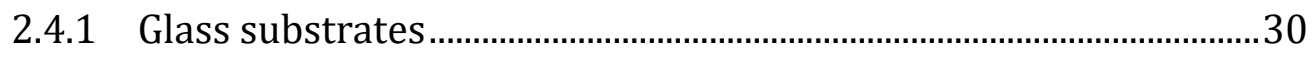

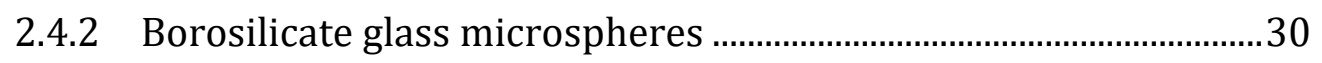

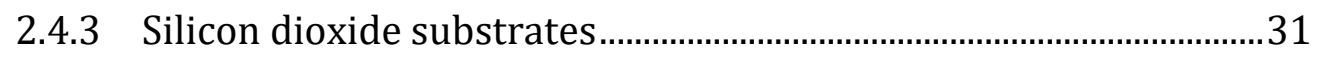

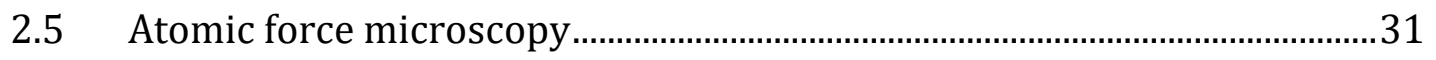

2.5.1 Setup and measuring principle...............................................................32

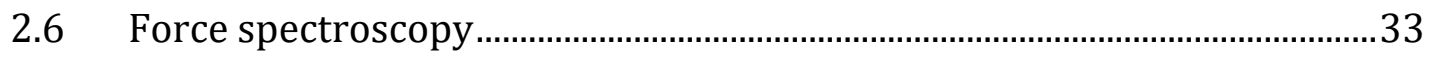

2.6.1 Single-cell force spectroscopy ……...................................................35

2.6.1.1 Experimental procedure .................................................................. 35

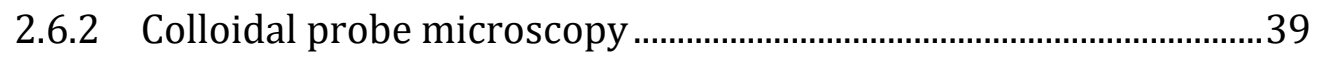

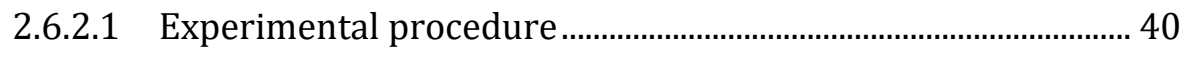

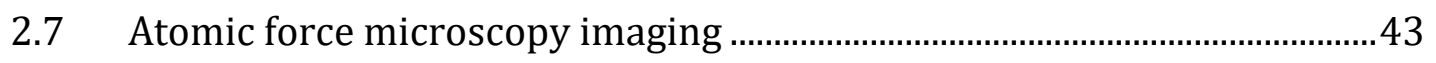

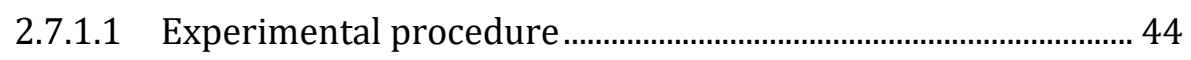

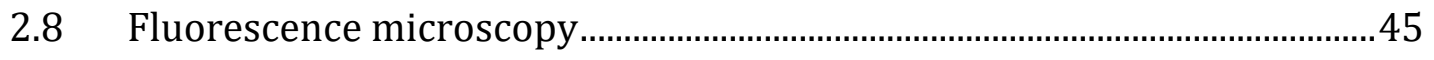

2.8.1 Epifluorescence microscopy.............................................................. 46

2.8.1.1 Experimental procedure ................................................................. 47

2.8.2 Confocal laser scanning microscopy .................................................48

2.8.2.1 Experimental procedure .............................................................. 49

2.8.3 Fluorescence recovery after photobleaching....................................49

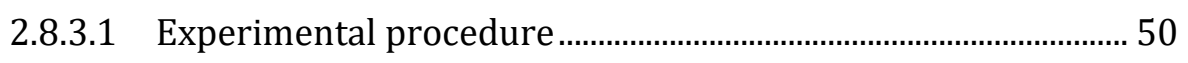

2.8.4 Total internal reflection fluorescence...............................................51

2.8.4.1 Experimental procedure ................................................................. 52 
2.8.5 Image-based cytometry ............................................................................

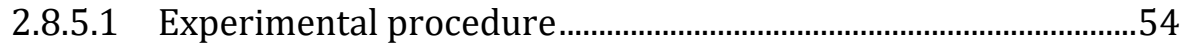

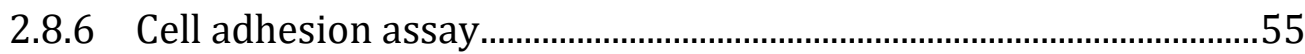

2.8.6.1 Experimental procedure ..................................................................55

2.9 Reflection interference contrast microscopy ….............................................56

2.9.1.1 Experimental procedure ................................................................5

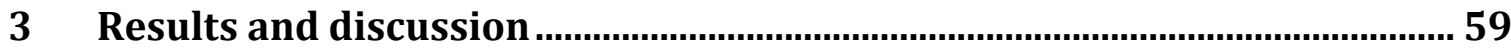

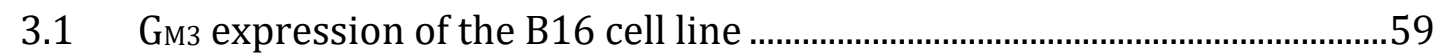

3.2 Cell adhesion properties of the B16 cell line ...................................................65

3.3 Development of a model membrane system .................................................71

3.4 Single-cell force spectroscopy of B16 cells...........................................................

3.4.1 Quantification and validation of the carbohydrate-carbohydrate

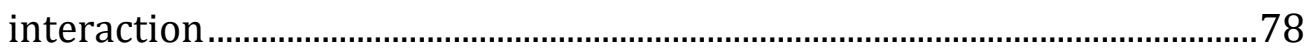

3.5 Force spectroscopy of model membranes ........................................................87

3.5.1 Quantification and validation of the carbohydrate-carbohydrate

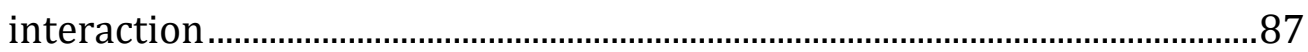

3.6 Carbohydrate-carbohydrate adhesion forces ............................................94

4 Conclusion

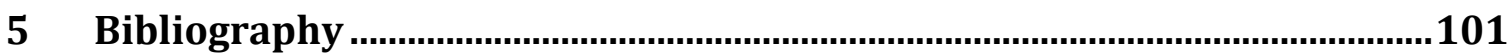

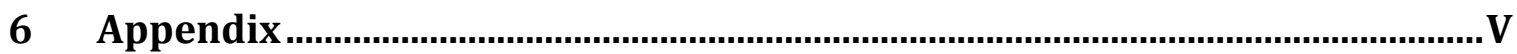

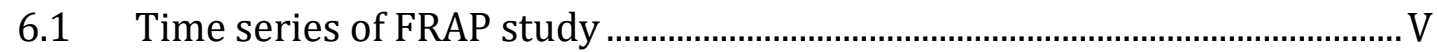

6.2 Topographical maps (AFM, tapping mode) ...................................................... VI

6.3 Distribution of maximum adhesion forces of B16-F1 .................................VII

6.4 Distribution of maximum adhesion forces of B16-F1 ............................... VIII

6.5 Distribution of maximum adhesion forces of B16-F1 ....................................

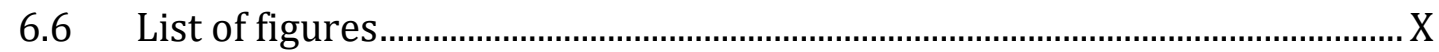

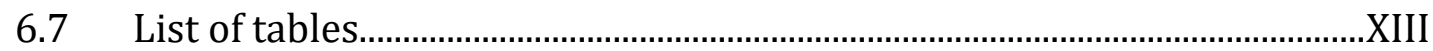


6.8 List of symbols and abbreviations ................................................................ 


\section{INTRODUCTION}

In this thesis, the impact of carbohydrate-carbohydrate interactions between the glycosphingolipids $\mathrm{G}_{\mathrm{M} 3}$ and lyso-lactosylceramide on the initial adhesion of cancer cells was investigated using the $\mathrm{G}_{\mathrm{M} 3}$-expressing murine B16 melanoma cancer cell lines B16-F1 and B16-F10. The B16 cells were used as a model system for melanoma skin cancer cells showing different metastatic behaviors. Cells studies were complemented by model membrane studies based on $\mathrm{G}_{\mathrm{M} 3}$ - or lyso-lactosylceramidecontaining solid supported lipids membranes. The following section is intended to give a brief insight into the malignant cell line and to provide information on the interaction between glycosphingolipids on vital processes.

\subsection{MELANOMA SKIN CANCER}

Cancer in general is described as an abnormal change in endogenous cells characterized by an uncontrolled growth and division process. The resulting tumor has the ability to penetrate and destroy the surrounding tissue as a consequence of nutrient removal for example. Whereas a primary tumor is defined as a tumor growth restricted to the original anatomical site, secondary tumors are characterized as a more malignant form of tumors, which spread as so called metastates to other organs throughout the body through the invasion of the blood and lymph channels. ${ }^{1}$

Skin cancer is one of the most common types of cancer in the world ${ }^{2}$ and can be differentiated into non-melanoma and melanoma skin cancer. The latter belongs to the malignant form of cancer and can arise particularly after intense UV exposure and 
recurring sunburns. ${ }^{3}$ The malignant melanoma originates in the melanocytes, which are skin cells producing the brown-colored pigment melanin giving the skin its specific tan or brown coloration. ${ }^{2}$ Malignant melanoma accounts for about $4 \%$ of all malignant neoplasms and for about $1 \%$ of all cancer deaths. ${ }^{4}$ For 2019 , the AMERICAN CANCER SOCIETY $^{2}$ predict approximately 96,500 new cases of malignant melanoma in the United States. In line with that, the RoBerT KoсH INSTITUTE 4 has recorded a steady increase in new cases in Germany since the 1970s and estimates the annual rate of malignant melanoma diagnoses at about 21,200 .

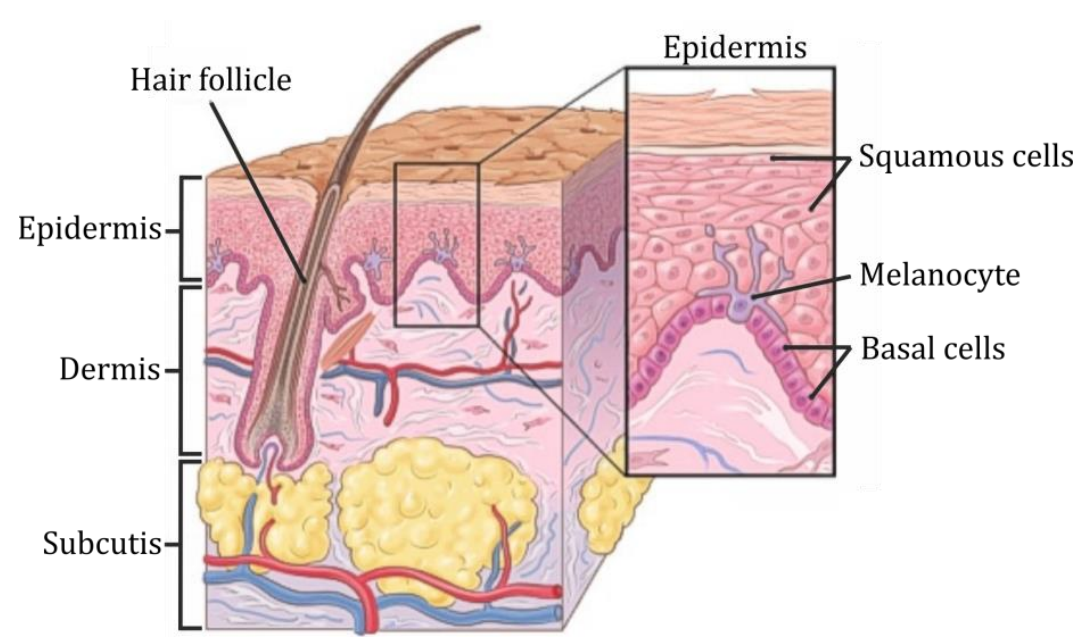

Figure 1.1: Schematic representation of the three skin layers referred to as epidermis, dermis and subcutis. ${ }^{2}$ The epidermis forms the outer layer of the skin functioning as a shield against the external environment and contains. among others. the squamous and basal cells as well as melanocytes, which produces the brown-colored pigment melanin.

\subsubsection{THE B16 MELANOMA CELL LINE}

Within this project, the Gм3-overexpressing murine B16 melanoma skin cancer cell lines B16-F1 and B16-F10 were used as a model system for melanoma cancer characterized by a different degree of malignancy. The cell lines were originally derived from a naturally grown tumor cell, which was discovered in the skin tissue behind the ear of the syngeneic mouse species C57BL/6 in 1954. To obtain low or high metastatic behaviors, FIDLER ${ }^{5,6}$ isolated the B16 melanoma tumor cells and allowed them to grow to confluency in cell culture. Afterwards the cells were injected intravenously into new syngeneic mice where particularly invasive cells populated the lungs of these mice. By repeating this procedure, the number of 
experimental malignant lung tumor colonies increased ${ }^{5-7}$ and thus enabled the acquisition of low or high metastatic cell lines. In contrast to the F1 variant, which is characterized by its rather low tendency for metastasis, the B16-F10 cell line was cultivated in a way that a high degree of metastasis could be obtained. ${ }^{6}$

The different metastasis potential of the B16-F1 and B16-F10 cells was proven by studies by FIDLER ${ }^{5}$ and POSTE${ }^{8}$, amongst others, who injected cells of the above mentioned B16 cell variants into the veins of mouse tails. Those experiments showed that the B16-F10 cells produced a higher number of foci in the lungs of the mice compared to the B16-F1 cells, which generated the lowest amount of foci. Further studies demonstrated a doubling time of 22 hours for the B16-F1 cells compared to a doubling time of 17 hours for the even more metastatic F10 variant during cultivation and preparation steps ${ }^{6,7}$ leading to a quicker confluency for B16-F10. However, both cell lines do not significantly differ regarding their shape. As shown in Figure 1.2, both adherently growing carcinoma cell lines are characterized by a spindle-shaped morphology.
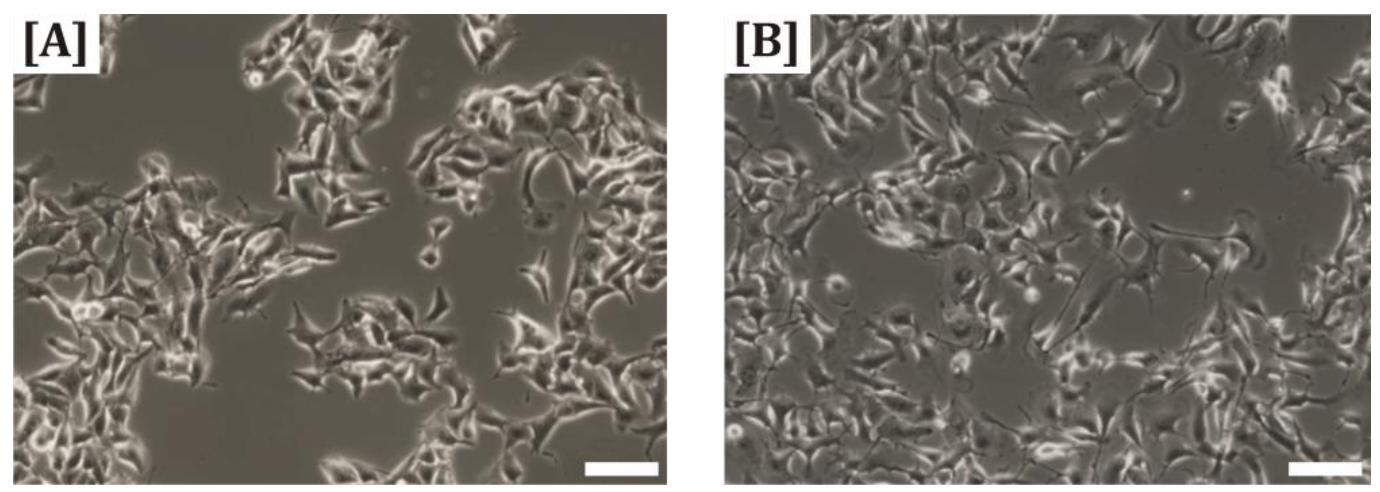

Figure 1.2: Phase-contrast micrographs of sub-confluent grown B16-F1 [A] and B16-F10 [B] cells for $48 \mathrm{~h}$ in in culture treated plastic dishes, sharing a similar spindle-shaped morphology. Cell seeding density: 75,000 cells. Scale bars: $100 \mu \mathrm{m}$.

\subsection{BIOLOGICAL MEMBRANES}

In the 1970s, SingER and NICOLSON ${ }^{9}$ introduced the fluid mosaic membrane concept as a first model of the cellular plasma membrane. According to this model, membranes, which are only a few nanometer thick, are composed of lipid molecules forming homogenous two-dimensional fluid structures in which diverse membrane proteins are embedded or associated.1,10 In the current literature, the fluid mosaic model of membrane structures is widely debated. Starting from the observation that 
glycolipids like glycosphingolipids (GSLs) are targeted to the apical cellular membrane of epithelial cells ${ }^{11-13}$, a number of studies revealed that not only the composition of the membrane components varies greatly for different membranes but also that the two monolayer leaflets of the membrane bilayer show an asymmetrically distribution of the incorporated lipids. More specifically, phosphatidylethanolamine, phosphatidylserine and phosphatidylinositide are mainly found in the inner cytoplasmic leaflet, while the outer lipid monolayer is enriched in phosphatidylcholine, sphingomyelin, cholesterol and GSLs. ${ }^{14-16}$ Findings during the last 50 years (reviewed by TODESCHINI and HAKOMORI ${ }^{17}$ ) indicated that the structure of the extracellular leaflet is composed of spatially organized microdomains enriched in cholesterol, sphingolipids and associated proteins varying in structure and function. ${ }^{18}$

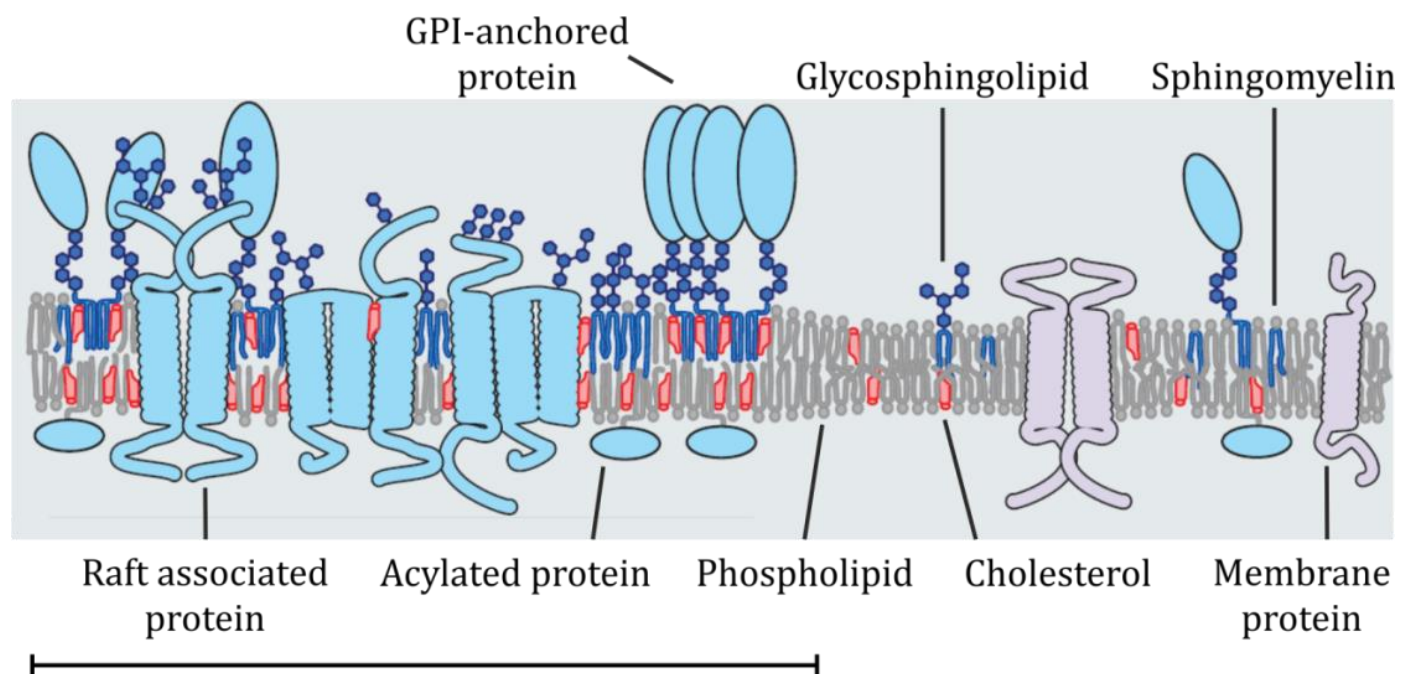

Raft "phase"

Figure 1.3: Schematic representation of the assumed lipid rafts in the extracellular plasma membrane ${ }^{\mathbf{1 9}}$, enriched in sphingolipids (glycosphingolipids and sphingomyelin, dark blue) and cholesterol (red), incorporating raft associated, GPI (glycosylphosphatidylinositol)-anchored and acylated proteins (light blue). The composition of the raft "phase" can be distinguished from its surroundings consisting of a higher amount of phospholipids and other membrane proteins.

The first steps in postulating the formation of microdomains were taken when GSL clustering was observed in various cell membranes, the Golgi apparatus and liposomes even without cholesterol. ${ }^{20-25}$ Another key step was the recognition of cholesterol- and sphingolipid-enriched detergent-resistant membranes (DEM), leading to the concept of GSL-enriched microdomains (GEM) ${ }^{26,27}$ and the postulation of the raft theory proposed by Simons and IKONEN ${ }^{28}$ in 1997 (cf. Figure 1.3). The approximately 20-200 $\mathrm{nm}^{29}$ wide lipid microdomains are associated with important cellular processes such as signal transduction ${ }^{30}$. 
In 2002, HAKOMORI ${ }^{31}$ added the term glycosynapse to the raft concept, which describes the glycosylation-dependent cell adhesion linked to signaling process. More specifically, membrane incorporated GSLs self-assemble to GSL-enriched microdomains (GEM) bearing diverse signal transducer molecules like cSrc or FAK. ${ }^{32,33}$

However, morphological evidence for those microdomains were mainly reported in model or sorely in specific biological membranes ${ }^{34}$ and a direct transfer to the plasma membrane of living cells remained questionable.

\subsection{GLYCOSPHINGOLIPIDS}

As a basic unit of cellular membranes, membrane lipids fulfill a wide variety of lifesustaining functions such as storage or signaling molecules. ${ }^{1,35}$ According to VAN MEER and DE KROON ${ }^{36}$, the plasma membrane of mammalian cells are composed of approximately $65 \mathrm{~mol} \%$ glycerolipids, $10 \mathrm{~mol} \%$ sphingolipids and $25 \mathrm{~mol} \%$ sterols such as cholesterol among various membrane-bound proteins. Glycerolipids consist of a glycerol backbone that is attached to two hydrocarbon chains and a phosphorylated alcohol. ${ }^{1}$

Sphingolipids were first discovered by THUDICHUM in $1884 .{ }^{37}$ The common structural feature of sphingolipids is a ceramide unit acting as a starting material of many other lipid structures. An overview of common sphingolipid structures is shown in Figure 1.4. The lipid class of sphingolipids are further subdivided into sphingomyelin (SM) and glycospingolipids (GSLs), both important components of mammalian cells. ${ }^{38}$ In general, sphingolipids are built up from longer hydrocarbon chains, which share saturated or unsaturated configurations. Due to this structural feature, high van-derWaals forces can exist between the hydrocarbon chains, resulting in a denser packing within the lipid membrane and an accumulation in a gel $\left(\mathrm{l}_{\beta}^{\prime}\right)$ or solid-ordered $\left(S_{0}\right)$ phase, which in turn is fluidizied by additionally incorporated sterols (cf. Chapter 2.4). While the ceramide backbone in SM is esterified with a phosphoethanolamine or phosphocholine head group, GSLs consist of mono, di- or oligosaccharide-containing head groups carrying a neutral, anionic (acidic) or cationic (basic) charge. ${ }^{32}$ The monosaccharide glucosylceramide represents the simplest GSL, while 
oligosaccharides such as the disaccharide lactosylceramide (LacCer, cf. Chapters 1.6 and 2.2.1) or the ganglioside $\mathrm{GM}_{\mathrm{M} 3}$ (cf. Chapters 1.5 and 2.2.1) form more complex structures. For example, gangliosides are characterized by an additional sialic acid residue. ${ }^{39,40}$
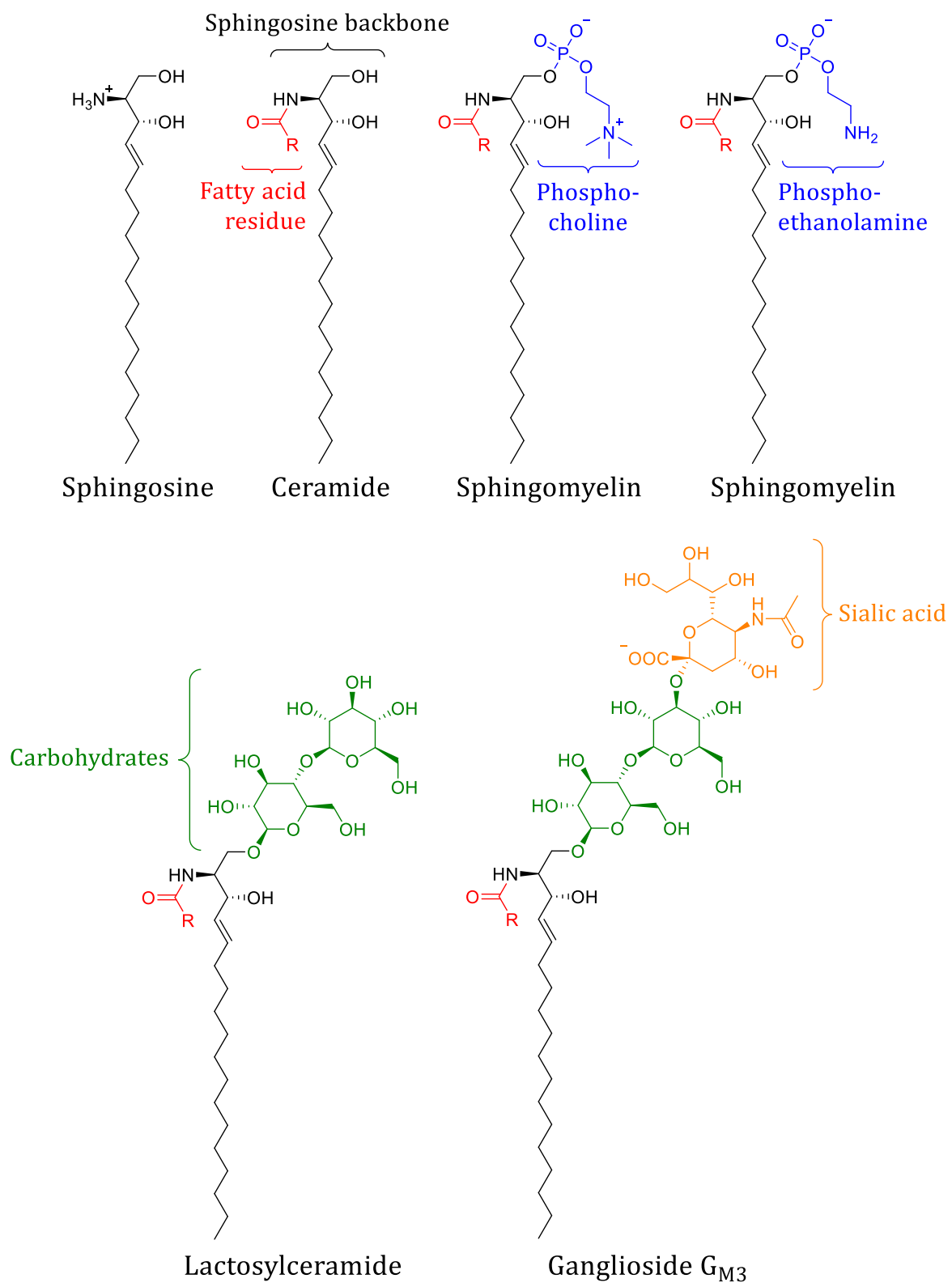

Figure 1.4: Overview of common sphingolipid structures characterized by a ceramide backbone, which consists of variable fatty acid residues (red). The ceramide backbone of sphingomyelin esterified with a phosphoethanolamine or phosphocholine head group (blue), while GLS like lactosylceramide or GM3 are composed of saccharide-containing head groups (green), with $\mathrm{G}_{\mathrm{M} 3}$ carrying an additional sialic acid residue (orange). $\mathrm{R}=$ alkyl residue of respective fatty acid. 


\subsubsection{CONFORMATION AND ORGANIZATION}

The presence of long hydrocarbon chains triggers the self-association of GSL into $S_{\mathrm{o}}$ phases (cf. Chapter 2.4). ${ }^{41}$ and may also couple the extra- and intracellular leaflet of plasma membranes by possible interdigitation processes. ${ }^{30}$

The ability of GSLs to form the so-called GSL-enriched microdomains (GEM) was first shown by x-ray crystallographic experiments of cerebrosides ${ }^{42}$ and minimum-energy models of various GSLs. ${ }^{43,44}$ In these experiments it was demonstrated that the axis of the carbohydrate head group of the entire GSL is perpendicular to the ceramide axis of the GSL. An exemplary minimum-energy model of the GSL Gb5 is shown in Figure 1.5. The oligosaccharide moiety is directed to the hydrophilic area outside of the cellular plasma membrane and offers a great variability of possible binding sites for complementary GSLs, toxins, lectins or antibodies ${ }^{31}$ (cf. Figure 1.5).

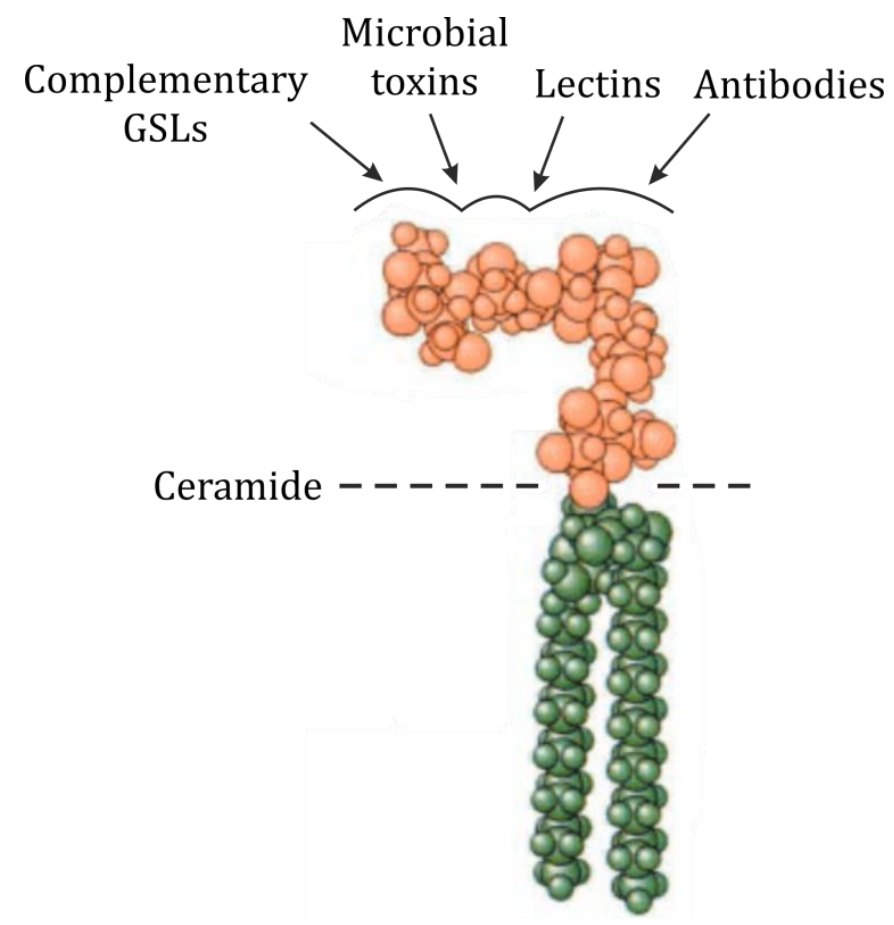

Figure 1.5: Minimum-energy model of the GSL Gb5 demonstrating the perpendicular orientation of the oligosaccharide head group (orange) to the ceramide axis (green) keeping the GSL in a defined orientation within the cellular plasma membrane. ${ }^{31}$ By a side-by-side interaction, GSLs form GSL-enriched microdomains. Due to the high variety of the oligosaccharide head group, GSLs are able to interact with a great amount of binding partners as complementary GSLs, toxins, lectins or antibodies.

On the other hand, the ceramide component is anchored within the membrane, characterizing the hydrophobic region. By this, the ceramide structure keeps the entire GSL in a defined conformation and orientation. A cis or side-by-side interaction between several GSLs within the plasma membrane leads to the formation of 
GEMs. ${ }^{32,45}$ Scanning electron microscopy studies pointed out that GEMs can be separated from glycerophospholipid or glycoprotein domains within the cell membrane 21,22 and that they have distinguishable properties compared to rafts or caveolae. ${ }^{32}$

Since some proteins associate with GEMs within the same plasma membrane, GSLs can modulate the activity of those proteins. Especially gangliosides are known to interact with receptor tyrosine kinases and therefore inhibit or promote growth factor receptors. ${ }^{46}$ Further, interactions of GEM with diverse signal transducer molecules like cSrc or FAK regulate signaling processes ${ }^{32,33}$ (cf. Figure 1.6). Such processes are possible due to the mentioned GSL interdigitations. Due to the long hydrocarbon chains, GSLs may protrude beyond the mid-plane of the bilayer and penetrate into the opposite leaflet. This could ensure the transmission of information from the outside to the inside of the cell. ${ }^{47}$

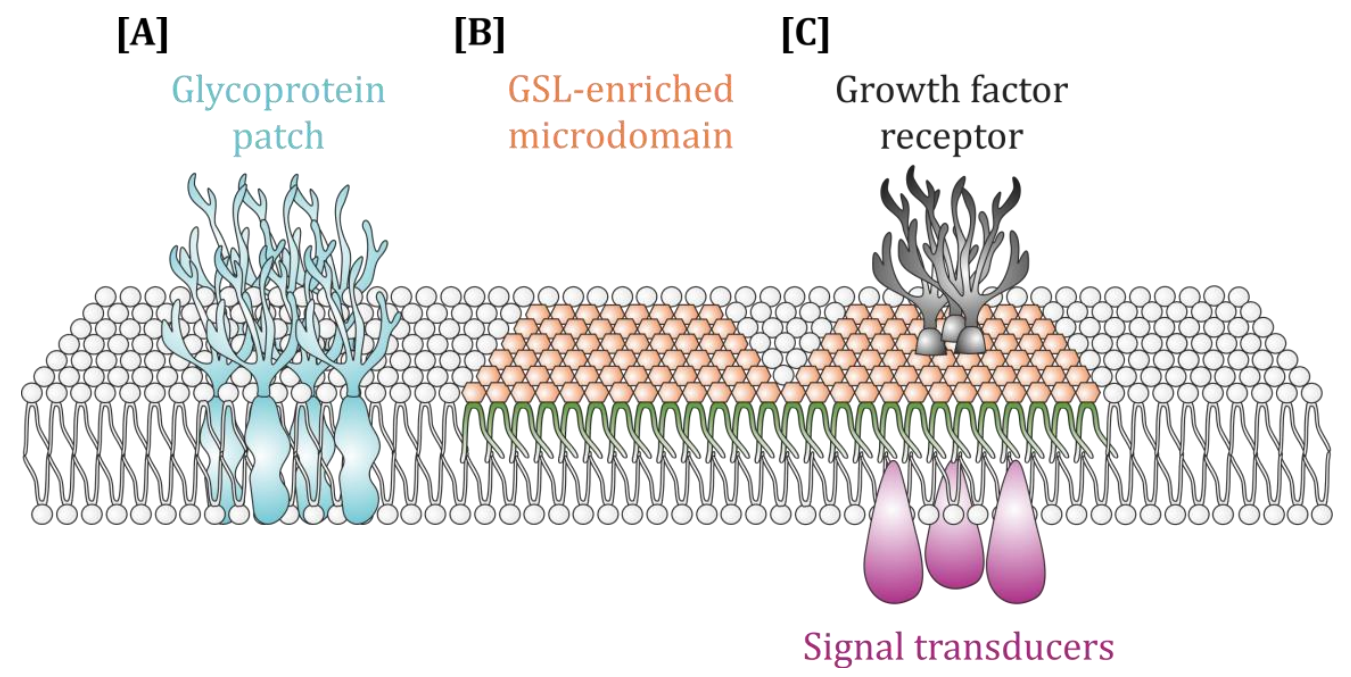

Figure 1.6: Schematic representation of GSL-enriched microdomains (GEMs, orange) within the extracellular leaflet of a plasma membrane. It is known that [A] GEMs can be separated from glycoprotein patches (blue) and shows [B] no or [C] stable associations with signal transducers (violet) or growth factor receptors (black).

The asymmetric distribution of membrane lipids within the extracellular and intracellular leaflets of the plasma membrane has already been demonstrated by several studies. ${ }^{48}$ Glycosylated proteins and lipids ("glycocalyx") in particular were predominantly found to be enriched within the extracellular leaflet with their carbohydrate head groups facing the surrounding medium of the cell. ${ }^{49}$ With regard to cellular recognition and adhesion processes, special focus was placed on the impact of protein-protein or carbohydrate-protein interactions, while carbohydratecarbohydrate interactions (CCIs) between GSLs on neighboring cells were considered 
to be too weak to mediate cell recognition and adhesion. ${ }^{45}$ However, due to their flexible chains and therefore high specificity, homotopic (Lewis ${ }^{\mathrm{X}}$-Lewis ${ }^{\mathrm{X}}{ }^{50,51}$ or heterotopic ( $\left.\mathrm{G}_{\mathrm{M} 3}-\mathrm{Gg} 3 / \mathrm{LacCer}\right)^{33,52}$ trans interactions of complementary GSLs have been found to fulfill the prerequisites to be able to mediate those short-termed events. Yet, only few studies addressed the impact on CCIs on cell-cell interactions. For invertebrates (marine sponges), carbohydrate-rich proteoglycans are found to participate in cell recognition, while in vertebrate models, CCIs seem to be responsible in mouse embryo compaction and mediate cancer cell adhesion. ${ }^{45}$

\subsection{CARBOHYDRATE-CARBOHYDRATE INTERACTIONS}

\subsubsection{MOLECULAR FORCES}

For a long time, carbohydrate-carbohydrate interactions (CCIs) were claimed to be too weak and nonspecific to play an important role in cellular recognition and adhesion processes relative to the widely accepted protein-protein (PPI) and carbohydrate-protein (CPI) interactions. However, due to their high variability and the accumulation of GSLs in GEMs, CCIs actually offer polyvalent intercellular forces ${ }^{53}$ of relatively high adhesion strengths in the range of several hundred $\mathrm{pN}$, which are comparable to adhesion forces obtained in PPIs and CPIs. ${ }^{45,54}$ The discovery of the crystal structure of the GSL Lewis ${ }^{\mathrm{X}}$ by PÉREz et al. ${ }^{55}$ revealed that GSLs offer the possibility to accept but also donate hydrogen bonds due to a great supply of hydroxyl, amine and carboxyl groups ${ }^{32}$ (cf. Figure 1.7, blue-colored dashed lines). Within the crystal structure, a great amount of hydrogen bonds were detected both intramolecular and intermolecular and occur likewise with the surrounding medium. 55

In general, CCIs are based on van-der-Waals forces, including dipole-dipole and London dispersion forces. ${ }^{56}$ Additionally, the binding strength of neighboring carbohydrate residues can be stabilized via complementary hydrophobic surfaces (cf. Figure 1.7, magenta-colored area) and calcium ions (cf. Figure 1.7, green-colored arrows) which can bridge the negative charge of acidic GSLs or rearrange the carbohydrate unit to allow proper interaction strength. ${ }^{56}$ In order to ensure a tight binding between GSLs incorporated into neighboring cellular plasma membranes, 
SPILLMANN ${ }^{57}$ proposed a zipper-like anchoring of the GSL chains within the opposing membranes. Based on this, it can be stated that GSLs offer flexible chains and variable binding sides to create flexible and versatile carbohydrate-carbohydrate recognition systems for cellular adhesion processes, which might be established before rather stable PPIs occur.

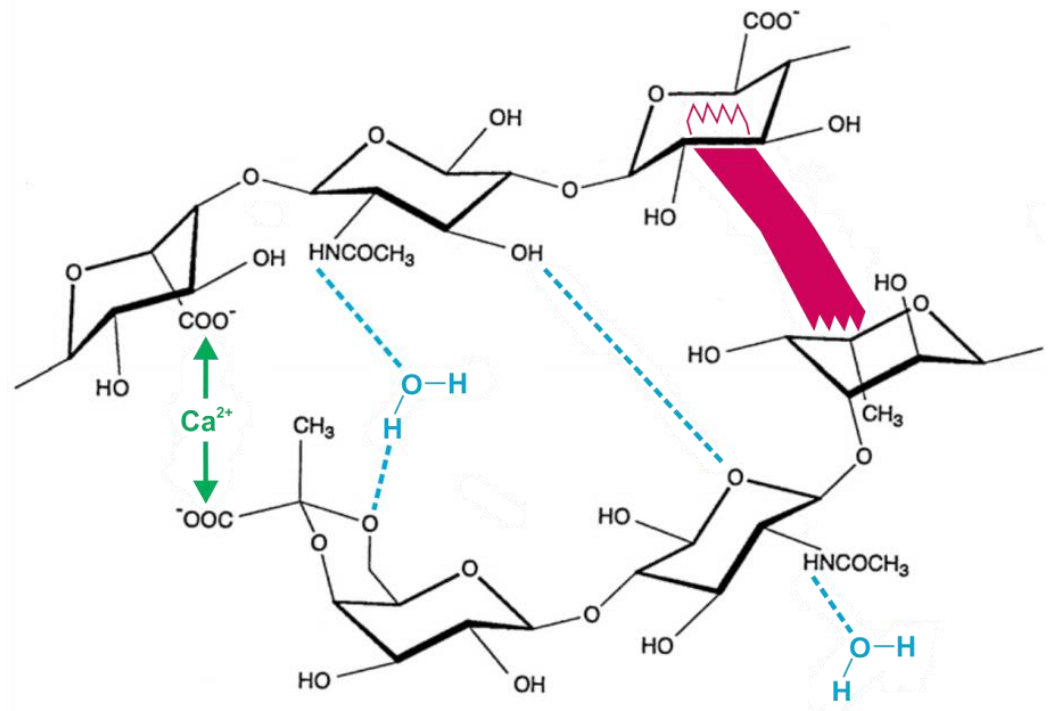

Figure 1.7: Schematic illustration of molecular forces between carbohydrate units in GSLs on opposing cellular plasma membranes. ${ }^{58}$ Carbohydrate-carbohydrate interactions can be stabilized due to hydrogen bonds (bluecolored dashed lines) between hydroxyl, amine and carboxyl groups, hydrophobic surfaces (magenta-colored shaded area) and the bridging effect of ionic interactions by $\mathrm{Ca}^{2+}$ ions (green-colored arrows).

\subsection{BiofunCtionAL ROLE OF $\mathrm{G}_{\mathrm{M} 3}$}

In vertebrates, Gм3 makes up the majority of gangliosides within the cell membranes of most extraneural tissues (reviewed by PROKAzova et al.59). It is the metabolic precursor for more complex gangliosides and therefore directly influences their contents in the cells ${ }^{59}$. Gangliosides are functionally associated with receptor and nonreceptor tyrosine kinases, cellular antigens, receptors and adhesion molecules in lipid GEMs, which are responsible for signal transduction, membrane transport or cell adhesion. ${ }^{59}$ Besides, $\mathrm{G}_{\mathrm{M} 3}$ is proposed to alter the molecular organization in glycosynaptic microdomains and to modulate the activation levels of co-localized signaling molecules involved in cancer pathogenesis thus influencing cell adhesion, growth and motility ${ }^{60}$. 
Several processes like cell proliferation and differentiation as well as apoptosis, embryogenesis and oncogenesis are regulated under involvement of $\mathrm{G}_{\mathrm{M} 3 .}{ }^{59}$

$\mathrm{G}_{\mathrm{M} 3}$ influences fibroblast growth factor (FGF) and platelet-derived growth factor (PDGF) by reducing tyrosine phosphorylation thus stimulating cell proliferation ${ }^{61}$. In addition, contact inhibition of locomotion and proliferation are absent in cancer cells. Studies observed an association of the loss of contact inhibition in cancer cells with an increase in LacCer and a twofold decrease in $\mathrm{GM}_{\mathrm{M}}$ content. ${ }^{59}$ The inhibitory effect of $\mathrm{G}_{\mathrm{M} 3}$ on the growth of tumor cells and tumor development is related to its property to suppress tyrosine phosphorylation of growth factor receptors (reviewed by PROKAZOVA et al. ${ }^{59}$ ). Findings of ZHENG et al. ${ }^{62}$ propose a role of $\mathrm{G}_{\mathrm{M} 3}$ in the function of integrin receptors, which are crucial for cell adhesion. During initial adhesion, integrin receptors promote interaction between the cytoskeleton and extracellular proteins (fibronectin and laminin) until the adhesion is terminated by the formation of focal adhesions mainly induced by FAK (focal adhesion kinase) causing signal transduction. $\mathrm{G}_{\mathrm{M} 3}$ can also inhibit invasive tumor cells by enhancing cell adhesion to the intercellular matrix by promoting the interaction between membrane proteins like CD9 with $\alpha 3$-integrin. ${ }^{59}$

The effect of $\mathrm{G}_{\mathrm{M} 3}$ on the proliferation depends on the ratio between $\mathrm{G}_{\mathrm{M} 3}$ and more complex gangliosides as proliferation can either be induced or suppressed.63,64 Additionally, АвАTE et al. ${ }^{65}$ showed that the influence of GM3 on tumor growth and angiogenesis depends also on the ratio between $\mathrm{G}_{\mathrm{M} 3}$ and complex gangliosides.

For Gм3-expressing mouse melanoma B16 cells it is established that an interaction between $\mathrm{G}_{\mathrm{M} 3}$ and LacCer is responsible for the observed adhesion. ${ }^{66}$ PROKAzovA et al. ${ }^{9}$ found in trials with four types of B16 cells varying in their $\mathrm{G}_{\mathrm{M} 3}$ expression levels that the measured adhesion was dependent on Gм3 expression. They proposed that the interaction between $\mathrm{G}_{\mathrm{M} 3}$ and LacCer is an initial adhesion process by cancer cells to anchor metastatic foci.

To conclude, it can be said that the specific role of $\mathrm{G}_{\mathrm{M} 3}$ regarding metastasis varies with its concentration on the tumor cell surface as well as with its concentration in the environmental surroundings. ${ }^{67}$ 


\subsection{BIOFUNCTIONAL ROLE OF LACCER}

LacCer is a key intermediate for higher glycosylated sphingolipids like the ganglioside Gм3. It is associated to cell-cell and cell-matrix interactions and to signaling processes concerning cell differentiation, development, apoptosis and oncogenesis ${ }^{68}$. LacCer is a part of the sphingolipid-sterol raft domains and has been identified as an essential component of rafts in kidney cortex microvillar membranes ${ }^{69}$ and in the membranes of human neutrophils ${ }^{70}$. Raft domains are associated with various lipid-anchored proteins and proposed to play an important role in transmembrane signaling processes. ${ }^{28,30,71,72}$ Especially in human neutrophils LacCer is of great interest. In mature cells it makes up more than $70 \%$ of total GSLs. ${ }^{73}$ Human neutrophils are the first defense against microorganisms and are involved in inflammatory reactions. ${ }^{74}$ Iwabuchi et al.75 showed that LacCer is involved in superoxide generation and migration of neutrophils by activating of a Src family kinase within rafts.

The binding of LacCer to a variety of different microorganisms that are linked to pathogenic behavior (reviewed by IWABUCHI et $a l^{76}$ ) proposes a key role in pathogenhost interactions. Further it has been observed that in rats a certain soluble cell wall component ( $\beta$-glucan) of Pneumocystis carinii triggers a LacCer dependent mechanism that leads to the release of macrophage inflammatory protein-2 (MIP-2). ${ }^{77}$ Another $\beta$-glucan polysaccharide from Candida albicans was observed to induce chemotaxis of neutrophils also by LacCer-enriched microdomains ${ }^{78}$. Further studies underlying the involvement of LacCer in inflammatory signaling is the observed upregulation of LacCer synthase in glial proliferation ${ }^{79}$. Besides, CD11b/CD18 integrin, which regulates several functions in neutrophils such as adhesion, migration, chemotaxis and phagocytosis, is known to utilize LacCer enriched lipid rafts for phagocytosis. ${ }^{80}$

These findings led to the assumption that LacCer-enriched microdomains act as pattern recognition receptors (PRRs) on neutrophils and are therefore a key in the defense against pathogens. ${ }^{76}$ Likewise these membrane microdomains are targeted by invading microorganism, which alter the signaling cascade in order to escape the immune defense and establish an infection. ${ }^{81}$ 


\section{MATERIALS AND METHODS}

This thesis comprises cell studies and model membrane assays to investigate the ultra-weak, but highly specific carbohydrate-carbohydrate interaction between the ganglioside $\mathrm{G}_{\mathrm{M} 3}$ and the globoside lactosylceramide. In this chapter, information about the materials, setups and experimental procedures used in this work are described.

\subsection{B16 CELL LINES}

As a model for the carbohydrate induced attachment of carcinoma cells the GM3overexpressing murine B16 melanoma tumor cell lines B16-F1 and B16-F10 are used. Both cell lines differ in the degree of their metastatic potential. The B16-F1 cell line shows a rather low tendency for metastasis, whereas the B16-F10 cell line is characterized by a high degree of metastasis. ${ }^{5-7}$

Both the B16-F1 (ATCC-No.: CRL-6323, passage 28) and B16-F10 (ATCC-No.: CRL6475, passage unknown) cell lines were purchased from the AMERICAN TYPE CULTURE Collection (ATCC, Manassas, USA). Originally, the cell lines were derived from a naturally grown tumor cell, which was discovered in the skin tissue behind the ear of the mouse species C57BL/6 in 1954.

In Table 2.1, media and buffer solutions, which are used for general cell culture experiments as well as for staining and functionalization assays are listed. 
Table 2.1: Overview of the composition and ingredients of media and buffer systems used for cell experiments.

\section{Media / buffer system}

BSA

Bovine serum albumin

FBS

Fetal bovine serum

\section{HEPES}

\section{L-glutamine}

PBS--

Phosphate buffered saline without $\mathrm{Mg}^{2+} / \mathrm{Ca}^{2+}$

\section{Penicillin/streptomycin \\ Triton X-100 \\ DMEM 1X}

Dulbecco's modified eagle medium $1 \mathrm{X}$

$+4.5 \mathrm{~g} / \mathrm{L}$ glucose with L-glutamine

\section{D10F $^{-}$}

DMEM $1 \mathrm{X}+10 \%$ FBS

\section{D10F $^{+}$}

$\mathrm{D}_{10 \mathrm{~F}^{-}}+15 \mathrm{mM}$ HEPES $+200 \mu \mathrm{g} / \mathrm{mL}$ penicillin/streptomycin

\section{Blocking buffer}

$5 \%$ BSA $(w / v)$ in PBS--

\section{Dilution buffer}

$1 \% \operatorname{BSA}(w / v)$ in PBS--

\section{Triton blocking buffer}

$5 \%$ BSA $(w / v)+0.3 \%$ Triton-X-100 $(v / v)$ in PBS--

\section{Triton dilution buffer}

$1 \%$ BSA $(w / v)+0.3 \%$ Triton-X-100 $(v / v)$ in PBS--

\section{Manufacturer information}

Sigma AlDRICH, St. Louis, MO, USA

LifE Technologies, Carlsbad, CA, USA

Biochrom, Berlin, Germany

LoNZA, Basel, Switzerland

Biochrom, Berlin, Germany

GE HEALTHCARE, Logan, Utah

Sigma ALDRICH, St. Louis, MO, USA

LoNZA, Basel, Switzerland 


\subsubsection{CELl CULtuRE CONDITIONS}

The cell lines used in this work were thankfully cultivated and prepared by ANJA HERDLITSCHKE (former: Institute of Organic and Biomolecular Chemistry, GeorgAugust University, Göttingen, Germany), Angela RüBELING and DR. TABEA Oswald (Institute of Organic and Biomolecular Chemistry, Georg-August University, Göttingen, Germany).

In order to avoid contamination, cell culture and preparation were performed under sterile working conditions. For this purpose, a sterile Safe 2020 laminar flow (THERMo Fisher SCIENTIFIC, Waltham, MA, USA) was used with sterile equipment and sterile filtered solutions and media, which were heated up to $37^{\circ} \mathrm{C}$. In the following sections, solutions and media heated to $37^{\circ} \mathrm{C}$ are described as "warm". The cells were cultured in a humidified Heracell 160i incubator (THERmo FiSHER SCIEnTIFIC, Waltham, MA, USA) at $37{ }^{\circ} \mathrm{C}$ and $7.5 \% \mathrm{CO}_{2}$ in $25 \mathrm{~cm}^{2}$ or $75 \mathrm{~cm}^{2}$ cell culture flasks (TPP TEChNo Plastic Products AG, Trasadingen, Switzerland). The B16 cell lines were cultivated in a suitable D10F- medium (cf. Table 2.1).

\section{Cell tRYPSinization PROTOCOL}

Shortly before reaching the confluent phase after approximately $24 \mathrm{~h}$ to $48 \mathrm{~h}$ in the culture flasks, the cells were harvested, re-seeded or prepared for further studies. In order to harvest the cells, the old cell medium was removed and the cells were incubated for about $1 \mathrm{~min}$ with $2 \mathrm{~mL}$ or $3 \mathrm{~mL}$ of a warm trypsin/EDTA $(0.05 \% / 0.02 \%$, Biоснвом GмвH, Berlin, Germany) solution in the incubator, depending on the cell culture flask size. After the cells had detached from the bottom of the flask, they were transferred to a freshly prepared warm stop solution consisting

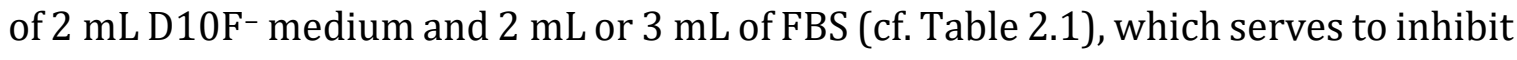
trypsin. The cell suspension was centrifuged with a Heraeus Megafuge 16R (Thermo Fisher SCIENTIFIC, Waltham, MA, USA) at $1000 \mathrm{rpm}(188 \mathrm{xg})$ for $4 \mathrm{~min}$. The supernatant was discarded and the obtained cell pellet resuspended in $1 \mathrm{~mL}$ of the desired medium or solution. The cell density was determined using a disposable CChip counting chamber (Bıоснвом GмвH, Berlin, Germany). For re-seeding, the pellet was resuspended in $1 \mathrm{ml}$ of a warm $\mathrm{D} 10 \mathrm{~F}^{-}$medium and transferred to cell culture flasks for $24 \mathrm{~h}$ to $48 \mathrm{~h}$, allowing the cells to grow to sub-confluency or confluency. 


\subsubsection{TAINING AND FUNCTIONALIZATION}

To study the organization and distribution of $\mathrm{G}_{\mathrm{M} 3}$ of the $\mathrm{B} 16$ cell line, various staining and functionalization assays were performed. In order to stain certain components of the cell, such as the cell nucleus or $\mathrm{G}_{\mathrm{M} 3}$ on the outer leaflet of the cell membrane, standardized immunostaining protocols were used. To determine the contribution of the interaction between $\mathrm{G}_{\mathrm{M} 3}$ and its binding partner lyso-lactosylceramide to singlecell adhesion forces, the cells were additionally functionalized for control experiments. This was achieved by blocking the binding site of $\mathrm{G}_{\mathrm{M} 3}$ with an anti- $\mathrm{G}_{\mathrm{M} 3}$ monoclonal IgM antibody or by inactivating it using the enzyme sialidase, which cleaves off the sialic acid in the $\mathrm{G}_{\mathrm{M} 3}$ molecule (for both cf. chapter 2.1.2.2).

\subsubsection{IMMUNOSTAINING PROTOCOLS}

\section{Cell fiXATION AND BLOCKING PROTOCOL}

The cells were harvested using the trypsinization procedure described in chapter 2.1.1 and resuspended in warm D10F- medium (cf. Table 2.1). After counting, approximately 200,000 cells were transferred to $1 \mathrm{~mL}$ of warm D10F- medium and grown to sub-confluency for $24 \mathrm{~h}$ in culture treated and sterilized $35 \mathrm{~mm}$ low $\mu$-dishes (IBIDI GMBH, Martinsried, Germany) or sterilized $35 \mathrm{~mm}$ glass bottom petri dishes with a 14 mm glass microwell (No. 1.5, MatTé Corporation, Ashland, MA, USA). For the immunostaining procedure, the $\mathrm{D} 10 \mathrm{~F}^{-}$medium was removed and the cell sample was washed three times with $1 \mathrm{~mL}$ of a warm PBS-- solution (cf. Table 2.1). Then, the cells were fixed by incubating the cell layer with $1 \mathrm{~mL}$ of a paraformaldehyde (PFA, $4 \%$ in PBS--, FLUKA, Switzerland) solution for $20 \mathrm{~min}$ at room temperature. Again, the sample was washed three times with $1 \mathrm{~mL}$ of a PBS-- solution and treated with $1 \mathrm{~mL}$ of tritoncontaining or triton-free blocking buffer (cf. Table 2.1) for $30 \mathrm{~min}$ at room temperature to block unspecific binding of the antibodies. Another three-wash step with $1 \mathrm{ml}$ of PBS-- was carried out to prepare the cells for the staining procedure explained in the following sections.

\section{GM3 STAINING PROTOCOL}

In order to stain the glycosphingolipid (GSL) GM3 on the B16-F1 and B16-F10 cells' membranes, the sub-confluent grown cell layer was treated with the cell fixation and 
blocking protocol. For this purpose, a triton-free blocking buffer was used (cf. Table 2.1). The PBS-- solution was removed and the cells were incubated with $195 \mu \mathrm{L}$ of a $10 \mu \mathrm{g} / \mathrm{mL}$ solution of an anti-GM3 monoclonal IgM antibody (TCI DEUTSCHLAND GMBH, Eschborn, Germany), dissolved in a triton-free blocking buffer solution (cf. Table 2.1). After $1 \mathrm{~h}$ incubation at room temperature, the cells were washed with $1 \mathrm{~mL}$ PBS- on a shaker (80 rpm, GFL, Burgwedel, Germany) for $5 \mathrm{~min}$. After that, the cells were incubated with $5 \mu \mathrm{g} / \mathrm{mL}$ of a goat anti-mouse IgG/IgM secondary antibody conjugated with Alexa Fluor 488 (Life Technologies, Carlsbad, Germany), diluted in triton-free dilution buffer (cf. Table 2.1), for $1 \mathrm{~h}$ at room temperature. Again, the sample was washed three times with $1 \mathrm{~mL}$ PBS-- by shaking the sample for five minutes between the washing steps. The cells were directly used or stored at $4{ }^{\circ} \mathrm{C}$ for a maximum of $24 \mathrm{~h}$.

\section{Cell NUCleus StAining PROTOCOL}

Two different fluorescence markers were used in order to examine the cell nucleus of the B16-F1 and B16-F10 cells.

For confocal laser scanning microscopy studies, 4',6-diamidino-2-phenylindole (DAPI, Life TEChNologies, Carlsbad, Germany) was used. DAPI shows the preference to accumulates in AT-rich regions of the double-stranded deoxyribonucleic acid (DNA) in the nucleus.82 DAPI-staining of the nucleus was combined with the visualization of $\mathrm{G}_{\mathrm{M} 3}$. First, the sub-confluent cell layers were treated based on the $\mathrm{G}_{\mathrm{M} 3}$ staining protocol. Subsequently, the PBS-- was removed and $50 \mathrm{ng} / \mathrm{mL}$ of DAPI in a triton-containing dilution buffer (cf. Table 2.1) was pipetted onto the cells. After an incubation time of $15 \mathrm{~min}$ at room temperature, the cells were washed three times with $1 \mathrm{~mL}$ of a PBS - solution and used directly or stored at $4{ }^{\circ} \mathrm{C}$ for a maximum of $24 \mathrm{~h}$.

For cell migration studies, the fluorescence marker Hoechst 33342 (Trihydrochloride, Trihydrate, THERMo FISHER SCIENTIFIC, Waltham, MA, USA) was used. Just like DAPI, the bisbenzimide accumulates in AT-rich regions of the double-stranded DNA. ${ }^{83}$ Due to its ability to be cell permeable, Hoechst 33342 is a widely used dye for live cell experiments. ${ }^{84}$ Therefore, B16-F1 and B16F10 cells were treated according to the trypsinization protocol (cf. chapter 2.1.1) and counted in a warm D10F- medium (cf. Table 2.1). Afterwards, $1 \mu \mathrm{g} / \mathrm{mL}$ of a Hoechst 33342 solution in $\mathrm{D} 10 \mathrm{~F}^{+}$medium (cf. 
Table 2.1) was used to stain 20,000 live cells in solution. The cell solution was directly used to perform cell migration experiments. More information on the experimental procedure of the adhesion assay can be found in Chapter 2.8.6.

\subsubsection{FUNCTIONALIZATION PROTOCOLS}

\section{BLOCKING $\mathrm{G}_{\mathrm{M} 3}$ WITH ANTI-GM3 MONOCLONAL IGM ANTIBODY}

In order to block the binding site of the glycosphingolipid $\mathrm{GM}_{\mathrm{M} 3}$, which is overexpressed at the surface of the murine B16-F1 and F10 cell lines ${ }^{85,86}$, an anti-GM3 monoclonal IgM antibody was used. By this, we attempt to inhibit the interaction between Gмз and Lyso-LacCer.

First, the cells were detached from the bottom of the cell culture bottle as described in Chapter 2.1.1. Subsequently, the cells were taken up in $1 \mathrm{~mL}$ of a warm PBS-solution and counted. Approximately 500,000 cells were transferred to $500 \mu \mathrm{L}$ of a warm PBS-- solution and centrifuged for $3 \mathrm{~min}(0.3 \mathrm{xg})$ at $4{ }^{\circ} \mathrm{C}$ in a micro test tube (1.5 mL, Eppendorf, Hamburg, Germany). The supernatant was discarded and the resulting pellet was carefully resuspended in $500 \mu \mathrm{L}$ of a blocking buffer $5 \%$ BSA in

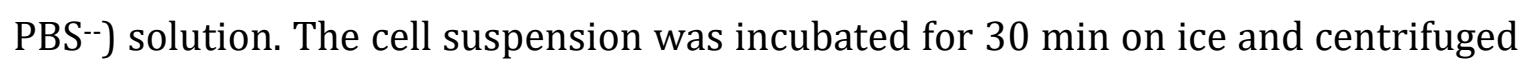
$\left(0.3 \times \mathrm{g}, 3 \mathrm{~min}, 4^{\circ} \mathrm{C}\right)$. Subsequently, the pellet was resuspended in $500 \mu \mathrm{L}$ of a PBS-solution and centrifuged $\left(0.3 \times \mathrm{g}, 3 \mathrm{~min}, 4^{\circ} \mathrm{C}\right)$. Again, the supernatant was discarded and the pellet carefully resuspended in $100 \mu \mathrm{L}$ of a $10 \mu \mathrm{g} / \mathrm{mL}$ anti-GM3 monoclonal IgM antibody solution. After an incubation period of about $1 \mathrm{~h}$ at $4{ }^{\circ} \mathrm{C}$, the solution was washed three times with a PBS-- solution and finally resuspended in $500 \mu \mathrm{L}$ of a warm D10F ${ }^{+}$medium. Single-cell force spectroscopy (SCFS) experiments (cf. chapter 2.6.1) were performed within 30 minutes after the functionalization in order to obtain single cells, which were well suited for an attachment to the cantilever.

\section{Cell functionalization With Sialidase}

In addition to antibody blocking, the enzyme neuraminidase from vibrio cholerae (sialidase, MERCK, Munich, Germany) was used to prevent the interaction between $\mathrm{G}_{\mathrm{M} 3}$ on the cells and lyso-LacCer. The enzyme is known to hydrolyze $\alpha 2,3-, \alpha 2,6$ - or $\alpha 2,8$ glycosidic linkages of terminal sialic residues in oligosaccharides, glycoproteins or glycolipids. 
First, the cells were detached from the bottom of the cell culture bottle as described in Chapter 2.1.1. Afterwards, the cells were taken up in $1 \mathrm{~mL}$ of a warm PBS-- solution and were counted. Approximately 500,000 cells were transferred to $50 \mu \mathrm{l}$ of $1 \mathrm{U} / \mathrm{mL}$ active sialidase, diluted to $500 \mu \mathrm{L}$ with warm PBS--. The cell suspension was incubated for $30 \mathrm{~min}$ on ice and centrifuged $\left(0.3 \mathrm{xg}, 3 \mathrm{~min}, 4^{\circ} \mathrm{C}\right)$. For the subsequent washing step, the pellet was resuspended in $500 \mu \mathrm{L}$ of a PBS-- solution and centrifuged $0.3 \mathrm{x} g$, $3 \mathrm{~min}, 4^{\circ} \mathrm{C}$ ). Again, the supernatant was discarded and the pellet was carefully resuspended in $500 \mu \mathrm{L}$ of a warm $\mathrm{D} 10 \mathrm{~F}^{+}$medium. Again, the cells were used within 30 min for SCFS (cf. chapter 2.6.1).

\subsection{LIPIDCHEMICAL EXPERIMENTAL PROCEDURES}

\subsubsection{LIPIDS}

The application of artificial lipid membranes including solid supported lipid membranes (SSLMs) is a widespread method to mimic the plasma membrane of many components in living organisms. ${ }^{10}$ Within this thesis, the glycerophospholipid POPC, (cf. Figure 2.1) serves as a matrix lipid, while the GSLs lyso-LacCer (cf. Figure 2.3) and $\mathrm{G}_{\mathrm{M} 3}$ (cf. Figure 2.4) function as receptor lipids for the interaction between their carbohydrate head groups. To visualize the SSLMs, the lipid-bound dyes $\beta$-Bodipy ${ }^{\mathrm{TM}}$ and Texas RedTM DHPE were used.

\section{1-PALMITOYL-2-OLEOYL-SN-GLYCERO-3-PHOSPHOCHOLINE}

1-Palmitoyl-2-oleoyl-sn-glycero-3-phosphocholine (POPC, SigMA-ALDRICH ${ }^{\circledR}$, St. Louis, MO, USA, cf. Figure 2.1) is an asymmetric synthetic glycerophospholipid that consists of a glycerol backbone, two nonpolar fatty acid side chains and a zwitterionic polar phosphocholine head group. The linear carbon side chains are composed of a saturated palmitic acid (C16:0) and an unsaturated oleic acid (C18:1) moiety having a cis (Z)-configured double bond at position $\Delta^{9}$. The latter lowers the main phase transition temperature to around $T_{\mathrm{M}}=-2{ }^{\circ} \mathrm{C}^{87-89}$, leaving POPC molecules in a fluid liquid-disordered $\left(l_{\mathrm{d}}\right)$ phase at room temperature. 


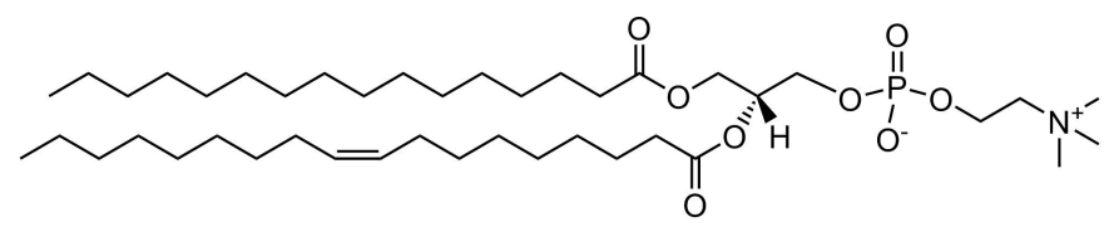

Figure 2.1: Chemical structure of 1-Palmitoyl-2-oleoyl-sn-glycero-3-phosphocholine, $\mathrm{C}_{42} \mathrm{H}_{82} \mathrm{NO}_{8} \mathrm{P}, M_{\text {POPC }}=760.08 \mathrm{~g} / \mathrm{mol} .{ }^{90}$

\section{LACTOSYLCERAMIDE DERIVATIVES}

The GSL lactosylceramide is expressed at the surface of human neutrophils ${ }^{70}$ and plays a significant role in a wide range of biological processes (cf. Chapter 1.6). Within this thesis, the synthetically made D-Lactosyl- $\beta-1,1^{\prime}-N$-palmitoyl-D-erythrosphingosine (LacCer, d18:1/16:0-lactosylceramide, AvANTI PoLAR LIPIDS INC., Alabaster, AL, USA, cf. Figure 2.2) and D-Lactosyl-ß-1-1'-D-erythro-sphingosine (lysoLacCer, d18:1-Lactosylceramide, SigmA-ALDRICH ${ }^{\circledR}$, St. Louis, MO, USA, cf. Figure 2.3) were used as receptor lipids for the ganglioside $\mathrm{G}_{\mathrm{M}}$.

LacCer consists of a hydrophobic ceramide backbone that is composed of a sphingosine (d18:1) unit having a trans (E)-configured double bond at position $\Delta^{4}$. The sphingosine in turn is $N$-linked to a saturated palmitic acid (C16:0) and holds a lactose residue via a glycosidic bond at its first position. Due to the high symmetry, the additional trans-configuration and almost identical length, strong van-der-Waals forces act between the chains leading to a drastic increase in the main phase transition temperature of around $T_{\mathrm{M}}=80^{\circ} \mathrm{C}^{91}$ in comparison to glycerophospholipids like POPC (cf. Figure 2.1). Thus, the entire glycosphingolipid remains in a solid-like $\left(S_{0}\right)$ phase under physiological conditions.

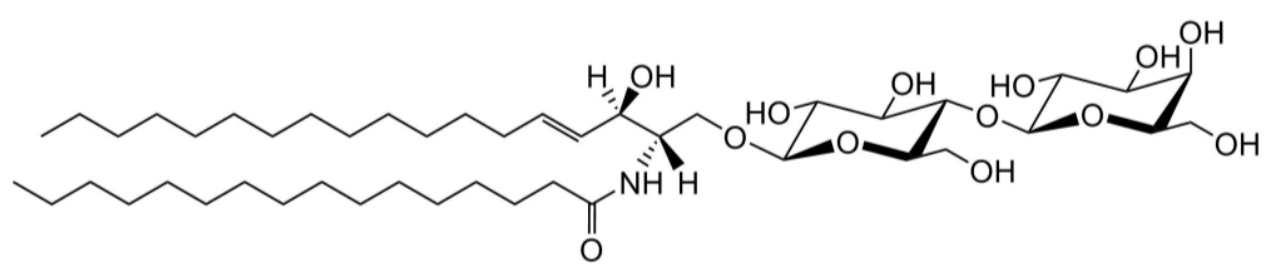

Figure 2.2: Chemical structure of D-Lactosyl- $ß-1,1^{\prime}-N$-palmitoyl-D-erythro-sphingosine, $\mathrm{C}_{46} \mathrm{H}_{87} \mathrm{NO}_{13}, M_{\text {LacCer }}=862.18 \mathrm{~g} / \mathrm{mol}^{.92}$

Lyso-LacCer, the $N$-deacylated derivative of LacCer, is also characterized by the disaccharide lactose as its hydrophilic head group, which is linked to a sphingosine unit via a glycosidic bond. Contrary to LacCer, the sphingosine is not bound to an additional fatty acid chain. Therefore, lyso-LacCer is anchored via the single-chain 
sphingosine component in the lipid membrane. The resulting asymmetry and the lack of stabilizing forces result in a lower main phase transition temperature of around $T_{\mathrm{M}}=40-50^{\circ} \mathrm{C}$ (cf. Chapter 3.3). Accordingly, lyso-LacCer remains in a solid-like phase under physiological conditions. Due to the reduced main phase transition temperature lyso-LacCer is the preferred lipid within this thesis in order to analyze the carbohydrate-carbohydrate interaction with the ganglioside $\mathrm{G}_{\mathrm{M} 3}$.

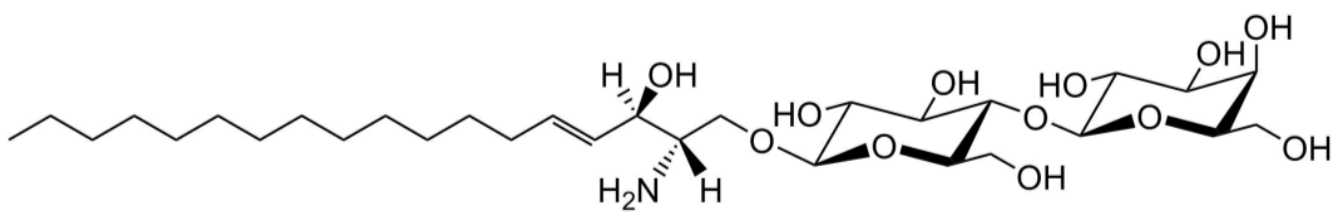

Figure 2.3: Chemical structure of D-Lactosyl- $\beta-1-1$ '-D-erythro-sphingosine, $\mathrm{C}_{30} \mathrm{H}_{57} \mathrm{NO}_{12}, M_{\text {lyso-LacCer }}=623.77 \mathrm{~g} / \mathrm{mol} .{ }^{93}$

\section{GANGLIOSIDES}

The GSL Gm3 (bovine milk, Avanti Polar LiPIDS Inc., Alabaster, AL, USA, cf. Figure 2.4) is a part of the ganglioside family and is, like LacCer, of immense importance in many key cellular processes, especially within the nervous system (cf. Chapter 1.5]. In this thesis, $\mathrm{G}_{\mathrm{M} 3}$ functions as a binding partner for LacCer or lyso-LacCer within the cell and model membrane studies.

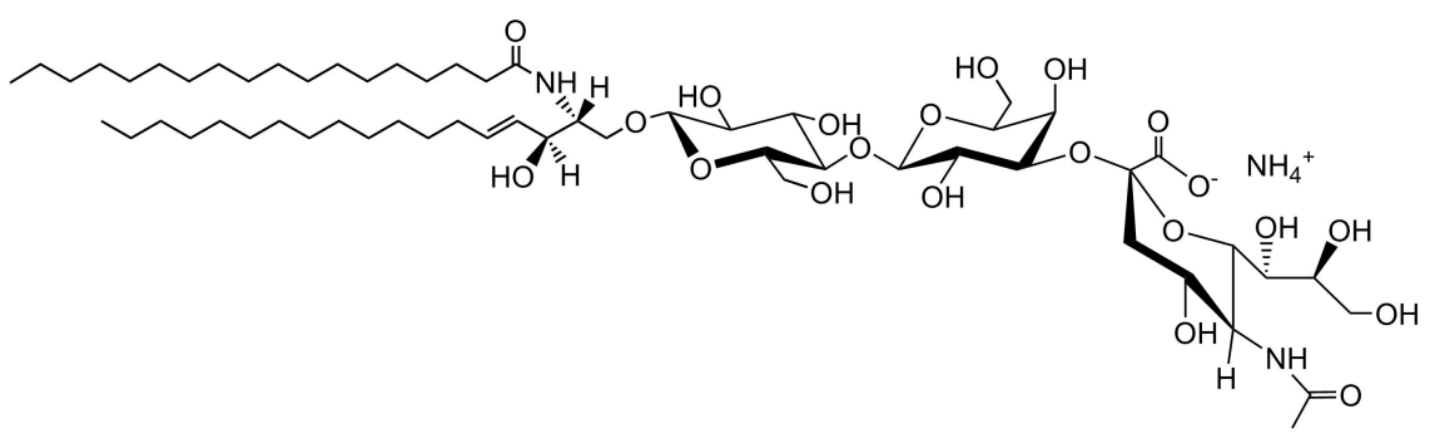

Figure 2.4: Representative chemical structure of $\mathrm{G}_{\mathrm{M} 3}, \mathrm{C}_{46} \mathrm{H}_{87} \mathrm{NO}_{13}, M_{\mathrm{GM}}=1267.85 \mathrm{~g} / \mathrm{mol} .{ }^{94}$

As all GSLs, Gм3 is characterized by a hydrophobic ceramide backbone consisting of a sphingosine (d18:1) unit having a trans (E)-configured double bond at position $\Delta^{4}$. The sphingosine is $N$-linked to a saturated stearic acid (C18:0) chain at its first position and holds an oligosaccharide head structure containing a sialic acid unit via a glycosidic bond. Various studies observed a main phase transition temperature of around $T_{\mathrm{M}}=35^{\circ} \mathrm{C}^{95-97}$. 


\subsubsection{FLUORESCENT PROBES}

\section{$\beta$-BODIPY ${ }^{\mathrm{TM}} \mathrm{C}_{12}$-HPC}

The lipid-coupled dye 2-(4,4-Difluoro-5-methyl-4-bora-3a,4a-diaza-s-indacen-3dodecanoyl)-1-hexadodecanoyl-sn-glycero-3-phosphocholine ( $\beta$-Bodipy ${ }^{\mathrm{TM}} \mathrm{C}_{12}$-HPC, $\beta$-Bodipy ${ }^{\text {TM }}$, Thermo FiSher SCIEnTIFIC, Waltham, MA, USA, cf. Figure 2.5) is a frequently used fluorophore in lipid membrane research due to its high extinction coefficient, quantum yield and photostability. ${ }^{98} \beta$-Bodipy ${ }^{\mathrm{TM}}$ has the same structural backbone as the matrix lipid POPC with a polar phosphocholine headgroup and a saturated palmitic acid (C16:0) chain linked to position 1 of the glycerol backbone. Position 2 on the contrary is esterified with a saturated lauric acid (C12:0) chain that is additionally linked to a boron-dipyrromethene (Bodipy ${ }^{\mathrm{TM}}$ ) fluorophore unit. Although the linear carbon side chains are anchored deep into the membrane due to the high hydrophobicity ${ }^{34}$, the bulky Bodipy ${ }^{\mathrm{TM}}$ unit prevents the phospholipid from forming an orderly membrane structure and therefore accumulates in the fluid liquid-disordered $\left(l_{\mathrm{d}}\right)$ phase. The emission maximum of $\beta$-Bodipy ${ }^{\mathrm{TM}}$ is $\lambda_{\mathrm{em}}=510 \mathrm{~nm}$ and the absorption maximum $\lambda_{\mathrm{ex}}=500 \mathrm{~nm}$.

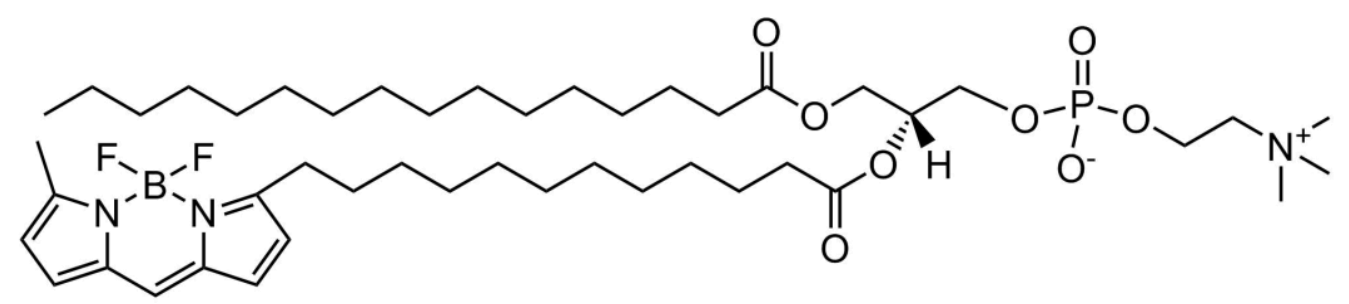

Figure 2.5: Chemical structure of 2-(4,4-Difluoro-5-methyl-4-bora-3a,4a-diaza-s-indacen-3-dodecano-yl)-1hexadodecanoyl-sn-glycero-3-phosphocholine, $\mathrm{C}_{46} \mathrm{H}_{79} \mathrm{BF}_{2} \mathrm{~N}_{3} \mathrm{O}_{8} \mathrm{P}, M_{\beta}$-Bodipy $=881.93 \mathrm{~g} / \mathrm{mol} .{ }^{99}$

\section{TEXAS RED ${ }^{\mathrm{TM}}$ DHPE}

With its similar stable fluorescence and high quantum yield ${ }^{100}$ compared to $\beta$ Bodipy $^{\mathrm{TM}}$ (cf. Figure 2.5), the lipid-coupled dye Texas Red ${ }^{\mathrm{TM}}$ 1,2-dihexadecanoyl-snglycero-3-phosphoethanolamine (Texas Red ${ }^{\text {TM }}$ DHPE, THERMo FiSHER SCIENTIFIC, Waltham, MA, USA, cf. Figure 2.6) is well suited to visualize and analyze membrane properties.

Texas Red ${ }^{\mathrm{TM}}$ DHPE is a synthetically produced phospholipid that is composed of a polar phosphoethanolamine headgroup and two saturated palmitic acids (C16:0) chains connected to position 1 and 2 of the glycerol backbone. The fluorescence 
activity of the lipid is obtained by the polycyclic sulforhodamine 101 acid chloride (Texas Red ${ }^{\mathrm{TM}}$ ) that is covalently bound to the headgroup. By replacing the typical phosphocholine headgroup with phosphoethanolamine, the overall fluorescent lipid carries a net charge of -1 e. ${ }^{101}$ Computational studies by Skaug et al. ${ }^{101,102}$ showed that the polycyclic dye Texas Red ${ }^{\mathrm{TM}}$ is located at the interface of a lipid membrane rather than sticking out to the hydrophilic water phase. These findings and also the size of the dye lead to an accumulation of Texas Red ${ }^{\text {TM }}$ DHPE in fluid liquid-disordered ( $l_{\mathrm{d}}$ ) phase. The emission maximum is $\lambda_{\mathrm{em}}=607 \mathrm{~nm}$ and the absorption maximum $\lambda_{\mathrm{ex}}=584 \mathrm{~nm}$.

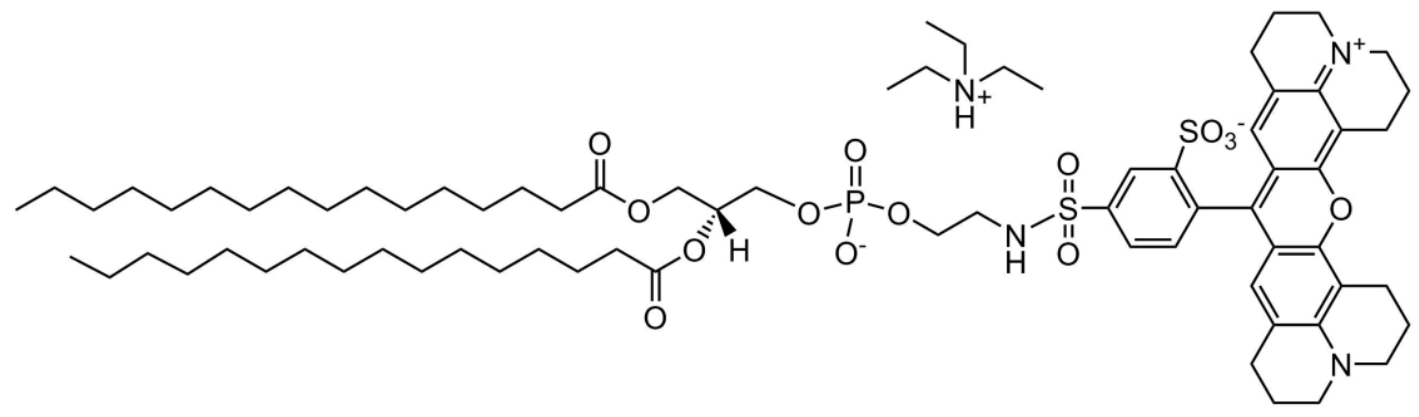

Figure 2.6: Chemical structure of Texas Red 1,2-Dihexadecanoyl-sn-glycero-3-phosphoethanolamin, $\mathrm{C}_{74} \mathrm{H}_{117} \mathrm{~N}_{4} \mathrm{O}_{14} \mathrm{PS}_{2}, M_{\text {Texas Red DHPE }}=1381.85 \mathrm{~g} / \mathrm{mol} .103$

\subsubsection{PREPARATION OF SMALL UNILAMELLAR VESICLES}

In this thesis, small unilamellar vesicles (SUVs) are used to produce SSLMs by spreading vesicles composed of the desired lipid mixture on glass or silicon dioxide $\left(\mathrm{SiO}_{2}\right)$ substrates above the highest main phase transition temperature of the used lipids. The attachment and fusion of the vesicles on the chosen substrate result in a homogenous coverage of the surface with a lipid membrane.

\subsubsection{EXPERIMENTAL PROCEDURE}

The buffer systems and lipid mixtures used for SSLMs are listed in Table 2.2 and Table 2.3 , respectively. The buffers were prepared with ultra-pure water, filtered (cellulose acetate filter, $0.2 \mu \mathrm{m}$ pore size) and degassed for about $2 \mathrm{~h}$ under constant stirring. 
Table 2.2: Overview of buffer system used to establish solid supported lipid membranes.

\begin{tabular}{|c|c|c|}
\hline Buffer system & Composition & \\
\hline $\begin{array}{l}\text { Phosphate buffered saline } \\
\text { (PBS--) }\end{array}$ & $\begin{array}{l}137 \mathrm{~mm} \mathrm{NaCl}, 2.7 \mathrm{~mm} \mathrm{KCl} 8.1 \mathrm{~mm} \\
\mathrm{Na}_{2} \mathrm{HPO}_{4}, 1.5 \mathrm{~mm} \mathrm{KH}_{2} \mathrm{PO}_{4}\end{array}$ & pH 7.4 \\
\hline $\mathrm{Ca}^{2+}$-spreading-buffer & $20 \mathrm{~mm}$ Tris/HCl, $50 \mathrm{~mm} \mathrm{KCl}, 1 \mathrm{~mm} \mathrm{CaCl} 2$ & $\mathrm{pH} 7.4$ \\
\hline $\mathrm{Ca}^{2+}$-measurement-buffer & $20 \mathrm{~mm}$ Tris/HCl, $50 \mathrm{~mm} \mathrm{KCl,} 10 \mathrm{~mm} \mathrm{CaCl}_{2}$ & $\mathrm{pH} 7.4$ \\
\hline $\begin{array}{l}\text { EDTA-measurement- } \\
\text { buffer }\end{array}$ & $20 \mathrm{~mm}$ Tris/HCl, $50 \mathrm{~mm} \mathrm{KCl,} 2 \mathrm{~mm}$ EDTA & $\mathrm{pH} 7.4$ \\
\hline
\end{tabular}

Table 2.3: Overview of lipid mixtures used to establish solid supported lipid membranes.

\begin{tabular}{ccccc}
\hline \hline $\begin{array}{c}\text { POPC / } \\
\text { mol\% }\end{array}$ & $\begin{array}{c}\text { Lyso-LacCer / } \\
\text { mol\% }\end{array}$ & $\begin{array}{c}\text { GM3 / } \\
\text { mol\% }\end{array}$ & $\begin{array}{c}\boldsymbol{\beta} \text {-Bodipy } \\
\text { mol\% }\end{array}$ & $\begin{array}{c}\text { Texas RedTM DHPE/ } \\
\text { mol\% }\end{array}$ \\
\hline \hline 99 & - & - & 1 & - \\
99 & - & - & - & 1 \\
89 & 10 & - & 1 & - \\
98 & 1 & - & 1 & - \\
97 & 2 & - & 1 & 1 \\
89 & - & 10 & - & \\
\hline
\end{tabular}

The concentrations $c$ of the stock solutions of the matrix phospholipid and phospholipid-coupled dyes, dissolved in chloroform, were $c(\mathrm{POPC})=10 \mathrm{mg} / \mathrm{mL}$, $c\left(\beta\right.$-Bodipy $\left.{ }^{\mathrm{TM}}\right)=0.1 \mathrm{mg} / \mathrm{mL}$ and $c\left(\right.$ Texas Red $\left.{ }^{\mathrm{TM}} \mathrm{DHPE}\right)=1 \mathrm{mg} / \mathrm{mL}$. The solvents and concentrations of the glycosphingolipid stock solutions are listed in Table 2.4. All stock solutions were used without further purification or characterization (except from selected differential scanning calorimetry experiments).

Table 2.4: Overview of the solubility and stock solution concentrations $c$ of the used glycosphingolipids.

\begin{tabular}{|c|c|c|}
\hline & Solvent & $c / \mathrm{mg} \cdot \mathrm{mL}^{-1}$ \\
\hline LacCer & $\begin{array}{l}\text { Chloroform/methanol/ultra-pure water } \\
\text { (5:1:0.1 by vol. })^{104}\end{array}$ & 1 \\
\hline Lyso-LacCer & $\begin{array}{l}\text { Chloroform/methanol/ ultra-pure water } \\
\qquad(2: 1: 0.1 \text { by vol. })^{105}\end{array}$ & 1 \\
\hline $\mathbf{G}_{\mathbf{M} 3}$ & $\begin{array}{l}\text { Chloroform/methanol/ ultra-pure water } \\
\text { (5:4:1 by vol. })^{94}\end{array}$ & 1 \\
\hline
\end{tabular}


In order to prepare lipid films, test tubes were rinsed with $500 \mu \mathrm{L}$ methanol p.a. (SIGMA-ALDRICH ${ }^{\circledR}$, St. Louis, MO, USA) and dried under a constant stream of nitrogen at room temperature. The respective volumes of the different lipid stock solutions were pipetted into the test tubes filled with $100 \mu \mathrm{L}$ of chloroform (w/o amylene, SigmAALDRICH $^{\circledR}$, St. Louis, MO, USA) according to the desired ratios (total lipid mass: $0.6 \mathrm{mg}$ ). The solvent in the test tubes was evaporated under a gentle stream of nitrogen for 20 minutes and further dried under reduced pressure for 3-4 h at the highest main phase transition temperature of the used lipids and stored at $4{ }^{\circ} \mathrm{C}$ until use. For the preparation of SUVs, the dried lipid films were mixed with $600 \mu \mathrm{L}$ degassed phosphate buffered saline (PBS--) buffer (cf. Table 2.2) and rehydrated for $30 \mathrm{~min}$ above the main phase transition temperature of the corresponding lipid. The total lipid concentration in the solution was $c=1.00 \mathrm{mg} / \mathrm{mL}$. Subsequently, the lipid suspension was vortexed two times for $60 \mathrm{~s}$ at intervals of two minutes resulting in a detaching of the lipids from the test tube wall. The resulting multilayered vesicles were transferred to micro test tubes (1.5 mL, EPPENDORF, Hamburg, Germany) and sonicated in an ultra-sonic homogenizer (Sonopuls bath sonifier, BANDELIN, Berlin, Germany) for 30 min (4 cycles, $65 \%$ intensity) to obtain unilamellar vesicles. The produced SUVs were used directly. 


\subsection{DIFFERENTIAL SCANNING CALORIMETRY}

Differential scanning calorimetry (DSC), developed in 1962 by Watson and Neil106,107, deals with the analysis of temperature-induced changes in specific material properties. To identify temperature changes, the heat flux of the sample is measured during its heating or cooling process over a certain period of time. In this thesis, DSC is used to study the thermochroic behavior and with this the main phase transition temperature of the glycosphingolipids LacCer and lyso-LacCer.

\subsubsection{SETUP AND MEASURING PRINCIPLE}

The change in enthalpy can be determined by the principle of a heat flux (cf. Figure $2.7[\mathrm{~A}]$ ) or a power-compensation DSC (cf. Figure 2.7 [B]).

[A]

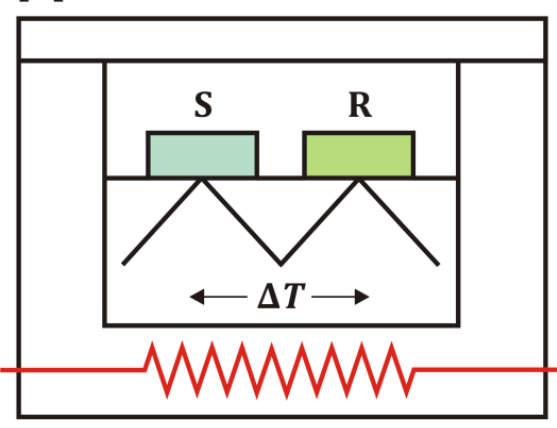

Single heat source
[B]

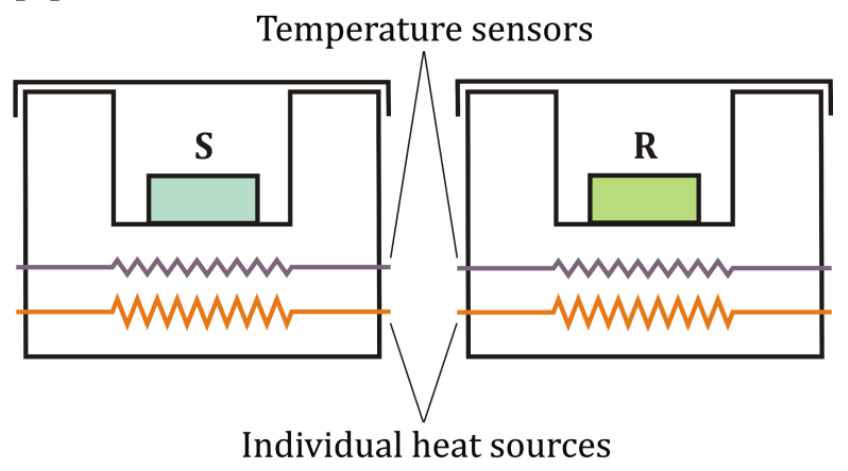

Figure 2.7: Schematic drawings of differential scanning calorimetry (DSC) set-ups. Part [A] shows a heat flux DSC, consisting of sample (S, blue) and reference (R, green) chambers, whereas in [B] a power-compensate DSC is shown. Here, sample (S, blue) and reference (R, green) chambers are heated up individually. ${ }^{\mathbf{1 0 8}}$

The latter consists of a sample (S, blue) and a reference (R, green) measuring chamber, which are located in separate ovens and are coupled by their respective electrical heating elements. By means of temperature sensors, the temperature difference between the ovens is set to $\Delta T=0$ during the controlled heating or cooling procedure. The enthalpy change of the sample is determined by an increase of the heating capacity in the sample chamber for an endothermic process, whereas it is reduced in an exothermic process.

The heat flux DSC consists of sample and reference measuring chambers located in a symmetrical furnace, which are connected by an integrated temperature sensor. Thus, the temperature difference between sample and reference as well as the respective 
absolute temperature can be determined and differences in the heat flow can be detected. ${ }^{108-110}$

\subsubsection{EXPERIMENTAL PROCEDURE}

To analyze the main phase transition temperature of the GSLs LacCer and lyso-LacCer, differential scanning calorimetry measurements were carried out using a MicroCal VP-DSC calorimeter (MALVERN INSTRUMENTS LTD., Malvern, United Kingdom). ${ }^{1}$

\section{Preparation of mULtilamelLaR Vesicles}

In order to determine the gel-to-liquid main phase transition temperature of the GSLs LacCer and lyso-LacCer, lipid films were prepared according to the protocol described in chapter 2.2.3.1. For the preparation of multilamellar vesicles (MLVs), the dried lipid films were dissolved in degassed DSC-measurement-buffer containing 20 mм Hepes, $100 \mathrm{~mm} \mathrm{NaCl}$ and $10 \mathrm{~mm}$ EDTA and rehydrated for $30 \mathrm{~min}$ at $90^{\circ} \mathrm{C}$ for LacCer and at $70{ }^{\circ} \mathrm{C}$ for lyso-LacCer. The resulting lipid concentrations were $c$ (LacCer) $=0.5 \mathrm{mg} / \mathrm{mL}$ and $c$ (lyso-LacCer) $=2 \mathrm{mg} / \mathrm{mL}$. The lipid suspensions were vortexed two times for $60 \mathrm{~s}$ in intervals of two minutes resulting in a detaching of the lipids from the test tube wall. The multilayered vesicles were transferred to $1.5 \mathrm{~mL}$ micro test tubes, degassed, and used directly for DSC measurements.

\section{DSC SETTINGS AND PARAMETERS}

All settings and parameters used during DSC measurements are listed in

Table 2.5. The examination of the main phase transition temperature is performed by the data analysis software Origin ${ }^{\circledR}$ (ORiginLAB CoRPORATION, Northampton, MA, USA) that was already implemented into the DSC measurement software.

\footnotetext{
${ }^{1}$ Kindly provided by Prof. Dr. Jörg Enderlein, Third Institute of Physics, Georg-August University, Göttingen, Germany.
} 
Table 2.5: Overview of parameters used in DSC experiments.

\begin{tabular}{ccc}
\hline Parameters & LacCer & Lyso-LacCer \\
\hline \hline Lipid concentration $/ \mathrm{mg} \cdot \mathrm{mL}^{-1}$ & 0.5 & 2 \\
Scan rate $/{ }^{\circ} \mathrm{C} \cdot \mathrm{h}^{-1}$ & 15 & 15 \\
Number of scans & 3 & 3 \\
Starting temperature $/{ }^{\circ} \mathrm{C}$ & 20 & 20 \\
Final temperature $/{ }^{\circ} \mathrm{C}$ & 90 & 90 \\
Post cycle temperature $/{ }^{\circ} \mathrm{C}$ & 10 & 10 \\
Prescan thermostat & 15 & 15 \\
Postscan thermostat & 15 & 15 \\
\hline
\end{tabular}

\subsection{SOLID SUPPORTS FOR LIPID MEMBRANES}

For the investigation of biological membranes in vitro, different types of model membrane systems have been developed mimicking the natural composition of cellular plasma membranes. This allows targeted investigations of individual membrane components or studies of lipid rafts or GEMs (cf. Chapter 1.2). Following first experiments by MCCONNELL and TAMM111,112, solid supported lipid membranes (SSLMs) have become one of the most important tools in the past four decades for simulating the plasma membrane of cells. These artificial systems offer the great advantage of a known composition and organization under equal conditions, which can be analyzed by common surface-based analytical techniques ${ }^{10}$ or function as biosensors. ${ }^{113}$ SSLMs can be prepared by Langmuir-type applications or vesicle spreading approaches ${ }^{10,114}$ on different substrates like glass, silicon dioxide, mica or titanium oxide ${ }^{112,115-118}$ offering mechanical stability. ${ }^{119}$ For vesicle spreading approaches, small unilamellar vesicles (SUV, $d<100 \mathrm{~nm}$ ) and large unilamellar vesicles (LUVs, $100<d<1000 \mathrm{~nm}$ ) are mostly used to prepare SSLMs, while giant unilamellar vesicles (GUVs, $d \geq 1 \mu \mathrm{m}$ ) are used to produce pore-spanning lipid membranes. ${ }^{120}$

SSLMs are not directly coupled to the respective underlying hydrophilic substrate but are separated from it by an approximately 1-2 nm thick water layer. ${ }^{121,122}$ Still, the 
leaflet facing the solid support often behaves less mobile. Consequently, SSLMs were valuable for the exploration of the lateral mobility and the phase behavior of incorporated lipids.

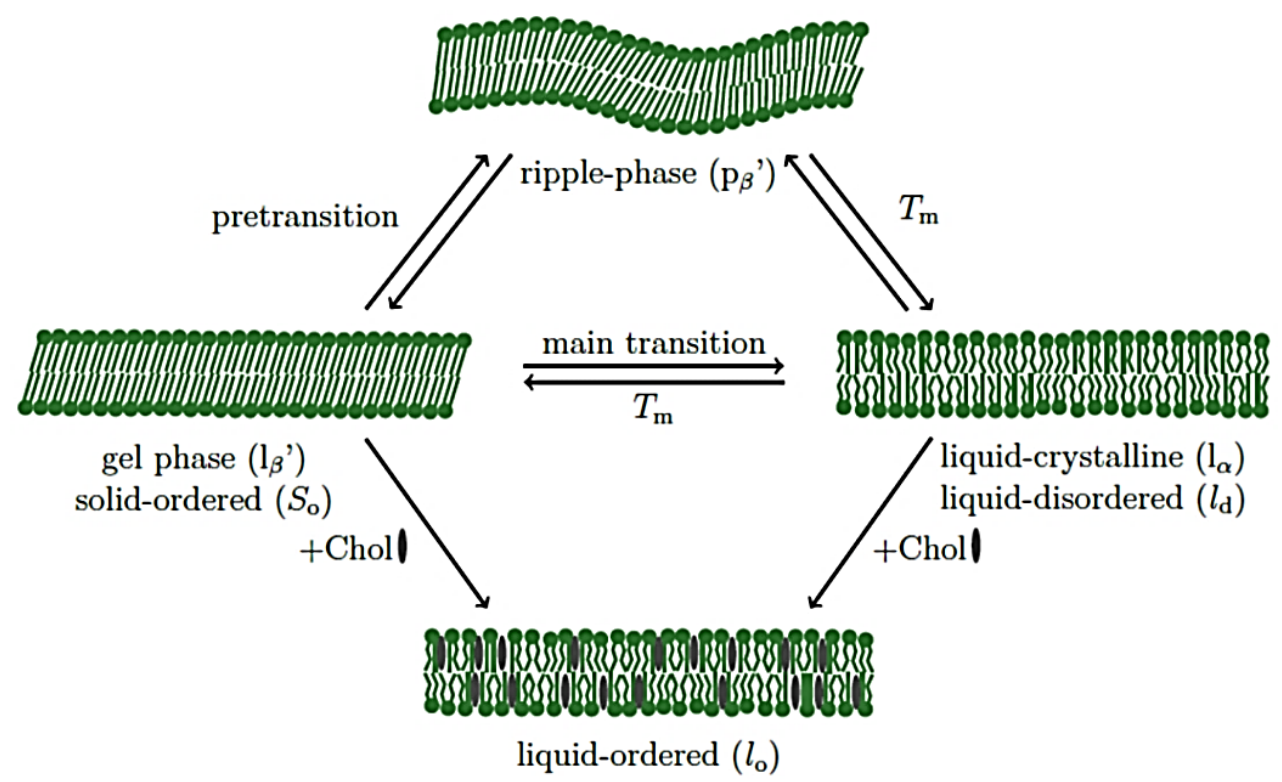

Figure 2.8: Schematic illustration of lipid membranes showing different lamellar phase behavior. ${ }^{\mathbf{1 2 3 , 1 2 4}}$ Depending on temperature, pressure or lipid composition, lipid membranes can form gel $\left(\mathrm{l}_{\beta}{ }^{\prime}\right)$ or solid-ordered (So), ripple $\left(\mathrm{p}^{\prime}{ }^{\prime}\right)$ as well as liquid crystalline $(l \alpha)$ or liquid-disordered $(l d)$ phases. The incorporation of cholesterol leads to the formation of liquid-ordered (lo) phases. $T_{\mathrm{m}}$ describes the specific main phase transition temperature of the respective lipid.

Depending on temperature, pressure or lipid composition, lipid membranes show different lamellar phase behavior (cf. Figure 2.8). 35,125,126 The specific phase transition is influenced by the symmetry and the composition of the hydrocarbon chains of the lipids and their degree of saturation. ${ }^{1}$ Long hydrocarbon chains and a high degree of saturation (trans configuration) result in enhanced van-der-Waals forces due to improved interactions between the lipids. ${ }^{123}$ This in turn restricts the mobility of the lipids and more energy is required to initiate a phase transition. ${ }^{1}$ In this so-called gel $\left(l^{\prime}\right)$ or solid-ordered $\left(S_{0}\right)$ phase, the lipids are tilted by an angle of $30^{\circ}$ to the membrane normal. ${ }^{123}$ Increasing the temperature promotes the phase transition from the $S_{\mathrm{o}}$ phase to the ripple phase $\left(\mathrm{p}_{\beta}^{\prime}\right)$, a pretransition phase, which is characterized by an asymmetrically wavy surface structure. ${ }^{123}$ If the ambient temperature is further increased and the lipid-specific main phase transition temperature $T_{\mathrm{m}}$ is reached, the liquid crystalline $\left(l_{\alpha}\right)$ or liquid-disordered $\left(l_{d}\right)$ phase is formed. Here, highly mobile membrane lipids with disordered hydrocarbon chains are arranged parallel to the membrane normal and form planar membranes. ${ }^{123}$ The additional incorporation of cholesterol to the lipid membrane (liquid-ordered $\left(l_{0}\right)$ phase) does not change the 
specific $T_{\mathrm{m}}^{126}$ but it leads to a disruption of the high density packing of the lipids present in the $S_{0}$ phase, incresing the order of lipids in the $l_{\mathrm{d}}$ phase. ${ }^{123}$

In order to analyze the GSLs-incorporated SSLMs and the impact of the carbohydratedriven adhesion of the B16 carcinoma mouse cell line, three different substrates were used.

\subsubsection{GLASS SUBSTRATES}

Due to their ease of handling, the universal functionalization options and their transparency, glass substrates are widely used solid supports, especially in optical analysis methods. To perform optical microscopy experiments and force spectroscopy studies, glass bottom petri dishes (MATTEK CoRPORATION, Ashland, MA, USA) were used. In order to prepare a homogenous lipid membrane on glass substrates or to provide a clean surface for cell attachment experiments, the petri dishes were incubated in a $1 \%$ Hellmanex $^{\circledR}$ III (HELlma ANALYTICS, Müllheim, Germany) solution at room temperature overnight, rinsed ten times with ultra-pure water and dried under a gentle stream of nitrogen. In order to prepare solid supported lipid membranes onto the cleaned and hydrophilized glass bottom petri dishes, the supports were heated to the specific main phase transition temperature of the incorporated lipid if necessary. The glass substrates are the primary used supports for atomic force microscopy, as well as for fluorescent and optical microscopy techniques. Further information about the preparation techniques and experimental parameters are described in the respective Chapters 2.6.1 and 2.6.2.

\subsubsection{BOROSILICATE GLASS MICROSPHERES}

For colloidal probe microscopy, a borosilicate glass microsphere (DUKE SCIENTIFIC Corporation, Palo Alto, Canada) with a diameter of $15 \mu \mathrm{m}$ was glued to a tip-less cantilever and enveloped with a SSLM. With this approach, the binding affinity between the GSLs Gм3 and lyso-LacCer can be obtained. Further information about the preparation techniques and experimental parameters are described in chapter 2.6.2.1. 


\subsubsection{SILICON DIOXIDE SUBSTRATES}

$\mathrm{SiO}_{2}$ substrates (CRYSTEC, Berlin, Germany) with an oxide layer thickness of $100 \mathrm{~nm}$ were selected to determine the topography of glycosphingolipid doped membranes. The $\mathrm{SiO}_{2}$ supports are characterized by a low roughness compared to glass substrates. This allows visualization the surface topography of the membranes. The $\mathrm{SiO}_{2}$ substrates were cut $(1.5 \mathrm{~cm}$ x $2.0 \mathrm{~cm})$, cleaned with ultra-pure water and ethanol p.a. (SIGMA-ALDRICH ${ }^{\circledR}$, St. Louis, MO, USA) and placed in a teflon holder. Subsequently, they were oxidized for $45 \mathrm{~min}$ at $70{ }^{\circ} \mathrm{C}$ in a solution prepared using ultra-pure water, $30 \%$ hydrogen peroxide $\left(\mathrm{H}_{2} \mathrm{O}_{2}\right.$, SIGMA-ALDRICH ${ }^{\circledR}$, St. Louis, MO, USA) and $25 \%$ ammonia solution $\left(\mathrm{NH}_{3}\right.$, Sigma-ALDRICH ${ }^{\circledR}$, St. Louis, MO, USA) with a ratio of 5:1:1 by volume. Next, the supports were thoroughly rinsed with ultra-pure water, dried in a gentle stream of nitrogen, treated for $1 \mathrm{~min}$ in $\mathrm{O}_{2}$ plasma, and stored in ultra-pure water until use. If necessary, the $\mathrm{SiO}_{2}$ supports were heated to the main phase transition temperature of the specific lipid to ensure incorporation of GSLs in SSLMs.

\subsection{ATOMIC FORCE MICROSCOPY}

The invention of the scanning tunneling microscopy (STM)127,128 in 1981 allowed for the first time to image surfaces at atomic resolution. However, this microscopy technique was still limited to the use of conducting samples as it is depended on the measurement of the tunneling current between an electric sensor and the surfaces of metals, semiconductors and superconductors. ${ }^{129}$ Based on STM, BINNIG, QUATE and GERBER $^{130}$ developed the atomic force microscopy (AFM) in 1986, a powerful tool to study the surface topographies of different samples in the nanometer range. The lateral resolution can range from $0.1 \mathrm{~nm}$ to $10 \mathrm{~nm} .{ }^{131}$ The ability to carry out measurements in air or in liquids makes AFM a versatile instrument in biology, biochemistry or medicine as this technique can be used to work under physiological conditions. Consequently, AFM has become an effective and widely used method for the analysis of surfaces over the last 30 years. In addition to the possibility of obtaining topographical images of a sample, there are a number of methods that have 
been designed to investigate individual molecular interactions by means of force spectroscopy.

\subsubsection{SETUP AND MEASURING PRINCIPLE}

The principle of AFM is the measurement of a force between a local probe, the socalled cantilever, and the surface of a sample. A general set-up is illustrated in Figure 2.9. Surface forces between $10^{-12} \mathrm{~N}$ and $10^{-4} \mathrm{~N}$ can be measured 132,133 , including electrostatic, hydrodynamic or van-der-Waals forces. Besides, this microscopy technique overcomes the resolution of Abbe holding a lateral resolution in the angstrom region. The most important component of the AFM is the cantilever, which is made of silicon or silicon nitride and consists of a sharp, a few micrometers long tip. The various forms of the cantilever affect its resonances and spring constants, through which the cantilever receives its special characteristics. Cantilever with small spring constants record even the smallest changes in the interaction between cantilever tip and sample surface and are therefore considered to be very sensitive.

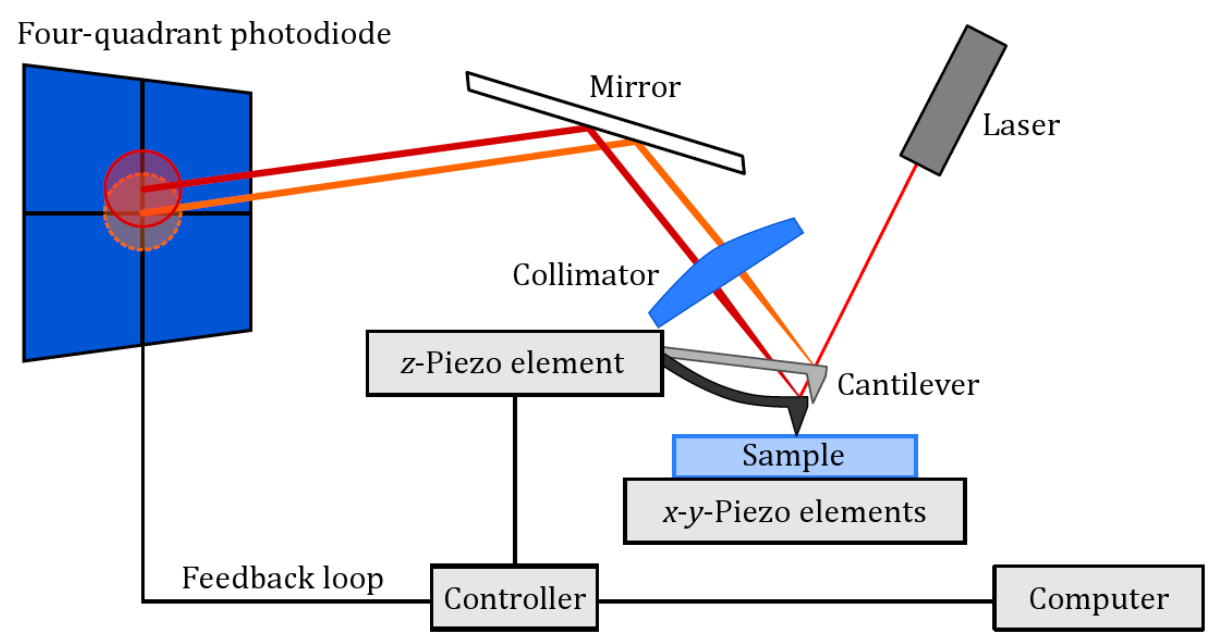

Figure 2.9: Schematic drawing of an atomic force microscope.134 In this setup, a laser beam is positioned on the back of a cantilever. Its reflection is directed via a collimator and mirror to a four-quadrant photodiode. A $\boldsymbol{z}$-piezo element varies the distance between the tip and the surface, whereas the horizontal movement of the tip over the sample is made possible by $\boldsymbol{x}$ - $\boldsymbol{y}$-piezo elements. The piezo elements are managed via a controller, which is connected to a computer.

The deflection of the cantilever can be detected by means of a laser, which is adjusted to the back of the cantilever and reflected on a four-quadrant photodiode. The calibration of the cantilever (cf. chapter 2.6) allows to convert the voltage signal of the photo diode into the force that acts on the cantilever. With the help of a $z$-piezo element the distance between the tip and the surface can be adjusted very precisely. 
A horizontal movement of the tip over the sample is made possible by $x$ - $y$-piezo elements.

\subsection{FORCE SPECTROSCOPY}

With the help of force spectroscopy, different surface forces can be measured and analyzed. Using the vertical movement of the $z$-piezo element and the resulting movement of the cantilever, force-distance curves can be obtained.

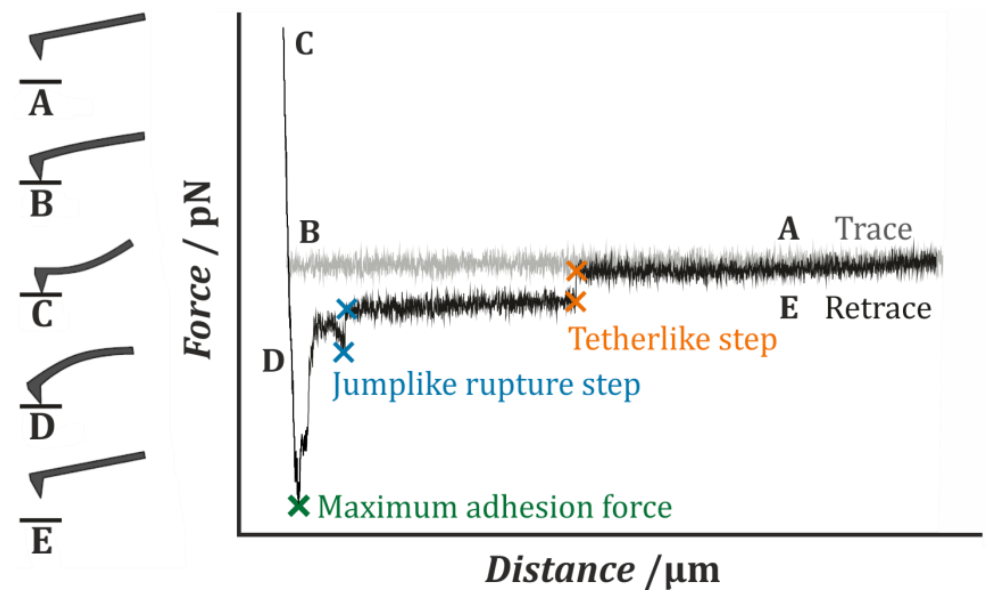

Figure 2.10: Schematic illustration of a force-distance curve during the retraction process. From [A] to [B], the cantilever approaches the surface of the sample (trace, dashed line) until a contact between both is formed [B]. In point $[\mathrm{C}]$ a certain setpoint is reached, which causes the cantilever to be retracted from the surface. During the retraction procedure [D], the maximum adhesion force (green cross) can be followed by individual force steps, such as jumplike rupture steps (blue crosses) or tetherlike steps (orange crosses). In [D] the original position of the cantilever is reached again.

In Figure 2.10, a schematic illustration of a typical force-distance-curve is shown. From point $[\mathrm{A}]$ to $[\mathrm{B}]$, the cantilever approaches the surface of the sample. Since there are no forces acting between the sample and the cantilever during this approach, there is no measurable deflection of the cantilever. At point [B], the cantilever comes in contact with the surface, resulting in an increased force on the sample surface and in a bending of the cantilever. If a defined force, the so-called setpoint, is reached [C], the cantilever is retracted from the surface by a piezo element. The cantilever is still held on the surface of the sample during this process by specific adhesion forces and thus experiences a negative deflection [D]. If a critical maximum adhesion force is reached, the cantilever starts to detach from the surface. In some cases unbinding effects occur including jumplike rupture steps ${ }^{135,136}$, which are characterized by a non-linear force increase before an instantaneous force decrease or tetherlike 
steps ${ }^{135,136}$, which show long plateaus of constant forces before also instantaneous force decreases occur. These forces are assigned to the breaking of single or grouped bonds between the cantilever and the sample or membrane nanotubes, which are pulled out of the cellular surface. At point [E], the cantilever has returned to its original position, where it experiences no deflection or forces because there is no contact with the surface anymore.

Force-distance curves show the force $F$ that results from the attractive and repulsive interactions between the cantilever tip and the sample surface as a function of their distance $d$. By subtracting the cantilever deflection $Z_{c}$ from the total movement of the piezo element, the distance $d$ between the cantilever tip and the sample surface can be determined. If the specific spring constant $k_{c}$ of the cantilever is known Hook's law can be applied for the following relationship for the measured force $F$ :

$$
F=-k_{c} \cdot Z_{c}
$$

\section{Calibration Procedure of the CANTILEVER}

As described above, the specific spring constant of a cantilever is needed to determine the force and hence the binding strength between two or more molecules. Generally, cantilever with spring constants between $0.005 \mathrm{~N} / \mathrm{m}$ and $40 \mathrm{~N} / \mathrm{m}$ are commercially available. However, since the spring constant is dependent on the manufactured shape and size of the cantilever, it has to be calibrated before each measurement in order to calculate the force. For this purpose, the thermal noise method is used. First, the cantilever is pressed on a clean, hard substrate and a force-distance curve is recorded. From the slope of the contact region of the curve, the sensitivity in units of $\mathrm{nm} / \mathrm{V}$ can be obtained to convert the photo diode signal into the cantilever bending. By recording the thermal noise of the cantilever, the specific spring constant can be determined. ${ }^{132,137}$ 


\subsubsection{SINGLE-CELL FORCE SPECTROSCOPY}

In addition to being able to examine the cellular adhesion strength by micropipettes ${ }^{138}$ or beads using a laser beam ${ }^{139}$, single-cell force spectroscopy (SCFS) has become a major tool in analyzing the adhesion properties of living cells within the last decades. The combination of an atomic force and an optical microscope allows not only probing the cell with functionalized surfaces or different cells, but also the interactions between components on the outer leaflet of cellular plasma membranes. Within this thesis, SCFS based on AFM is used to determine the maximum adhesion force of $\mathrm{G}_{\mathrm{M} 3}$-overexpressing B16 carcinoma cells of different malignancy on lysoLacCer-containing SSLMs with variable parameters (cf. Table 2.6, Chapter 2.6.1.1). For this purpose, a single B16-F1 or B16-F10 carcinoma cell was attached to a poly-Dlysine coated tip-less cantilever and brought into contact with the lipid membrane. The recorded force-distance curve gives a direct measure of the adhesion forces, which are presumably due to the interaction between the GSL moieties Gм3 and lysoLacCer. Figure 2.11 illustrates the set-up of the SCFS experiment used within this thesis. The approach and retraction of the cell-attached cantilever and its calibration are described in Chapter 2.6.
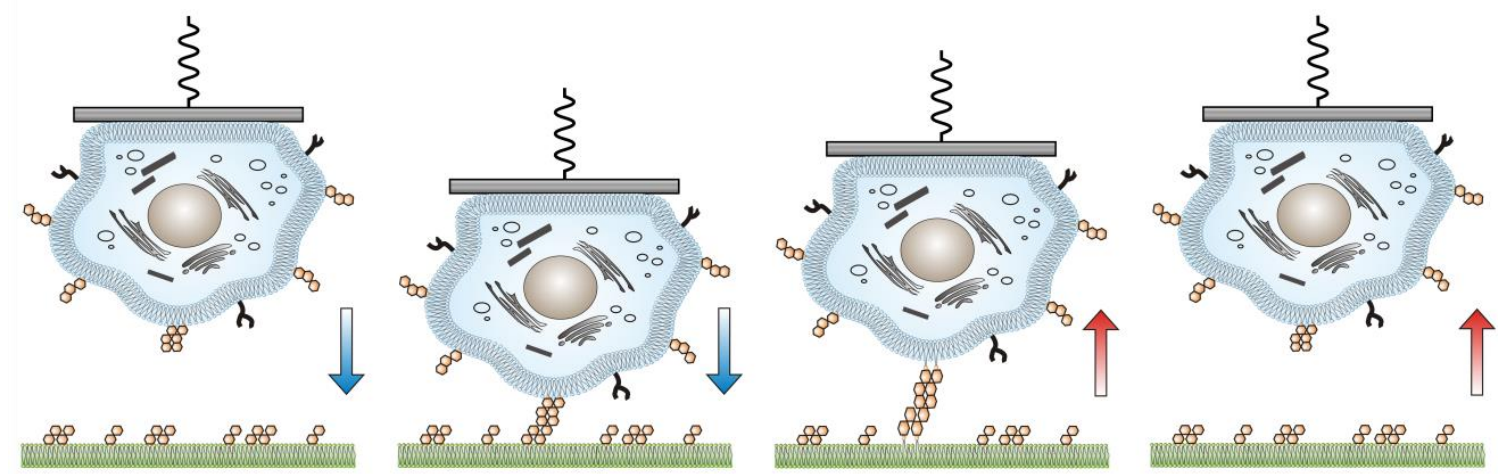

Figure 2.11: Schematic drawing of a single-cell force spectroscopy set-up. From left to right the approach (blue arrow) and retraction (red arrow) process of a $\mathrm{G}_{\mathrm{M} 3}$-overexpressing B16 cell (blue) attached to a cantilever (grey bar with spring) onto a lyso-LacCer-containing solid supported lipid membrane (green) is shown. GM3 is represented by three, while lyso-LacCer is illustrated by two adjacent orange-colored six-membered ring structures.

\subsubsection{EXPERIMENTAL PROCEDURE}

All force spectroscopy measurements were carried out with a Cellhesion ${ }^{\circledR} 200$ AFM (JPK InSTRUMENTS, Berlin, Germany), which was combined with an inverted IX 81 optical microscope (OLYMPUS, Shinjuku, Japan) using a UPLFLN 2 (10x magnification, $N A=0.3$, OLYMPUS, Shinjuku, Japan) or UPLFLN (40x magnification, $N A=0.75$, 
OLYMPUS, Shinjuku, Japan) objective to analyze the correct position of the cell on the cantilever. The fluorescent-labeled solid supported lipid membranes were examined with an upright BX 51 optical microscope (OLYMPUS, Shinjuku, Japan) using a LUMPLFLN (40x magnification, $N A=0.8$, OLYMPUS, Shinjuku, Japan) objective or a FluoView FV1200 confocal laser scanning microscope (OLYMPUS, Shinjuku, Japan) for its integrity and homogeneity.

\section{FUNCTIONALIZATION PROCEDURE OF THE CANTILEVER}

A tip-less silicon cantilever (Arrow-TL2-50, NANoWorLd, Neuenburg, Switzerland) with a nominal spring constant of $k_{c}=0.03 \mathrm{~N} / \mathrm{m}$ was used to perform single-cell force experiments. Before functionalization, the cantilever was cleaned with ethanol p.a. mixed with ultra-pure water (60:40 by vol.) and isopropanol p.a. for about 5 min each. Afterwards they were cleaned in an argon plasma for $1 \mathrm{~min}$, incubated in $1 \mathrm{~mL}$ of a $100 \mu \mathrm{g} / \mathrm{mL}$ poly-D-lysine (PDL, in PBS--, cf. Table 2.2) solution for $15 \mathrm{~min}$ and gently washed 10 times with ultra-pure water. PDL is a positively charged proteinogenic $\alpha$ amino acid and assures the attachment of a cell to its surface. The functionalized cantilever were used directly or stored at $4{ }^{\circ} \mathrm{C}$ for a maximum of one week. The coating process was performed according to a method proposed by DZEMENTSEI et al. 140

\section{PREPARATION OF SOLID SUPPORTED LIPID MEMBRANES}

For SCFS experiments, SSLMs consisting of POPC, lyso-LacCer and $\beta$-Bodipy ${ }^{\mathrm{TM}}$ (89:10:1 mol\%) were prepared. For control experiments SSLMs with less lyso-LacCer (POPC/lyso-LacCer/ $\beta$-Bodipy ${ }^{\mathrm{TM}}$, 98:1:1 mol\%) as well as SSLMs with lacking lysoLacCer (POPC/ $\beta$-Bodipy ${ }^{\mathrm{TM}}$, 99:1 mol\%) were used (cf. Table 2.3).

For SCFS, $35 \mathrm{~mm}$ glass bottom petri dishes with a $14 \mathrm{~mm}$ glass microwell (No. 1.5, MatTeK Corporation, Ashland, MA, USA) were used as a support for the SSLMs (cf. chapter 2.4). To separate the glass microwell from the surrounding area of the petri dish, a liquid blocker (Super PAP Pen Liquid Blocker, mini, DAIDO SANGYo Co., LTD, Tokyo, Japan) was applied to the outer edge of the glass microwell.

In order to prepare solid supported lipid membranes on glass substrates, $60 \mu \mathrm{L}$ of a $1 \mathrm{mg} / \mathrm{mL}$ SUV solution (cf. chapter 2.2.3.1) were pipetted onto the inner glass microwell. The solution was incubated for $10 \mathrm{~min}$ before $320 \mu \mathrm{L}$ of degassed $\mathrm{Ca}^{2+}$ 
spreading-buffer (cf. Table 3.1) were added to the SUV solution, resulting in a lipid concentration of about $0.15 \mathrm{mg} / \mathrm{mL}$. Each step described above was carried out at the main phase transition temperature of the highest melting lipid (cf. chapter 2.2.1) in a heating oven (BINDER, Tuttlingen, Germany). The maintenance of the correct temperature was also applied to buffer solutions and materials used to prepare SSLMs.

Due to the fact that the lipids used in this thesis vary in their main phase transition temperature, there is a slight difference in performing the spreading process for lipids with a high transition temperature compared to those which are already in the liquid phase at room temperature. For lipids with a main phase transition temperature above room temperature (LacCer, lyso-LacCer, $\mathrm{G}_{\mathrm{M} 3}$ ) the spreading process was performed for one hour at the desired temperature before the formed lipid membrane was slowly cooled to room temperature in the heating oven overnight. This cooling process was intended to avoid sudden temperature differences and thus to ensure the formation of an intact, defect-free lipid membrane. After the overnight incubation process, the whole sample was gently rinsed five times with $1 \mathrm{~mL}$ of degassed PBS--buffer (cf. Table 2.2) to remove non-spread vesicles. By using the liquid blocker, it was possible to remove the PBS---buffer on the outer plastic rim of the petri dish to prevent the lipid membrane prepared on the microglass from drying. Subsequently, the rim was passivated with $1 \mathrm{~mL}$ of a triton-free blocking solution containing BSA (cf. Table 2.1) for 1-2 hours. BSA is a stable, inert protein that blocks non-specific binding sites, therefore preventing tight attachment or spreading of cells. The BSA-containing outer rim was gently washed three times with $1 \mathrm{~mL}$ of degassed PBS-- buffer (cf. Table 2.2). Finally, the entire sample was washed three times with $1 \mathrm{~mL}$ of D10F+ medium (cf. Table 2.1) before cell addition.

For lipids that are characterized by a low main phase transition temperature (POPC), the spreading process after SUV addition was performed for one hour at room temperature before the formed lipid membranes were gently rinsed with degassed PBS-- buffer and $\mathrm{D}^{-} 10 \mathrm{~F}^{+}$medium as described above. Before the actual SCFS experiment, the SSLMs were examined for integrity and directly used or stored at $4{ }^{\circ} \mathrm{C}$ for a maximum of $24 \mathrm{~h}$. 


\section{B16 CELL LINE FUNCTIONALIZATION}

For single-cell force studies, the B16-F1 (low metastatic potential) and the B16-F10 (strong metastatic potential) cells were detached from the bottom of the cell culture flask using the trypsinization procedure (cf. Chapter 2.1.1) and were then taken up in $1 \mathrm{~mL}$ of warm D10F+ medium (cf. Table 2.1). After counting, approximately 500,000 cells were transferred to $500 \mu \mathrm{L}$ of warm D10F $\mathrm{F}^{+}$medium. For the SCFS measurements, 20,000 cells were directly added to the BSA-functionalized plastic rim of the petri dish and allowed to settle down for $1 \mathrm{~min}$. Then, a single cell was attached to a PDL-coated cantilever by approaching the cantilever to the cell surface (see below). For control experiments, the cells were treated with an anti-GM3 monoclonal IgM antibody or the enzyme sialidase according to the functionalization protocol in chapter 2.1.2.2 and likewise attached to the cantilever.

The untreated or functionalized cells were attached to a PDL-coated cantilever by performing a force-distance experiment on a chosen cell with the parameters given in Table 2.6. The dwell time of $30 \mathrm{~s}$ allowed the cells to attach to the cantilever tip. Afterwards, the cell-loaded cantilever was moved to regions containing the lipid membrane and pressed onto the membrane with varying dwell times. Using the same cell, 50 total force curves with 5 force curves on 10 different spots on the membrane were examined.

\section{AFM SETTINGS AND PARAMETERS}

Before performing the SCFS measurements, the PDL-coated cantilever was calibrated and its specific spring constant obtained using the thermal noise method. In Table 2.6 [A] important parameters used for the calibration are listed, while in [B] the parameters for attaching a single cell onto the PDL-coated and calibrated cantilever are shown. In order to determine the maximum adhesion force between the single cell and the surface of the individual SSLMs, parameters listed in Table 2.6 [C] were used with varying dwell times of 0 s, 0.125 s, 0.25 s, 0.5 s, 1 s, 5 s, 10 s, 15 s, 30 s and $60 \mathrm{~s}$.

Table 2.6: Overview of parameters used for [A] cantilever calibration, [B] attaching a single cell to the cantilever and $[\mathrm{C}]$ obtaining adhesion forces from the single cell with the individual SSLMs for SCFS experiments. 


\begin{tabular}{ccccccc}
\hline \hline & Setpoint & Velocity & Dwell time & Pause time & $\begin{array}{c}\text { Sample } \\
\text { rate }\end{array}$ & z-length \\
\hline A & $2 \mathrm{~V}$ & $5 \mu \mathrm{m} / \mathrm{s}$ & $0 \mathrm{~s}$ & $0 \mathrm{~s}$ & $6 \mathrm{kHz}$ & $50 \mu \mathrm{m}$ \\
B & $1 \mathrm{nN}$ & $5 \mu \mathrm{m} / \mathrm{s}$ & $30 \mathrm{~s}$ & $0 \mathrm{~s}$ & $6 \mathrm{kHz}$ & $80 \mu \mathrm{m}$ \\
C & $1 \mathrm{nN}$ & $10 \mu \mathrm{m} / \mathrm{s}$ & $0 \mathrm{~s}-60 \mathrm{~s}$ & $5 \mathrm{~s}$ & $6 \mathrm{kHz}$ & $80 \mu \mathrm{m}$ \\
\hline
\end{tabular}

\section{DATA ANALYSIS}

To determine the maximum adhesion force between the GSLs GM3 and lyso-LacCer in the presence of calcium-ions, the obtained force-distance curves of the retraction processes were analyzed with the JPK SPM data processing software and imported into a home-written MATLAB script ${ }^{2}$.

The following analysis steps were performed using the JPK software:

1. Automatically subtract the baseline

2. Automatically adjust the $\mathrm{x}$ offset (contact point)

3. Correct height for cantilever bending with unsmoothed height (tip-sample separation)

Using the MATLAB script, baseline and offset were checked by eye and corrected if necessary before the maximum adhesion force was evaluated by selecting a point close to the maximum force. In case no adequate baseline correction was applicable, the force curves were rejected.

\subsubsection{COLLOIDAL PROBE MICROSCOPY}

Within this thesis, colloidal probe microscopy (CPM) was used to investigate the weak but highly specific interaction between $\mathrm{GM}_{\mathrm{M}}$ and lyso-LacCer. CPM provides the advantage of a controllable surface functionalization and sample geometry. ${ }^{141}$

While regular force spectroscopic experiments typically use cantilever with a sharp tip, a sphere is replacing the tip in CPM. In this case, a borosilicate glass microsphere with a diameter of $15 \mu \mathrm{m}$ was glued to a tip-less cantilever (see below). In order to study the interaction of glycosphingolipids in an environment mimicking the cell membrane, they were embedded in SSLMs. To mimic the Gм3-expressing B16 cell line,

\footnotetext{
2 Kindly provided by Dr. Ingo Mey, Institute of Organic and Biomolecular Chemistry, Georg-August University, Göttingen, Germany.
} 
the glass sphere was enveloped with a GM3-containing lipid membrane and brought into contact with another SSLM containing its receptor lipid lyso-LacCer.
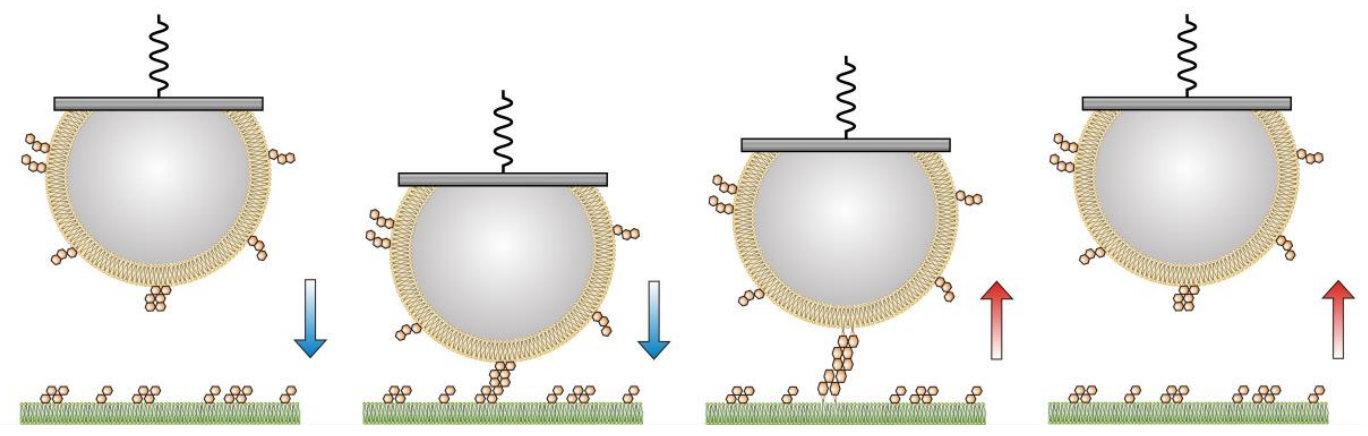

Figure 2.12: Schematic drawing of a colloidal probe microscopy set-up. From left to right the approach (blue arrow) and retraction (red arrow) process of a borosilicate glass microspheres enveloped with a $\mathrm{G}_{\mathrm{M} 3}$-containg lipid membrane (yellow) attached to a cantilever (grey bar with spring) onto a lyso-LacCer-containing solid supported lipid membrane (green) is shown. $\mathrm{G}_{\mathrm{M} 3}$ is represented by three, while lyso-LacCer is illustrated by two adjacent orange-colored six-membered ring structures.

Figure 2.12 illustrates the set-up of the CPM experiment used within this thesis. The approach and retraction of the sphere-attached cantilever and its calibration are described in Chapter 2.6.

\subsubsection{EXPERIMENTAL PROCEDURE}

All CPM measurements were carried out with a MFP-3D AFM (ASYLUM RESEARCH, Santa Barbara, CA, USA), which was combined with an inverted IX 51 optical microscope (OlYMPUS, Shinjuku, Japan) using a UPLFLN 2 (10x magnification, $N A=0.3$, OlYMPUS, Shinjuku, Japan) objective to analyze the correct position of the microsphere on the cantilever as well as the integrity of the fluorescently-labeled lipid membrane. The quality of the fluorescent-labeled lipid membrane on the glass support was examined with an upright BX 51 optical microscope (OLYMPUS, Shinjuku, Japan) using a LUMPLFLN (40x magnification, $N A=0.8$, OLYMPUS, Shinjuku, Japan) objective.

\section{FABRICATION OF COLLOIDAL PROBE CANTILEVER}

GM3-containing SSLMs were spread on borosilicate glass microspheres that have been previously glued to a triangular tip-less cantilever (MLCT-010, type C, BRUKER AFM Probes, Camarillo, Canada) with a nominal spring constant of $k_{c}=0.01 \mathrm{~N} / \mathrm{m}$. For this purpose, about $1 \mathrm{mg}$ of an Epikote 1004 epoxy resin (BREnNtaG GmBH, Mühlheim, Germany) with approximately the same amount of glass spheres were spread on a microscopy slide (VWR INTERNATIONAL, Radnor, PA, USA) and secured on a custom- 
made heating stage. The cantilever was fixed on a special holder of a MM3A-LS nanomanipulator (KLEINDiEK NANOTECHNiK GMBH, Reutlingen, Germany) by a doublesided adhesive strip and positioned such that the backside of the cantilever pointed to the microscope slide. Then, the heating stage was set to $110^{\circ} \mathrm{C}$ to achieve melting of the epoxy resin $\left(T_{M}=90^{\circ} \mathrm{C}^{142}\right)$. The instrument was designed in a way that the nanomanipulator could be moved by a joystick in order to bring the tip of the cantilever into contact with the liquid adhesive for a brief moment before it was placed onto a glass microsphere. In order to observe the attachment process of the sphere to the cantilever, a BX 51 optical microscope (OLYMPUS, Hamburg, Germany) was used. Finally, the correct position of the glued sphere was checked after a cooling time of a few minutes. Until use, the cantilever were stored at room temperature. Their functionality could be ensured for about 2-4 weeks before a detachment of the sphere under experimental conditions was observed.

\section{PREPARATION OF SOLID SUPPORTED LIPID MEMBRANES}

For CPM experiments, SSLMs consisting of POPC, GM3 and Texas Red ${ }^{\mathrm{TM}}$ DHPE (89:10:1 mol\%) on glass microspheres were prepared. On glass substrates, SSLMs contained POPC, lyso-LacCer and $\beta$-Bodipy ${ }^{\mathrm{TM}}$ (89:10:1 mol\%). For control measurements, the following SSLMs listed in Table 2.7 were prepared and brought in contact with each other.

Table 2.7: Overview of solid supported lipid membranes on glass microspheres and glass substrates for control experiments using colloidal probe microscopy. Lipid mixture ratios are given in mol\%.

SSLMs on glass microspheres

$$
\begin{aligned}
& \text { POPC/Gм3/Texas RedTM DHPE } \\
& \text { (89:10:1) } \\
& \text { POPC/GM3/Texas Red }{ }^{\mathrm{TM}} \text { DHPE } \\
& \text { (89:10:1) } \\
& \text { POPC/GM3/Texas Red }{ }^{\mathrm{TM}} \text { DHPE } \\
& \text { (89:10:1) } \\
& \text { POPC/ } \beta \text {-Bodipy }{ }^{\mathrm{TM}} \text { (99:1) }
\end{aligned}
$$

\section{SSLMs on glass substrates}




\section{Preparation OF SOLID SUPPORTED LIPID MEMBRANES ON GLASS MICROSPHERES}

To spread lipid membranes on the colloidal probe cantilever, it was mounted in the AFM and $80 \mu \mathrm{L}$ of a $1 \mathrm{mg} / \mathrm{mL}$ SUV solution (cf. chapter 2.2.3.1) with the desired lipid mixture were pipetted directly onto the glass microsphere that has been glued to a cantilever. The incubation period was $20 \mathrm{~min}$. It is important to mention that enough vesicle solution or buffer was added to the spherical membrane to prevent the lipid membrane from drying. Due to the fact that the colloidal probe cantilever needed to be mounted to the AFM beforehand, the spreading process could only be obtained at room temperature. After formation, the SSLMs were gently rinsed five times with $1 \mathrm{~mL}$ of degassed EDTA- or $\mathrm{Ca}^{2+}$-measurement-buffer (cf. Table 2.2, Chapter 2.2.3). The colloidal probe was directly used to perform CPM experiments.

\section{PREPARATION OF SOLID SUPPORTED LIPID MEMBRANES ON GLASS SUBSTRATES}

For performing model membrane studies using CPM, $50 \mathrm{~mm}$ glass bottom petri dishes with a 30 mm glass microwell (No. 1.5, MatTek Corporation, Ashland, MA, USA) were used (cf. chapter 2.4).

The preparation of SSLMs was similar to that for cell studies using SCFS (cf. chapter 2.6.1.1) with volumes adapted to the bigger radius of the glass microwell to obtain the same lipid concentration. For CPM, $500 \mu \mathrm{L}$ of a $1 \mathrm{mg} / \mathrm{mL}$ SUV solution (cf. chapter 2.2.3.1) were pipetted onto the inner glass microwell. The solution was likewise incubated for $10 \mathrm{~min}$ before $2 \mathrm{~mL}$ of degassed $\mathrm{Ca}^{2+}$-spreading-buffer (cf. Table 2.2, Chapter 2.2.3) were added to the SUV solution to maintain a lipid concentration of about $0.2 \mathrm{mg} / \mathrm{mL}$.). The formed SSLMs were gently rinsed five times with $1 \mathrm{~mL}$ degassed EDTA- or $\mathrm{Ca}^{2+}$-measurement-buffer (cf. Table 2.2, Chapter 2.2.3) to remove non-spread vesicles and finally checked for integrity. The SSLMs were directly used or stored at $4{ }^{\circ} \mathrm{C}$ for a maximum of $24 \mathrm{~h}$.

\section{AFM SETTINGS AND PARAMETERS}

Before CPM measurements were performed, the colloidal probe cantilever were calibrated as described in chapter 2.6. In Table 2.8 important parameters used for the calibration and determination of the maximum adhesion force between the GSLs GM3 and lyso-LacCer and control samples are listed. For each measurement, force maps 
with a scan size of $20 \mu \mathrm{m}$ and 10 force points or force lines were recorded. Each map contained 100 force curves. Dwell times were varied between 0-60 s as within SCFS studies (cf. Chapter 2.6.1.1).

Table 2.8: Overview of parameters used for [A] cantilever calibration and [B] obtaining adhesion forces between the GSLs $\mathrm{GM}_{\mathrm{M}}$ and lyso-LacCer for CPM experiments.

\begin{tabular}{cccccc}
\hline \hline & Setpoint & Velocity & Dwell time & Pause time & $\begin{array}{c}\text { Sample } \\
\text { rate }\end{array}$ \\
\hline \hline A & $1 \mathrm{~V}$ & $1 \mu \mathrm{m} / \mathrm{s}$ & $0 \mathrm{~s}$ & $0 \mathrm{~s}$ & $12,5 \mathrm{kHz}$ \\
B & $0.2 \mathrm{nN}$ & $1 \mu \mathrm{m} / \mathrm{s}$ & $0 \mathrm{~s}-60 \mathrm{~s}$ & $5 \mathrm{~s}$ & $12,5 \mathrm{kHz}$ \\
\hline
\end{tabular}

\section{DATA ANALYSIS}

To determine the maximum adhesion forces between $\mathrm{GM}_{\mathrm{M}}$ and lyso-LacCer in the presence or absence of calcium-ions, the force-distance curves of the retraction process were converted with the ASYLUM RESEARCH software to obtain the corrected height for the cantilever bending (tip-sample separation). Afterwards the data was imported into a home-written MATLAB script ${ }^{3}$ in order to perform the baseline correction and the adjustment of the contact point. To determine the maximum adhesion force, a point close to the maximum force was selected similar to SCFS studies (cf. chapter 2.6.1.1).

\subsection{ATOMIC FORCE MICROSCOPY IMAGING}

For recording a topographical image, the tip of the cantilever is approached to the surface of the sample to a defined deflection. By scanning the sample and adjusting the cantilever height to the defined deflection setpoint, a height image of the sample can be created. The set up and measuring principle is described in chapter 2.5.1.

The most common modes for scanning a sample surface are contact and tapping mode (cf. Figure 2.13). In the latter, the cantilever is excited to oscillate, which happens close to resonance frequency of the cantilever. The oscillation amplitude is then used as the feedback parameter.

\footnotetext{
${ }^{3}$ Kindly provided by Dr. Ingo Mey, Institute of Organic and Biomolecular Chemistry, Georg-August University, Göttingen, Germany.
} 
In contact mode, the absolute cantilever deflection is used as a setpoint and the cantilever does not oscillate. Here, the cantilever is dragged along the surface and high shear forces can act. Disturbing effects due to vibrations and loud noises were reduced by vibrational and acoustic isolation. As relatively soft and fluid lipid membrane samples were examined, tapping mode was the method of choice to analyze the lipid membrane's topography.
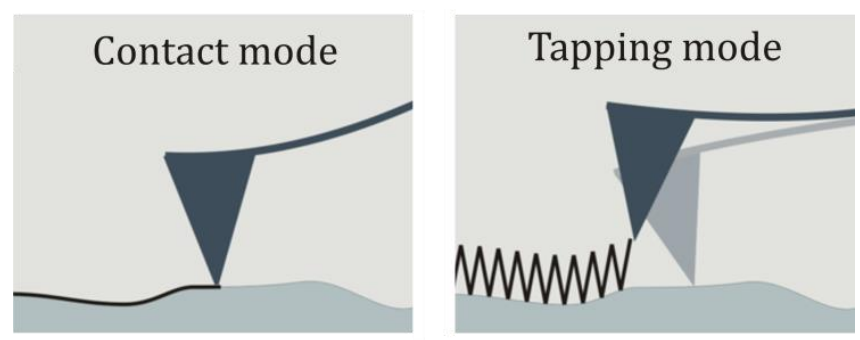

Figure 2.13: Schematic illustration of the two basic measurement principles using imaging atomic force microscopy. In contact mode, the tip stays in the area of repulsive forces, whereas in tapping mode, the cantilever oscillates with a high amplitude. Here, the tip is in the area of repulsive as well as attractive forces during oscillation. ${ }^{129}$

\subsubsection{EXPERIMENTAL PROCEDURE}

AFM imaging was carried out with a MFP-3D Infinity AFM (ASYLUM RESEARCH, Santa Barbara, CA, USA) to examine the surface properties of the GSL containing supported lipid membrane. A type E triangular MSNL-10 cantilever (BRUKER AFM PRoBes, Camarillo, Canada) with a nominal spring constant of $k_{c}=0.1 \mathrm{~N} / \mathrm{m}$ was used. The quality of the fluorescent-labeled lipid membrane on the glass and $\mathrm{SiO}_{2}$ supports were analyzed with an upright BX 51 optical microscope (OLYMPUS, Shinjuku, Japan) using a LUMPLFLN (40x magnification, $N A=0.8$, OLYMPUS, Shinjuku, Japan) objective.

\section{PREPARATION OF SOLID SUPPORTED LIPID MEMBRANES ON $\mathrm{SIO}_{2}$ SUBSTRATES}

Cutting and cleaning of the $\mathrm{SiO}_{2}$ substrates are explained in more detail in Chapter 2.4.3.

To ensure an efficient incorporation of the lipids into the supported lipid membrane, the $\mathrm{SiO}_{2}$ supports as well as custom-made teflon measurement chambers were preheated for about 30 minutes at the highest main phase transition temperature of the used lipids. Subsequently, the solid supports were mounted into the chambers and $200 \mu \mathrm{L}$ of a preheated $1 \mathrm{mg} / \mathrm{mL}$ SUV solution (cf. chapter 2.2.3.1) was pipetted onto the substrates. The solution was incubated for $10 \mathrm{~min}$ before $800 \mu \mathrm{L}$ of degassed $\mathrm{Ca}^{2+}$ spreading buffer (cf. Table 2.2) were added to the SUV solution to maintain a lipid 
concentration of about $0.2 \mathrm{mg} / \mathrm{mL}$. The spreading procedure of SSLMs containing lipids with a main phase transition temperature above or below room temperature are identical to the handling of preparation of SSLM for cell studies (cf. chapter 2.6.1.1). The formed lipid membranes were gently rinsed five times with $1 \mathrm{~mL}$ degassed EDTA- or $\mathrm{Ca}^{2+}$-measurement-buffer (cf. Table 2.2), incubated for 3-4 h as described before, and checked for integrity by an upright BX 51 optical microscope (OLYMPUS, Shinjuku, Japan). Resettled vesicles were removed with respective incubation buffer and directly used for imaging AFM experiments.

All AFM imaging measurements were thankfully performed by DR. HANNES WITT (Max Planck Institute for Dynamics and Self-Organization, Göttingen, Germany). For the topographic analysis of the SSLMs GWYDDION $2.51^{143}$ was used.

\subsection{FLUORESCENCE MICROSCOPY}

To evaluate the success of the formation of SSLMs, the lipid-coupled dyes $\beta$-Bodipy ${ }^{\mathrm{TM}}$ and Texas RedTM DHPE (cf. chapter 2.2.1) were used. With the help of optical, noninvasive fluorescence microscopy, structures and processes on the micrometer scale were visualized.

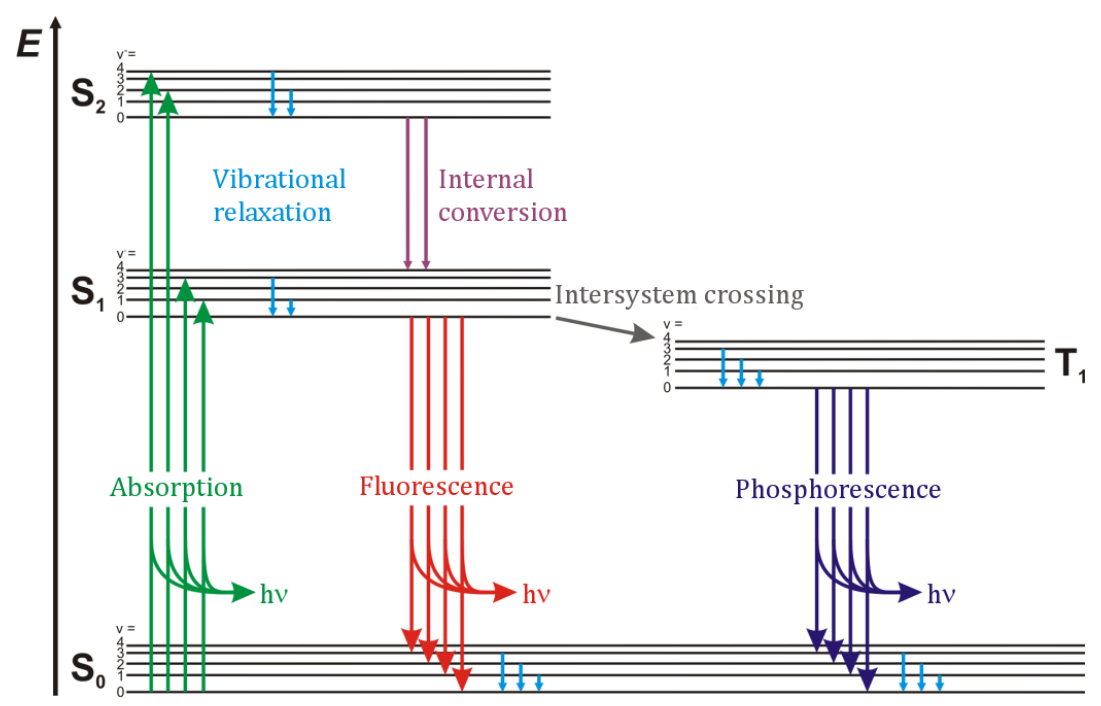

Figure 2.14: Illustration of a Jablonski diagram representing the competing processes between the electronic states $\mathrm{S}_{0}, \mathrm{~S}_{1}, \mathrm{~S}_{2}$ and $\mathrm{T}_{1}$. The respective vibrational quantum numbers $v, v^{\prime}$ and $v^{\prime \prime}$ represent the different vibrational energy levels. 144

In general, fluorescence is the property of certain fluorescent molecules to absorb light of a specific wavelength and release it again with a different wavelength. 
Typically, there is a transition between the highest occupied molecular orbital (HOMO) and the lowest unoccupied molecular orbital (LUMO). The electronically excited singlet state $S_{1}$ is energetically unfavorable and therefore unstable. Consequently, the molecule emits a photon and thus returns to its singlet ground state $S_{0}$ after a few nanoseconds (fluorescence, cf. Figure 2.14). ${ }^{145,146}$ Due to the fact, that the absorbed energy relaxes from an energetically higher vibration state of the electronically excited state into its basic vibration state, the emitted fluorescent light has always a lower energy and is thus shifted to longer wavelengths (StokesShift). ${ }^{145,147}$

\subsubsection{EPIFLUORESCENCE MICROSCOPY}

Epifluorescence microscopy is a widely used method to image fluorescently labeled samples. The schematic drawing of the beam path of an epifluorescence microscope is shown in Figure 2.15 and explained in the following.

At the beginning of the process, the irradiated white light has a continuous spectrum. To selectively filter out the wavelength responsible for the excitation of the molecule, the white light passes through an excitation filter resulting in a monochromatic beam (green). The excitation light is directed to a dichroic mirror, which functions as a beam splitter to reflect shorter and transmit longer wavelengths. This allows the filtered light to reach the sample through an objective and stimulate fluorescence. The emitted fluorescent light (red) in turn passes the dichroic mirror and is then filtered and detected by a camera. The lateral resolution of an epifluorescence microscope is limited by Abbe's law ${ }^{148}$ and is additionally adversely affected by the presence of background fluorescence from fluorescent molecules located in front of or behind the focal plane. This phenomenon causes a degradation of the image quality, which complicates a correct reproduction of three-dimensional structures, such as vesicles or cells. In order to be able to illustrate such objects in a realistic way, confocal laser scanning microscopes are often the method of choice. 


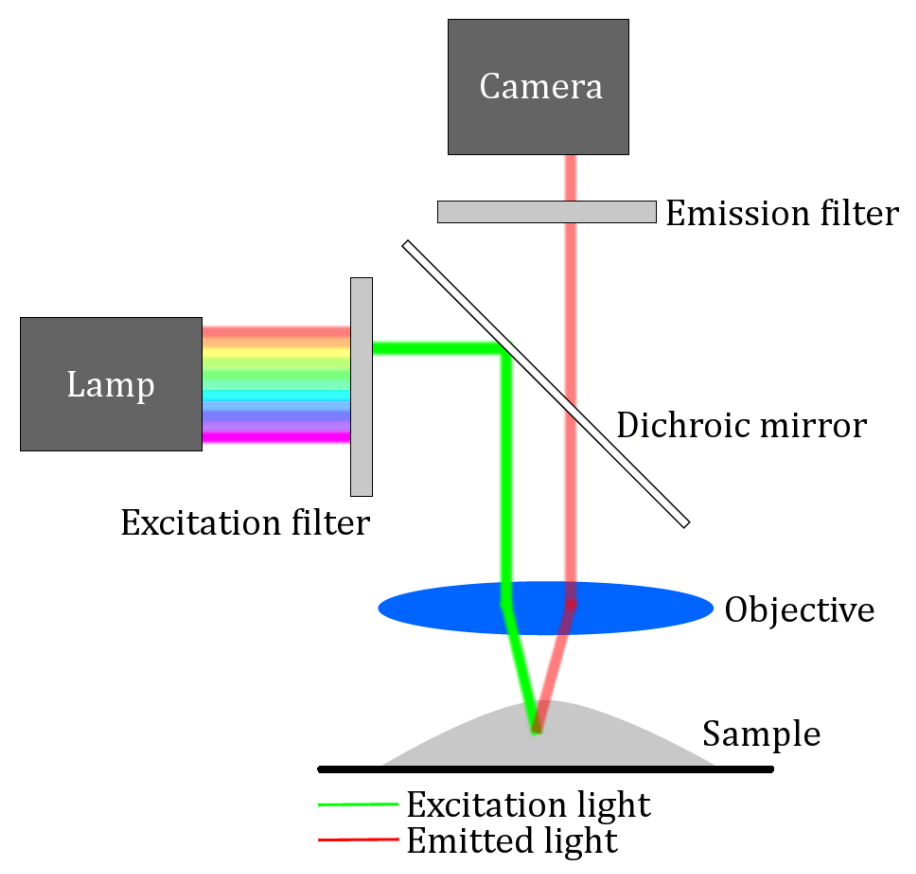

Figure 2.15: Schematic illustration of an epifluorescence microscope. ${ }^{124}$

\subsubsection{EXPERIMENTAL PROCEDURE}

The investigations on the integrity and homogeneity of the fluorescent-labeled solid supported lipid membranes on glass and $\mathrm{SiO}_{2}$ supports were carried out using an upright BX 51 optical microscope (OLYMPUS, Shinjuku, Japan). All images were taken with a water immersion objective (LUMPLFLN 40XW, $N A=0.8$, OLYMPUS, Shinjuku, Japan) and suitable filter cubes (Chroma Technology Corp., Bellows Falls, VT, USA, cf. Table 2.9).

Table 2.9: Overview of filter cube sets (Chroma TEChnology CoRP., Bellows Falls, VT, USA) used within fluorescent microscopy studies.

\begin{tabular}{cccc}
\hline Filter cube set & $\begin{array}{c}\text { Excitation } \\
\lambda_{\text {exc }} / \mathbf{n m}\end{array}$ & $\begin{array}{c}\text { Emission } \\
\boldsymbol{\lambda}_{\text {em }} / \mathbf{n m}\end{array}$ & $\begin{array}{c}\text { Beam splitter } \\
\boldsymbol{\lambda}_{\mathrm{BS}} / \mathbf{n m}\end{array}$ \\
\hline \hline 41001 & $480 \pm 20$ & $535 \pm 25$ & 505 \\
41002 & $535 \pm 25$ & $610 \pm 37.5$ & 565 \\
\hline
\end{tabular}

Editing and analysis of the fluorescence images were performed with the software FIJI. ${ }^{149}$ 


\subsubsection{CONFOCAL LASER SCANNING MICROSCOPY}

The development of conventional fluorescence microscopy to confocal laser scanning microscopy (CLSM) has brought considerable advantages, especially in the field of biology and medicine. ${ }^{150}$ Based on the concept of MINSKY 151 in 1955, an essential difference to the epifluorescence microscope is the scanning process and the subsequent image processing. In an epifluorescence microscope (cf. chapter 2.8.1), all object spots are recorded simultaneously and transferred into an image. In contrast, when using CLSM, the sample is serially irradiated with a spot-by-spot illumination via an oscillating $x-y$ scanning mirror.

In Figure 2.16, a confocal beam path is shown. It can be seen that the excitation light (green) passes a collimator and is focused by a dichroic mirror and an objective in the sample. The resulting emitted light (red) passes the same objective and the dichroic mirror again and is focused on an adjustable confocal pinhole before being detected. By this, stray light from fluorescent molecules above and below the focal plane (dashed grey lines) is inhibited, which reduces the background fluorescence. In order to visualize three-dimensional structures individual layers of the sample are scanned (z-stacks) and reconstructed.

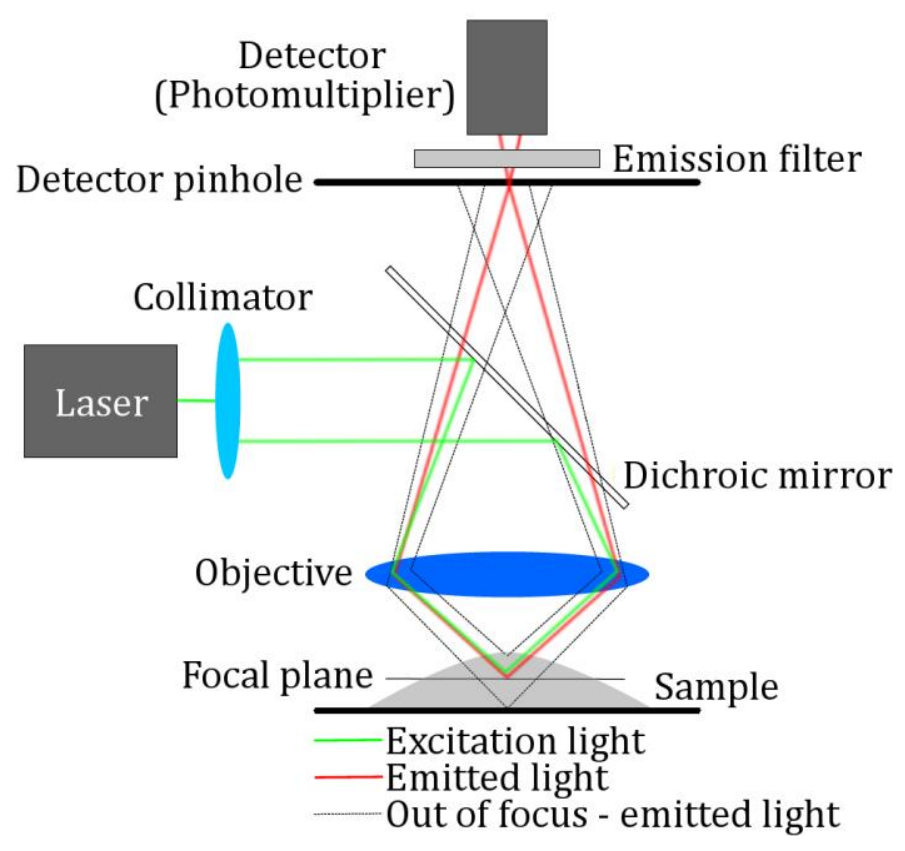

Figure 2.16: Schematic illustration of a confocal scanning laser microscope.124 


\subsubsection{EXPERIMENTAL PROCEDURE}

To visualize the nuclei of the B16-F1 and B16-10 cell line as well as the Gм3 amount on the outer leaflet of the cellular plasma membrane, CLSM studies were carried out using a FluoView 1200 CLSM (OLYMPUS, Shinjuku, Japan). The images were taken with oil immersion objectives with a 60x (UPLFLN60XOIPH, $N A=1.25$, OLYMPUS, Shinjuku, Japan) or 100x magnification (UPLFLN100XO2PH, $N A=1.3$, OLYMPUS, Shinjuku, Japan). Appropriate diode lasers $\left(\lambda_{\text {exc }}=405 \mathrm{~nm}, \lambda_{\text {exc }}=488 \mathrm{~nm}, \lambda_{\text {exc }}=561 \mathrm{~nm}\right)$ ensured the excitation of the fluorescent molecules. Editing and analysis of the images were performed with the FLuoVIEw 1000 software (OlympuS, Shinjuku, Japan), BITPLANE IMARIS (OLYMPUS, Shinjuku, Japan) or FIJI. ${ }^{149}$

\section{B16 CELL LINE FUNCTIONALIZATION}

Both the nuclei staining procedure as well as the labeling of the GSL GM3 of the B16F1 and B16-10 cell lines are performed based on the immunostaining protocols described in chapter 2.1.2.1.

\subsubsection{FLUORESCENCE RECOVERY AFTER PHOTOBLEACHING}

In the 1970's, AXELROD et al. ${ }^{152}$ developed the fluorescence recovery after photobleaching (FRAP) technique, which is a fluorescence-based method that allows the bleaching of fluorescently labeled molecules to investigate dynamic processes with high temporal and spatial resolution. ${ }^{153}$ Together with the fluorescence correlation spectroscopy (FCS) method ${ }^{154}$, which has been developed within the same time frame, it is one of the most important analytical methods for determining diffusion rates of molecules within the plasma membrane of cells or in artificial systems. In this work, FRAP is used to analyze the diffusion of lipids in GSLincorporated SSLMs in order to draw conclusions about the fluidity and the mobile parts of the prepared membranes. For this purpose, fluorescent-labeled molecules are bleached in a certain region of interest (ROI) with a strong laser pulse and are thus irreparably damaged. By passive diffusion of intact fluorescently labeled molecules into the bleached area, the defective molecules are exchanged and a recovery of fluorescence intensity can be observed. The analysis of the time course of the 
fluorescence recovery gives a measure of exchange processes of the lipids in the membrane.

\subsubsection{EXPERIMENTAL PROCEDURE}

In order to analyze the lateral diffusion of the glycosphingolipids lyso-LacCer and Gмз, which were incorporated into glass or $\mathrm{SiO}_{2}$ supported lipid membranes, FRAP studies were performed with a FluoView 1000 CLSM (OLYMPUS, Shinjuku, Japan), equipped with a water immersion objective (LUMFLN 60XW, NA=1.1, OLYMPUS, Shinjuku, Japan) and suitable diode lasers $\left(\lambda_{\text {exc }}=488 \mathrm{~nm}, \lambda_{\text {exc }}=561 \mathrm{~nm}\right)$. Editing and analysis of the images were performed with the FLuoVIEw 1000 software (OLYMPUS, Shinjuku, Japan) or FIJI. ${ }^{149}$

\section{FRAP SETTINGS AND PARAMETERS}

Keeping the continuous bleaching during the FRAP recordings as low as possible, a $5 \mu \mathrm{W}$ excitation laser pulse was used combined with a recording time of $10 \mu \mathrm{s} / \mathrm{pixel}$. To determine the diffusion coefficient of the glycosphingolipid-enriched membrane, a FRAP series of 20 images were taken, of which the first two did not undergo photobleaching (pre bleach). The bleaching process was carried out using a duration of $5 \mathrm{~s}$ and a round ROI using the tornado setting.

\section{DATA ANALYSIS}

In order to determine the diffusion coefficient and the mobile parts of the SSLM, the time course of the fluorescence recovery after photobleaching was analyzed by the following equation 2.2 :

$$
I(t)=I_{\mathrm{eq}}-I_{1} \cdot e^{-t / T_{1}}
$$

Here, $I(t)$ is the fluorescence intensity at the respective time $t, I_{\text {eq }}$ the intensity of the bleached ROI after reaching the equilibrium state and $I_{1}$ the amount of the intensity of the recovered fraction. By applying a mono-exponential fit, the time constant $T_{1}$ can be obtained. From equation 2.3, it is possible to determine the diffusion time $\tau_{\mathrm{D}}$, in which half of the fluorescence intensity after the photobleaching in the selected ROI was reached again.

$$
\tau_{\mathrm{D}}=\ln (0.5) \cdot\left(-T_{1}\right)
$$


By means of the radius $\omega$ of the ROI and the diffusion time $\tau_{\mathrm{D}}$, the characteristic diffusion coefficient $D$ can be calculated following equation 2.4 .

$$
D=\frac{\omega^{2}}{4 \cdot \tau_{\mathrm{D}}}
$$

In general, GSLs are known to form GSL patches in the plasma membrane. For this reason, it is interesting to determine the mobile part $F_{m}$ within the plasma membrane by means of equation 2.5 .

$$
F_{m}=\left(\frac{I_{1}}{I_{\mathrm{i}}-I_{0}}\right)
$$

Here, $I_{\mathrm{i}}$ corresponds to the fluorescence intensity before bleaching and $I_{0}$ to the fluorescence intensity after bleaching.

\subsubsection{TOTAL INTERNAL REFLECTION FLUORESCENCE}

The adhesion of cells in multicellular organisms is an essential prerequisite for the formation of tissue and organs. Large numbers of adhesion molecules in the plasma membrane of cells control the attachment process. However, these near-surface molecules are usually poorly visualized by classical fluorescence microscopy, since the near-surface signal is obscured by the background scattered light. In 1956, AMBROSE $^{155}$ described for the first time a way to visualize fluorescent molecules on the interface between the cell surface and the glass substrate by means of the total internal reflection fluorescence (TIRF). Over the years, this technique has become an important method for determining the localization and dynamics of cell membrane molecules.

The basis of this technique is Snell's law of refraction (cf. equation 2.6), which describes the bending of light when there is a change in refractive index passing from one material into another.

$$
n_{1} \cdot \sin \left(\theta_{1}\right)=n_{2} \cdot \sin \left(\theta_{2}\right)
$$

Here, $n_{1}$ and $n_{2}$ are the refractive indices of the differentiable media, $\theta_{1}$ and $\theta_{2}$ are the angle of incidence and the angle of refraction, respectively. In Figure 2.17 the difference between epifluorescence and TIRF microscopy are shown. If the beam of light (blue arrows) passes e.g. through glass and water, which has a lower refraction index compared to glass, the light will bend to a larger angle from normal. If the critical 
angle $\theta_{\mathrm{C}}$ (dotted line) is exceeded, the total internal reflection is achieved and the light is reflected (red arrow) and will not enter the second media or sample. At this point, an evanescent wave is generated, which decays exponentially and reaches about 100$200 \mathrm{~nm}$ into the second medium and can excite fluorescent-labeled molecules (green dots) near the interface.

[A] Epifluorescence
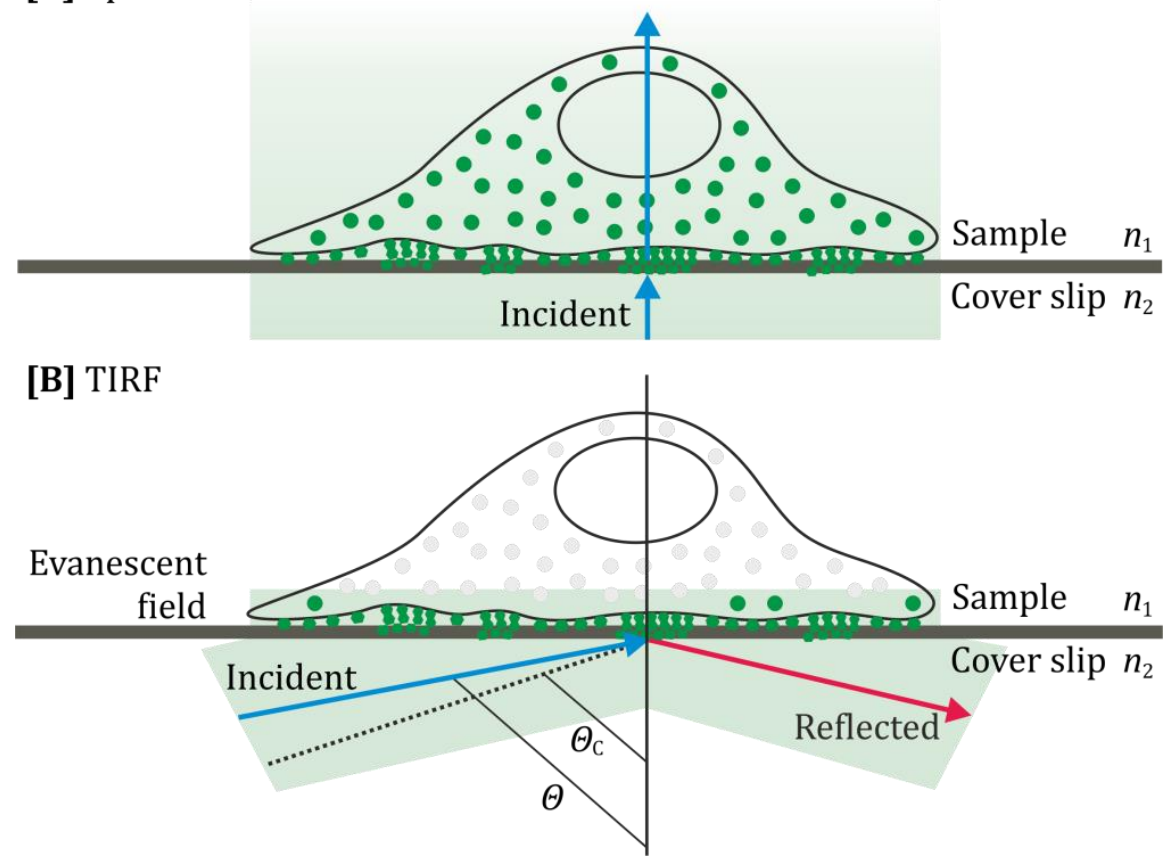

Figure 2.17: Schematic illustration of the physical basis of epifluorescence and TIRF microscopy. In general, fluorescence molecules (green dots) within the sample are excited by an appropriate incident light (blue arrows). Whereas in the case of epifluorescence [A] each molecule is excited to fluorescence due to light passing directly through the interface between cover slip and sample, within TIRF [B], the light hits the cover slip at a certain incidence angle $\Theta$. Because this angle is larger than the critical angle $\Theta_{c}$ (dotted line), the incident light is reflected at the cover slip (red arrow). The resulting evanescence field protrudes only a few hundred nanometers into the sample, thus stimulating only those molecules that are within the field.156

\subsubsection{EXPERIMENTAL PROCEDURE}

Within TIRF experiments, special attention was paid to the distribution of $\mathrm{G}_{\mathrm{M} 3}$ within the cellular plasma membrane of the B16 melanoma cancer cells. All total internal reflection fluorescence images were carried out with an inverted IX 81 optical microscope (OLYMPUS, Shinjuku, Japan), which was combined with a cellTIRF (OlYMPUS, Shinjuku, Japan) unit. The images were obtained using an oil immersion objective (PLAPOOTIRFM, NA=1.45, OlYMPUS, Shinjuku, Japan) with a $60 \mathrm{x}$ magnification and a suitable diode laser $\left(\lambda_{\mathrm{exc}}=488 \mathrm{~nm}\right)$. Editing and analysis of the images were performed with the CELLSENS DIMENSION software (V1.15, OLYMPUS, Shinjuku, Japan) and FIJI. ${ }^{149}$ 


\section{B16 CELL LINE FUNCTIONALIZATION}

The labeling of the GSL GM3, which is embedded into the outer leaflet of the B16-F1 and B16-10 cell lines are performed according to the immunostaining protocols described in chapter 2.1.2.1. For TIRF studies, approximately 50,000 cells were transferred to $2 \mathrm{~mL}$ of warm $\mathrm{D} 10 \mathrm{~F}^{-}$medium and grown for $24 \mathrm{~h}$ in $35 \mathrm{~mm}$ petri dishes with 14 mm glass microwells (No. 1.5, MatTek Corporation, Ashland, MA, USA). The glass petri dishes were washed with ethanol p.a. and D10F- medium beforehand to ensure a clean and cell-friendly surface. The stained and fixed cells were directly used for TIRF measurements.

\subsubsection{IMAGE-BASED CYTOMETRY}

In this thesis, image-based cytometry studies were performed to determine the amount of the GSL GM3 on the extracellular plasma membrane of the B16-F1 and B16F10 cells. In contrast to flow cytometry, which was developed by Wolfgang Göhde in 1968, the image-based variant analyzes the sample on solid supports, while in flow cytometry cells are scanned in suspension. Cytometry in general is a widely used method in today's diagnostic medicine. As a high-throughput screening system, it is suitable for examining cells for their properties, such as proliferation, differentiation or cell-cycle abnormalities. Within flow cytometry, suspended cells passes one by one a light source, usually in the form of a laser beam. In the case of fluorescence labeling, the fluorescent molecules are excited by the light beam to emit fluorescence. The detector can be used to analyze the forward scattered (FSC, forward scatter) as well as the side scattered light (SSC, side scatter). The light emitted by the cell sample is finally converted into an electrical signal in the electrical system by the installation of photomultipliers. The image-based cytometry likewise allows the cell-by-cell analysis of fluorescence intensities by fluorescently labeled cell components but does not rely on diodes for the forward scattered and side scattered light. Additionally, less amounts of the cell samples are necessary and the sample can be rescanned. Thus, comparable results can be obtained to flow cytometry. ${ }^{157}$ 


\subsubsection{EXPERIMENTAL PROCEDURE}

All image-based cytometry measurements were thankfully performed by DR. TABEA OsWALD (Institute of Organic and Biomolecular Chemistry, Georg-August University, Göttingen, Germany).

The experiments were carried out with a Countstar ${ }^{\circledR}$ Rigel S5 advanced image cytometer (InTAS SCIENCE IMAGING InSTRUMENTS GMBH, Göttingen, Germany) to analyze the amount of $\mathrm{G}_{\mathrm{M} 3}$ on the outer surface of the plasma membrane of the B16 cells by applying an antibody-based immunostaining assay (see below). The median fluorescence intensities of each cell line were determined with the FCS Express 6 (DE Novo SofTwARE, Glendale, CA, USA) software and plotted using Origin ${ }^{\circledR}$ (OriginPro 8.5G, Origin Lab Corporation, Northampton, MA, USA).

\section{B16 CELL LINE IMMUNOSTAINING PROTOCOL}

First, the cells were detached from the bottom of the cell culture bottle as described in Chapter 2.1.1. Subsequently, the cells were taken up in $1 \mathrm{~mL}$ of a warm PBS-solution (cf. Table 2.1) and counted. Approximately 1,000,000 cells were transferred to $1 \mathrm{~mL}$ of a warm PBS-- solution and centrifuged (Heraeus Fresco 21, THERMo FiSHER SCIENTIFIC, Waltham, MA, USA) at $4{ }^{\circ} \mathrm{C}$ and $0.3 \mathrm{xg}$ for $3 \mathrm{~min}$ in a micro test tube $(1.5 \mathrm{~mL}$, EPPENDORF, Hamburg, Germany). The supernatant was discarded and the resulting pellet carefully resuspended in $1 \mathrm{~mL}$ of a triton-free blocking buffer (cf. Table 2.1). The cell suspension was incubated for $30 \mathrm{~min}$ on ice and centrifuged $(0.3 \times \mathrm{g}, 3 \mathrm{~min}$, $4{ }^{\circ} \mathrm{C}$ ). Subsequently, the pellet was resuspended in $500 \mu \mathrm{L}$ of a PBS-- solution and centrifuged again $\left(0.3 \times \mathrm{g}, 3 \mathrm{~min}, 4^{\circ} \mathrm{C}\right)$. Again, the supernatant was discarded and the pellet carefully resuspended in $100 \mu \mathrm{L}$ triton-free dilution buffer (cf. Table 2.1) containing $10 \mu \mathrm{g} / \mathrm{mL}$ anti-GM3 monoclonal IgM antibody. After an incubation period of $1 \mathrm{~h}$ at $4{ }^{\circ} \mathrm{C}$, the solution was washed three times by centrifugation $(0.3 \times \mathrm{g}, 3 \mathrm{~min}$, $4{ }^{\circ} \mathrm{C}$ ) and resuspension in $1 \mathrm{~mL}$ of PBS--. Then, the obtained pellet was carefully resuspended in $100 \mu \mathrm{L}$ of a triton-free dilution buffer containing $5 \mu \mathrm{g} / \mathrm{mL}$ goat antimouse IgG/IgM secondary antibody conjugated with Alexa Fluor 488. After an incubation period of $1 \mathrm{~h}$ at $4{ }^{\circ} \mathrm{C}$, the solution was washed 3 times with $1 \mathrm{~mL}$ of PBS-as described above. Finally, the pellet was resuspended in $100 \mu \mathrm{L}$ of PBS--. For the subsequent measurements, $20 \mu \mathrm{L}$ of the cell solution was added to a CoUNTSTAR 
ChAmber Slide and analyzed using the GFP (488) PI BioApP (both INTAS SCIENCE IMAGing InSTRUMENTS GMBH, Göttingen, Germany). Data analysis was performed using the FCS EXPRESS 6 software (DENovo, Glendale, CA, USA).

For control measurements, all immunostaining steps were identical to the abovementioned process with the difference that either no (blank sample) or only the secondary antibody (negative control) was used.

\subsubsection{CELL ADHESION ASSAY}

Proper cell adhesion is indispensable to maintain vital processes in an organism. Within this work, the cell adhesion assay should provide insights into the adhesive properties of the Gм3-expressing B16-F1 and B16-F10 cells on lyso-LacCer-doted SSLMs.

\subsubsection{EXPERIMENTAL PROCEDURE}

All cell adhesion studies were performed using an inverted IX 83 optical microscope (OLYMPUS, Shinjuku, Japan), which was combined with a heating (temperature controller, IBIDI GMBH, Martinsried, Germany) and incubation (gas mixer, IBIDI GMBH, Martinsried, Germany) system. The images were obtained using an UPLFLN 10X2 objective ( $N A=0.3$, OLYMPUS, Shinjuku, Japan) with a $10 \mathrm{x}$ magnification and a suitable diode laser $\left(\lambda_{\text {exc }}=488 \mathrm{~nm}\right)$. All experiments were performed at $7.5 \% \mathrm{CO}_{2}$ saturation and $37^{\circ} \mathrm{C}$. By staining the cell nucleus with Hoechst 33342 (cf. chapter 2.1.2.1), it was possible to track the center of the nucleus. About 20.000 cells in 3 mL PBS-- were seeded onto fibronectin-coated glass substrates or POPC/lyso-LacCer/Bodipy (89:10:1 mol\%)-doped SSLMs and immediately stained with Hoechst 33342 (1:10,000). For fibronectin-coating, $35 \mathrm{~mm}$ glass bottom petri dishes with a $14 \mathrm{~mm}$ glass microwell (No. 1.5, MATTEK CoRporation, Ashland, MA, USA) were coated with $5 \mu \mathrm{g} / \mathrm{cm}^{2}$ fibronectin (human plasma, Sigma ALDRICH, St. Louis, MO, USA) and incubated at room temperature for $1 \mathrm{~h}$. Excess fibronectin-coating was removed and used immediately. The preparation of lyso-LacCer-doted SSLMs is described in Chapter 2.6.1.1. 


\subsection{REFLECTION INTERFERENCE CONTRAST MICROSCOPY}

With the establishment of the total internal reflection fluorescence (TIRF) technique, an important tool has been developed to precisely determine near-surface processes such as cell adhesion. A disadvantage of this method, however, is that the molecules which have to be labeled with fluorophores for visualization, can be influenced in their function. By contrast, the reflection interference contrast microscopy (RICM) technique, is a label-free method and thus rules out disturbing effects of fluorescent modified cell components. Therefore, this non-invasive method has the great advantage of a live optically tracking of the adhesion points of cells at different surfaces. ${ }^{158,159}$ Still, RICM shares many of the advantages of TIRF microscopy over conventional bright field microscopy: high contrast, high definition and enhanced sensitivity. Studying the movements of living cells was one of the first applications for RICM used by ABERCROMBIE and AMrose ${ }^{160}$ in 1954 and CurTis ${ }^{161}$ in 1964. In 1975, PloEM ${ }^{162}$ succeeded in using the antiflex technology to improve the process again.

In general, RICM makes use of polarized light to detect interferences within the sample, which then provide insights into the cell-substrate distance. ${ }^{160,162}$ Both beams of light differ in their optical path lengths in their way through the media generating a constructive or destructive interference. A detector bundles this information and displays the interference pattern of the sample. From the obtained interference patterns, the height image of the object on the substrate can be determined. The pattern allows a penetration depth of around $200 \mathrm{~nm}$ in the sample achieving a deeper insight into the cell-substrate distance. Due to the influence of different refractive indices and multiple reflections of the objects and substrates, it is challenging to carry out correct calculations of the cell-substrate distance ${ }^{163,164}$. However, since the distances of the cell components to the substrate can be reliably differentiated from the determination of the color intensities within a RICM image, an exact analysis of the distance was omitted and the focus was placed on the classification of the color regions within the recorded images. Due to the different interferences of the reflected light, it is possible to draw conclusions about close or further adhesion points of the cells from the surface. ${ }^{165} \mathrm{~A}$ measure of the distance of an object to a surface can be obtained from the different gray coloration of the RICM image. Thus, IzZARD et al. ${ }^{165}$ classified the different shades of gray into dark gray to 
black objects, which represent very close distances of $15-30 \mathrm{~nm}$. White areas, on the other hand, reflect objects that have the greatest distance to the surface at a distance of $100 \mathrm{~nm}$.

\subsubsection{EXPERIMENTAL PROCEDURE}

All RICM studies were performed using an inverted Axiovert 200 optical microscope (ZEISS, Oberkochen, Germany), which was equipped with an oil immersion PlanNeofluar antiflex objective (63x/1.25, Ph3, a = 0.09 mm, ZEISS, Oberkochen, Germany). Beyond, a polarization module (Pol ACR P\&C for HBO 10, ZEISS, Oberkochen, Germany), which was suitable for high-energy illumination, for the incident white light (X-Cite(R) 120Q, OLYMPUS, Shinjuku, Japan) was used. The polarized light passes the antiflex objective equipped with a $\lambda / 4$ wave plate to reach the sample. Here, the light beam is partially reflected and passes an analyzer before an image is formed. An additional integrated UV filter blocks the UV emission of the light source to protect cells and the eye of the observation from UV radiation.

For RICM studies, approximately 50,000 cells were transferred to $2 \mathrm{~mL}$ of warm D10F- medium and grown for $24 \mathrm{~h}$ in pure or fibronectin coated (cf. Chapter 2.8.6.1) $35 \mathrm{~mm}$ petri dishes with $14 \mathrm{~mm}$ glass microwell (No. 1.5, MATTEK CORPORATION, Ashland, MA, USA). By using a heating (temperature controller, IBIDI GMBH, Martinsried, Germany) and incubation (gas mixer, IBIDI GMBH, Martinsried, Germany) system, all experiments were performed at $7.5 \% \mathrm{CO}_{2}$ saturation and $37^{\circ} \mathrm{C}$. The glass petri dishes were washed with ethanol p.a. and D10F- medium beforehand to ensure a clean and cell-friendly surface. Editing and analysis of the images were performed with FIJI. ${ }^{149}$ 



\section{RESULTS AND DISCUSSION}

The main goal of this thesis was to investigate the impact of carbohydratecarbohydrate interactions (CCIs) on the adhesion of $\mathrm{G}_{\mathrm{M} 3}$-containing murine B16 melanoma cancer cells. For this purpose, the calcium-dependent interaction between the globoside lyso-lactosylceramide (lyso-LacCer) and the ganglioside $\mathrm{G}_{\mathrm{M}}$ was examined based on model membranes and cell studies. The amount of $\mathrm{G}_{\mathrm{M} 3}$ in the cellular plasma membrane of B16-F1 and B16-F10 cells was examined and compared to the adhesive properties of the cells related to their malignancy. To assess the binding strength between $\mathrm{G}_{\mathrm{M} 3}$ and lyso-lactosylceramide single-cell force spectroscopy (SCFS) and colloidal probe microscopy (CPM) studies based on atomic force microscopy (AFM) were performed.

\section{1 $\mathrm{G}_{\mathrm{M} 3}$ EXPRESSION OF THE B16 CELL LINE}

Within this project, the GM3-overexpressing murine B16 melanoma carcinoma cell lines B16-F1 and B16-F10 are used as a model system for cancer cell lines with a different malignancy. ${ }^{166,167}$ B16 cells were first isolated by FIDLER ${ }^{5,6}$ revealing a high degree of metastasis for the B16-F10 cell line contrary to the F1 variant having a rather low tendency for metastasis. ${ }^{6}$

Firstly, the distribution of the glycosphingolipid (GSL) GM3 within the cellular plasma membrane was analyzed by confocal laser scanning microscopy (CLSM). By recording $x-z$ and $y-z$ planes, the three-dimensional structure of cells can be visualized and used to determine those cell regions, in which the gangliosides 
accumulate primarily. In Figure 3.1, the sub-confluent grown [A] B16-F1 and [B] B16F10 cell layers are shown, labeled for the ganglioside $\mathrm{G}_{\mathrm{M} 3}(\square)$ and the cellular nuclei (घ) according to the immunostaining procedure described in chapter 2.1.2.1. Since the glycosphingolipids are incorporated into the outer leaflet of the cell membrane ${ }^{41}$, a triton-free blocking and dilution buffer was used (cf. Table 2.1, Chapter 2.1) to keep the membrane as intact as possible. Detergents like Triton X-100 are used to lyse the cell or to permeabilize the cellular plasma membrane ${ }^{168-170}$ to stain specific components within the cell. When looking at the confocal images in Figure 3.1, it was noticeable that $\mathrm{G}_{\mathrm{M} 3}$ seemed to be punctually embedded into the membrane rather than being homogenously distributed. These findings were evident in both the $[\mathrm{A}]$ B16-F1 and [B] the B16-F10 cell line and agreed well with the assumption that GSLs in general form domains within the cellular membrane to initiate the adhesion between two adjacent cells. ${ }^{32,45}$ Besides, the $x-z$ and $y-z$ planes of the confocal images indicated that the ganglioside $\mathrm{G}_{\mathrm{M} 3}$ is predominantly expressed in the outer plasma membrane of the cells, particularly on the apical side, since there are hardly any fluorescent molecules on the basal side of the cell noticeable.
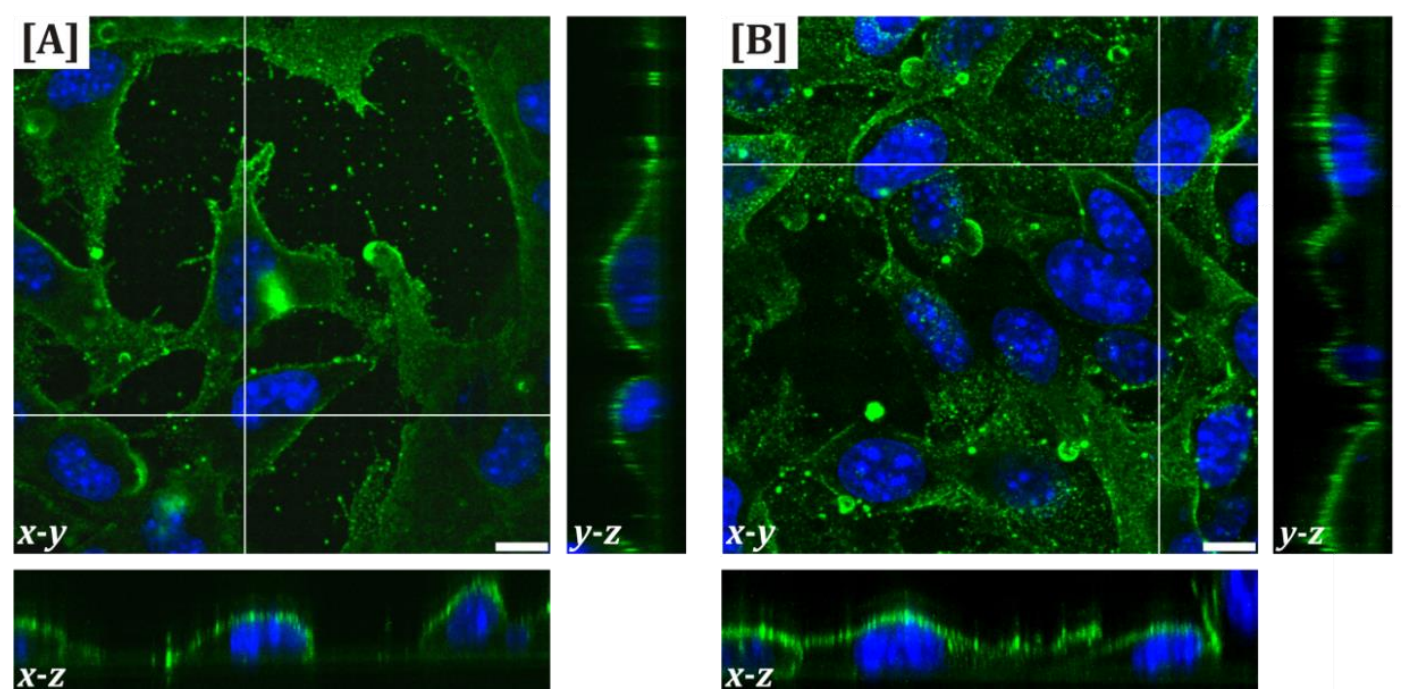

Figure 3.1: Confocal laser scanning microscopy images of sub-confluent grown B16-F1 [A] and B16-F10 [B] cell layers for $48 \mathrm{~h}$. $X-y$ as well as $x-z$ and $y-z$ images visualize the distribution of the ganglioside $\mathrm{G}_{\mathrm{M} 3}(\square)$ within the cellular plasma membranes. The respective cellular nuclei are shown in blue (匹). Cell seeding density: 200,000 cells. Scale bars: $10 \mu \mathrm{m}$.

Since near-surface signals are often covered by background scattered light, CLSM reaches its limits when visualizing molecules close to the surface. Therefore, total internal reflection fluorescence (TIRF) was used to further study the distribution of GM3 on the basal cellular membrane. TIRF is able to visualize structures that are 
about $100 \mathrm{~nm}^{156,171}$ away from the surface and is helpful to uncover selective processes in the basal cellular plasma membrane. Due to this, the method has become an important tool to detect and explore adhesion relevant processes like the dynamic of $\operatorname{actin}^{172,173}$ or the orientation of microtubules to focal adhesions ${ }^{174}$. Contrary to the confocal images (cf. Figure 3.1), Figure 3.2 showed that $\mathrm{G}_{\mathrm{M} 3}$ tends to be embedded on the basal side of the cellular membrane indicating that $\mathrm{G}_{\mathrm{M} 3}$ might be recruited to substrate facing regions to enhance adhesion. In addition, it seemed like GM3 was expressed at the cell rims rather than in the center of the cell. Based on this finding, the questions aroused whether $\mathrm{G}_{\mathrm{M} 3}$ is accumulated at adhesion complexes mainly located at the cell periphery ${ }^{175}$ or if the upper and lower cellular membranes were only getting very close to each during cell attachment leading to an increased fluorescence intensity. Another possibility would be an imprecise staining of Gм3 with the used anti-GM3 monoclonal IgM antibody. Even though the latter options are plausible, studies from OKADA et al. ${ }^{26}$ indicated the enrichment of GM3 to focal adhesions or at least to adhesive complexes at the attachment sites of the cell. Within the study, baby hamster kidney (BHK-C12) cells were treated with EDTA and the zwitterionic detergent Empigen BB to collect the molecules that are targeted to the attachment sites of the cell. The analysis revealed the enrichment of GSLs, especially of $\mathrm{G}_{\mathrm{M} 3}$, to those attachment sites.
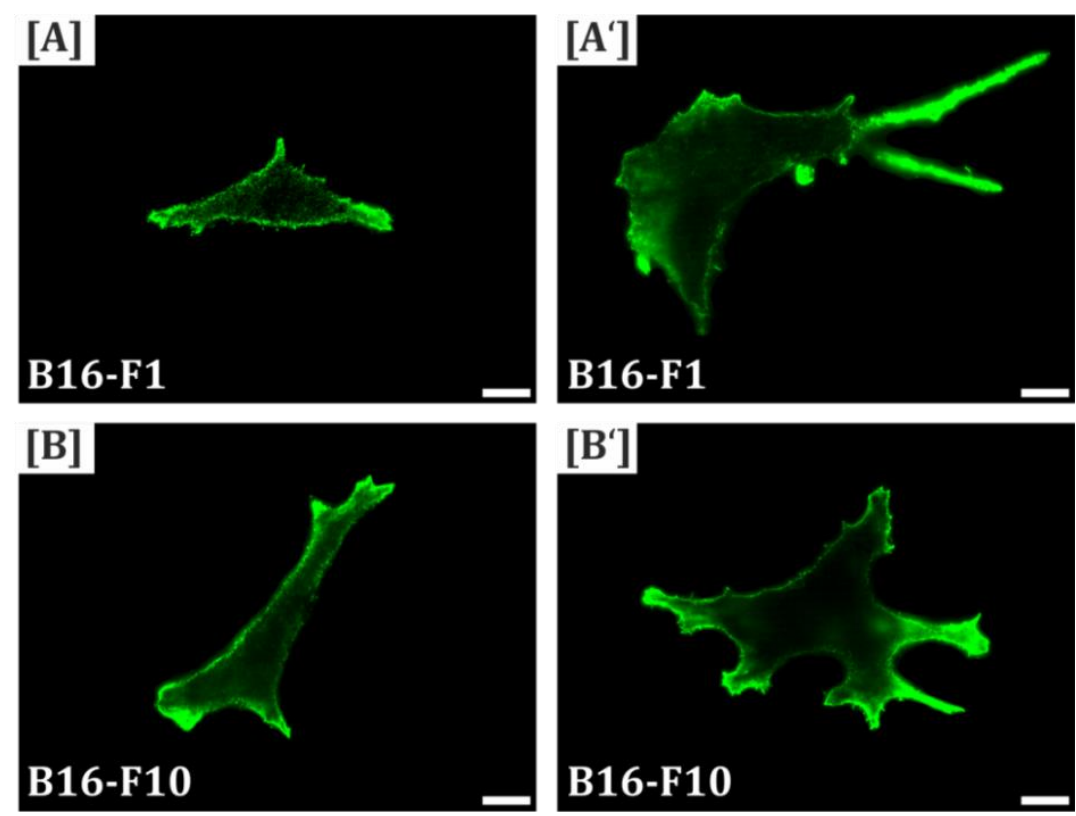

Figure 3.2 Total internal reflection fluorescence images of typically shaped single B16-F1 [A, A'] and B16-F10 [B, $\left.\mathrm{B}^{\prime}\right]$ cells grown on glass surfaces for $24 \mathrm{~h}$, respectively. The images show that the ganglioside $\mathrm{G}_{\mathrm{M} 3}(\square)$ is mainly distributed at the cell rims and present at the basal side of the membrane. Cell seeding density: 50,000 cells per petri dish. Scale bars: $10 \mu \mathrm{m}$. 
Evidence exists that the more metastatic cancer cell line B16-F10 expresses a higher level of $\mathrm{G}_{\mathrm{M} 3}$ than the B16-F1 variant. 67,85,86,176 This correlation was examined mostly within studies of HAKOMORI and coworkers ${ }^{85,86}$. By means of fluorescence-activated cytometry experiments, the amount of Gм3 expressed on the surfaces of B16-F1 and B16-F10 was detected by incubating the cells with an anti-GM3 monoclonal DH2 (IgG3) antibody, which in turn was coupled to a fluorescein-labeled goat anti-mouse IgG antibody 85,86 . The cytometry measurements revealed a slightly higher amount of $\mathrm{G}_{\mathrm{M} 3}$ expressed on the surface of the highly metastatic cancer cell line B16-F10 in contrast to the F1 variant and thereby indicated a correlation of the $\mathrm{G}_{\mathrm{M} 3}$ expression to the metastatic potential of the B16 mouse melanoma cell lines. Studies from SAWADA et al., ${ }^{176}$ using a primary anti-GM3 IgM antibody, confirm the results.

Within this project, image-based cytometry was applied to examine the amount of GM3 on the plasma membrane of the B16-F1 and B16-F10 cells. With the help of this method, the correlation of the cell size and the integrated fluorescence intensity of GM3 were visualized as demonstrated in Figure 3.3 [A].

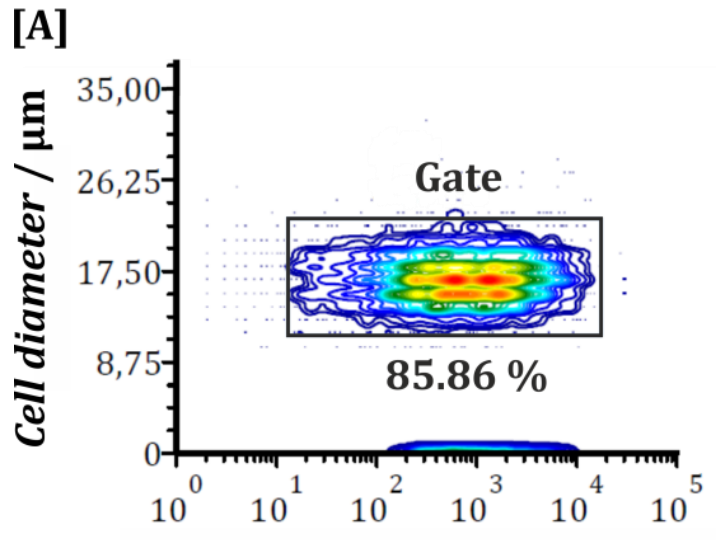

Integrated fluorescence intensity
[B]

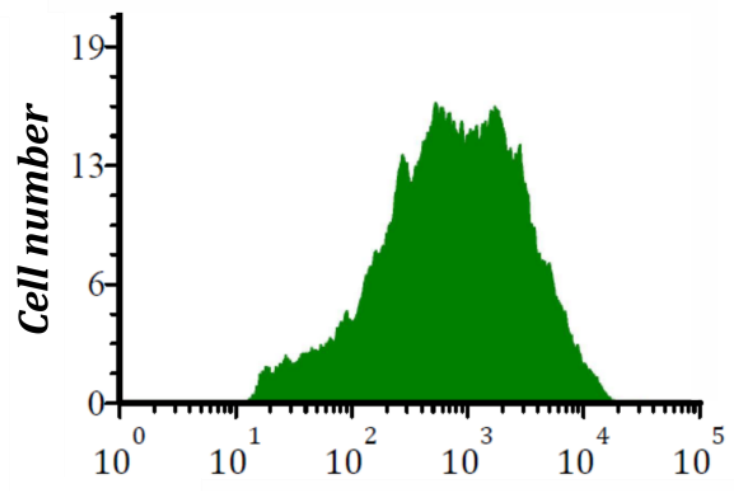

Integrated fluorescence intensity

Figure 3.3: Exemplary image based cytometry results to investigate the total amount of $\mathrm{G}_{\mathrm{M} 3}$ expressed on the surface of the B16-F1 cells. In [A] the cells are characterized by their size and integrated fluorescence intensity. A gate was set to further analyze only the fluorescence intensity of vital cells. Every dot indicates one count, red color a high amount of counts and blue a low amount of counts. In [B] the selected data from [A] is displayed in a histogram (green), showing the fluorescence intensity distribution of $\mathrm{G}_{\mathrm{M}}$ expressing cells.

A specific gate (dark gray frame) was selected to ensure that only those cells are chosen, which have a good vitality and thus show an appropriate fluorescence intensity. Every dot indicated one count with red dots representing a high amount of counts and blue dots illustrating a low amount of counts. In the example given in Figure 3.3, about $86 \%$ of the total amount of the B16-F1 cells was selected for further analysis. Signals having a cell diameter of less than $10 \mu \mathrm{m}$ represented dead cells or 
residues of cell components and were therefore neglected. Afterwards, the selected cell data was visualized in a histogram (cf. Figure 3.3 [B]). For the examination of the overall amount of $\mathrm{G}_{\mathrm{M} 3}$ expressed on either the B16-F1 and the B16-F10 surfaces, the data was selected in a way that about 50-86\% of the total amount of cells was analyzed.

To compare all performed experiments, the median fluorescence intensities of the B16-F1 and B16-F10 cells are displayed in Figure 3.4. For control measurements, either no (blank sample) or only the secondary goat anti-mouse antibody (cf. Chapter 2.1.2.1, negative control) was used. All control studies revealed no fluorescence intensities at all, proving that the anti- $\mathrm{G}_{\mathrm{M} 3}$ monoclonal IgM antibody is very specific and can be selectively used to label the glycosphingolipid Gмз. Based on these findings, all control values were collected, averaged up to one value (black rhombus) and plotted as "control" (cf. Figure 3.4, dotted green or red line).

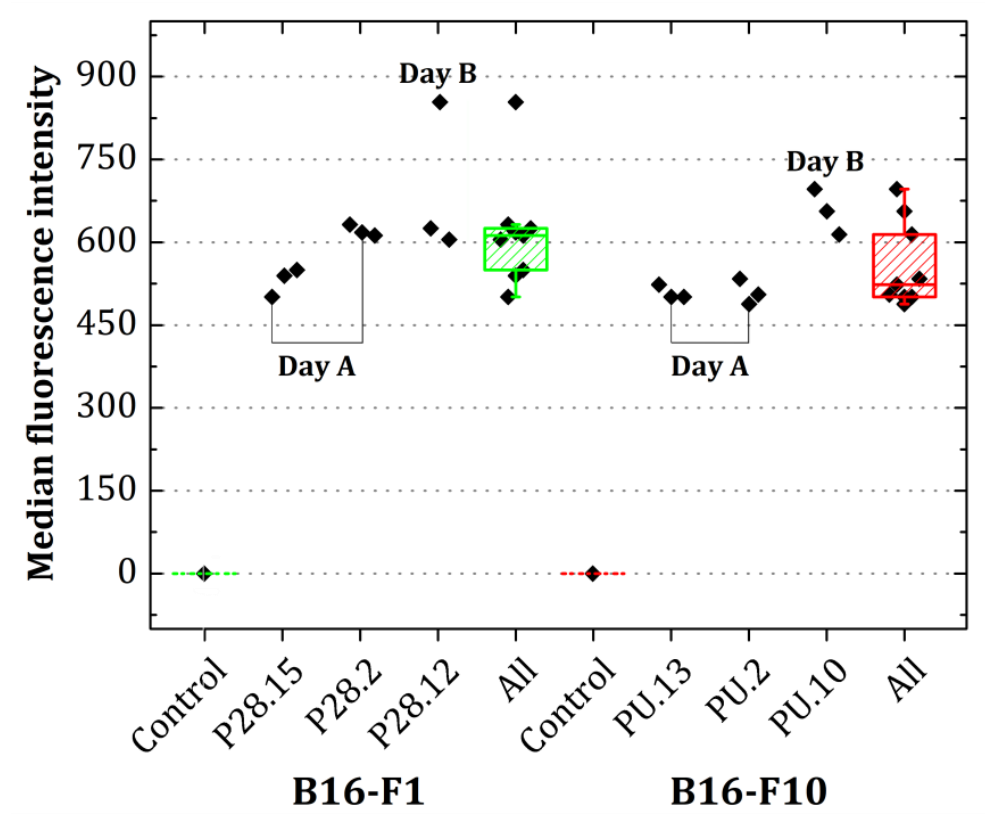

Figure 3.4: Fluorescence-based cytometry analysis of the $\mathrm{G}_{\mathrm{M} 3}$ amount, expressed on the surface of the B16-F1 (green) and B16-F10 (red) carcinoma cell line. The black rhombs show the obtained fluorescence intensities of the antibody-labeled $\mathrm{G}_{\mathrm{M} 3}$ within different passages of B16-F1 and B16-F10 on day A and B. Control measurements are illustrated via a dotted line and all experimental results combined are shown in a box plot. Here, $50 \%$ of all data points are represented by a box containing the median (solid line). The box is limited by an upper (75\% threshold) and lower (25\% threshold) quartile. Data points outside of the box, whose values are limited to 1.5 times the box length ${ }^{177}$, are described by whiskers. Values, which are not within the whisker range, are referred to as outliers.

In the present study, P28.2, P28.12, P28.15 as well as PU.2, PU.10 and PU.13 represent the different passage numbers of the B16-F1 and B16-F10 cell lines, respectively, which were used within different days A and B. Here, P28.12 and P28.15 originates 
from the same subculture, whereas P28.2 was freshly subcultured and jointly used with P28.15 for comparable cytometry experiments on day A. The same applies to the B16-F10 cell line. For a final comparison, all fluorescence intensities, that were obtained within the passage numbers of the respective cell lines, were combined and represented in a box plot (cf. Figure 3.4 "All”).

Although an increased $\mathrm{G}_{\mathrm{M} 3}$ expression on the surface of the strongly metastasizing cancer cell line B16-F10 was demonstrated in several studies $85,86,176$, our cytometry studies did not show that the more metastatic B16-F10 cell line has a higher proportion of $\mathrm{Gm}_{\mathrm{M} 3}$ expressed on its surface. Even though intensities varied over day and cell sample, the median of all collected intensities $\left(\widetilde{F}_{\mathrm{F} 1}=612\right.$ for the B16-F1 and $\tilde{F}_{\mathrm{F} 10}=523$ for the B16-F10 cell line) show a slightly larger value for the F1 variant. Based on these data, we cannot conclude that the B16-F10 cell line shows a highly increased $\mathrm{G}_{\mathrm{M} 3}$ expression compared to the B16-F1 variant.

Looking more closely at the fluorescence intensities of the individual experiments, it is noticeable that the measured amount of $\mathrm{G}_{\mathrm{M} 3}$ depends strongly on the day, on which the experiment was carried out rather than on the individual passage number of the cell (e.g. B16-F10, cf. Figure 3.4). These findings coincide with studies revealing that the B16 cells exhibit stable metastatic phenotypes even after constant cell passage ${ }^{5-7}$ at least in uncloned cell lines. ${ }^{8}$ Experiments on day B demonstrated larger median fluorescence intensity values of both the B16-F1 $\left(\tilde{F}_{\mathrm{P} 28.12}=625\right)$ and B16-F10 $\left(\tilde{F}_{\text {PU.10 }}=656\right)$ cell line compared to day A. This suggests that the integrity of cells and their functionality can already be influenced by a slight change in the environment or in day-dependent preparation steps. Thus, cells react sensitively to nutrient deficiency in the medium by a longer growth phase, to a prolonged trypsin incubation time during the harvesting process or simply alter their functionality by building up more ( $\sim$ confluent phase) or less ( $\sim$ sub-confluent phase) contacts with neighboring cells. Especially the effect of the latter was discussed within studies of BoSMANN ${ }^{7}$, who found out that sparse and confluently grown B16-F1 and B16-F10 cells showed significant differences in their surface properties. By comparing the stability of the metastatic potential in cloned and uncloned B16 cell lines, PosTE et al. ${ }^{8}$ found that the uncloned B16-F1 and B16-F10 cell lines are comprised of cell subpopulations holding various phenotypic properties like metastatic potentials. This finding is in line with 
the broad distribution of fluorescence intensities found within our fluorescencebased cytometry analysis of the $\mathrm{G}_{\mathrm{M} 3}$-expression on B16 melanoma cells (cf. Figure 3.3 [B]). In addition, research by KојІмA and HAKOMORI ${ }^{86}$ supported our outcomes, particularly within the B16-F1 cell line. The inhomogeneity of the cell population might be an explanation for the varying results in our cytometry studies.

Finally, it has to be considered that the adherent B16 cells had to be suspended during cytometry measurements. This might have not only disrupted the pathway of natural $\mathrm{G}_{\mathrm{M} 3}$ expression, but could have also increased the cells' fragility during the staining protocol. Due to the various preparation steps within these procedures, the plasma membrane of the in suspension floating cells could have been damaged and shown defect and inhomogeneous areas, which in turn could have reduced the correct proportion of embedded Gм3 molecules. Following up on this, it was noticeable that the B16-F10 cell line was more sensitive to a longer incubation period with trypsin exhibiting a higher number of dead cells in the medium during the harvesting process compared to the F1 variant.

\subsection{Cell ADHESION PROPERTIES OF THE B16 CELL LINE}

To examine the impact of the glycosphingolipid Gм3 on the adhesion process of the B16-F1 and B16-F10 carcinoma cell line, reflection interference contrast microscopy (RICM) and time-resolved adhesion studies of B16 cells were performed on glass, lyso-LacCer-incorporated solid supported lipid membranes and fibronectin-treated glass substrates. The adhesion of B16 cells on fibronectin is intended to create a natural adhesion environment for the cells. Fibronectin is an adhesive glycoprotein that is a major contributor to the extracellular matrix (ECM). Due to its conformational flexibility, fibronectin acts as a binding partner for a large number of molecules, such as growth factors, cytokines, other ECM components or as a receptor of various signaling cell surface molecules. ${ }^{178,179}$ Because of this characteristic, fibronectin contributes to the maintenance of various vital physiological processes such as embryonic development or wound healing, but also participates in the pathway of abnormal processes such as malignant tumor formation. ${ }^{178,179}$ 
Advantages of the label-free and thus non-invasive RICM are the possibility of optically live-tracking the adhesion points of cells on different surfaces ${ }^{158,159}$ and rules out disturbing effects of fluorescently modified cell components. Due to the influence of different refractive indices and multiple reflections of the objects and substrates however, it is challenging to carry out correct calculations of the cell-substrate distances ${ }^{163,164}$. Therefore, the commonly ${ }^{165,180-182}$ used classification of the color intensities in very close, intermediate and distant areas from the respective substrate is applied to determine the contact areas of the B16 cells. According to IZZARD et al. ${ }^{165}$, very close regions appeared dark gray to black, and have a cell-substrate distance of about 15-30 nm. The dark color results from an interference between the reflected phase-shifted light from the cellular plasma membrane and the reflected light from the glass surface. Cell components, which are about 30-50 $\mathrm{nm}$ away from the surface, have a gray to light gray color and thus characterize the intermediate contact region. Distant adhesion points are nearly white in color and are located about $100 \mathrm{~nm}$ away from the corresponding surface. Here, the reflected light from the cell membrane has a smaller phase shift in contrast to the reflected light from the glass substrate, leading to brighter areas in the image. Components having the same coloration as the background are not in contact with the substrate.

The RICM images shown in Figure 3.5, demonstrate living [A, A'] B16-F1 and [B, B'] B16-F10 cells, adhering to glass or fibronectin-treated substrates. It was noticeable, that the highly metastatic cancer cell line B16-F10, as well as the less invasice cell line B16-F1 showed small and very close contact areas to the surface at their cell borders as indicated by a dark coloration. These are most likely adhesion contacts such as focal adhesions characterized by roundish to partly strip-like structures having a $1.5 \mu \mathrm{m}$ long and $0.25 \mu \mathrm{m}$ wide dimension. ${ }^{183}$ In addition, the images indicated that stress fibers were present at the basal side of the cell, represented as elongated dark regions in the center of the cell. Besides, it seemed like both cell lines adhered closer to the substrate at the cell rim rather than in the center of the cell (cf. Figure 3.5). 

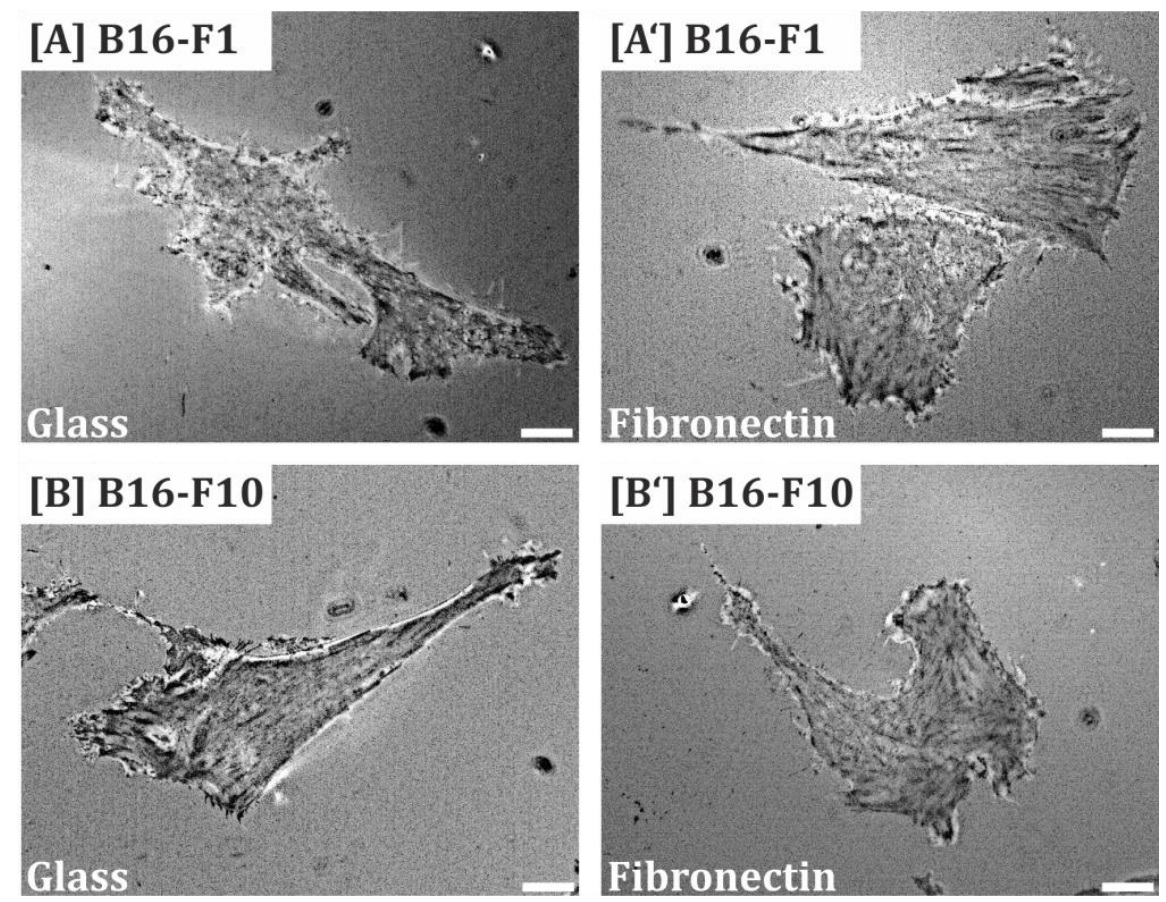

Figure 3.5: Reflection interference contrast microscopy images of separated murine B16 cancer cells grown on different substrates. The B16-F1 variant, grown on [A] glass and [A'] fibronectin-coated surfaces show, as well as the B16-F10 cell line, grown also on [B] glass and [B'] fibronectin-coated surfaces, very close adhesion regions at the cell rims (represented by a dark coloration). Especially in $[\mathrm{A}]$ and $[\mathrm{B}]$ the formation of adhesions points and stress fibers can be demonstrated. Cell seeding density: 50,000 cells per petri dish, grown for $24 \mathrm{~h}$. Scale bars: $10 \mu \mathrm{m}$.

If the adhesive behavior of the cancer cell lines B16-F1 and B16-F10 observed from RICM experiments was compared with the distribution of the ganglioside $\mathrm{G}_{\mathrm{M} 3}$ found in TIRF images (cf. Figure 3.2), a connection between the $\mathrm{G}_{\mathrm{M} 3}$ expression and the close cell adhesion at the cell rims were detected. As the ganglioside was predominantly observed at the cell borders, it can be suggested that $\mathrm{G}_{\mathrm{M} 3}$ tends to be an important player during the adhesion of the B16 cancer cells. This corresponds well to the literature, which states that $\mathrm{G}_{\mathrm{M} 3}$ is enriched at adhesion complexes like focal adhesions ${ }^{26}$, associated with signaling transducer molecules like cSrc, Rho A and focal adhesion kinase ${ }^{25}$ and was identified to mediate the attachment of melanoma to endothelial cells ${ }^{66}$. The assumption that Gm3 plays not only an important role in the adhesion process of B16 cells, but also for the occurrence of serious diseases such as the formation of malignant tumors, was proven in previous studies ${ }^{184}$. Thus, it is known that $\mathrm{G}_{\mathrm{M} 3}$ interacts with caveolin-1, a protein, which is important in cell cycle progression. Its main task is the linkage of integrin to special kinases that promote the cell cycle. In turn, integrins are essential cell adhesion receptors that decisively regulate the interaction between the cell and the surrounding extracellular matrix. With the epidermal growth factor receptor (EGFR) and the plasma membrane $\mathrm{Ca}^{2+}$ 
ATPase (PMCA), further binding partners of the ganglioside were identified. By binding to its receptor, the epidermal growth factor (EGF) stimulates cell growth and differentiation. With regard to cancer research, a variety of studies have already shown that EGFR is upregulated or mutated in certain types of malignant tumors, resulting in uncontrolled growth and increased metastasis. The PMCA is responsible for the removal of calcium ions from the cell. Again, it was shown that a defective function of PMCA can lead to serious diseases.

To gain a clearer picture of the adhesive behavior of the B16-F1 and B16-F10 cells, time-resolved adhesion studies of living B16 cells were performed (cf. Figure 3.6). More specifically, the cells were seeded on $10 \mathrm{~mol} \%$ lyso-LacCer-doted SSLMs and fibronectin-treated glass substrates. By adhesion to fibronectin-coated surfaces, an integrin-mediated adhesion of the cells can be determined, whereas the adhesion to lyso-LacCer-embedded membranes is caused by the interaction between the two glycosphingolipids lyso-LacCer and the cell surface expressed $\mathrm{G}_{\mathrm{M} 3}$. Within $80 \mathrm{~min}$, both cell lines exhibited stronger adhesion to fibronectin-treated surfaces compared to $10 \mathrm{~mol} \%$ lyso-LacCer-containing membranes. This observation is not surprising when considering that the cells are able to provide a large number of fibronectin receptors such as $\alpha_{5} \beta_{1}$-integrin, which in turn lead to a fast and effective anchoring of the cells on fibronectin coated surfaces. ${ }^{178,179,185}$ In contrast, the initial attachment of the B16 cells on the lyso-LacCer-doted SSLMs were mediated by an interaction between lyso-LacCer and $\mathrm{G}_{\mathrm{M}}$. The matrix lipid POPC, which is also embedded in the membrane, is regarded as reaction-inert and therefore does not make any contribution to the cell adhesion. However, the single-bond strength between fibronectin and $\alpha_{5} \beta_{1}$-integrin ( $\left.39 \pm 8 \mathrm{pN}\right)^{186}$ is comparable with the adhesion strength between $\mathrm{G}_{\mathrm{m} 3}$ and lyso-LacCer ( $40 \mathrm{pN}$, cf. Chapter 3.4.1). 


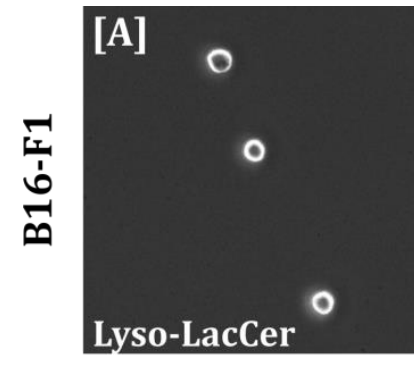

$1 \mathrm{~min}$

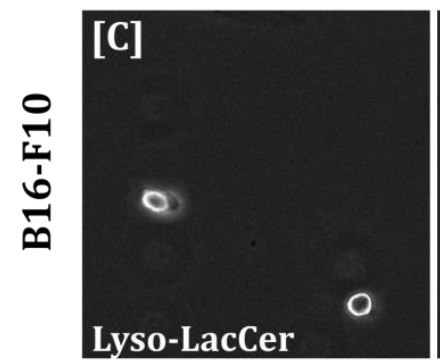

$1 \mathrm{~min}$

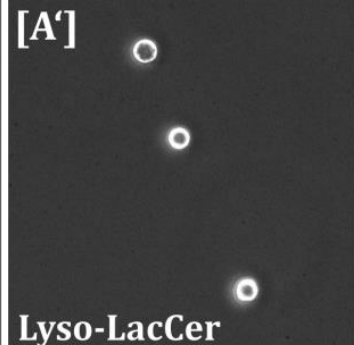

$80 \mathrm{~min}$

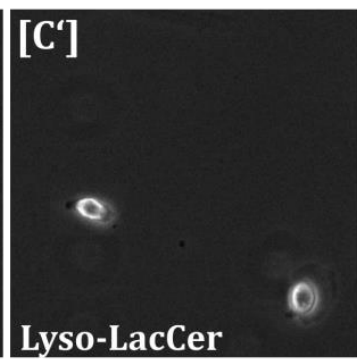

$80 \mathrm{~min}$

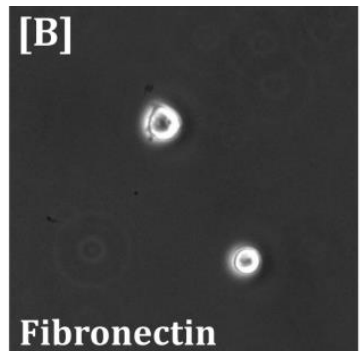

$1 \mathrm{~min}$

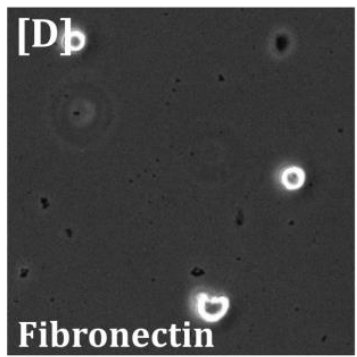

$1 \mathrm{~min}$

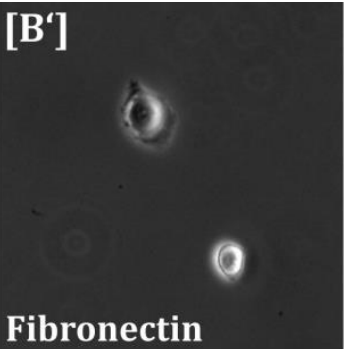

$80 \mathrm{~min}$

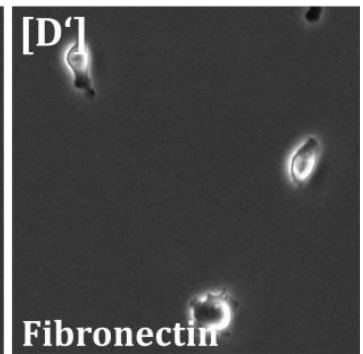

$80 \mathrm{~min}$

Figure 3.6: Phase-contrast images of the adhesive behavior of B16-F1 and B16-F10 cancer cells. Both cell lines were grown on $10 \mathrm{~mol} \%$ lyso-LacCer-containing SSLMs and fibronectin-treated glass supports for $80 \mathrm{~min}$ after cell seeding. The highly metastatic B16-F10 cells showed a more pronounced adhesion on both [D, D'] fibronectin and $\left[\mathrm{C}, \mathrm{C}^{\prime}\right]$ lyso-LacCer-doted SSLMs than the less invasive $\mathrm{F} 1$ variant. For the B16-F1 cells, a spreading process could only be slightly observed on [B, B'] fibronectin surfaces, while on [A, A'] lyso-LacCer-doted SSLMs the cells remained in their spherical initial shape. Cell density: 20,000 cells per petri dish. Scale bar: $100 \mu \mathrm{m}$

Apart from that, it was interesting to see how differently the more metastatic B16F10 cancer cell line behaved on the various substrates compared to the F1 variant. The highly metastatic cell line showed a more effective adhesion on both [C, $\left.\mathrm{C}^{\prime}\right]$ 10 mol\% lyso-LacCer-containing SSLMs and [D, D'] fibronectin. Already 80 min after cell seeding, the B16-F10 cells showed a pronounced spreading on fibronectin, whereas only a few of the B16-F1 cells were able to initiate a spreading process after this time [B, B']. Those differences were even more evident on the 10 mol\% lysoLacCer-doted lipid membranes. Here, the B16-F1 cells did not seem to be able to initiate a spreading process at all [A, A'], whereas, at least partially, the B16-F10 cells were able to form adhesion points on the membrane $\left[\mathrm{C}, \mathrm{C}^{\prime}\right]$. Along with cell studies showing an interaction between $\mathrm{G}_{\mathrm{M} 3}$ and cell adhesive molecules such as integrin receptors $^{62}$, it can be assumed that the highly metastatic B16-F10 cells are more responsive to their environment and might be able to recruit adhesive molecules faster to the basal membrane in contrast to the F1 variant. These observations agree well with our force spectroscopy forces, which propose enhanced adhesion forces between B16-F10 and lyso-LacCer-containing SSLMs (cf. Chapter 3.4.1). 
In literature, the adhesive properties of the B16 melanoma cell line are also a widely discussed topic. In the very beginning when establishing the B16 melanoma cell line as a model system for metastatic cell behavior, FIEDLER ${ }^{5}$ and BOSMANN et al..$^{7}$ observed that the B16-F10 cells produced a greater amount of melanin-containing malignant tumor cells in the lungs of C57BL/6 mice than the less metastatic F1 variant independent from the amount of cells that were injected into the mice. Studies from HILL et al. ${ }^{187}$ supported these results by calculating the effective rate of the ability to generate experimental metastases. For the B16-F1 cell line an effective rate of 1.3 . $10^{-5}$ per cell per generation was found while the F10 variant showed an effective rate of $5.0 \cdot 10^{-5} .187$ Within our studies, we were at least able to confirm a faster and more active adhesion process on 2D surfaces for the highly metastatic F10 variant. To further clarify the contribution of GSLs on the adhesion of the carcinoma B16 cells, single-cell force spectroscopy studies (cf. Chapter 3.4) were performed at early substrate contact times ranging from 0-60 s.

In order to quantify the observations of the enhanced adhesive behavior of the invasive B16-F10 cells and to answer the questions if an interaction between $\mathrm{G}_{\mathrm{M} 3}$ and lyso-LacCer can mediate the initial adhesion of the B16 carcinoma cell lines, singlecell force spectroscopy (SCFS) and colloidal probe microscopy (CPM) studies, based on atomic force microscopy (AFM), were performed (cf. Chapters 3.4.1 and 3.5.1). 


\subsection{DEVELOPMENT OF A MODEL MEMBRANE SYSTEM}

As a comparison to the cell studies, a model membrane system was designed to get a deeper insight into the carbohydrate interactions of $\mathrm{G}_{\mathrm{M} 3}$ and lyso-LacCer. To ensure a natural like environment for $\mathrm{Gm}_{\mathrm{M}}$ and lyso-LacCer, the GSLs were incorporated into solid supported lipid membranes (SSLMs). SSLMs are characterized by a long-term stability 188,189 and are also particularly versatile in the study of membrane processes and the development of biotechnological applications. ${ }^{10,190}$ A common method to create SSLMs is the spreading of vesicles, which adsorb, rupture and fuse together on hydrophilic solid supports. ${ }^{114}$ The charge and topography of the surface, the lipid concentration and the lipid head group as well as the temperature play a crucial role to achieve an optimal spreading process of the vesicles. ${ }^{10,115,191,192}$

In this thesis, GSL-containing SSLMs were prepared by spreading GM3-, LacCer- and lyso-LacCer-doted small unilamellar vesicles (SUVs) on silicon dioxide $\left(\mathrm{SiO}_{2}\right)$ substrates, a process that turned out to be challenging. First, the high phase transition temperature of LacCer $\left(T_{\mathrm{m}} \approx 80^{\circ} \mathrm{C}\right.$, cf. Figure $\left.3.7[\mathrm{~A}]\right)$ caused by high symmetry of the hydrophobic part, made it difficult to create proper SUVs, possibly leading to inefficiently incorporated LacCer into the lipid membrane.

$[\mathrm{A}]$

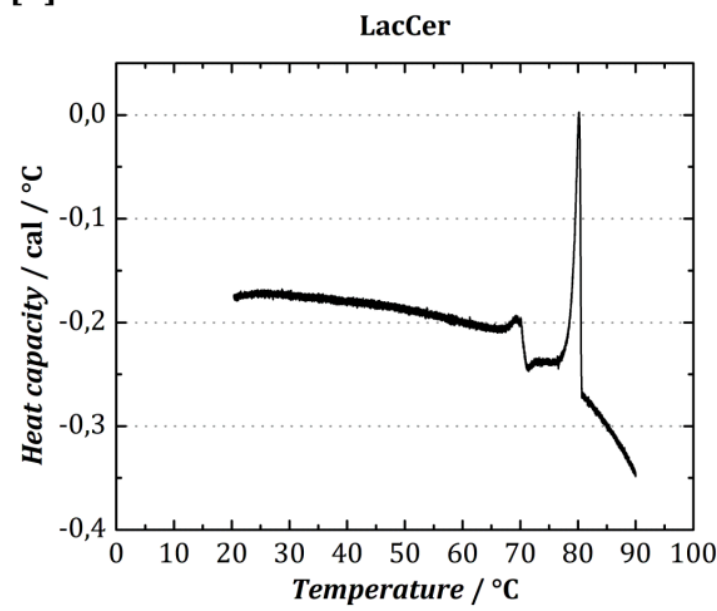

\section{[B]}

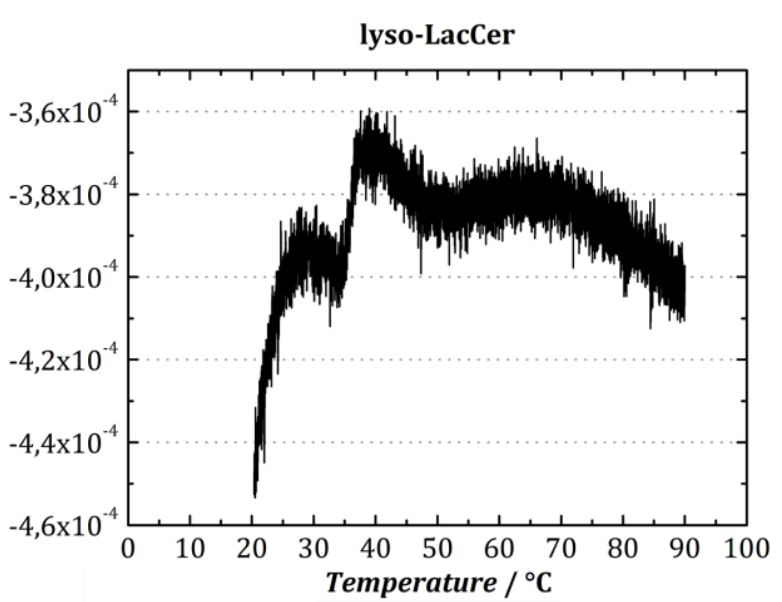

Figure 3.7: Differential scanning calorimetry studies revealing a main phase transition temperature of $T_{\mathrm{m}} \approx 80^{\circ} \mathrm{C}$ for the glycosphingolipids LacCer $[\mathrm{A}]$ and $T_{\mathrm{m}} \approx 40-50{ }^{\circ} \mathrm{C}$ for lyso-LacCer [B]. Both heat scan traces were obtained at scan rate of $15^{\circ} \mathrm{C} / \mathrm{h}$.

To overcome those issues, LacCer was finally replaced by its $N$-deacylated derivative lyso-LacCer. In comparison to LacCer, the hydrophobic part of lyso-LacCer lacks an additional fatty acid chain resulting in a reduced symmetry and lower stabilizing 
forces of the hydrophobic part of the lipid. As a consequence, the main phase transition temperature decreased to $T_{\mathrm{m}} \approx 40-50{ }^{\circ} \mathrm{C}$ (cf. Figure 3.7[B]). However, pure lyso-LacCer exhibited a much lower solubility during the formation of multilamellar vesicles (MLVs) for DSC studies, so that recording of heat scans was considerably more difficult, since strong noise was present in all experiments. To ensure the embedding of lyso-LacCer in the membrane, all necessary preparation steps were performed at an ambient temperature of $70^{\circ} \mathrm{C}$.

The ganglioside $\mathrm{G}_{\mathrm{M} 3}$ shares an almost identical symmetry and composition with LacCer, but differs in the structure of its polar head group. With an additional charged large sialic acid residue, $\mathrm{G}_{\mathrm{M} 3}$ reveals a larger head group structure resulting in decreased phase transitions at around $T_{\mathrm{M}}=35^{\circ} \mathrm{C}^{47,95-97}$ Consequently, the setting of an ambient temperature of $40{ }^{\circ} \mathrm{C}$ was sufficient to prepare proper Gм3-doted lipid membranes. For the matrix lipid POPC, main phase transition temperatures of $T_{\mathrm{M}}=-2{ }^{\circ} \mathrm{C}^{87-89}$ were found, thus the spreading procedure was carried out at room temperature. Since subsequent CPM experiments were carried out at room temperature or at $37^{\circ} \mathrm{C}$ for SCFS studies, it was essential to ensure that the membrane was not subjected to severe temperature fluctuations after preparation. This could have disrupted the characteristic phase transition of the lipids and thus distort their specific lateral distribution. For this reason, the formed lipid membranes were slowly cooled down to room temperature overnight.

Further, it was pivotal to overcome the electrostatic repulsion between the charged lipid head groups and the charged $\mathrm{SiO}_{2}$ substrate surface. To reduce the repulsive interactions during the spreading process of GSL-doted SUVs on $\mathrm{SiO}_{2}$, positively charged $\mathrm{Ca}^{2+}$ ions were added during membrane formation. Spreading vesicles in presence of divalent cations is a conventional preparation method and facilitates the formation of stable lipid membranes. ${ }^{10,117,193,194}$ The attempt to diminish the negative charge of the surface by lowering the surrounding $\mathrm{pH}$ value ${ }^{195}$ proved ineffective. Although $\mathrm{Ca}^{2+}$ ions were essential for the spreading process, they may also cause premature aggregation of the incorporated GSLs, since $\mathrm{Ca}^{2+}$ ions have been shown to enhance carbohydrate-carbohydrate interactions between GSLs in neighboring cells or model systems. ${ }^{53,196,197}$ Therefore, $\mathrm{Ca}^{2+}$ ions were entirely omitted during SUV formation and reduced to a low amount during the spreading process. To verify the 
impact of $\mathrm{Ca}^{2+}$ ions on the formation of GSL-doted SSLMs and on the interaction between the glycosphingolipids $\mathrm{G}_{\mathrm{M} 3}$ and lyso-LacCer, the freshly prepared membranes were incubated with $\mathrm{Ca}^{2+}$ and EDTA-containing buffer (cf. Table 2.2, Chapter 2.2.3). EDTA is able to form very stable 1:1 chelate complexes with divalent cations ${ }^{198}$ and is applied to remove present $\mathrm{Ca}^{2+}$ ions when required. A possible effect of the buffer components as well as on the quality of the lipid membranes was verified by fluorescence and atomic force microscopy. For visualization, the photostable and highly absorbent ${ }^{98,100}$ fluorescently labeled lipids $\beta$-Bodipy ${ }^{\mathrm{TM}}$ and Texas Red $^{\mathrm{TM}}$ DHPE were additionally embedded into the lyso-LacCer- or $\mathrm{G}_{\mathrm{M} 3}$-containing membranes.

Fluorescence recovery after photobleaching (FRAP) experiments were performed to determine the quality of the lipid membrane formed in a calcium-containing and noncontaining environment. In Figure 3.8, FRAP time series of [A] a 10 mol\% lyso-LacCer, [B] a 2 mol\% lyso-LacCer- and [C] a 10 mol\% GM3-incorporated SSLM on silicon substrates in the presence of calcium ions are shown. All three membranes revealed a rather homogeneous fluorescence throughout the entire area. To perform FRAP, the homogenous $\beta$-Bodipy ${ }^{\mathrm{TM}}$ or Texas Red ${ }^{\mathrm{TM}}$ DHPE dyes were irreversibly photooxidized in a defined region of interest (ROI, yellow) by a short laser pulse at time $t=0 \mathrm{~s}$. In the case of a fluid lipid membrane, intact neighboring fluorescently labeled lipids are able to diffuse into the bleached region leading to a recovery of the fluorescence intensity. The recovery of the fluorescence intensity in the bleached area was observable in all three membranes and a representative intensity time trace is shown in Figure 3.8 [D] for a 10 mol\% lyso-LacCer-doted SSLM indicating the formation of fluid GSL-embedded membranes. However, the presence of impurities, which are recognizable by spots with increased fluorescence intensity, could not be avoided as exemplary shown in Figure $3.8[\mathrm{~A}]$ or [B]. Since those objects showed no fluorescence recovery after being bleached, it can be concluded that loosely adsorbed vesicles populate the surface of the underlying lipid bilayer. Similar results were observed with EDTA-incubated GSL-containing membranes (cf. Figure 6.1, Chapter 6). 

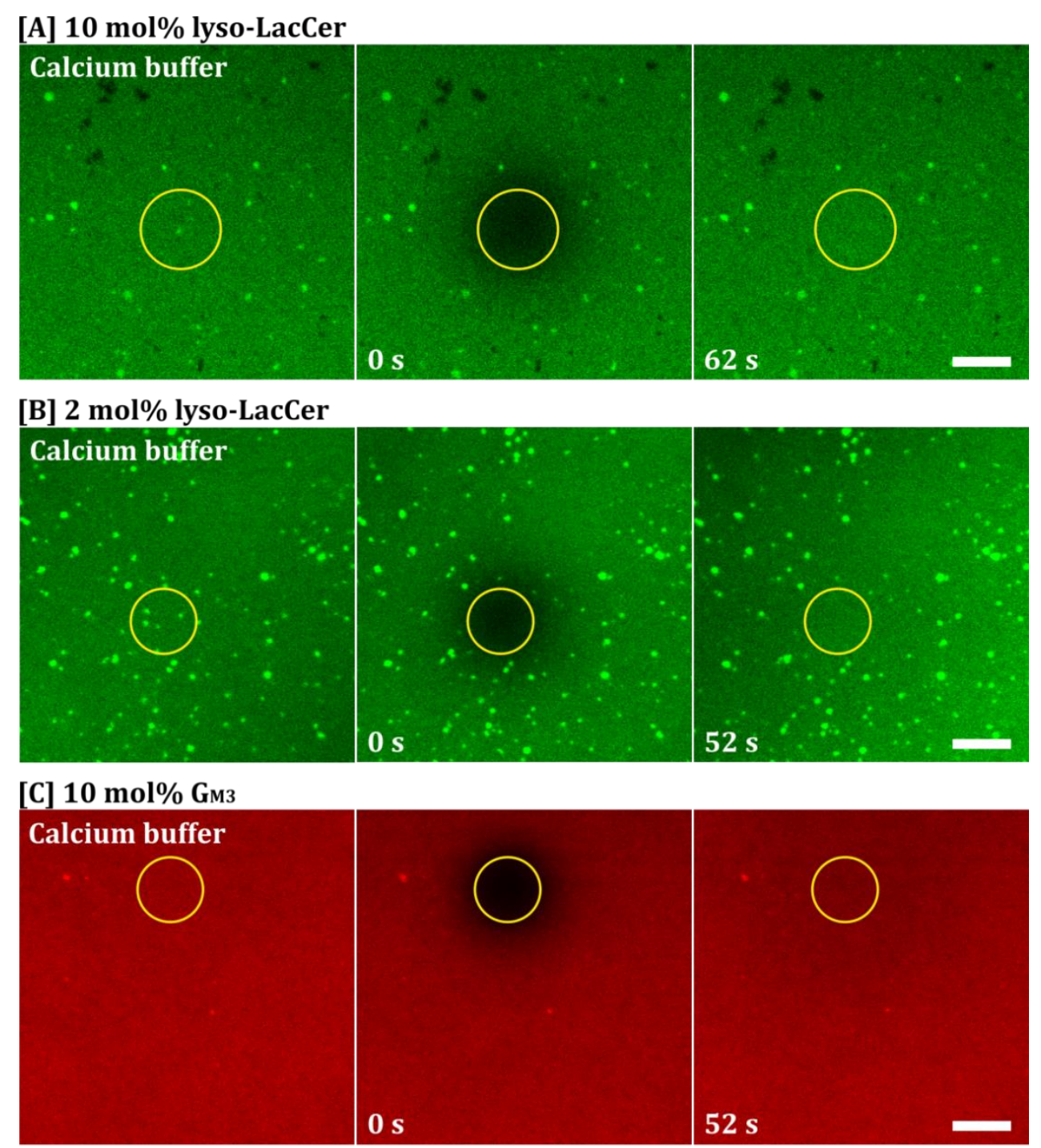

\section{[D] FRAP curve}

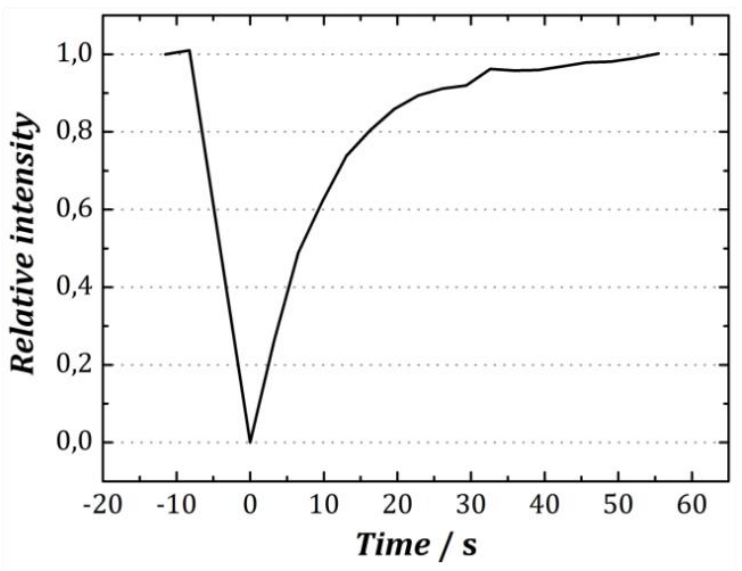

Figure 3.8: Images showing time series of a fluorescence recovery after photobleaching (FRAP) experiment on solid supported lipid membranes consisting of [A] POPC/lyso-LacCer/ $\beta$-Bodipy ${ }^{\mathrm{TM}}(89: 10: 1 \mathrm{~mol} \%)$, [B] POPC/lysoLacCer/ $\beta$-Bodipy ${ }^{\mathrm{TM}}$ (97:2:1 mol\%) and [C] POPC/Gм3/Texas RedTM DHPE (89:10:1 mol\%) on silicon dioxide substrates. The quality of the membranes was analyzed in the presence of a calcium-containing buffer. The fluorescent molecules in a desired region of interest (ROI, yellow) were irreversibly bleached at time $t=0$. Due to the diffusion of surrounding intact fluorophores, the fluorescence intensity was recovered within the ROI. [D] Exemplary FRAP curve of the membrane shown in [A]. Here, the observed fluorescence intensities from the specific ROI were normalized to the fluorescence intensity at the beginning and plotted against the time. Scale bars: $5 \mu \mathrm{m}$. 
In addition, the FRAP techniques allows to estimate the lateral mobility of fluorescent molecules by determining the specific diffusion coefficients $D$ and the mobile fractions $F_{m}$ (cf. Chapter 2.8.3.1). Within this project, the above mentioned parameters were only calculated once for each membrane $(N=1)$ according to AXELROD et al. ${ }^{152}$ Thus, only first indications of the mobility of the lipids can be given. The calculated diffusion coefficients $D$ and mobile fractions $F_{m}$ of the various membranes in presence (cf. Figure 3.8) and absence (cf. Figure 6.1, Chapter 6) of calcium ions are shown in Table 3.1. The high mobile fraction indicates very fluid GSLcontaining membranes, whereas the diffusion of the fluorophores seems to be rather slow compared to reported diffusions coefficients of phosphatidylcholine lipid membranes $\left(D \approx 0.5-5 \mu \mathrm{m}^{2} \cdot \mathrm{s}^{-1}\right) .{ }^{199,200}$

Table 3.1: Overview of diffusion coefficients $D$ and mobile fractions $F_{\mathrm{m}}$ of various membranes in calcium- or EDTAcontaining buffer. Both parameters were determined at room temperature. The errors were calculated by Gaussian error propagation of the uncertainties of the fit parameters. $N=1$ for each tested condition.

\begin{tabular}{cccc}
\hline & & $\boldsymbol{D} / \boldsymbol{\mu m}^{2} \cdot \mathbf{s}^{-1}$ & $\boldsymbol{F}_{\mathbf{m}}$ \\
\hline \hline \multirow{4}{*}{ Calcium } & 2 mol\% lyso-LacCer & $0.382 \pm 0.028$ & $0.941 \pm 0.015$ \\
& $10 \mathrm{~mol} \% \mathrm{GM} 3$ & $0.230 \pm 0.014$ & $0.879 \pm 0.017$ \\
& 10 mol\% lyso-LacCer & $0.345 \pm 0.008$ & $0.984 \pm 0.005$ \\
EDTA & 2 mol\% lyso-LacCer & $0.309 \pm 0.015$ & $0.962 \pm 0.012$ \\
& 10 mol\% GM3 & $0.248 \pm 0.011$ & $0.852 \pm 0.011$ \\
\hline
\end{tabular}

Previous literature $91,201,202$ and DSC studies performed within this thesis reveal that even small GSL have high phase transition temperatures compared to corresponding phosphatidylcholine or sphingomyelin moieties. As described in Chapter 3.1, GSLs exist in a solid-like $\left(S_{0}\right)$ gel phase under physiological conditions and form GSLenriched microdomains (GEM) due to side-by-side interactions. To gain more information about potential GEM formation in GSL-doted solid supported lipid membranes, topography studies of the membrane surfaces were performed using atomic force microscopy. Figure 3.9 shows topographical maps of GSL-containing membranes as well as a pure POPC membrane in the presence (calcium buffer) and absence (EDTA buffer) of calcium ions. 
[A] POPC

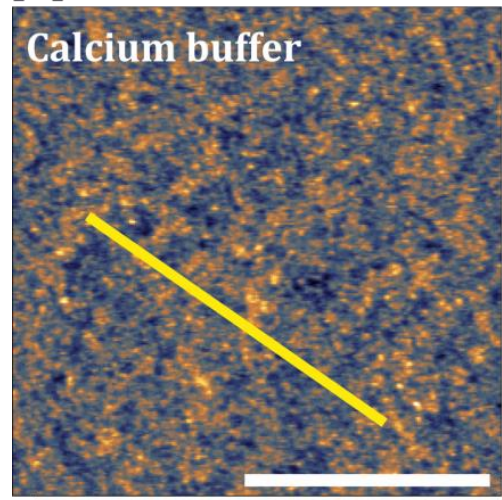

[B] $10 \mathrm{~mol} \%$ lyso-LacCer [C] $10 \mathrm{~mol} \% \mathrm{Gm3}$
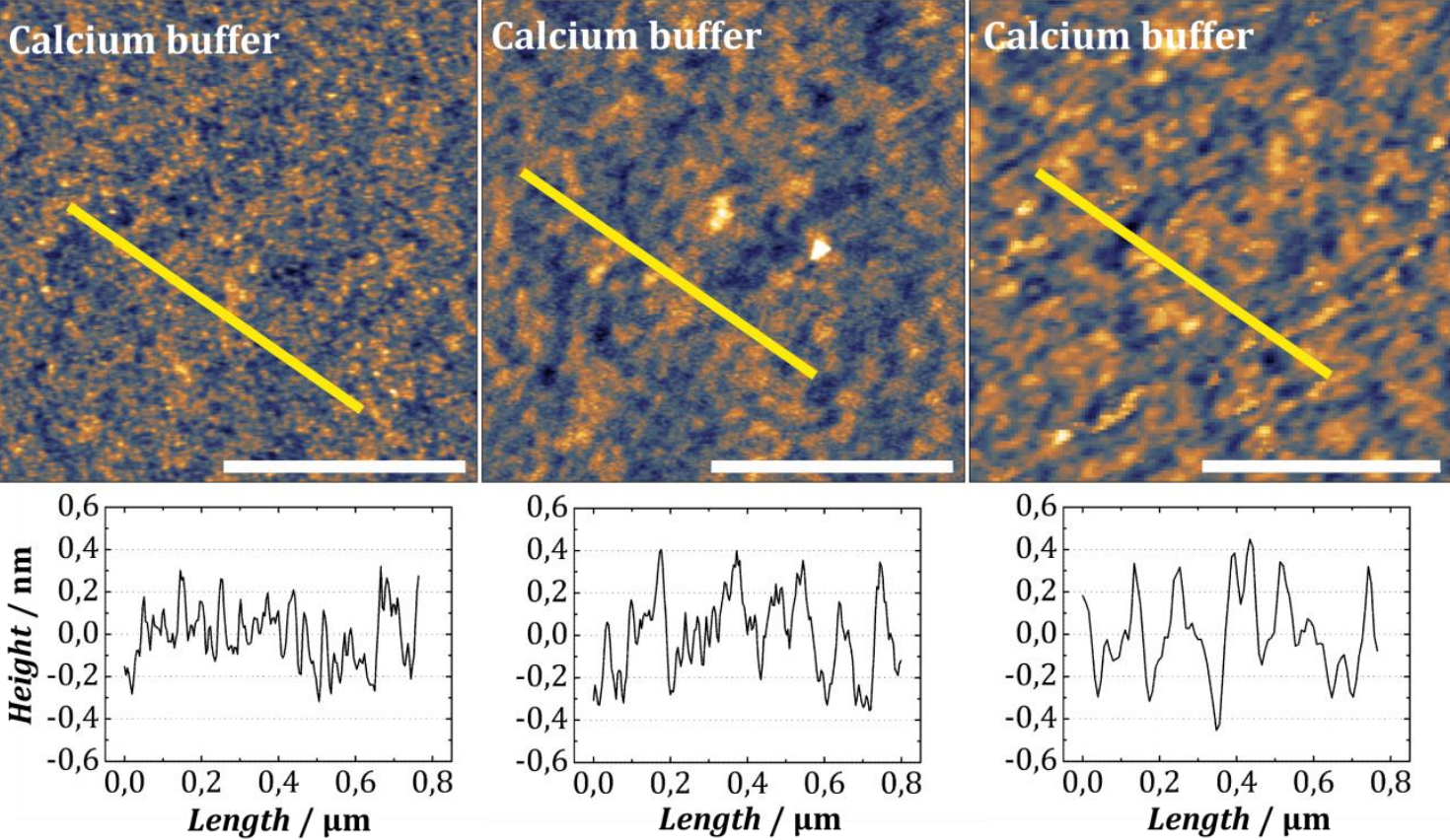

[D] 2 mol\% Lyso-LacCer

[E] $10 \mathrm{~mol} \%$ lyso-LacCer
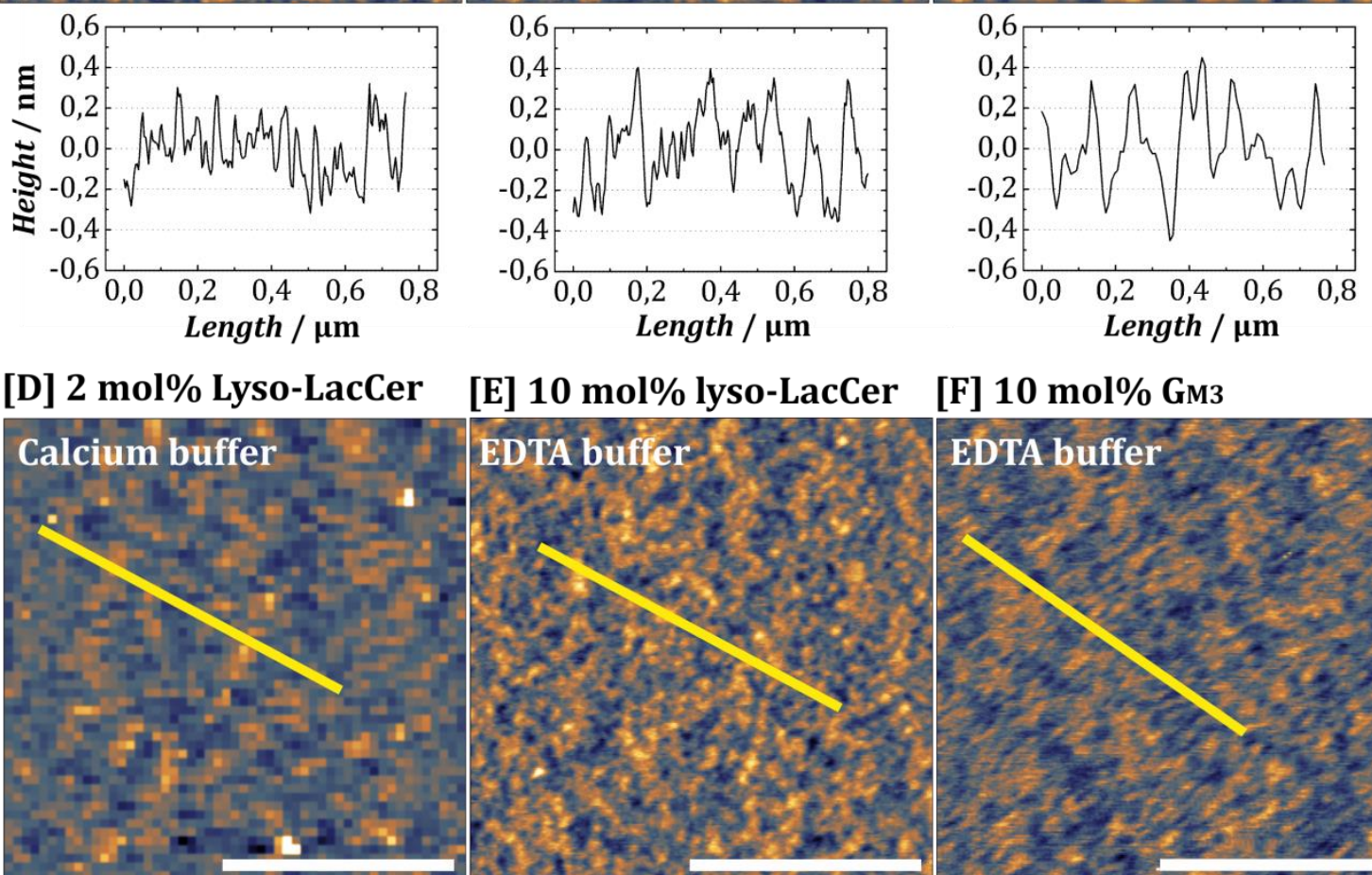

[F] $10 \mathrm{~mol} \%$ GM3
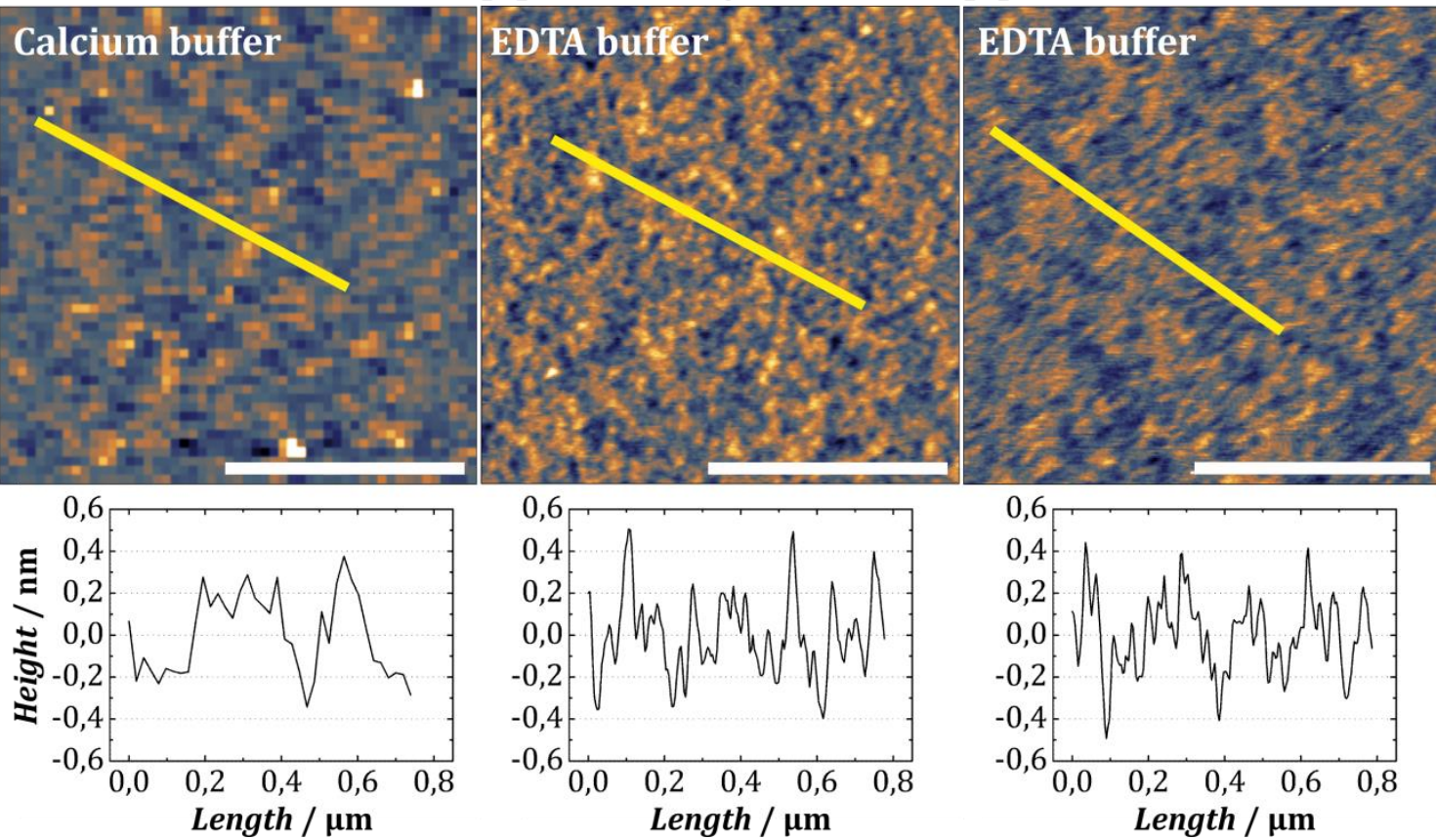

Figure 3.9: Exemplary topographical maps (AFM, tapping mode) of lipid membranes consisting of [A] POPC,

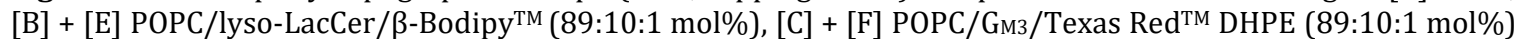
and [D] POPC/lyso-LacCer/ $\beta$-Bodipy ${ }^{\mathrm{TM}}(97: 2: 1 \mathrm{~mol} \%)$ on silicon dioxide substrates. The topography of the membranes was analyzed in presence (calcium buffer) and absence (EDTA buffer) of calcium ions and reveals the formation of higher structures (orange to gold). The height profiles of those structures correspond to the marked yellow lines. Scale bar: $500 \mathrm{~nm}$.

Although the fluorescence images (cf. Figure 3.8) indicated a relatively planar membrane surface, slight topographic differences were found in the AFM images caused by the selection of the GSL moiety and the GSL concentration. Compared to the rather homogenous and relatively flat surface topography ( $\sim 0.2 \mathrm{~nm}$ high structures) 
of the pure POPC membrane (cf. Figure $3.9[\mathrm{~A}]$ ), a greater amount of increased structural elements ( 0.2-0.4 nm high structures) was detected on the surface of the 10 mol\% lyso-LacCer-containing membrane (cf. Figure 3.9 [B]). In addition, those objects seemed to accumulate in certain areas, indicating the formation of GEMs. Objects with similar height values can be observed within the $10 \mathrm{~mol} \%$ Gмз-doted membrane (cf. Figure $3.9[\mathrm{C}]$ ) revealing the formation of even larger domain sizes. By reducing the lyso-LacCer content to 2 mol\% (cf. Figure 3.9 [D]), these structures tend to lose their accumulation in specific domains and appear to be more homogeneously distributed throughout the membrane. However, the height of the structures ( $\sim 0.2-0.4 \mathrm{~nm})$ seems to be more similar to the height values of the objects in GSLdoted membranes than in POPC membranes. In the absence of calcium ions the observed structures tend to accumulate less within the $10 \mathrm{~mol} \%$ lyso-LacCer- and $\mathrm{G}_{\mathrm{M} 3}$-doted membranes (cf. Figure $3.9[\mathrm{E}]+[\mathrm{F}]$ ), but still revealing height structures of $\sim 0.2-0.5 \mathrm{~nm}$. Thus, it can be assumed that the obtained higher structures (goldcolored) correspond to GSLs embedded into the leaflet of the solid supported membrane, which offer larger and more complex head group structures compared to POPC. Similar results were obtained in studies examining the binding of pentameric cholera toxin B subunits (CTBs) to the corresponding ganglioside GM1 membrane ligand. ${ }^{203}$ By means of AFM experiments, SHI et al. ${ }^{203}$ found $\sim 1.0-2.0 \mathrm{~nm}$ high and 15-60 nm large $G_{M 1}$ clusters in supported POPC bilayers in the absence of CTB, depending clearly on the $\mathrm{G}_{\mathrm{M} 1}$ density (from 0-10 mol\%) within the membrane. The height of the obtained structures were in good agreement with the size of the pentasaccharide head group of $\mathrm{G}_{\mathrm{M} 1}$, determined by X-ray diffraction ${ }^{204,205}$. Compared to the smaller di- and trisaccharide head groups of lyso-LacCer and $\mathrm{G}_{\mathrm{M} 3}$ and the fact that already small changes within acyl chain and head group structures of GSLs show a significant impact on GSL behavior in microdomains ${ }^{38,206,207}$, it seems reasonable that smaller structure and domain sizes of lyso-LacCer and $\mathrm{G}_{\mathrm{M} 3}$ were detected within AFM studies of this thesis.

Although the distribution and organization of lyso-LacCer and $\mathrm{G}_{\mathrm{M} 3}$ and the possible accumulation of those GSLs in GEMs must be investigated by further studies, it can be concluded that the model membrane system presented here is quite capable of producing intact and reproducible GSL-containing membranes. 


\subsection{SINGLE-CELL FORCE SPECTROSCOPY OF B16 CELLS}

\subsubsection{QUANTIFICATION AND VALIDATION OF THE CARBOHYDRATE- CARBOHYDRATE INTERACTION}

In Chapter 3.2, the adhesion capabilities of the GM3-expressing melanoma cancer cell lines B16-F1 and B16-F10 were discussed. Particular emphasis was placed on the contribution of the ganglioside $\mathrm{G}_{\mathrm{M} 3}$, which interacts with the GSL lyso-LacCer in a $\mathrm{Ca}^{2+}$ containing environment. Based on the fundamentals of atomic force microscopy (AFM), single-cell force spectroscopy (SCFS) was used to investigate the adhesion of individual, living cells at the molecular level and especially to determine the forces between interacting molecules. For this purpose, single B16-F1 or B16-F10 cells were attached to a cantilever using positively charged $\alpha$-amino acid poly-D-lysine (cf. Chapter 2.6.1.1) and brought into contact with lyso-LacCer-containing or -lacking SSLMs on glass. Then, the detected forces upon retraction of the cantilever were recorded to gain information about the maximum adhesion forces between the cell and a lyso-LacCer-doted lipid membrane. Figure 3.10 shows an example of an attached single B16-F10 cell on the cantilever tip pressed onto a $1 \mathrm{~mol} \%$ lyso-LacCercontaining lipid membrane for $45 \mathrm{~s}$.

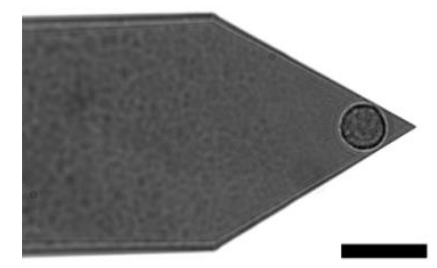

Figure 3.10: Image of a single B16-F10 cell brought in contact with a 1 mol\% lyso-LacCer-containing lipid membrane for $45 \mathrm{~s}$. The cell was attached to a cantilever tip, which was coated with the $\alpha$-amino acid poly-D-lysine. Scale bar: $40 \mu \mathrm{m}$.

For the investigation of the carbohydrate-carbohydrate interaction between the Gм3expressing cell and lyso-LacCer, the respective cell was pressed on $1 \mathrm{~mol} \%$ and $10 \mathrm{~mol} \%$ lyso-LacCer-doted membranes (for parameters cf. Table 2.6, Chapter 2.6.1.1). Three control measurements were performed to verify the specific interaction between Gм3 and lyso-LacCer. On the one hand, forces between cells and pure POPC membranes were measured, whereas on the other hand, cells were treated either with a monoclonal IgM antibody against $\mathrm{G}_{\mathrm{M} 3}$ or with the enzyme sialidase (cf. 
Chapter 2.1.2.2) to remove sialic acid residues in the Gм3 molecule and pressed onto a $10 \mathrm{~mol} \%$ lyso-LacCer-containing SSLM. Before force measurements were performed, the cells were examined for its vitality by optical microscopy. In addition, the respective cell contact areas (cf. Table 3.2) were determined to observe size differences between B16-F1 and B16-F10 cancer cells, which might result from treatment with the antibody and the enzyme. Especially the determination of the respective contact areas is important for the correct interpretation of the measured forces, as strongly enlarged contact regions could lead to more potential binding partners, which in turn could lead to increased forces between the cell and the substrate. In Table 3.2 the respective contact areas are listed that resulted from direct contact with the substrate.

Table 3.2: Overview of contact areas of untreated or treated B16-F1 and B16-F10 cells, pressed on 1 mol\% lysoLacCer-doped or pure POPC membranes for at least 30-45 s.

\begin{tabular}{|c|c|c|c|c|}
\hline & Lyso-LacCer & & POPC & \\
\hline & Contact area / $\mu \mathrm{m}^{2}$ & $N$ & Contact area / $\mathrm{\mu m}^{2}$ & $N$ \\
\hline $\begin{array}{c}\text { B16-F1 } \\
\text { untreated }\end{array}$ & $192 \pm 16$ & 3 & $204 \pm 58$ & 3 \\
\hline $\begin{array}{l}\text { B16-F1 } \\
\text { Anti-G } \mathrm{G}_{\mathrm{M} 3}\end{array}$ & $205 \pm 23$ & 2 & & \\
\hline $\begin{array}{l}\text { B16-F1 } \\
\text { Sialidase }\end{array}$ & $181 \pm 18$ & 4 & & \\
\hline $\begin{array}{l}\text { B16-F10 } \\
\text { untreated }\end{array}$ & $184 \pm 38$ & 4 & $150 \pm 15$ & 3 \\
\hline $\begin{array}{c}\text { B16-F10 } \\
\text { Anti-G } G_{M 3}\end{array}$ & $229 \pm 21$ & 2 & & \\
\hline $\begin{array}{c}\text { B16-F10 } \\
\text { Sialidase }\end{array}$ & $203 \pm 11$ & 3 & & \\
\hline
\end{tabular}

Note that untreated B16 cells were brought in contact with 1 mol\% lyso-LacCerdoped or pure POPC membranes, while B16 cells treated with the anti-Gм3 antibody or the enzyme sialidase were pressed onto $10 \mathrm{~mol} \%$ lyso-LacCer-incorporated membranes. It was found that both the treatment of the cells, as well as the different metastasis potentials of the B16 cell line in general do not have a large influence on the contact area. Only with POPC, the contact areas showed a slight difference between the B16-F1 and B16-F10 cell line. We conclude that the contact area is mainly a result of normal force paired with the cortical tension of the cell. Also, for this condition the error range is overlapping. So, no major contact area bias were detected. 
[A]

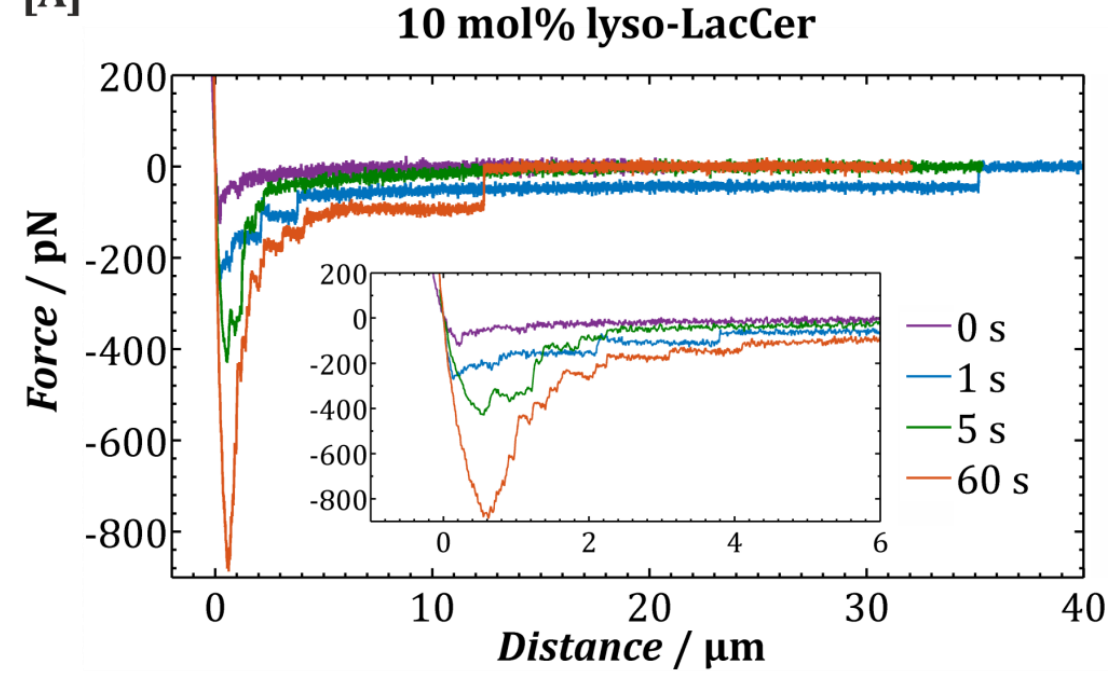

[B]

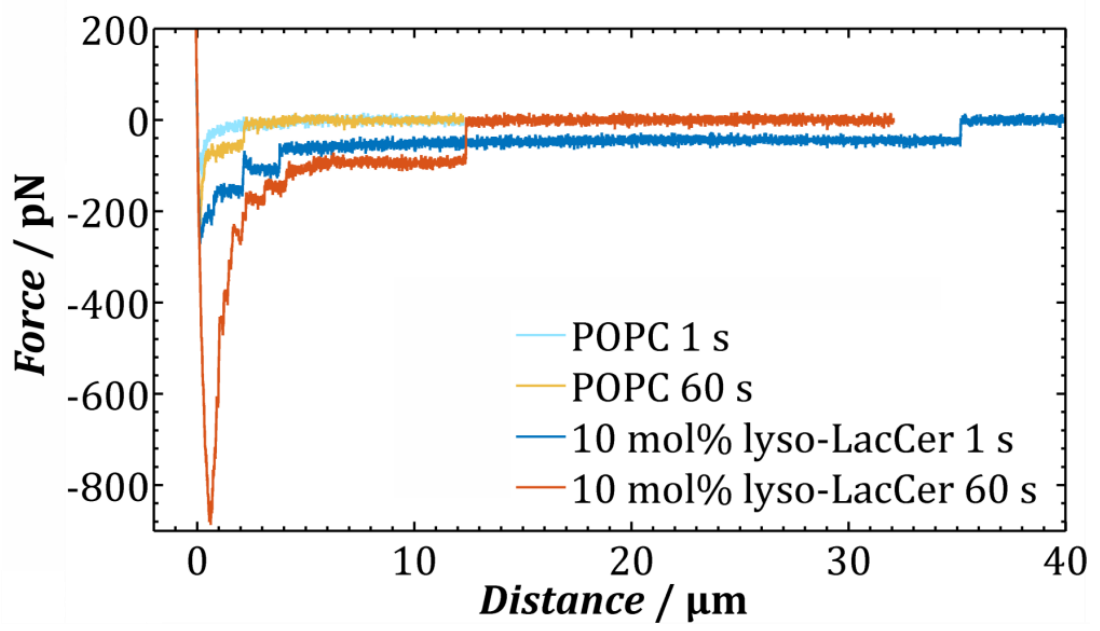

Figure 3.11: Exemplary force-distance curves recorded with single-cell force spectroscopy demonstrating the maximum adhesion forces between the B16-F10 cell line and a $10 \mathrm{~mol} \%$ lyso-LacCer-containing or pure POPC lipid membrane. In $[\mathrm{A}]$ typical force-distance curves are shown presenting the binding strength of the cell and lyso-LacCer after various contact times of $0 \mathrm{~s}$ (violet), $1 \mathrm{~s}$ (blue), $5 \mathrm{~s}$ (green) and $60 \mathrm{~s}$ (orange). In [B], the maximum adhesion forces of the cell-lyso-LacCer interaction detected after $1 \mathrm{~s}$ and $60 \mathrm{~s}$ are compared to the adhesion affinity between the cell and a pure POPC lipid membrane after the same contact times (shown in light blue or light orange). The adhesion forces increase with longer contact times and show higher force values if the cell is able to interact with lyso-LacCer compared to POPC.

To assess the influence of the carbohydrate-carbohydrate-mediated interactions on the adhesion of the B16-F1 and B16-F10 cancer cells, the maximum adhesion forces between the cell and the respective substrate were analyzed by SCFS. Different cellsubstrate contact times between 0-60 s provided information about possible cellinternal initial adhesion processes caused by the $\mathrm{G}_{\mathrm{M} 3}$-lyso-LacCer interaction. In Figure 3.11 exemplary forces-distance curves recorded at contact times of $0 \mathrm{~s}$ (violet), $1 \mathrm{~s}$ (blue) $5 \mathrm{~s}$ (green) and $60 \mathrm{~s}$ (orange) are shown, demonstrating the binding strength between the metastatic B16-F10 cells and [A] a $10 \mathrm{~mol} \%$ lyso-LacCercontaining or $[\mathrm{B}]$ a pure POPC lipid membrane. They indicate a correlation between 
the contact times and maximum adhesion forces and contain information about possible unbinding processes between the cell and lyso-LacCer. Especially in the force-distance curves for contact times greater than $1 \mathrm{~s}$, it can be seen that during the separation of the cantilever tip from the surface two types of unbinding events (cf. Chapter 2.6) occurred. Unbinding effects, which exhibit a non-linear force increase before an instantaneous force decrease are commonly described as jumplike rupture steps $^{135,136}$ and correspond to receptors, which are mostly inserted into the cellular membrane and are also anchored in the cytoskeleton via other proteins. A large number of these jumplike rupture steps $(\sim 40-60 \mathrm{pN}$, cf. Figure 6.3, Chapter 6$)$ were identified in the force-distance curves representing long contact times (cf. Figure 3.11 [A]). The second type of unbinding events is the formation of tetherlike steps, which occur when membrane nanotubes are pulled out of the cellular surface. Tetherlike steps are characterized by long plateaus of constant forces before also instantaneous force decreases due to the constant tension of the cellular membrane ${ }^{135,136}$ were detected. These tetherlike steps were more frequently observed at longer contact times (cf. Figure 3.11 [A]). Comparing the maximum adhesion forces obtained during the interaction between the B16-F10 cells and pure POPC membranes (cf. Figure 3.11 [B], light blue and light orange curve), the force-distance curves revealed stronger adhesion of the cells to lyso-LacCer-containing membranes than pure POPC membranes. For POPC, only a slight increase of maximum adhesion forces were observed with longer contact times.

Figure 3.12 represents an overview of the mean maximum adhesion forces of the B16F1 and B16-F10 cell line obtained with SCFS at contact times ranging from 0-60 s. The distribution of all measured maximum adhesion forces of both cell lines for each contact time is shown in Figure 3.13 and Figure 3.14 as well as in Figure 6.4 and Figure 6.5. It was found that the mean maximum adhesion forces, which occurred at contact times smaller than $1 \mathrm{~s}$, differed markedly from the forces that were measured at contact times between 5-60 s. The plot clearly shows that a strong adhesion of the B16-F1 and B16-F10 cells only occurred when lyso-LacCer had been embedded into the substrate. This is shown by the fact that B16-F1 (cf. Figure 3.12 [A]) and B16-F10 (cf. Figure $3.12[\mathrm{~B}]$ ) cells in contact with pure POPC membranes (blue line) only revealed mean maximum adhesion forces of about $500 \mathrm{pN}$ even at contact times of up 
to $60 \mathrm{~s}$. Similar results were observed when the cells were treated with the anti-Gм3 antibody (red line), or in the case of B16-F10, with the enzyme sialidase (green line). In contrast, non-control force experiments with $1 \mathrm{~mol} \%$ or $10 \mathrm{~mol} \%$ lyso-Lacer-doted membranes showed significantly increased mean maximum adhesion forces at longer contact times. Within studies of the B16-F1 cells (cf. Figure 3.12 [A]), these forces settled at 700 pN (1 mol\% lyso-LacCer, violet line) or 750 pN (10 mol\% lyso-LacCer, black line). On the other hand, the B16-F10 cells (cf. Figure 3.12 [B]) showed markedly increased adhesion forces up to $1000 \mathrm{pN}$ (1 mol\% lyso-LacCer, violet line) or even $1800 \mathrm{pN}$ (10 mol\% lyso-LacCer, black line). These results indicated that the carbohydrate-carbohydrate interactions were able to mediate cell attachment for the B16 cancer cells and that B16-F10 cells showed larger adhesion strengths than the F1 variant.

[A]

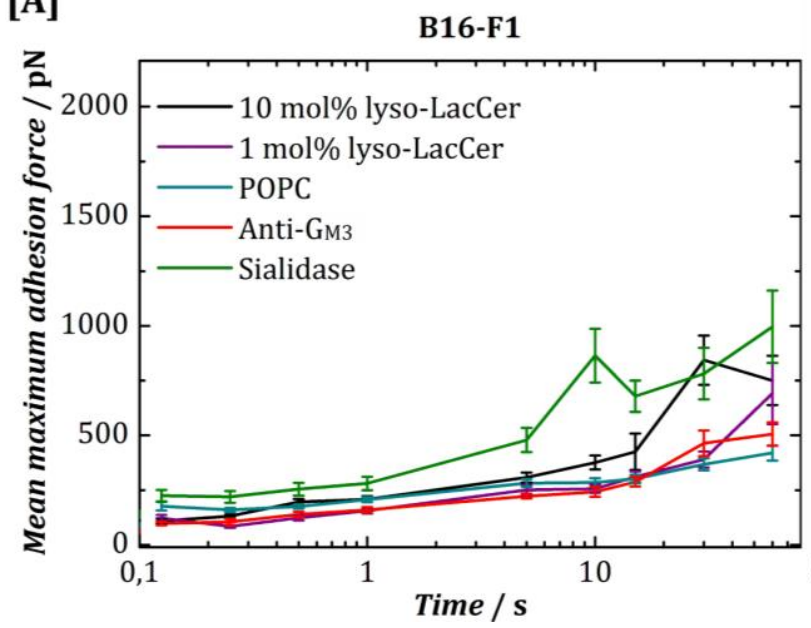

[B]

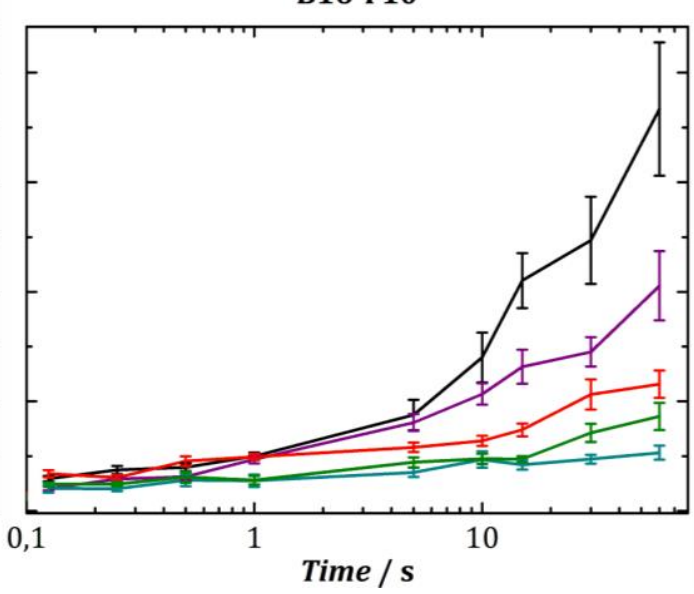

Figure 3.12: Mean maximum adhesion forces between the B16-F1 and B16-F10 cell line and the respective substrate in dependency of a given contact time ranging from 0-60 s (logarithmic scale). Black and violet lines represent adhesion forces, which resulted from the interaction between the respective cells and a solid supported lipid membrane containing $10 \mathrm{~mol} \%$ or $1 \mathrm{~mol} \%$ lyso-LacCer. Mean maximum adhesion forces illustrated in blue demonstrate the contact between the cells and a pure POPC lipid membrane, whereas red and green lines show mean maximum adhesion forces of anti-Gм3 antibody or sialidase treated B16-F1 or B16-F10 cells, which were pressed onto 10 mol\% lyso-LacCer-embedded lipid membranes. Measurements include about 5 cells and 15-25 force curves per category.

In the following section, the distribution of the maximum adhesion forces of the highly metastatic B16-F10 cancer cell line will be discussed and compared with the obtained results within the less invasive B16-F1 variant. To demonstrate the distribution of individual maximum adhesion force values, the data was plotted in a box plot diagram (cf. Figure 3.13, Figure 3.14, Figure 6.4 and Figure 6.5), including the mean (represented as squares in the respective color) and median (represented as solid line in the respective color) of the maximum adhesion forces. 


\section{[A]}

$10 \mathrm{~mol} \%$ lyso-LacCer

$\left[A^{\prime}\right]$

$10 \mathrm{~mol} \%$ lyso-LacCer
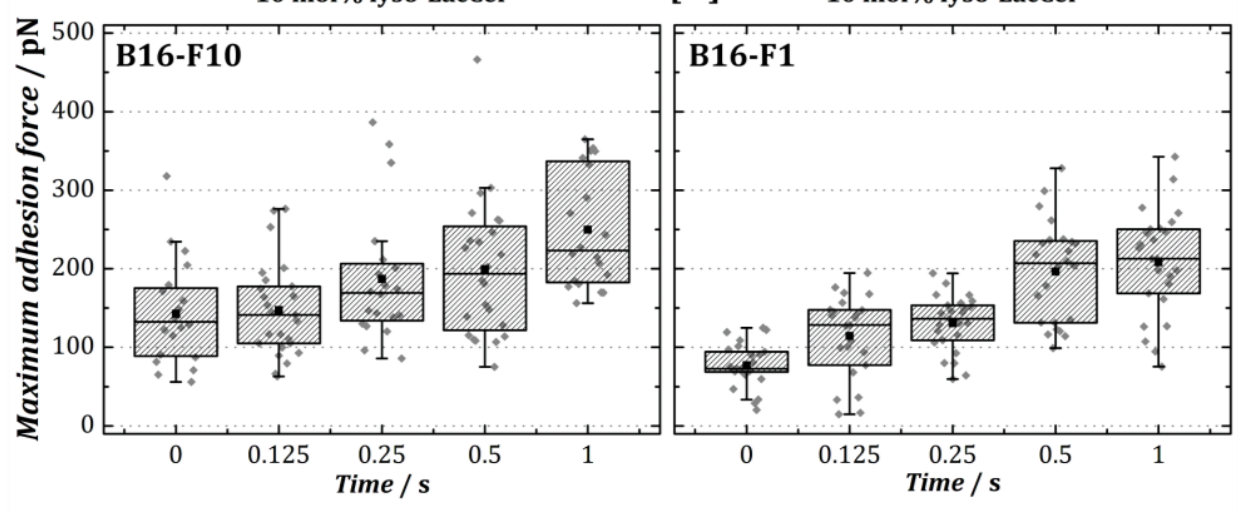

[B]

[C]
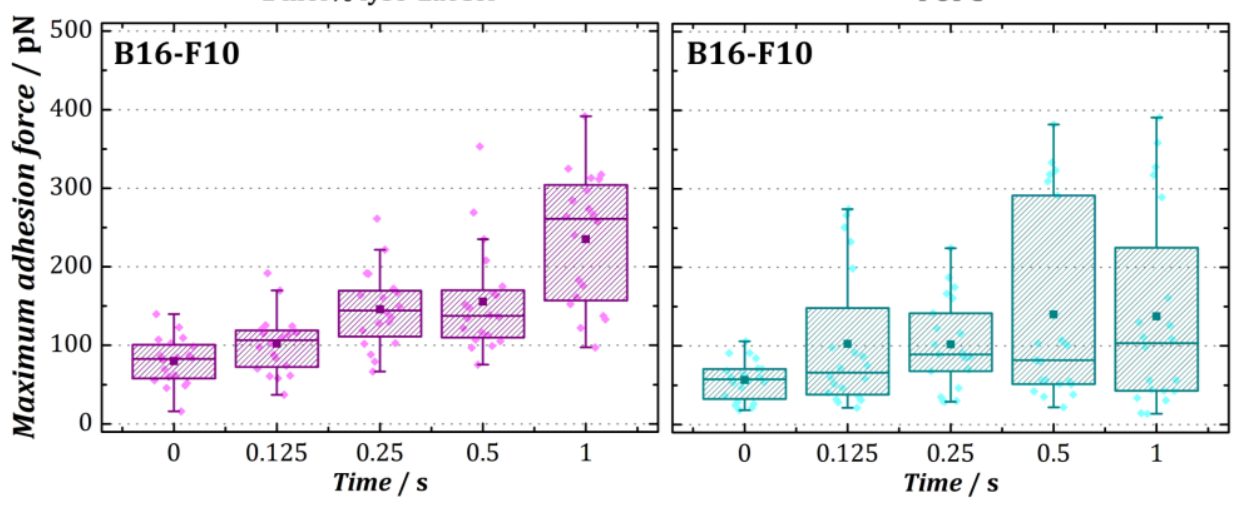

[D]

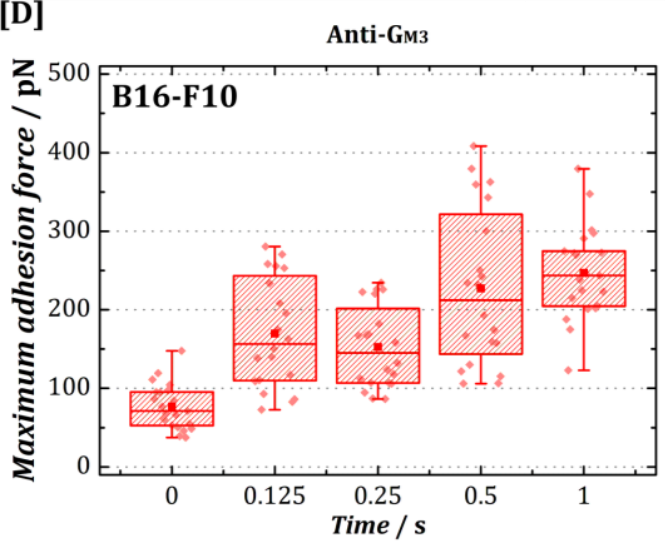

[E]

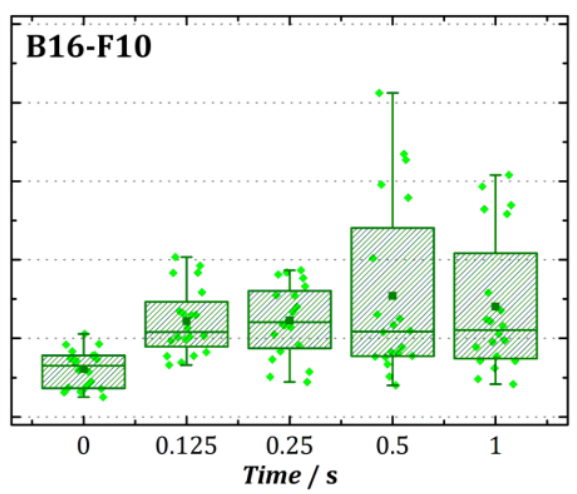

Figure 3.13: Representation of the distribution of the maximum adhesion forces between B16-F10 and B16-F1 cells and the respective substrate obtained at contact times smaller than $1 \mathrm{~s}$ with single-cell force spectroscopy. For the measurement of the $\mathrm{G}_{\mathrm{M} 3}$-lyso-LacCer adhesion forces, untreated single cells were pressed onto SSLMs containing either $10 \mathrm{~mol} \%$ [A, $\left.\mathrm{A}^{\prime}\right]$ or $1 \mathrm{~mol} \%$ [B] of the binding partner lyso-LacCer. To prove that the cell adhesion was initiated by the interaction between $\mathrm{G}_{\mathrm{M} 3}$ and lyso-LacCer, control measurements were made. For pure POPC membranes [C], no binding partners for $\mathrm{G}_{\mathrm{M} 3}$ were available. In further control measurements, the individual cells were first treated with a specific anti-Gм3 antibody [D] or the enzyme sialidase [E] and pressed on 10 mol\% lysoLacCer-containing SSLMs. Here, $50 \%$ of all data points are represented by a box containing the median (solid line) and the mean (illustrated by squares in the respective color). The box is limited by an upper (75 \% threshold) and lower ( $25 \%$ threshold) quartile. Data points outside of the box, whose values are limited to 1.5 times the box length ${ }^{177}$, are described by whiskers. Values, which are not within the whisker range, are referred to as outliers. Measurements include about 5 cells and 15-25 force curves per category. 
[A]

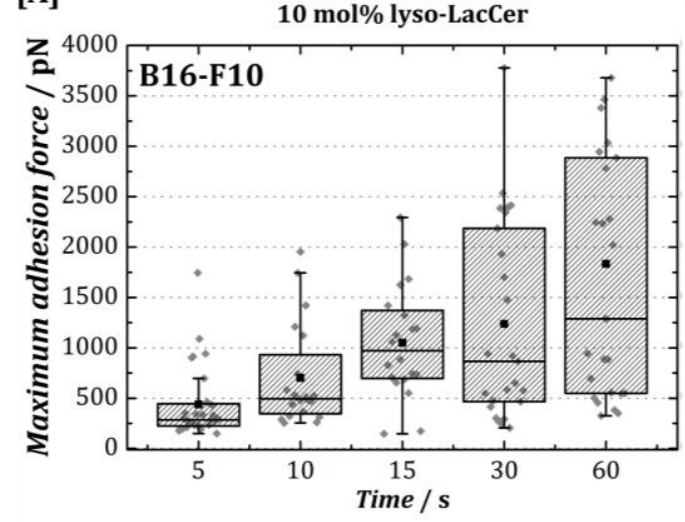

[B]

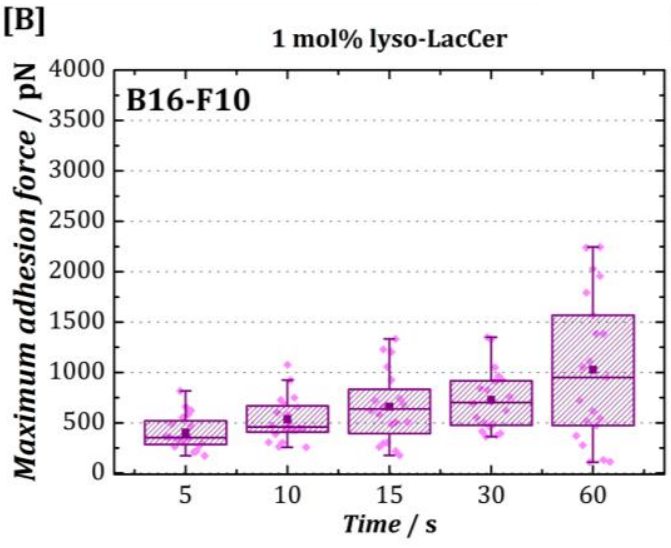

[D]

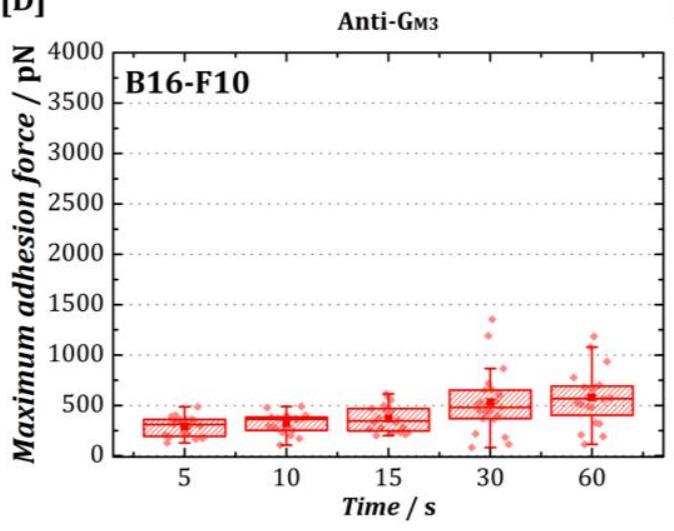

$\left[A^{\prime}\right]$

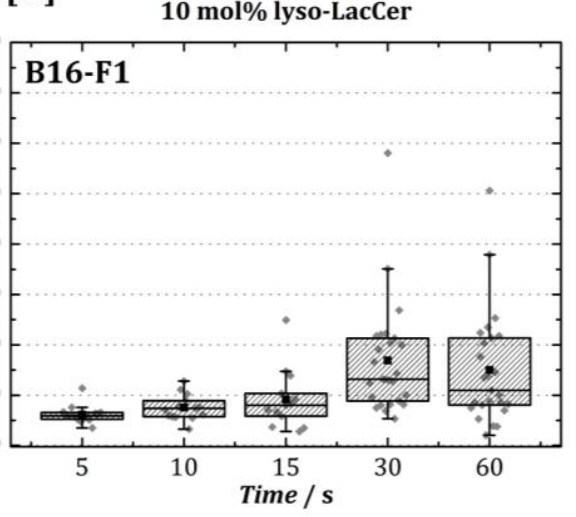

[C]

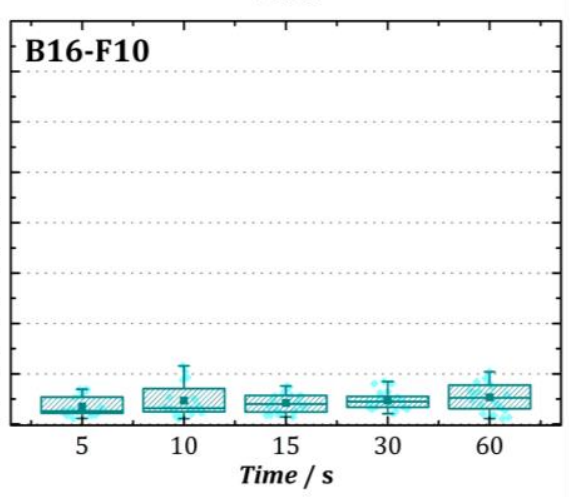

[E]

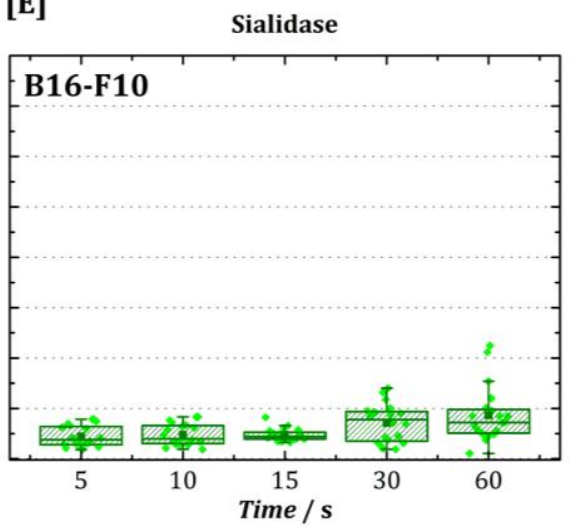

Figure 3.14: Representation of the maximum adhesion forces between B16-F10 and B16-F1 cells and the respective substrate obtained at contact times ranging from 5-60 s with single-cell force spectroscopy. As already described in Figure 3.13, the interaction between Gм3 and its binding partner lyso-LacCer is represented by the contact of untreated single cells onto SSLMs containing either $10 \mathrm{~mol} \%\left[\mathrm{~A}, \mathrm{~A}^{\prime}\right]$ or $1 \mathrm{~mol} \%$ [B] of lyso-LacCer. For control measurements, untreated cells were brought in contact with pure POPC membranes [C] to ensure that no binding partners were available to $\mathrm{G}_{\mathrm{M} 3}$. For further control measurements, the individual cells were first treated with a specific anti-G $\mathrm{G}_{\mathrm{M} 3}$ antibody [D] and the enzyme sialidase [E] and pressed on $10 \mathrm{~mol} \%$ lyso-LacCer-containing SSLMs. Here, $50 \%$ of all data points are represented by a box containing the median (solid line) and the mean (illustrated by squares in the respective color). The box is limited by an upper (75\% threshold) and lower (25\% threshold) quartile. Data points outside of the box, whose values are limited to 1.5 times the box length ${ }^{177}$, are described by whiskers. Values, which are not within the whisker range, are referred to as outliers. Measurements include about 5 cells and 15-25 force curves per category. 
In general, it can be stated that longer contact times led to higher adhesion strengths in carbohydrate-based measurements. For control experiments, no similarly pronounced trend was observed. At contact times smaller than $1 \mathrm{~s}$, mean maximum adhesion forces between $140-250 \mathrm{pN}$ were found for the interaction between cells and $10 \mathrm{~mol} \%$ lyso-LacCer-containing membranes (cf., Figure 3.13 [A]), while mean maximum adhesion forces between cells and 1 mol\% lyso-LacCer-doted membranes (cf. Figure 3.13 [B]) showed slightly decreased mean force values between 80$230 \mathrm{pN}$. This result is plausible when the cell attachment is supported by an interplay of $\mathrm{G}_{\mathrm{M} 3}$ with its binding partner lyso-LacCer, since less binding partners led to weaker adhesion. For the control measurements, mean maximum adhesion forces between $60-140 \mathrm{pN}$ were found in the case of POPC (cf. Figure 3.13 [C]), while for anti-Gм3 antibody (cf. Figure 3.13 [D]) mean force values of $80-240 \mathrm{pN}$ and for sialidase (cf. Figure 3.13 [E]) mean force values between $60-140 \mathrm{pN}$ were observed for contact times smaller than $1 \mathrm{~s}$. Based on these data, it can be proposed that a stronger adhesion of the B16-F10 cells only occurs when an intact interaction between GM3 and lyso-LacCer is present. The finding that the mean maximum force values of the antiGM3 antibody measurements are similar to those of the carbohydrate-based interactions can be explained by the fact that the antibody might not block all Gм3 molecules on the cellular plasma membrane, and thus still a few binding partners for lyso-LacCer were available. Besides, a reversible binding of the antibody is reasonable.

Within the next section, the maximum adhesion forces obtained at contact times smaller than $1 \mathrm{~s}$ are compared to maximum adhesion forces detected at contact times between 5-60 s. In all control measurements only mean maximum adhesion forces between 180-270 pN (POPC, cf. Figure 3.14 [C]), 290-570 pN (anti-GM3, cf. Figure 3.14 [D]), and 220-430 pN (sialidase, cf. Figure 3.14 [E]) were observed. These forces were only slightly larger than those obtained at contact times smaller than $1 \mathrm{~s}$. Significantly increased mean maximum forces between $440-1800 \mathrm{pN}$ were found in the case of 10 mol\% lyso-LacCer (cf. Figure 3.14 [A]). Even when the lyso-LacCer concentration is reduced to $1 \mathrm{~mol} \%$, mean maximum adhesion forces between $400-$ $1000 \mathrm{pN}$ (cf. Figure 3.14 [B]) were obtained, which also differed significantly from the mean maximum adhesion forces that occurred within the control measurements. 
Same SCFS studies with the weakly metastatic and thus less metastatic B16-F1 cancer cell showed a comparable correlation between contact times and mean maximum adhesion forces (cf. Figure 6.4 and Figure 6.5, Chapter 6). At contact times smaller than $1 \mathrm{~s}$, the mean maximum adhesion force values of $80-200 \mathrm{pN}(10 \mathrm{~mol} \%$ lysoLacCer, cf. Figure $6.4[\mathrm{~A}]$ ) or $60-160 \mathrm{pN}$ (1 mol\% lyso-LacCer, cf. Figure 6.4 [B]) originated from the interaction between B16-F1 cells and lyso-LacCer-doped membranes. Same studies with the B16-F10 cell line showed comparable mean maximum adhesion forces. Mean maximum adhesion force values of $60-160 \mathrm{pN}$ were obtained when using an anti-Gм3 antibody (cf. Figure 6.4 [D]). Studies examining the mean maximum adhesion forces with pure POPC membranes (cf. Figure $6.4[\mathrm{C}]$ ) revealed values ranging from 90-200 pN. Only the control sample with the enzyme sialidase showed increased mean maximum adhesion forces between $140-280 \mathrm{pN}$ (cf. Figure 6.4 [E]). The fact that the treatment with the enzyme sialidase caused higher maximum adhesion forces of the B16-F1 cells compared to the F10 variant is a rather unexpected result, since the enzyme is known to cleave off the sialic acid residue of ganglioside molecules. Thus, no or at least less binding partners should be available for lyso-LacCer, which in turn should lead to lower maximum adhesion forces. Since all maximum adhesion forces for sialidase treatment were obtained from one measurement day, it could be that the B16-F1 cells were altered on this day or that the enzyme revealed less efficiency, which might have provoked various mechanisms in the cells leading to a stronger attachment than usual.

At contact times longer than $5 \mathrm{~s}$, increased adhesion forces also occurred for the B16F1 cells. At contact times between 5-60 s, the mean maximum adhesion forces of the interaction between B16-F1 cells and lyso-LacCer-doped lipid membranes range between $300-750 \mathrm{pN}$ (10 mol\% lyso-LacCer, cf. Figure 6.5 [A]) or $250-690 \mathrm{pN}$ (1 mol\% lyso-LacCer, cf. Figure $6.5[\mathrm{~B}]$ ). Thus, with the exception of the sialidase measurement (480-1000 pN, cf. Figure 6.5 [E]), those mean maximum adhesion forces were higher than the mean maximum adhesion force values detected for the interaction with pure POPC membranes (280-420 pN, cf. Figure 6.5 [C]) and for anti$\mathrm{G}_{\mathrm{M} 3}$ measurements (220-500 pN, cf. Figure 6.5 [D]).

The performed SCFS experiments showed that the adhesion of both cell lines was only enhanced if both GSLs were in close proximity to each other. If this interaction was 
inhibited or if one of the two binding partners was missing, significantly weaker maximum adhesion forces were determined. Interestingly, largest differences to control measurements were found for long contact times indicating active processes inside the cells, which strengthened the adhesion to the underlying substrate.

The fact that the B16-F1 cell line exhibits weaker maximum adhesion forces to the lyso-LacCer-doped membranes could either indicate that the B16-F10 cells provide more $\mathrm{G}_{\mathrm{M} 3}$ on their surfaces or that the more metastatic cell line is able to recruit the surface $\mathrm{G}_{\mathrm{M} 3}$ more rapidly to the basal adhesion site through its increased metabolism or cell cycle. Since it was not possible to prove an enhanced $\mathrm{G}_{\mathrm{M} 3}$ expression on the surface of the B16 F10 cell line either by immunostaining or cytometry experiments (cf. Chapter 3.1), it again implies that stronger adhesion does not necessarily result from the total amount of $\mathrm{G}_{\mathrm{M} 3}$ on the cell surface, but rather depend on the fact that the invasive B16-F10 cells is able to recruit adhesion molecules, such as $\mathrm{G}_{\mathrm{M} 3}$, faster and more efficiently to the basal membrane.

Since strong maximum adhesion forces were observed during the adhesion of B16 cells to $10 \mathrm{~mol} \%$ lyso-LacCer, it is of great interest to examine how the interaction between carbohydrate moieties contribute to the maximum adhesion forces and to check if internal processes of the cells contributes to initial adhesion. Therefore, SCFS results were put into context with colloidal probe microscopy (CPM) studies (cf. Chapter 3.5) and jointly discussed with the ongoing literature in the Chapter 3.6.

\subsection{FORCE SPECTROSCOPY OF MODEL MEMBRANES}

\subsubsection{QUANTIFICATION AND VALIDATION OF THE CARBOHYDRATE-CARBOHYDRATE INTERACTION}

Within Chapter 3.3, the preparation of solid supported lipid membranes (SSLMs) including glycosphingolipids (GSLs) and the possible formation of GSL-enriched microdomains (GEM) were discussed. Due to the advantage of a known composition and the opportunity to exclude external influences by biochemical pathways, model membrane studies offer the great possibility to quantify the specific interaction between particular molecule moieties influencing cellular attachment. This specificity 
is difficult to achieve within cell experiments due to the wide variety of different molecules within the cell cortex regulating adhesion and motility. To examine the $\mathrm{Ca}^{2+-}$ dependent binding strength between $\mathrm{G}_{\mathrm{M} 3}$ and lyso-LacCer, the GSLs were incorporated into solid supported lipid membranes to mimic the plasma membrane of mammalian cells. In this thesis, the GM3-containing SSLMs represented the plasma membrane of the carcinoma mouse cell lines B16-F1 and B16-F10, whereas the incorporated lyso-LacCer functioned as a binding partner for $\mathrm{G}_{\mathrm{M} 3}$. The interaction between those GSLs was analyzed by means of colloidal probe microscopy (CPM), an approach based on atomic force microscopy (AFM). ${ }^{208-210}$
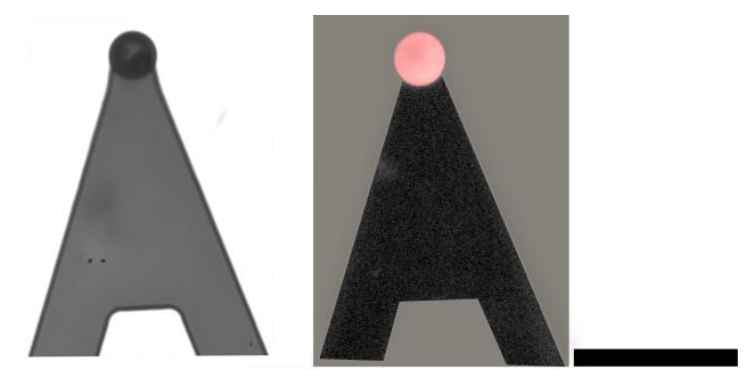

Figure 3.15: Image of a borosilicate glass microsphere glued to a cantilever tip (left). For colloidal probe microscopy experiments, the microsphere was coated with a Texas Red ${ }^{\mathrm{TM}}$ DHPE labeled 10 mol\% $\mathrm{G}_{\mathrm{M} 3}$-containing lipid membrane (right) for $15 \mathrm{~min}$. Scale bar: $50 \mu \mathrm{m}$.

SCFS (cf. Chapter 3.4) already revealed strong maximum adhesion forces between B16-F1 and B16-F10 cells and lyso-LacCer containing lipid membranes. CPM studies were applied to verify how the specific interplay between GM3 and lyso-LacCer contributes to the maximum adhesion forces obtained by SCFS. Based on this, the impact of carbohydrate-carbohydrate interactions on the initial adhesion processes of B16 cells can be discussed. For CPM, a glass microsphere coated with a $10 \mathrm{~mol} \%$ GM3-doted lipid membrane (cf. Figure 3.15) was brought into contact with lysoLacCer-containing or a pure POPC solid supported lipid membranes. The influence of the membrane composition was examined by reducing the lyso-LacCer concentration from $10 \mathrm{~mol} \%$ to $1 \mathrm{~mol} \%$. For control measurements, the B16 cells were brought into contact with pure POPC membranes to rule out the possibility that the maximum adhesion forces obtained within CPM arose from unspecific binding forces, which do not depend on carbohydrates involved in this system. Additionally, the influence of $\mathrm{Ca}^{2+}$ ions was studied by analyzing the maximum interaction forces between GM3 and lyso-LacCer using a $\mathrm{Ca}^{2+}$ - or EDTA-containing measurement-buffer (cf. Table 2.2, Chapter 2.2.3). 
In order to investigate the specific binding affinity between $\mathrm{G}_{\mathrm{M} 3}$ and lyso-LacCer, the maximum adhesion forces between the glycosphingolipid moieties were examined by collecting force-distance curves on model systems (cf. Figure 3.16) that were analyzed in the same manner as for SCFS experiments (cf. Chapter 3.4). Again, the binding strength and possible unbinding processes were studied using various contact times ranging from 0-60 s. The distribution of the individual maximum adhesion forces for each contact time is shown in Figure 3.17. Figure 3.16 [A] demonstrates exemplary forces-distance curves recorded at contact times of $0 \mathrm{~s}$ (violet), $1 \mathrm{~s}$ (blue) $5 \mathrm{~s}$ (green) and $60 \mathrm{~s}$ (orange) in the presence of $\mathrm{Ca}^{2+}$ ions, illustrating the maximum adhesion forces between $\mathrm{G}_{\mathrm{M} 3}$ and lyso-LacCer, both embedded in lipid membranes. Although less pronounced compared to SCFS studies (cf. Chapter 3.4), Figure 3.16 [A] shows that longer contact times led to stronger adhesion forces between $\mathrm{G}_{\mathrm{M} 3}$ - and lyso-LacCer containing membranes. In Figure 3.16 [B], exemplary force-distance curves of control measurements (light blue and light orange) were compared to force curves representing the Gмз-lysoLac interaction (blue and orange) at contact times of $1 \mathrm{~s}$ and $60 \mathrm{~s}$. In presence of $\mathrm{Ca}^{2+}$ ions, the forcedistance curve of the control measurements demonstrated measurable maximum adhesion forces between a GM3-doted and a pure POPC membrane. However, even at a contact time of $60 \mathrm{~s}$, the obtained adhesion force was significantly lower than the maximum adhesion force revealed by the force curve representing the interaction between $\mathrm{G}_{\mathrm{M} 3}$ and its binding partner lyso-LacCer. These findings indicate that the interaction with only the matrix lipid POPC is negligible. 
[A]

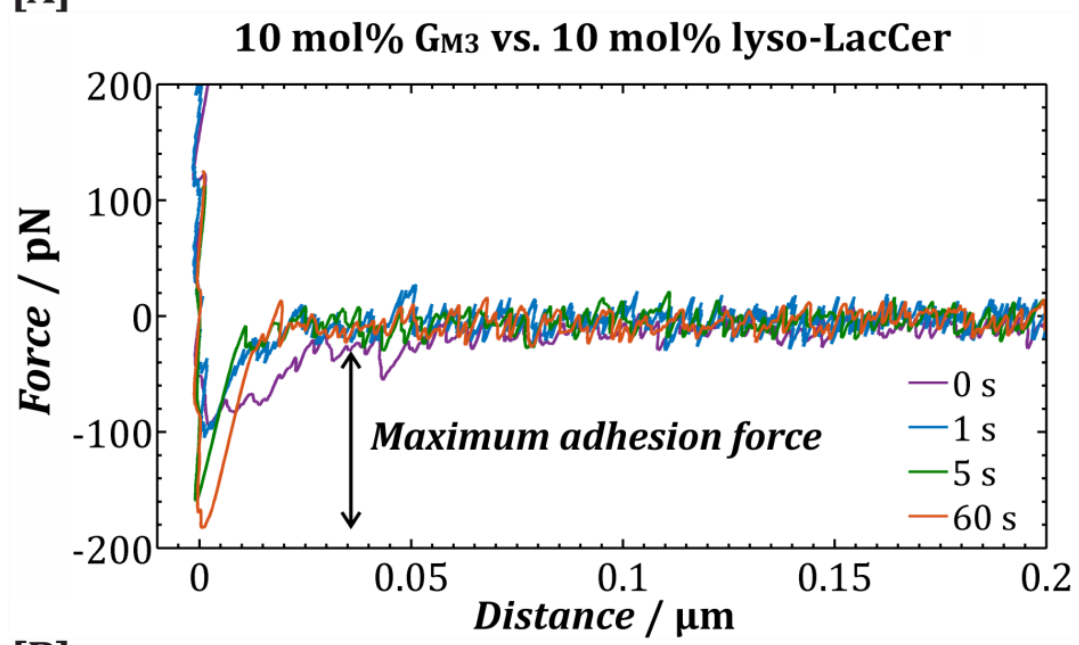

[B]

Control

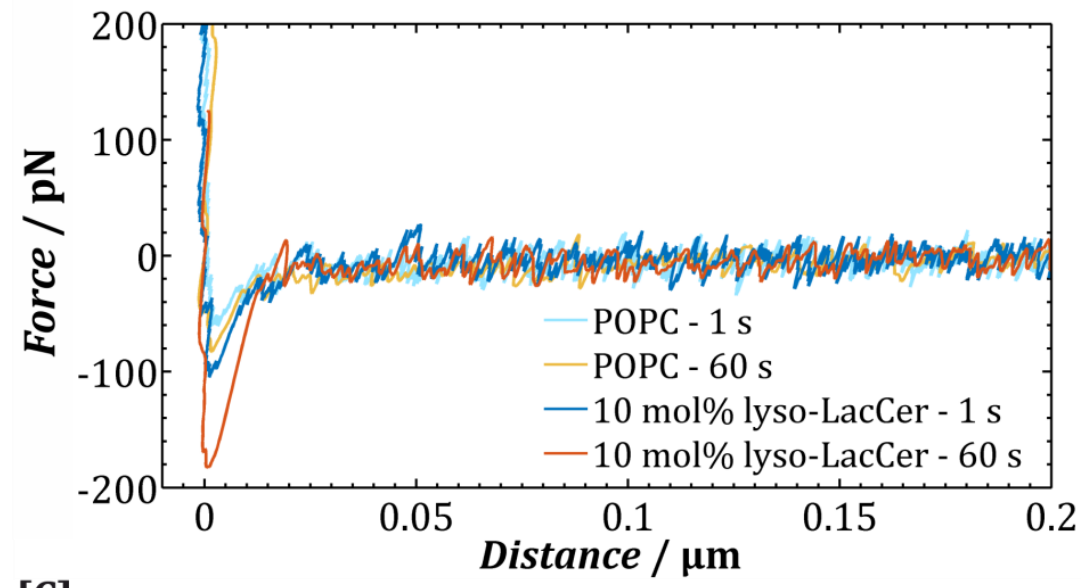

[C]

10 mol\% GM3 vs. 10 mol\% lyso-LacCer

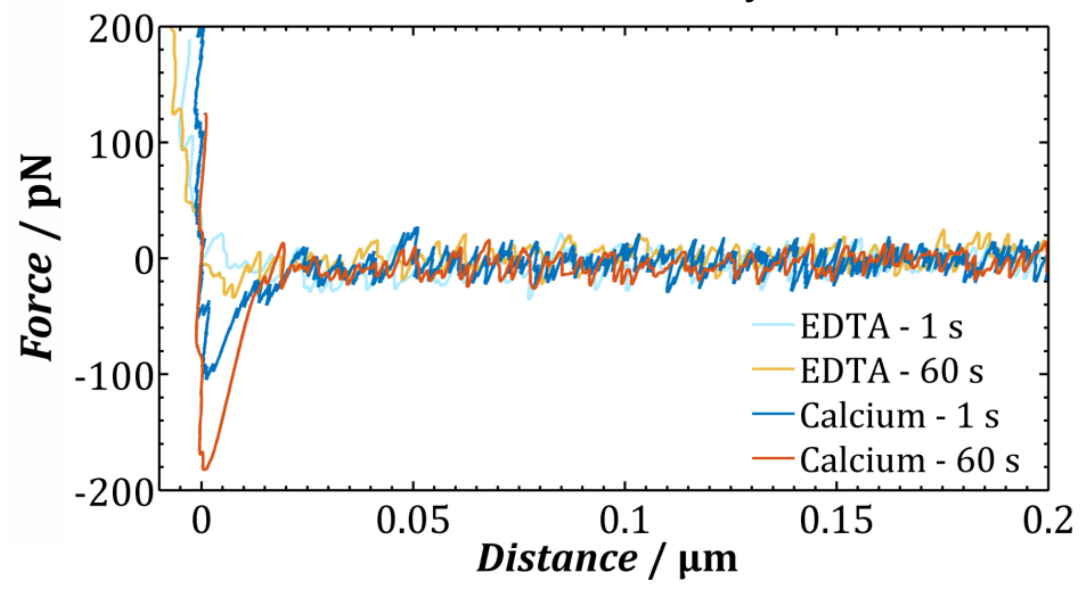

Figure 3.16: Exemplary force-distance curves of the retraction process recorded by colloidal probe microscopy demonstrating the maximum adhesion forces between $10 \mathrm{~mol} \% \mathrm{G}_{\mathrm{M} 3}-$ and $10 \mathrm{~mol} \%$ lyso-LacCer-doted or pure POPC lipid membranes. In [A] typical force-distance curves are shown presenting the binding strength of $\mathrm{G}_{\mathrm{M} 3}$ and lyso-LacCer after various contact times of $0 \mathrm{~s}$ (violet), $1 \mathrm{~s}$ (blue), $5 \mathrm{~s}$ (green) and $60 \mathrm{~s}$ (orange) in the presence of $\mathrm{Ca}^{2+}$ ions. In [B], the maximum adhesion forces of the $\mathrm{G}_{\mathrm{M} 3}$-lyso-LacCer interaction (blue and orange) are compared to the maximum adhesion forces between $\mathrm{G}_{\mathrm{M} 3}$ and POPC (light blue and light orange) detected at contact times of $1 \mathrm{~s}$ and $60 \mathrm{~s}$, again in the presence of $\mathrm{Ca}^{2+}$ ions. The force-distance curves plotted in [C] display the influence of $\mathrm{Ca}^{2+}$ ions on the maximum adhesion forces between $\mathrm{G}_{\mathrm{M} 3}$ and lyso-LacCer. 
[A]

$10 \mathrm{~mol} \%$ lyso-LacCer

$1 \mathrm{~mol} \%$ lyso-LacCer

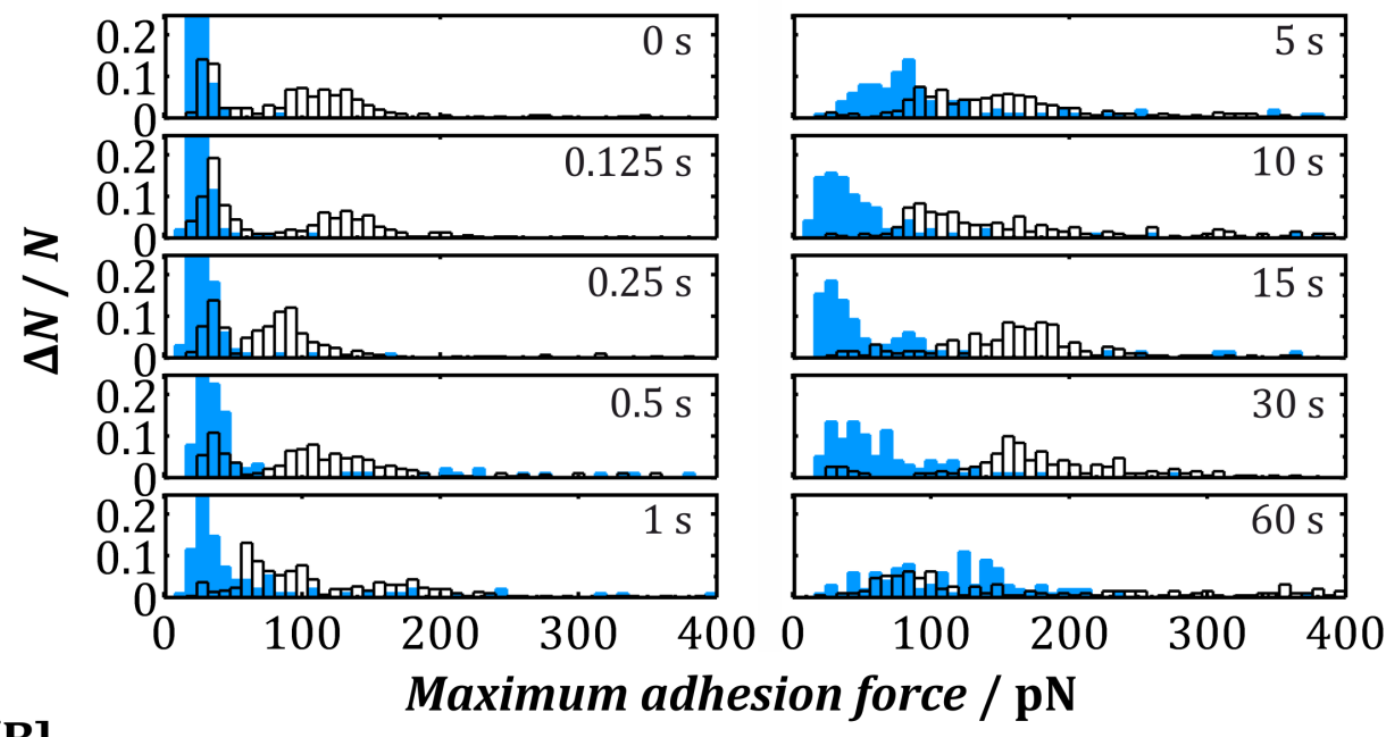

[B]

$10 \mathrm{~mol} \%$ lyso-LacCer
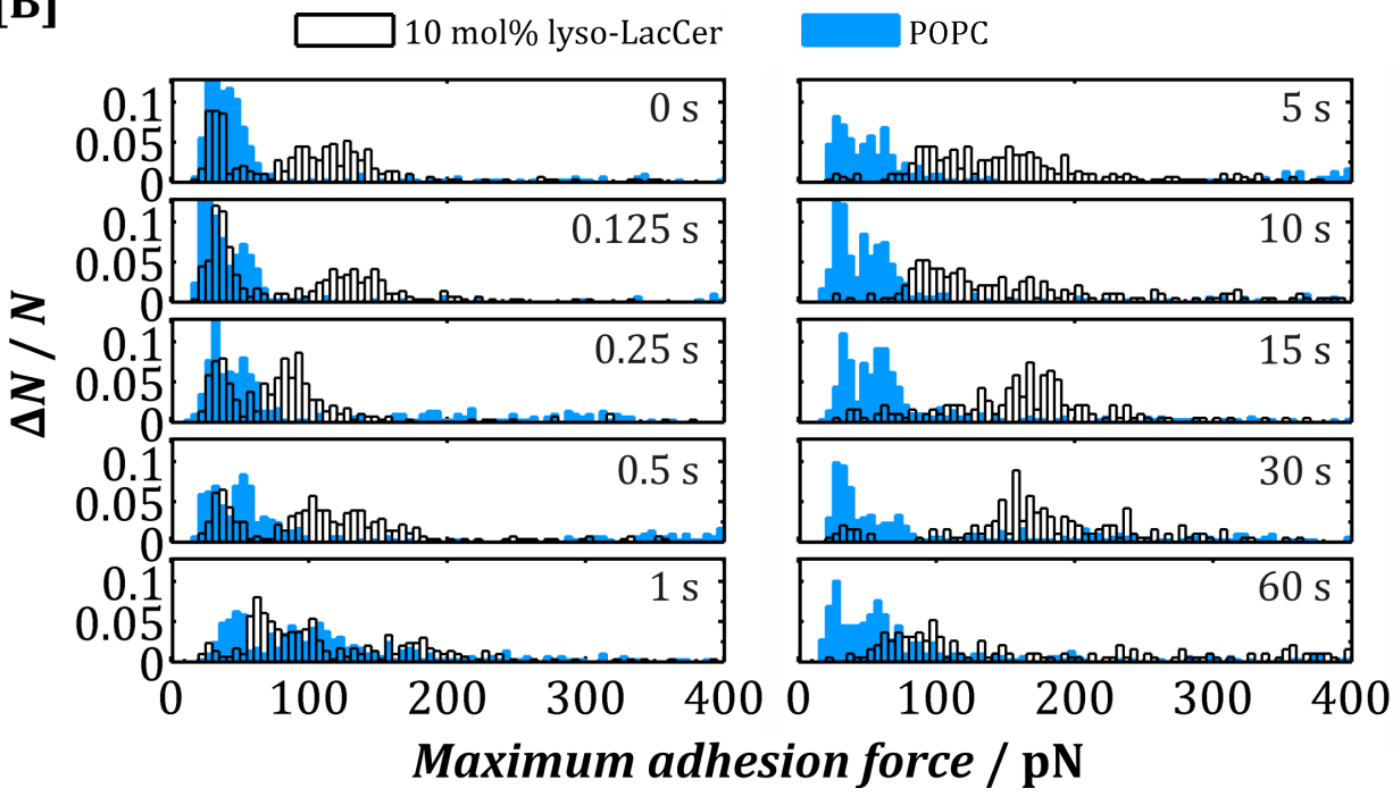

Figure 3.17: Histograms of maximum adhesion forces obtained by colloidal probe microscopy measurements. [A] In order to examine the adhesion forces between the glycosphingolipids $\mathrm{G}_{\mathrm{M} 3}$ and lyso-LacCer, a $10 \mathrm{~mol} \% \mathrm{G}_{\mathrm{M} 3}-$ containing lipid membrane was spread onto a glass microsphere and brought into contact with an underlying $10 \mathrm{~mol} \%$ (transparent histograms) or $1 \mathrm{~mol} \%$ (blue histograms) lyso-LacCer embedded lipid membrane. [B] For control measurements, the forces obtained between $10 \mathrm{~mol} \% \mathrm{G}_{\mathrm{M} 3}$ and $10 \mathrm{~mol} \%$ lyso-LacCer (transparent histograms) were compared with the maximum adhesion forces that occurred between a $10 \mathrm{~mol} \% \mathrm{G}$ м-containing and a pure POPC lipid membrane (blue histograms). Measurements include 188-298 force curves for 10 mol lysoLacCer, 65-100 force curves for 1 mol lyso-LacCer and 272-294 force curves for POPC.

It was interesting to observe that in absence of $\mathrm{Ca}^{2+}$ ions (EDTA, cf. Figure $3.16[\mathrm{C}]$ ) no measurable maximum adhesion forces were detected between $\mathrm{G}_{\mathrm{M} 3}$ and lyso-LacCer indicating once more the importance of $\mathrm{Ca}^{2+}$ ions for an efficient formation of carbohydrate-carbohydrate interaction.

While many typical unbinding processes such as jumplike rupture steps ${ }^{135,136}$ or tetherlike steps ${ }^{135,136}$ were observed in recorded force-distance curves within SCFS 
studies (cf. Figure 3.11), such processes are rarely detectable in the force-distance curves collected by CPM studies (cf. Figure 3.16). Even at high contact times of $60 \mathrm{~s}$, if at all, only tetherlike steps were observable indicating strong adhesion forces between $\mathrm{GM}_{\mathrm{M}}$ - and lyso-containing lipid membranes possibly accumulated into GEMs.

Compared to the maximum adhesion forces obtained within SCFS, a rather broad distribution of measured maximum adhesion forces was detectable within CPM. Also, a significant amount of outliers towards values higher than $1000 \mathrm{pN}$ was observed, which is surprising considering that the carbohydrate-carbohydrate interaction are assumed to be weak. In addition, it was interesting to observe that the maximum adhesion forces obtained between 10 mol\% GM3- and 10 mol\% lyso-LacCer-doted membranes (transparent histograms, cf. Figure 3.17 [A]) showed a bimodal distribution in contrast to the approximately normal distribution of the maximum adhesion forces detected between $10 \mathrm{~mol} \% \mathrm{G}_{\mathrm{M} 3}$ and $1 \mathrm{~mol} \%$ lyso-LacCer (blue histograms, cf. Figure 3.17 [A]) or 10 mol\% GM3 and POPC (blue histograms, cf. Figure $3.17[\mathrm{~B}])$. Moreover, the appearance of the two maxima at $50-100 \mathrm{pN}$ and 100-250 pN seemed to be related not only to the concentration of the incorporated binding partners, but also to the duration of the contact time. Thus, within the interaction of $10 \mathrm{~mol} \% \mathrm{G}_{\mathrm{M} 3}$ and $10 \mathrm{~mol} \%$ lyso-LacCer, both maxima appeared very clearly at contact times smaller than $1 \mathrm{~s}$. Here, maximum adhesion forces between 50-100 pN characterize the first maximum, whereas the second maximum reveals maximum adhesion forces ranging from 100-250 pN. With increasing contact times from 5-60 s between both GSL-containing membranes, a stronger adhesion was observed resulting in maximum adhesion forces of $100-400 \mathrm{pN}$. Interestingly, the height of the first maximum appears to decrease within contact times smaller than $1 \mathrm{~s}$ and is only barely observable at higher contact times ranging from 5-60 s.

A plausible explanation for a bimodal distribution could be the presence of GSL enriched microdomains (GEMs), which were possibly detected in topography studies of $10 \mathrm{~mol} \% \mathrm{GM}_{\mathrm{M} 3}$ and $10 \mathrm{~mol} \%$ lyso-LacCer-doted membrane surfaces using atomic force microscopy (cf. Figure 3.9, Chapter 3.3). Supposing that GSLs accumulate in GEMs, and thus are not homogeneously distributed in the membrane, it is always possible to analyze areas of the neighboring membranes, which do contain GSLs and those regions, which do not contain GSLs. As shown in the blue-colored histograms in 
Figure $3.17[\mathrm{~B}]$, the interaction between $\mathrm{G}_{\mathrm{M} 3}$-doted and pure POPC membranes provided maximum adhesion forces between $50-100 \mathrm{pN}$ at contact times from 0-60 s, which corresponded well to the obtained first maxima representing the maximum adhesion forces between 10 mol\% Gм3 and 10 mol\% lyso-LacCer at contact times smaller than $1 \mathrm{~s}$. Since the maximum adhesion forces between GM3 and POPC could not be increased using longer contact times (with the exception of single measurements), these findings support the assumption that the first maximum observed within the interaction between $10 \mathrm{~mol} \% \mathrm{Gm} 3$ and $10 \mathrm{~mol} \%$ lyso-LacCer is due to nonspecific interactions caused by GSL-POPC interactions. This in turn verifies that high maximum adhesion forces ranging from $100-400 \mathrm{pN}$ at contact times of 0-60 s were exclusively caused by an interaction between $10 \mathrm{~mol} \% \mathrm{Gm}$ and $10 \mathrm{~mol} \%$ lyso-LacCer, since no comparable maximum adhesion forces were observed within control experiments at contact times greater than $1 \mathrm{~s}$.

The phenomenon that the first maximum of the bimodal distribution almost disappeared with longer contact times assumes that POPC lipids were steadily replaced by GSLs diffusing into the contact area of neighboring GSL-doted lipid membranes, resulting in a reduction of nonspecific $\mathrm{G}_{\mathrm{M} 3}-\mathrm{POPC}$ interactions. Although less pronounced, the interaction between $10 \mathrm{~mol} \% \mathrm{G}_{\mathrm{M} 3}$ and $1 \mathrm{~mol} \%$ lyso-LacCer (blue histograms, cf. Figure 3.17 [B] also showed a reduction of the maximum adhesion forces between 50-100 pN with longer contact times. However, similar to the $\mathrm{GM}_{\mathrm{M}}$ POPC interaction, maximum adhesion forces of $50-150 \mathrm{pN}$ were detected even at contact times of $60 \mathrm{~s}$, indicating that several GSLs need to be involved to create relatively strong bonds between two adjacent GSL-doped membranes to enhance adhesion.

It was striking to observe that the interaction between $10 \mathrm{~mol} \%$ GM3- and $10 \mathrm{~mol} \%$ lyso-LacCer-containing SSLMs exposed relatively high maximum adhesion forces ranging from 100-400 pN at contact times of 0-60 s. Those forces corresponded well with the maximum adhesion forces obtained at contact times smaller than $5 \mathrm{~s}$ within SCFS. Large adhesion forces, measured with SCSF at long contact times were not detected in CPM, indicating that the interaction between $\mathrm{G}_{\mathrm{M} 3}$ and lyso-LacCer contributes to the B16 cell adhesion at least at contact times smaller than $1 \mathrm{~s}$. In Chapter 3.6, these findings and all SCFS and CPM results are discussed together with 
the ongoing literature to achieve an integrated view about the impact of carbohydrate-carbohydrate interactions on the cellular attachment.

\subsection{CARBOHYDRATE-CARBOHYDRATE ADHESION FORCES}

The general aim of this work was to determine the impact of the interaction between Gм3 and lyso-LacCer on the adhesion of the B16-F1 and B16-F10 melanoma cancer cells. In addition to studying the distribution and organization of the GSLs in cellular and model membranes, single-cell force spectroscopy (SCFS, cf. Chapter 3.4) as well as colloidal-probe microcopy (CPM, cf. Chapter 3.5) experiments were performed allowing quantitative analysis of adhesion forces. SCFS was used to investigate the maximum adhesion forces between living $\mathrm{G}_{\mathrm{M} 3}$-expressing B16 cells and lyso-LacCer, whereas CPM was used to determine the maximum adhesion forces between $\mathrm{G}_{\mathrm{M} 3}$ and lyso-LacCer directly. Since CPM is based on model membranes, it offers the great advantage of ensuring full control over molecular compositions and excludes interference with other molecules such as integrins. Also, the impact of internal cellular processes on adhesion forces can be excluded and the observed adhesion forces can be solely attributed to the interaction between $\mathrm{G}_{\mathrm{M} 3}$ and lyso-LacCer.

Depending on the degree of GEM formation, carbohydrate-carbohydrate interactions (CCIs) are known to have association constants of about $\sim 10^{3}-10^{8} \mathrm{M}^{-1}$, which are below the association constants of carbohydrate-protein (CPIs, $\sim 10^{8} \mathrm{M}^{-1}$ ) and protein-protein interactions (PPIs, $\sim 10^{8}-10^{9} \mathrm{M}^{-1}$ ), ${ }^{58,207,211}$ and therefore long time considered to be too weak to mediate cellular attachment. However, our results showed that indeed strong adhesion forces exist between $\mathrm{G}_{\mathrm{M} 3}$ and lyso-LacCer, most likely due to the interaction of many bonds in parallel. Using CPM, maximum adhesion forces of 100-400 pN (cf. Figure 3.17) at contact times of 0-60 s were detected between $10 \mathrm{~mol} \% \mathrm{G}_{\mathrm{M} 3}$ and $10 \mathrm{~mol} \%$ lyso-LacCer, which were quite similar to adhesion forces measured between $200 \mathrm{kDa}$ glycan molecules $(200-300 \mathrm{pN})^{45}$ and mean adhesion forces obtained between Lewis ${ }^{X}$ moieties $(90-230 \mathrm{pN}){ }^{208}$ The maximum adhesion forces between $\mathrm{G}_{\mathrm{M} 3}$ and lyso-LacCer were even comparable with adhesion forces detected between entire proteoglycan molecules (50-400 pN), protein-glycan (CPI, P-selectin/carbohydrate ligand, $100-300 \mathrm{pN}$ ) and single 
antibody-antigen interactions (PPI, human serum albumin (HSA)/anti-HSA antibody), where rupture forces of $\sim 240 \mathrm{pN}$ were detected. ${ }^{212-214}$ Considering that single Gмз-lyso-LacCer interactions might rupture at approximately 40-60 pN (cf. Figure 6.3, Chapter 6), several multivalent GSLs bonds need to be established to ensure short-termed recognition processes by rapid and cooperative interactions to enhance cell adhesion.

To clarify the role of CCI on initial attachment processes of cells, CPM studies were compared with SCFS. Interestingly, the maximum adhesion forces obtained within CPM (100-400 pN, cf. Figure 3.17) were quite similar to maximum adhesion forces observed using SCFS at contact times shorter than $5 \mathrm{~s}(\sim 100-500 \mathrm{pN}$, cf. Figure 3.12). These finding is probably due to the choice of bead size in CPM providing a similar amount of binding partners in the contact area. At contact times of 10-60 s, maximum adhesion forces up to $750 \mathrm{pN}$ for the less invasive B16-F1 cell line and even $1800 \mathrm{pN}$ for the highly metastatic F10 were detected by SCFS (cf. Figure 3.12). These results indicate that the interaction between $\mathrm{G}_{\mathrm{M} 3}$ and lyso-LacCer is able to promote the initial cellular adhesion of the carcinoma B16 cells at least within the first seconds. Interestingly, $\mathrm{G}_{\mathrm{M} 3}$ is known to interact with focal adhesions ${ }^{26}$, integrin receptors ${ }^{62}$ and signaling transducer molecules. ${ }^{25}$ With regard to the very high maximum adhesion forces obtained within SCFS at longer contact times, an association of GM3 with those mentioned adhesive and signaling molecules might induce active internal cellular processes, which in turn lead to an enhanced cell adhesion with adhesion forces in the upper $\mathrm{pN}$ to $\mathrm{nN}$ range.

The effect of divalent cations such as $\mathrm{Ca}^{2+-i o n s}$ on CCIs still remains highly debated even though many CCIs rely on the presence of $\mathrm{Ca}^{2+}$-ions. ${ }^{45}$ In our CPM studies, the interaction between $\mathrm{G}_{\mathrm{M} 3}$ and lyso-LacCer showed a clear $\mathrm{Ca}^{2+}$ dependency since no adhesion forces between $\mathrm{G}_{\mathrm{M} 3}$ and lyso-LacCer were observed in absence $\mathrm{Ca}^{2+}$ ions (cf. Figure $3.16[\mathrm{C}])$. It is believed that $\mathrm{Ca}^{2+}$-ions could bridge negatively charged carbohydrate head groups to stabilize the conformation of involved carbohydrate moieties, thus leading to the formation of hydrogen bonds and an interaction between hydrophobic surfaces. ${ }^{45,58,215}$ 
To conclude, we were able to demonstrate that the interaction between GM3 and lysoLacCer have the potential to provide sufficient adhesion strengths to initiate the cellular attachment of the B16 melanoma cancer cells before active cellular processes enhances adhesion. Since no major contact area differences were detected between B16-F1 and B16-F10 cells, we additionally assume that the much higher maximum adhesion forces of the B16-F10 cells in comparison to the F1 variant might result from an enhanced cellular metabolism leading to a more effective recruitment of $\mathrm{G}_{\mathrm{M} 3}$ to the attachment site. 


\section{CONCLUSION

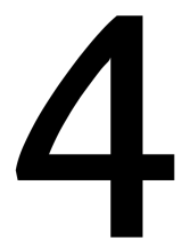

With their highly variable carbohydrate head groups facing the surrounding medium of cells, glycosphingolipids offer vital short-term recognition systems to influence cellular adhesion and signaling processes. To investigate the role of carbohydratecarbohydrate interactions (CCIs) on the cellular attachment of carcinoma cells, two different atomic force microscopy (AFM) based methods, colloidal probe microscopy (CPM) as well as single-cell force spectroscopy (SCFS), were implemented in this thesis. Combined with surface based analytical experiments, those studies assessed and quantified the impact of CCIs between Gмз and lyso-LacCer on the initial adhesion of the murine B16 melanoma cancer cells. The Gм3-expressing B16 cells are characterized by different metastasis behaviors. B16-F1 cells are less invasive, while the B16-F10 variant shows an increase of metastases in surrounding organs. ${ }^{5-7}$ By using those cell lines, conclusions about the impact on CCI related to tumor malignancy can be drawn.

SCFS was used to investigate the maximum adhesive forces of living B16 cells, whereas CPM is based on a model membrane system allowing the determination of the maximum adhesion forces between $\mathrm{G}_{\mathrm{m} 3}$ and lyso-LacCer directly. Model membranes are excellent tools to mimic cellular plasma membranes ensuring full control over the molecular composition of the membranes. By this, SSLMs make it possible to obtain direct feedback of the impact of certain lipids under controlled conditions. ${ }^{10}$

The investigation of the distribution and organization of $\mathrm{G}_{\mathrm{M} 3}$ within the cellular plasma membrane of B16 cells by fluorescence microscopy indicated an accumulation 
of GM3 within defined parts of the apical as well as basal side of B16-F1 and B16-F10 cellular membranes (cf. Chapter 3.1). Those results agreed well with topography studies applied to the model membrane system (cf. Chapter 3.3) revealing that $\mathrm{G}_{\mathrm{M}}$ as well as lyso-LacCer were punctually embedded in the leaflet of the solid supported lipid membranes (SSLMs) rather than being homogenously distributed. The observed accumulation in specific domains was also consistent with several studies proposing the formation of GSL-enriched microdomains (GEMs) in biological and model membranes. ${ }^{32,45}$ Further, we observed that GM3 seemed to be predominantly located at periphery of the cellular adhesion areas. Since RICM results suggested that both cell lines adhered closer to the substrate at the cell borders rather than within the center of the cell, an association of $\mathrm{GM}_{\mathrm{M}}$ to adhesion complexes was proposed. This assumption is consistent with cell adhesion studies, proposing an interaction between $\mathrm{G}_{\mathrm{M} 3}$ and focal adhesions ${ }^{26}$, integrin receptors ${ }^{62}$ as well as signaling transducer molecules ${ }^{25}$, which are crucial for cell adhesion and signal transduction.

Fluorescence-based studies and optical cell adhesion experiments (cf. Chapters 3.1 and 3.2) gave information about the cellular behavior and the distribution of $\mathrm{G}_{\mathrm{M} 3}$ on the plasma membranes of the B16 cells and SSLMs. However, they were not sufficient to provide evidence of the impact of CCI on the initial attachment of the B16-F1 and B16-F10 cancer cell lines related to their malignancy. Therefore, AFM-based SCFS (cf. Chapter 3.4) and CPM studies (cf. Chapter 3.5) were used to quantify the initial adhesion of B16-F1 or B16-F10 cells mediated by the interaction between GM3 and lyso-LacCer.

CPM experiments clearly demonstrated that strong maximum adhesion forces of 100$400 \mathrm{pN}$ at contact times from 0-60 s were only obtained if lyso-LacCer was available as a binding partner for $\mathrm{G}_{\mathrm{M} 3}$ (cf. Figure 3.17 [A]). These findings were confirmed by control experiments based on the interaction between $\mathrm{G}_{\mathrm{M} 3}$ and pure POPC membranes showing unspecific maximum adhesion forces between 50-100 pN at same contact times (cf. Figure 3.17 [B]). Additionally, CPM studies confirm the $\mathrm{Ca}^{2+}$ dependency of CCI as no adhesion forces between $\mathrm{G}_{\mathrm{M} 3}$ and lyso-LacCer were observed in absence $\mathrm{Ca}^{2+}$ ions (cf. Figure $3.16[\mathrm{C}]$ ). 
The difference in B16 adhesion behavior depending on shorter and longer contact times revealed another interesting phenomenon. In comparison to CPM, similar maximum adhesion forces of $100-500 \mathrm{pN}$ were detected by SCFS at contact times shorter than $5 \mathrm{~s}$, while longer contact times of 10-60 s resulted in maximum adhesion forces up to $750 \mathrm{pN}$ for the less metastatic B16-F1 cell line and even $1800 \mathrm{pN}$ for the highly invasive F10 variant (cf. Figure 3.12). Since weaker maximum adhesion forces were detected in control experiments applying pure POPC membranes, an anti-GM3 antibody, or the enzyme sialidase (cf. Figure 3.12), the higher maximum adhesion forces observed within SCFS were attributed to the interaction between the cellsurface expressed Gм3 and lyso-LacCer. Along with these results, we propose that the interaction between $\mathrm{G}_{\mathrm{M} 3}$ and lyso-LacCer is able to promote the initial cellular adhesion of the carcinoma B16 cells within the very first seconds. In addition, an association of $\mathrm{G}_{\mathrm{M} 3}$ with adhesive molecules like focal adhesions ${ }^{26}$, integrin receptors ${ }^{62}$ and signaling transducer molecules ${ }^{25}$ could foster active internal cellular processes enhancing adhesion forces.

Regarding the role of malignancy, SCFS showed that the highly malignant B16-F10 cells are able to form significantly stronger adhesion forces with lyso-LacCer than the less invasive F1 variant (cf. Figure 3.12). Since we were not able to confirm that the B16-F10 cells express more $\mathrm{G}_{\mathrm{M} 3}$ on their cell surfaces, we assume that those invasive cells are able to recruit membrane-bound $\mathrm{G}_{\mathrm{M} 3}$ more efficiently to the attachment site due to an enhanced effective internal mechanism. An actively regulated redistribution of $\mathrm{G}_{\mathrm{M} 3}$ from the entire membrane surface to the basal cell membrane and stronger adhesion to surrounding cells would turn out to be particularly advantageous in the metastasis process, bearing in mind that highly metastatic cancer cells must be able to invade surrounding organs in the presence of a constant bloodstream to form further metastases. The effects of CCI on the adhesion of B16 cells within this project are also in good accordance with studies demonstrating that cells adapt the biosynthesis of $\mathrm{G}_{\mathrm{M} 3}$ according to their role and properties. ${ }^{59,216,217}$ Cells forming strong contacts with surrounding cells expressed a higher proportion of $\mathrm{G}_{\mathrm{M} 3}$ than actively proliferating cells, which rather rapidly catabolized the complex ganglioside $\mathrm{GM}_{\mathrm{M}}$ to the disaccharide lactosylceramide (LacCer). ${ }^{59,218}$ As already described in Chapter 1.5, 
GM3 is known to inhibit the tyrosine kinase of the epidermal growth factor (EGF). Therefore, replicative processes are promoted in $\mathrm{G}_{\mathrm{M} 3}$-reduced proliferating cells. ${ }^{59,218}$

In summary, I was able to demonstrate that CCIs lead to strong maximum adhesion forces due to the cooperative character of GSLs involved. Thus, CCIs have the potential to support the initial adhesion process of the B16 melanoma cells. Further, CCIs based adhesion forces were comparable to the forces obtained within PPI or carbohydrateprotein interactions (CPI) in terms of the order of magnitude. In contrast to the rather low variability of PPIs, CCIs have the significant advantage of allowing rapid assembly and disassembly due to their extremely high flexibility and variability.

Compared to CPM, SCFS in general revealed significantly increased maximum adhesion forces at contact times higher than $10 \mathrm{~s}$. While the migration of the GSLs in model membranes to the surrounding substrate is limited by diffusion, the use of living cells offers the possibility of an active reorganization of GSLs and a subsequent association with adhesive molecules including proteins, whereby cell adhesion can be strengthened. Findings that equally strong maximum adhesion forces were present in CPM as well as SCFS studies at contact times smaller than $5 \mathrm{~s}$ indicate again that the B16 cell line benefits greatly from existing CCI, especially in times frames of 5-60 s. This in turn allows the cells to form initial contacts in order to test for suitable adhesion areas before stable PPI are formed. In addition, the invasive nature of the B16-F10 cell line appeared to be fostered by a more active metabolism by an presumably association with adhesion molecules like integrin, which corresponds to the properties of metastatic cancer cell lines and the biological functions that have been attributed to Gмз. 
(1) Berg, J. M.; Tymoczko, J. L.; Stryer, L.; Häcker, B. Biochemie, Korr. Nachdr. der 6. Aufl.; Spektrum Akad. Verl: Heidelberg, 2011.

(2) Cancer Facts \& Figures 2019; American Cancer Society: Atlanta, GA, 2019.

(3) Deutsche Krebsgesellschaft. Maliges Melanom https://www.krebsgesellschaft.de (accessed Feb 21, 2019).

(4) Koch-Institut, R. Bericht zum Krebsgeschehen in Deutschland 2016.

(5) Fidler, I. J. Selection of Successive Tumour Lines for Metastasis. Nature New Biology 1973, 242, 148-149.

(6) Fidler, I. J. Biological Behavior of Malignant Melanoma Cells Correlated to Their Survival in Vivo. Cancer Research 1975, 218-224.

(7) Bosmann, H. B.; Bieber, G. F.; Brown, A. E.; Case, K. R.; Gersten, D. M.; Kimmerer, T. W.; Lione, A. Biochemical Parameters Correlated with Tumor Cell Implantation. Nature 1973, 246, 487-489.

(8) Poste, G.; Doll, J.; Fidler, I. J. Interactions among Clonal Subpopulations Affect Stability of the Metastatic Phenotype in Polyclonal Populations of B16 Melanoma Cells. Proceedings of the National Academy of Sciences 1981, 78 (10), 6226-6230. https://doi.org/10.1073/pnas.78.10.6226.

(9) Singer, S. J.; Nicolson, G. L. The Fluid Mosaic Model of the Structure of Cell Membranes. Science, New Series 1972, 175 (4023), 720-731.

(10) Richter, R. P.; Bérat, R.; Brisson, A. R. Formation of Solid-Supported Lipid Bilayers: An Integrated View. Langmuir 2006, 22 (8), 3497-3505. https://doi.org/10.1021/la052687c.

(11) Forstner, G. G.; Tanaka, K.; Isselbacher, K. J. Lipid Composition of the Isolated Rat Intestinal Microvillus Membrane. Biochem. J. 1968, 109 (1), 51-59.

(12) Forstner, G. G.; Wherrett, J. R. Plasma Membrane and Mucosal Glycosphingolipids in the Rat Intestine. Biochim. Biophys. Acta 1973, 306 (3), 446-459.

(13) Hansson, G. C.; Simons, K.; van Meer, G. Two Strains of the Madin-Darby Canine Kidney (MDCK) Cell Line Have Distinct Glycosphingolipid Compositions. EMBO J. 1986, 5 (3), 483-489. 
(14) Nicolson, G. L. The Fluid-Mosaic Model of Membrane Structure: Still Relevant to Understanding the Structure, Function and Dynamics of Biological Membranes after More than 40 Years. Biochimica et Biophysica Acta (BBA) Biomembranes 2014, 1838 (6), 1451-1466. https://doi.org/10.1016/j.bbamem.2013.10.019.

(15) Bretscher, M. S. Membrane Structure: Some General Principles. Science 1973, 181 (4100), 622-629. https://doi.org/10.1126/science.181.4100.622.

(16) Daleke, D. L. Regulation of Transbilayer Plasma Membrane Phospholipid Asymmetry. Journal of Lipid Research 2003, 44 (2), 233-242. https://doi.org/10.1194/jlr.R200019-JLR200.

(17) Regina Todeschini, A.; Hakomori, S. Functional Role of Glycosphingolipids and Gangliosides in Control of Cell Adhesion, Motility, and Growth, through Glycosynaptic Microdomains. Biochimica et Biophysica Acta (BBA) - General Subjects 2008, $1780 \quad$ (3), 421-433. https://doi.org/10.1016/j.bbagen.2007.10.008.

(18) Engelman, D. M. Membranes Are More Mosaic than Fluid. Nature 2005, 438 (7068), 578-580. https://doi.org/10.1038/nature04394.

(19) Lingwood, D.; Simons, K. Lipid Rafts As a Membrane-Organizing Principle. Science 2010, 327 (5961), 46-50. https://doi.org/10.1126/science.1174621.

(20) Van Meer, G.; Stelzer, E. H.; Wijnaendts-van-Resandt, R. W.; Simons, K. Sorting of Sphingolipids in Epithelial (Madin-Darby Canine Kidney) Cells. J. Cell Biol. 1987, 105 (4), 1623-1635.

(21) Tillack, T. W.; Allietta, M.; Moran, R. E.; Young, W. W. Localization of Globoside and Forssman Glycolipids on Erythrocyte Membranes. Biochimica et Biophysica Acta (BBA) - Biomembranes 1983, 733 (1), 15-24. https://doi.org/10.1016/0005-2736(83)90086-X.

(22) Rock, P.; Allietta, M.; Young, W. W.; Thompson, T. E.; Tillack, T. W. Organization of Glycosphingolipids in Phosphatidylcholine Bilayers: Use of Antibody Molecules and Fab Fragments as Morphologic Markers. Biochemistry 1990, 29 (36), 8484-8490. https://doi.org/10.1021/bi00488a040.

(23) Rock, P.; Allietta, M.; Young, W. W.; Thompson, T. E.; Tillack, T. W. Ganglioside GM1 and Asialo-GM1 at Low Concentration Are Preferentially Incorporated into the Gel Phase in Two-Component, Two-Phase Phosphatidylcholine Bilayers. Biochemistry 1991, 30 (1), 19-25. https://doi.org/10.1021/bi00215a003.

(24) Sorice, M.; Parolini, I.; Sansolini, T.; Garofalo, T.; Dolo, V.; Sargiacomo, M.; Tai, T.; Peschle, C.; Torrisi, M. R.; Pavan, A. Evidence for the Existence of Ganglioside-Enriched Plasma Membrane Domains in Human Peripheral Lymphocytes. J. Lipid Res. 1997, 38 (5), 969-980.

(25) Yamamura, S.; Handa, K.; Hakomori, S. A Close Association of GM3 with C-Src and Rho in GM3-Enriched Microdomains at the B16 Melanoma Cell Surface Membrane: A Preliminary Note. Biochemical and Biophysical Research Communications 1997, 236 (1), 218-222. https://doi.org/10.1006/bbrc.1997.6933.

(26) Okada, Y.; Mugnai, G.; Bremer, E. G.; Hakomori, S. -i. Glycosphingolipids in Detergent-Insoluble Substrate Attachment Matrix (DISAM) Prepared from Substrate Attachment Material (SAM): Their Possible Role in Regulating Cell Adhesion. Experimental Cell Research 1984, 155 (2), 448-456 
(27) Brown, D. A.; London, E. Structure of Detergent-Resistant Membrane Domains: Does Phase Separation Occur in Biological Membranes? Biochem. Biophys. Res. Commun. 1997, 240 (1), 1-7. https://doi.org/10.1006/bbrc.1997.7575.

(28) Simons, K.; Ikonen, E. Functional Rafts in Cell Membranes. Nature 1997, 387 (6633), 569-572.

(29) Fan, J.; Sammalkorpi, M.; Haataja, M. Formation and Regulation of Lipid Microdomains in Cell Membranes: Theory, Modeling, and Speculation. FEBS $\begin{array}{llll}\text { Letters } & \mathbf{2 0 1 0} & 584 & \text { (9), }\end{array}$ https://doi.org/10.1016/j.febslet.2009.10.051.

(30) Simons, K.; Toomre, D. Lipid Rafts and Signal Transduction. Nature Reviews Molecular Cell Biology 2000, 1 (1), 31-39. https://doi.org/10.1038/35036052.

(31) Hakomori, S. -i. The Glycosynapse. Proceedings of the National Academy of Sciences 2002, 99 (1), 225-232. https://doi.org/10.1073/pnas.012540899.

(32) Hakomori, S. Structure, Organization, and Function of Glycosphingolipids in Membrane: Current Opinion in Hematology 2003, 10 (1), 16-24. https://doi.org/10.1097/00062752-200301000-00004.

(33) Iwabuchi, K.; Yamamura, S.; Prinetti, A.; Handa, K.; Hakomori, S. GM3-Enriched Microdomain Involved in Cell Adhesion and Signal Transduction through Carbohydrate-Carbohydrate Interaction in Mouse Melanoma B16 Cells. Journal of Biological Chemistry 1998, 273 (15), 9130-9138. https://doi.org/10.1074/jbc.273.15.9130.

(34) Carquin, M.; D’Auria, L.; Pollet, H.; Bongarzone, E. R.; Tyteca, D. Recent Progress on Lipid Lateral Heterogeneity in Plasma Membranes: From Rafts to Submicrometric Domains. Progress in Lipid Research 2016, 62, 1-24. https://doi.org/10.1016/j.plipres.2015.12.004.

(35) Molecular Biology of the Cell, 5th ed.; Alberts, B., Ed.; Garland Science: New York, 2008.

(36) Van Meer, G.; de Kroon, A. I. P. M. Lipid Map of the Mammalian Cell. Journal of Cell Science 2011, 124 (1), 5-8. https://doi.org/10.1242/jcs.071233.

(37) Thudichum, J. L. W. A Treatise on the Chemical Constitution of the Brain : Based throughout upon Original Researches; London: Bailliere, Tindal and Cox: London, 1884.

(38) Van Meer, G.; Voelker, D. R.; Feigenson, G. W. Membrane Lipids: Where They Are and How They Behave. Nature Reviews Molecular Cell Biology 2008, 9 (2), 112-124. https://doi.org/10.1038/nrm2330.

(39) Mattson, M. P. Membrane Microdomain Signaling Lipid Rafts in Biology and Medicine; Humana Press: Totowa, N.J., 2005.

(40) van Meer, G.; Lisman, Q. Sphingolipid Transport: Rafts and Translocators. Journal of Biological Chemistry 2002, 277 (29), 25855-25858. https://doi.org/10.1074/jbc.R200010200.

(41) Schnaar, R. L.; Kinoshita, T. Glycosphingolipids. In Essentials of Glycobiology; Varki, A., Cummings, R. D., Esko, J. D., Stanley, P., Hart, G. W., Aebi, M., Darvill, A. G., Kinoshita, T., Packer, N. H., Prestegard, J. H., et al., Eds.; Cold Spring Harbor Laboratory Press: Cold Spring Harbor (NY), 2015.

(42) Pascher, I.; Sundell, S. Molecular Arrangements in Sphingolipids. The Crystal Structure of Cerebroside. Chemistry and Physics of Lipids 1977, 20 (3), 175191. https://doi.org/10.1016/0009-3084(77)90033-0. 
(43) Hakomori, S.-I. Tumor-Associated Glycolipid Antigens, Their Metabolism and Organization. Chemistry and Physics of Lipids 1986, 42 (1-3), 209-233. https://doi.org/10.1016/0009-3084(86)90054-X.

(44) Strömberg, N.; Nyholm, P. G.; Pascher, I.; Normark, S. Saccharide Orientation at the Cell Surface Affects Glycolipid Receptor Function. Proceedings of the National Academy of Sciences 1991, 88 (20), 9340-9344. https://doi.org/10.1073/pnas.88.20.9340.

(45) Bucior, I.; Scheuring, S.; Engel, A.; Burger, M. M. Carbohydrate-Carbohydrate Interaction Provides Adhesion Force and Specificity for Cellular Recognition. The Journal of Cell Biology 2004, 165 (4), 529-537. https://doi.org/10.1083/jcb.200309005.

(46) Miljan, E. A.; Bremer, E. G. Regulation of Growth Factor Receptors by Gangliosides. Science Signaling 2002, 2002 (160), re15-re15. https://doi.org/10.1126/stke.2002.160.re15.

(47) Maggio, B.; Fanani, M. L.; Rosetti, C. M.; Wilke, N. Biophysics of Sphingolipids II. Glycosphingolipids: An Assortment of Multiple Structural Information Transducers at the Membrane Surface. Biochimica et Biophysica Acta (BBA) Biomembranes 2006, 1758 (12), 1922-1944. https://doi.org/10.1016/j.bbamem.2006.04.020.

(48) Koldsø, H.; Shorthouse, D.; Hélie, J.; Sansom, M. S. P. Lipid Clustering Correlates with Membrane Curvature as Revealed by Molecular Simulations of Complex Lipid Bilayers. PLoS Computational Biology 2014, 10 (10), e1003911. https://doi.org/10.1371/journal.pcbi.1003911.

(49) Van Meer, G.; Simons, K. The Function of Tight Junctions in Maintaining Differences in Lipid Composition between the Apical and the Basolateral Cell Surface Domains of MDCK Cells. The EMBO Journal 1986, 5 (7), 1455-1464. https://doi.org/10.1002/j.1460-2075.1986.tb04382.x.

(50) Fenderson, B. A. A Multivalent Lacto-N-Fucopentaose III-Lysyllysine Conjugate Decompacts Preimplantation Mouse Embryos, While the Free Oligosaccharide Is Ineffective. Journal of Experimental Medicine 1984, 160 (5), 1591-1596. https://doi.org/10.1084/jem.160.5.1591.

(51) Eggens, I.; Fenderson, B.; Toyokuni, T. T.; Dean, B.; Stroud, M.; Hakomori, S.-I. Specific Interaction between LeX and LeX Determinants. The Journal of Biological Chemistry 1989, 264 (16), 9476-9484.

(52) Kojima, N.; Hakomori, S. -i. Specific Interaction between Gangliotriaosylceramide (Gg3) and Sialosyllactosylceramide (GM3) as a Basis for Specific Cellular Recognition between Lymphoma and Melanoma Cells. The Journal of Biological Chemistry 1989, 264 (34), 20159-20162.

(53) Geyer, A.; Gege, C.; Schmidt, R. R. Calcium-Dependent CarbohydrateCarbohydrate Recognition between LewisX Blood Group Antigens. Angewandte Chemie 2000, 39 (18), 3245-3249. https://doi.org/10.1002/1521-3773(20000915)39:18<3245::AIDANIE3245>3.0.CO;2-9.

(54) Hakomori, S. Carbohydrate-to-Carbohydrate Interaction, through Glycosynapse, as a Basis of Cell Recognition and Membrane Organization. Glycoconjugate Journal 2004, $21 \quad$ (3/4), 125-137. https://doi.org/10.1023/B:GLYC.0000044844.95878.cf. 
(55) Pérez, S.; Mouhous-Riou, N.; Nifant'ev, N. E.; Tsvetkov, Y. E.; Bachet, B.; Imberty, A. Crystal and Molecular Structure of a Histo-Blood Group Antigen Involved in Cell Adhesion: The Lewis x Trisaccharide. Glycobiology 1996, 6 (5), 537-542. https://doi.org/10.1093/glycob/6.5.537.

(56) Bucior, I.; Burger, M. M. Carbohydrate-Carbohydrate Interactions in Cell Recognition. Current Opinion in Structural Biology 2004, 14 (5), 631-637. https://doi.org/10.1016/j.sbi.2004.08.006.

(57) Spillmann, D. Carbohydrates in Cellular Recognition: From Leucine-Zipper to Sugar-Zipper? Glycoconjugate Journal 1994, 11 (3), 169-171. https://doi.org/10.1007/BF00731214.

(58) Spillmann, D.; Burger, M. M. Carbohydrate-Carbohydrate Interactions in Adhesion. Journal of Cellular Biochemistry 1996, 61 (4), 562-568. https://doi.org/10.1002/(SICI)1097-4644(19960616)61:4<562::AIDJCB9>3.0.CO;2-M.

(59) Prokazova, N. V.; Samovilova, N. N.; Gracheva, E. V.; Golovanova, N. K. Ganglioside GM3 and Its Biological Functions. Biochemistry (Moscow) 2009, 74 (3), 235-249. https://doi.org/10.1134/S0006297909030018.

(60) Hakomori, S.-I.; Handa, K. GM3 and Cancer. Glycoconjugate Journal 2015, 32 (1-2), 1-8. https://doi.org/10.1007/s10719-014-9572-4.

(61) Bremer, E. G.; Schlessinger, J.; Hakomori, S.-I. Ganglioside-Mediated Modulation of Cell Growth. Journal of Biological Chemistry 1986, 261 (5), 2434-2440.

(62) Zheng, M.; Fang, H.; Tsuruoka, T.; Tsuji, T.; Sasaki, T.; Hakomori, S. Regulatory Role of GM3 Ganglioside in Alpha 5 Beta 1 Integrin Receptor for FibronectinMediated Adhesion of FUA169 Cells. J. Biol. Chem. 1993, 268 (3), 2217-2222.

(63) Alessandri, G.; de Cristan, G.; Ziche, M.; Cappa, A. P. M.; Gullino, P. M. Growth and Motility of Microvascular Endothelium Are Modulated by the Relative Concentration of Gangliosides in the Medium. Journal of Cellular Physiology 1992, 151 (1), 23-28. https://doi.org/10.1002/jcp.1041510105.

(64) Ziche, M.; Morbidelli, L.; Alessandri, G.; Gullino, P. M. Angiogenesis Can Be Stimulated or Repressed in Vivo by a Change in GM3:GD3 Ganglioside Ratio. Lab. Invest. 1992, 67 (6), 711-715.

(65) Abate, L. E.; Mukherjee, P.; Seyfried, T. N. Gene-Linked Shift in Ganglioside Distribution Influences Growth and Vascularity in a Mouse Astrocytoma: Gangliosides and Angiogenesis. Journal of Neurochemistry 2006, 98 (6), 19731984. https://doi.org/10.1111/j.1471-4159.2006.04097.x.

(66) Kojima, N.; Shiota, M.; Sadahira, Y.; Handa, K.; Hakomori, S. -i. Cell Adhesion in a Dynamic Flow System as Compared to Static System. The Journal of Biological Chemistry 1992, 267 (24), 17264-17270.

(67) Saha, S.; Mohanty, K. C.; Mallick, P. Gangliosides Enhance Migration of Mouse B16-Melanoma Cells through Artificial Basement Membrane Alone or in Presence of Laminin or Fibronectin. Indian Journal of Experimental Biology 2005, 43, 1130-1138.

(68) Zhai, X.; Li, X.-M.; Momsen, M. M.; Brockman, H. L.; Brown, R. E. Lactosylceramide: Lateral Interactions with Cholesterol. Biophysical Journal 2006, 91 (7), 2490-2500. https://doi.org/10.1529/biophysj.106.084921. 
(69) Parkin, E. T.; Turner, A. J.; Hooper, N. M. Differential Effects of Glycosphingolipids on the Detergent-Insolubility of the Glycosylphosphatidylinositol-Anchored Membrane Dipeptidase. Biochemical Journal 2001, 358 (1), 209-216. https://doi.org/10.1042/bj3580209.

(70) Iwabuchi, K.; Prinetti, A.; Sonnino, S.; Mauri, L.; Kobayashi, T.; Ishii, K.; Kaga, N.; Murayama, K.; Kurihara, H.; Nakayama, H.; et al. Involvement of Very Long Fatty Acid-Containing Lactosylceramide in Lactosylceramide-Mediated Superoxide Generation and Migration in Neutrophils. Glycoconjugate Journal 2008, 25 (4), 357-374. https://doi.org/10.1007/s10719-007-9084-6.

(71) Brown, D. A.; London, E. Functions of Lipid Rafts in Biological Membranes. Annual Review of Cell and Developmental Biology 1998, 14 (1), 111-136. https://doi.org/10.1146/annurev.cellbio.14.1.111.

(72) Brown, D. A.; London, E. Structure and Function of Sphingolipid- and Cholesterol-Rich Membrane Rafts. J. Biol. Chem. 2000, 275 (23), 17221-17224. https://doi.org/10.1074/jbc.R000005200.

(73) Symington, F. W.; Hedges, D. L.; Hakomori, S. CDw17: A Neutrophil Glycolipid Antigen Regulated by Activation. The Journal of Immunology 1985, 134 (4), 2498-2506.

(74) Lehrer, R. I. Neutrophils and Host Defense. Annals of Internal Medicine 1988, 109 (2), 127. https://doi.org/10.7326/0003-4819-109-2-127.

(75) Iwabuchi, K.; Nagaoka, I. Lactosylceramide-Enriched Glycosphingolipid Signaling Domain Mediates Superoxide Generation from Human Neutrophils. Blood 2002, 100 (4), 1454-1464.

(76) Iwabuchi, K.; Masuda, H.; Kaga, N.; Nakayama, H.; Matsumoto, R.; Iwahara, C.; Yoshizaki, F.; Tamaki, Y.; Kobayashi, T.; Hayakawa, T.; et al. Properties and Functions of Lactosylceramide from Mouse Neutrophils. Glycobiology 2015, 25 (6), 655-668. https://doi.org/10.1093/glycob/cwv008.

(77) Hahn, P. Y.; Evans, S. E.; Kottom, T. J.; Standing, J. E.; Pagano, R. E.; Limper, A. H. Pneumocystis Carinii Cell Wall $\beta$-Glucan Induces Release of Macrophage Inflammatory Protein-2 from Alveolar Epithelial Cells via a LactosylceramideMediated Mechanism. Journal of Biological Chemistry 2003, 278 (3), 20432050. https://doi.org/10.1074/jbc.M209715200.

(78) Sato, T.; Iwabuchi, K.; Nagaoka, I.; Adachi, Y.; Ohno, N.; Tamura, H.; Seyama, K.; Fukuchi, Y.; Nakayama, H.; Yoshizaki, F.; et al. Induction of Human Neutrophil Chemotaxis by Candida Albicans-Derived -1,6-Long Glycoside Side-ChainBranched -Glucan. Journal of Leukocyte Biology 2006, 80 (1), 204-211. https://doi.org/10.1189/jlb.0106069.

(79) Won, J.-S.; Singh, A. K.; Singh, I. Lactosylceramide: A Lipid Second Messenger in Neuroinflammatory Disease. Journal of Neurochemistry 2007, 103 (s1), 180-191. https://doi.org/10.1111/j.1471-4159.2007.04822.x.

(80) Nakayama, H.; Yoshizaki, F.; Prinetti, A.; Sonnino, S.; Mauri, L.; Takamori, K.; Ogawa, H.; Iwabuchi, K. Lyn-Coupled LacCer-Enriched Lipid Rafts Are Required for CD11b/CD18-Mediated Neutrophil Phagocytosis of Nonopsonized Microorganisms. Journal of Leukocyte Biology 2008, 83 (3), 728-741. https://doi.org/10.1189/jlb.0707478.

(81) Iwabuchi, K.; Nakayama, H.; Masuda, H.; Kina, K.; Ogawa, H.; Takamori, K. Membrane Microdomains in Immunity: Glycosphingolipid-Enriched DomainMediated Innate Immune Responses. BioFactors 2012, 38 (4), 275-283. https://doi.org/10.1002/biof.1017. 
(82) Kapuscinski, J. DAPI: A DNA-Specific Fluorescent Probe. Biotech Histochem 1995, 70 (5), 220-233.

(83) Shapiro, H. M. Flow Cytometric Estimation of DNA and RNA Content in Intact Cells Stained with Hoechst 33342 and Pyronin Y. Cytometry 1981, 2 (3), 143150. https://doi.org/10.1002/cyto.990020302.

(84) Hoechst 33342. In Handbook of Fluorescent Dyes and Probes; John Wiley \& Sons, Inc: Hoboken, NJ, USA, 2015; pp 238-243. https://doi.org/10.1002/9781119007104.ch85.

(85) Dohi, T.; Hakomori, S. -i. An IgG3 Monoclonal Antibody Established after Immunization with GM3 Lactone: Immunochemical Specificity and Inhibition of Melanoma Cell Growth in Vitro and in Vivo. Cancer Research 1988, 48, 5680-5685.

(86) Kojima, N.; Hakomori, S. -i. Cell Adhesion, Spreading, and Motility of GM3Expressing Cells Based on Glycolipid-Glycolipid Interaction. The Journal of Biological Chemistry 1991, 266 (26), 17552-17558.

(87) Kučerka, N.; Nieh, M.-P.; Katsaras, J. Fluid Phase Lipid Areas and Bilayer Thicknesses of Commonly Used Phosphatidylcholines as a Function of Temperature. Biochimica et Biophysica Acta (BBA) - Biomembranes 2011, 1808 (11), 2761-2771. https://doi.org/10.1016/j.bbamem.2011.07.022.

(88) Davis, P. J.; Fleming, B. D.; Coolbear, K. P.; Keough, K. M. W. Gel to LiquidCrystalline Transition Temperatures of Water Dispersions of Two Pairs of Positional Isomers of Unsaturated Mixed-Acid Phosphatidylcholines. $\begin{array}{lllll}\text { Biochemistry } & \text { 1981, } & \end{array}$ https://doi.org/10.1021/bi00515a051.

(89) Phase Transition Temperatures for Glycerophospholipids | Avanti Polar Lipids https://avantilipids.com/tech-support/physical-properties/phasetransition-temps/ (accessed Nov 10, 2018).

(90) 1-Palmitoyl-2-Oleoyl-Sn-Glycero-3-Phosphocholine (Chloroform, 850457C). Sigma-Aldrich, 2018.

(91) Li, X.-M.; Momsen, M. M.; Brockman, H. L.; Brown, R. E. Lactosylceramide: Effect of Acyl Chain Structure on Phase Behavior and Molecular Packing. Biophysical Journal 2002, 83 (3), 1535-1546. https://doi.org/10.1016/S00063495(02)73923-4.

(92) D-Lactosyl-ß-1,1'-N-Palmitoyl-D-Erythro-Sphingosine (860576P). Avanti Polar Lipids, Inc., 2018.

(93) D-Lactosyl-ß-1-1'-D-Erythro-Sphingosine (42137). Sigma-Aldrich, 2018.

(94) Ganglioside GM3 (Bovine Milk, 860058). Avanti Polar Lipids, Inc., 2018.

(95) Fidelio, G. D.; Maggio, B.; Cumar, F. A. Molecular Parameters and Physical State of Neutral Glycosphingolipids and Gangliosides in Monolayers at Different Temperatures. Biochimica et Biophysica Acta (BBA) - Biomembranes 1986, 854 (2), 231-239. https://doi.org/10.1016/0005-2736(86)90115-X.

(96) Koynova, R.; Caffrey, M. Phases and Phase Transitions of the Sphingolipids. Biochimica et Biophysica Acta (BBA) - Lipids and Lipid Metabolism 1995, 1255 (3), 213-236. https://doi.org/10.1016/0005-2760(94)00202-A.

(97) Maggio, B.; Ariga, T.; Sturtevant, J. M.; Yu, R. K. Thermotropic Behavior of Glycosphingolipids in Aqueous Dispersions. Biochemistry 1985, 24 (5), 10841092. https://doi.org/10.1021/bi00326a003. 
(98) Kaiser, R. D.; London, E. Determination of the Depth of BODIPY Probes in Model Membranes by Parallax Analysis of £uorescence Quenching. Biochimica et Biophysica Acta 10.

(99) $\beta$-BODIPYTM 500/510 C12-HPC (D3793). Thermo Fisher Scientific, 2018.

(100) Faller, R. Molecular Modeling of Lipid Probes and Their Influence on the Membrane. Biochimica et Biophysica Acta (BBA) - Biomembranes 2016, 1858 (10), 2353-2361. https://doi.org/10.1016/j.bbamem.2016.02.014.

(101) Skaug, M. J.; Longo, M. L.; Faller, R. The Impact of Texas Red on Lipid Bilayer Properties. The Journal of Physical Chemistry B 2011, 115 (26), 8500-8505. https://doi.org/10.1021/jp203738m.

(102) Skaug, M. J.; Longo, M. L.; Faller, R. Computational Studies of Texas Red-1,2Dihexadecanoyl- $S n$-Glycero-3-Phosphoethanolamine-Model Building and Applications. The Journal of Physical Chemistry B 2009, 113 (25), 8758-8766. https://doi.org/10.1021/jp902877y.

(103) Texas Red ${ }^{\mathrm{TM}}$ DHPE (T1395MP). Thermo Fisher Scientific, 2018.

(104) D-Lactosyl-ß-1,1'-N-Palmitoyl-D-Erythro-Sphingosine (1507). Matreya LLC, 2018.

(105) D-Lactosyl-ß-1-1'-D-Erythro-Sphingosine (2088). Matreya LLC, 2018.

(106) Watson, E. S.; O’Neill, J. Differential Microcalorimeter. US3263484, August 2, 1966.

(107) Watson, E. S.; O’Neill, M. J.; Justin, J.; Brenner, N. A Differential Scanning Calorimeter for Quantitative Differential Thermal Analysis. Analytical Chemistry 1964, 36 (7), 1233-1238. https://doi.org/10.1021/ac60213a019.

(108) Bhadeshia, H. K. D. H. Differential Scanning Calorimetry, 2002.

(109) Schmidt, C.; Rittmeier-Kettner, M.; Becker, H.; Ellert, J.; Krombach, R.; Schneider, G. M. Differential Thermal Analysis (DTA) and Differential Scanning Calorimetry (DSC) at High Pressures. Experimental Techniques and Selected Results. Thermochimica Acta 1994, 238, 321-336. https://doi.org/10.1016/S0040-6031(94)85217-0.

(110) Schwabe, L. Thermoanalyse. In Moderne Pharmazeutische Technologie; Kapitel 3.5; Freie Universität Berlin, 2009.

(111) McConnell, H. M.; Tamm, L. K.; Weis, R. M. Periodic Structures in Lipid Monolayer Phase Transitions. Proceedings of the National Academy of Sciences 1984, 81 (10), 3249-3253. https://doi.org/10.1073/pnas.81.10.3249.

(112) Tamm, L. K.; McConnell, H. M. Supported Phospholipid Bilayers. Biophysical Journal 1985, 47 (1), 105-113. https://doi.org/10.1016/S00063495(85)83882-0.

(113) Charrier, A.; Thibaudau, F. Main Phase Transitions in Supported Lipid SingleBilayer. Biophysical Journal 2005, 89 (2), 1094-1101. https://doi.org/10.1529/biophysj.105.062463.

(114) Brian, A. A.; McConnell, H. M. Allogeneic Stimulation of Cytotoxic T Cells by Supported Planar Membranes. Proceedings of the National Academy of Sciences 1984, 81 (19), 6159-6163. https://doi.org/10.1073/pnas.81.19.6159.

(115) Reimhult, E.; Höök, F.; Kasemo, B. Intact Vesicle Adsorption and Supported Biomembrane Formation from Vesicles in Solution: Influence of Surface Chemistry, Vesicle Size, Temperature, and Osmotic Pressure †. Langmuir 2003, 19 (5), 1681-1691. https://doi.org/10.1021/la0263920.

(116) Kalb, E.; Frey, S.; Tamm, L. K. Formation of Supported Planar Bilayers by Fusion of Vesicles to Supported Phospholipid Monolayers. Biochimica et Biophysica 


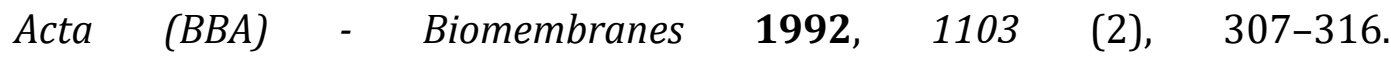
https://doi.org/10.1016/0005-2736(92)90101-Q.

(117) Csúcs, G.; Ramsden, J. J. Interaction of Phospholipid Vesicles with Smooth Metal-Oxide Surfaces. Biochimica et Biophysica Acta (BBA) - Biomembranes 1998, 1369 (1), 61-70. https://doi.org/10.1016/S0005-2736(97)00209-5.

(118) Richter, R. P.; Brisson, A. R. Following the Formation of Supported Lipid Bilayers on Mica: A Study Combining AFM, QCM-D, and Ellipsometry. $\begin{array}{llll}\text { Biophysical Journal 2005, } 88 \quad \text { (5), } & \text { 3422-3433. }\end{array}$ https://doi.org/10.1529/biophysj.104.053728.

(119) Sackmann, E. Supported Membranes: Scientific and Practical Applications. Science 1996, 271 (5245), 43-48. https://doi.org/10.1126/science.271.5245.43.

(120) Ulrich, A. S. Biophysical Aspects of Using Liposomes as Delivery Vehicles. 22.

(121) Kiessling, V.; Tamm, L. K. Measuring Distances in Supported Bilayers by Fluorescence Interference-Contrast Microscopy: Polymer Supports and SNARE Proteins. Biophysical Journal 2003, 84 (1), 408-418. https://doi.org/10.1016/S0006-3495(03)74861-9.

(122) Johnston, L. J. Nanoscale Imaging of Domains in Supported Lipid Membranes. Langmuir 2007, 23 (11), 5886-5895. https://doi.org/10.1021/la070108t.

(123) Eeman, M.; Deleu, M. From Biological Membranes to Biomimetic Model Membranes. Biotechnol. Agron. Soc. Environ. 2010, 18.

(124) Schütte, O. M. Structure and Dynamics of Artificial Lipid Membranes Containing the Glycosphingolipid Gb3, Georg-August-Universität: Göttingen, 2015.

(125) Luckey, M. Membrane Structural Biology: With Biochemical and Biophysical Foundations; Cambridge University Press: Cambridge ; New York, 2008.

(126) Winter, R. Struktur und Dynamik von Modell-Biomembranen. Chemie in $\begin{array}{lllll}\text { unserer Zeit } & \text { 1990, } & \text { 71-81. }\end{array}$ https://doi.org/10.1002/ciuz.19900240207.

(127) Binnig, G.; Rohrer, H. Scanning Tunneling Microscopy. IBM Journal of Research and Development 1983, 30 (4), 355-369.

(128) Binnig, G.; Rohrer, H.; Gerber, C.; Weibel, E. Surface Studies by Scanning Tunneling Microscopy. Physical Review Letters 1982, 49 (1), 57-61. https://doi.org/10.1103/PhysRevLett.49.57.

(129) Silbernagl, D. Bestimmung mechanischer Eigenschaften dünner Polymerfilme mittels AFM-Kraft-Abstands-Kurven, Fachbereich Chemie, Pharmazie und Geowissenschaften, Johannes Gutenberg-Universität: Mainz, 2009.

(130) Binnig, G.; Quate, C. F.; Gerber, C. Atomic Force Microscope. Physical Review Letters 1986, 56 (9), 930-933. https://doi.org/10.1103/PhysRevLett.56.930.

(131) Ando, T.; Uchihashi, T.; Scheuring, S. Filming Biomolecular Processes by HighSpeed Atomic Force Microscopy. Chemical Reviews 2014, 114 (6), 3120-3188. https://doi.org/10.1021/cr4003837.

(132) Butt, H.-J.; Cappella, B.; Kappl, M. Force Measurements with the Atomic Force Microscope: Technique, Interpretation and Applications. Surface Science Reports 2005, $59 \quad$ (1-6), https://doi.org/10.1016/j.surfrep.2005.08.003.

(133) Cappella, B.; Dietler, G. Force-Distance Curves by Atomic Force Microscopy. Surface Science Reports 1999, 34 (1-3), 1-104. https://doi.org/10.1016/S0167-5729(99)00003-5. 
(134) Kramer, C. Verknüpfung Zwischen Plasmamembran Und Zytoskelett: Charakterisierung Der Organisation von Ezrin Und F-Aktin an Artifiziellen Lipidmembranen, Georg-August-Universität Göttingen: Göttingen, 2016.

(135) Kamprad, N.; Witt, H.; Schröder, M.; Kreis, C. T.; Bäumchen, O.; Janshoff, A.; Tarantola, M. Adhesion Strategies of Dictyostelium Discoideum - a Force Spectroscopy Study. Nanoscale 2018, 10 (47), 22504-22519. https://doi.org/10.1039/C8NR07107A.

(136) Sariisik, E.; Popov, C.; Müller, J. P.; Docheva, D.; Clausen-Schaumann, H.; Benoit, M. Decoding Cytoskeleton-Anchored and Non-Anchored Receptors from Single-Cell Adhesion Force Data. Biophysical Journal 2015, 109 (7), 13301333. https://doi.org/10.1016/j.bpj.2015.07.048.

(137) Hutter, J. L.; Bechhoefer, J. Calibration of Atomic-force Microscope Tips. Review of Scientific Instruments 1993, 64 (7), 1868-1873. https://doi.org/10.1063/1.1143970.

(138) Evans, E.; Ritchie, K.; Merkel, R. Sensitive Force Technique to Probe Molecular Adhesion and Structural Linkages at Biological Interfaces. Biophysical Journal 1995, 68 (6), 2580-2587. https://doi.org/10.1016/S0006-3495(95)80441-8.

(139) Litvinov, R. I.; Shuman, H.; Bennett, J. S.; Weisel, J. W. Binding Strength and Activation State of Single Fibrinogen-Integrin Pairs on Living Cells. Proceedings of the National Academy of Sciences 2002, 99 (11), 7426-7431. https://doi.org/10.1073/pnas.112194999.

(140) Dzementsei, A.; Schneider, D.; Janshoff, A.; Pieler, T. Migratory and Adhesive Properties of Xenopus Laevis Primordial Germ Cells in Vitro. Biology Open 2013, 2 (12), 1279-1287. https://doi.org/10.1242/bio.20135140.

(141) Lorenz, B.; Keller, R.; Sunnick, E.; Geil, B.; Janshoff, A. Colloidal Probe Microscopy of Membrane-Membrane Interactions: From Ligand-Receptor Recognition to Fusion Events. Biophysical Chemistry 2010, 150 (1-3), 54-63. https://doi.org/10.1016/j.bpc.2010.02.008.

(142) Lorenz, B. A Force Spectroscopy Setup to Mimic Cellular Interaction Processes, Georg-August-Universität Göttingen: Göttingen, 2012.

(143) Nečas, D.; Klapetek, P. Gwyddion: An Open-Source Software for SPM Data Analysis. Open Physics 2012, 10 (1). https://doi.org/10.2478/s11534-0110096-2.

(144) Orth, A. Einfluss des Zellkortex auf die Plasmamembran: Modulation von Mikrodomänen in Modellmembranen, Georg-August-Universität Göttingen: Göttingen, 2012.

(145) Atkins, P. W.; de Paula, J. Kurzlehrbuch Physikalische Chemie; Wiley-VCH: Weinheim, 2006.

(146) Lakowicz, J. R. Principles of Fluorescence Spectroscopy, 3rd ed.; Springer: New York, 2006.

(147) von Bünau, G.; Wolff, T. Photochemie, Grundlagen, Methoden, Anwendungen; VCH Verlagsgesellschaft: Weinheim, 1987.

(148) Abbe, E. Beiträge Zur Theorie Des Mikroskops Und Der Mikroskopischen Wahrnehmung. Archiv für mikroskopische Anatomie 1873, 9 (1), 413-418.

(149) Schindelin, J.; Arganda-Carreras, I.; Frise, E.; Kaynig, V.; Longair, M.; Pietzsch, T.; Preibisch, S.; Rueden, C.; Saalfeld, S.; Schmid, B.; et al. Fiji: An Open-Source Platform for Biological-Image Analysis. Nature Methods 2012, 9 (7), 676-682. https://doi.org/10.1038/nmeth.2019. 
(150) Schermelleh, L.; Heintzmann, R.; Leonhardt, H. A Guide to Super-Resolution Fluorescence Microscopy. The Journal of Cell Biology 2010, 190 (2), 165-175. https://doi.org/10.1083/jcb.201002018.

(151) Minsky, M. Memoir on Inventing the Confocal Scanning Microscope. Scanning 1988, 10 (4), 128-138. https://doi.org/10.1002/sca.4950100403.

(152) Axelrod, D.; Koppel, D. E.; Schlessinger, J.; Elson, E.; Webb, W. W. Mobility Measurement by Analysis of Fluorescence Photobleaching Recovery Kinetics. Biophysical Journal 1976, $16 \quad$ (9), 1055-1069. https://doi.org/10.1016/S0006-3495(76)85755-4.

(153) Hoffmann, J. Charakterisierung Der Diffusion Und Der Reaktionskinetik von Peripheren Membranproteinen in Lebenden Zellen, Graduiertenschule für Mathematik und Naturwissenschaften der Universität Bayreuth: Bayreuth, 2015.

(154) Magde, D.; Elson, E. L.; Webb, W. W. Fluorescence Correlation Spectroscopy. II. An Experimental Realization. Biopolymers 1974, 13 (1), 29-61. https://doi.org/10.1002/bip.1974.360130103.

(155) Ambrose, E. J. A Surface Contact Microscope for the Study of Cell Movements. Nature 1956, 178, 1194.

(156) Mattheyses, A. L.; Simon, S. M.; Rappoport, J. Z. Imaging with Total Internal Reflection Fluorescence Microscopy for the Cell Biologist. Journal of Cell Science 2010, 123 (21), 3621-3628. https://doi.org/10.1242/jcs.056218.

(157) Chan, L. L.-Y.; Lai, N.; Shen, D.; Wilkinson, A. R.; Patton, W.; Chan, E.; Kuksin, D.; Lin, B.; Qiu, J. A Rapid Method for Detecting Autophagy Activity in Live Cells Using Cellometer Image Cytometry. In Autophagy: Cancer, Other Pathologies, Inflammation, Immunity, Infection, and Aging; Elsevier, 2016; pp 169-183. https://doi.org/10.1016/B978-0-12-805421-5.00008-2.

(158) Limozin, L.; Sengupta, K. Quantitative Reflection Interference Contrast Microscopy (RICM) in Soft Matter and Cell Adhesion. ChemPhysChem 2009, 10 (16), 2752-2768. https://doi.org/10.1002/cphc.200900601.

(159) Matsuzaki, T.; Ito, K.; Masuda, K.; Kakinuma, E.; Sakamoto, R.; Iketaki, K.; Yamamoto, H.; Suganuma, M.; Kobayashi, N.; Nakabayashi, S.; et al. Quantitative Evaluation of Cancer Cell Adhesion to Self-Assembled MonolayerPatterned Substrates by Reflection Interference Contrast Microscopy. The Journal of Physical Chemistry $B$ 2016, 120 (7), 1221-1227. https://doi.org/10.1021/acs.jpcb.5b11870.

(160) Abercrombie, M.; Ambrose, E. J. Interference Microscope Studies of Cell Contacts in Tissure Culture. Experimental Cell Research 1958, 15, 332-345.

(161) Curtis, A. S. G. The Mechanism of Adhesion of Cells to Glass: A Study by Interference Reflection Microscopy. The Journal of Cell Biology 1964, 20 (2), 199-215. https://doi.org/10.1083/jcb.20.2.199.

(162) Ploem, J. Reflection Contrast Microscopy as a Tool for Investigation of the Attachment of Living Cells to Glass Surface. Blackwell Scientific Publications 1975, 404-421.

(163) Verschueren, H. Interference Reflection Microscopy in Cell Biology: Methodology and Applications. Journal of Cell Science 1985, 75, 279-301.

(164) Gingell, D.; Todd, I. Interference Reflection Microscopy. A Quantitative Theory for Image Interpretation and Its Application to Cell-Substratum Separation Measurement. Biophysical Journal 1979, 26 (3), 507-526. https://doi.org/10.1016/S0006-3495(79)85268-6. 
(165) Izzard, C. S.; Lochner, L. R. Cell-to-Substrate Contacts in Living Fibroblasts: An Interference Reflexion Study with an Evaluation of the Technique. J. Cell Sci. 1976, 21, 129-159.

(166) Tumor Models in Cancer Research; Teicher, B. A., Ed.; Humana Press: Totowa, NJ, 2011. https://doi.org/10.1007/978-1-60761-968-0.

(167) Overwijk, W. W.; Restifo, N. P. B16 as a Mouse Model for Human Melanoma. Current Protocols in Immunology 2001, 39 (1), 20.1.1-20.1.29. https://doi.org/10.1002/0471142735.im2001s39.

(168) Gennuso, F.; Fernetti, C.; Tirolo, C.; Testa, N.; L’Episcopo, F.; Caniglia, S.; Morale, M. C.; Ostrow, J. D.; Pascolo, L.; Tiribelli, C.; et al. Bilirubin Protects Astrocytes from Its Own Toxicity by Inducing Up-Regulation and Translocation of Multidrug Resistance-Associated Protein 1 (Mrp1). Proceedings of the National Academy of Sciences 2004, $101 \quad$ (8), 2470-2475. https://doi.org/10.1073/pnas.0308452100.

(169) Rajagopal, A.; Pant, A. C.; Simon, S. M.; Chen, Y. In Vivo Analysis of Human Multidrug Resistance Protein 1 (MRP1) Activity Using Transient Expression of Fluorescently Tagged MRP1. Cancer Res. 2002, 62 (2), 391-396.

(170) Hipfner, D. R.; Gauldie, S. D.; Deeley, R. G.; Cole, S. P. Detection of the M(r) 190,000 Multidrug Resistance Protein, MRP, with Monoclonal Antibodies. Cancer Res. 1994, 54 (22), 5788-5792.

(171) Axelrod, D. Total Internal Reflection Fluorescence Microscopy in Cell Biology. Methods in Enzymology 2003, 361, 1-33.

(172) Kaksonen, M.; Toret, C. P.; Drubin, D. G. A Modular Design for the Clathrin- and Actin-Mediated Endocytosis Machinery. Cell 2005, 123 (2), 305-320. https://doi.org/10.1016/j.cell.2005.09.024.

(173) Merrifield, C. J.; Feldman, M. E.; Wan, L.; Almers, W. Imaging Actin and Dynamin Recruitment during Invagination of Single Clathrin-Coated Pits. Nature Cell Biology 2002, 4, 691.

(174) Krylyshkina, O.; Anderson, K. I.; Kaverina, I.; Upmann, I.; Manstein, D. J.; Small, J. V.; Toomre, D. K. Nanometer Targeting of Microtubules to Focal Adhesions. The Journal of Cell Biology 2003, 161 (5), 853-859. https://doi.org/10.1083/jcb.200301102.

(175) Stolarska, M. A.; Rammohan, A. R. Center or Periphery? Modeling the Effects of Focal Adhesion Placement during Cell Spreading. PLOS ONE 2017, 12 (2), e0171430. https://doi.org/10.1371/journal.pone.0171430.

(176) Sawada, M.; Moriya, S.; Shineha, R.; Satomi, S.; Miyagi, T. Comparative Study of Sialidase Activity and GM3 Content in B16 Melanoma Variants with Different Metastatic Potential. Acta Biochimica Polonica 1998, 45 (2), 343-349.

(177) Brillinger, D. R. Data Analysis, Exploratory. International Encyclopedia of Political Science 2011, 531-538.

(178) Humphries, M. J.; Obara, M.; Olden, K.; Yamada, K. M. Role of Fibronectin in Adhesion, Migration, and Metastasis. Cancer Investigation 1989, 7 (4), 373393. https://doi.org/10.3109/07357908909039866.

(179) Mezzenga, R.; Mitsi, M. The Molecular Dance of Fibronectin: Conformational Flexibility Leads to Functional Versatility. Biomacromolecules 2018. https://doi.org/10.1021/acs.biomac.8b01258.

(180) Chen, C. S. Geometric Control of Cell Life and Death. Science 1997, 276 (5317), 1425-1428. https://doi.org/10.1126/science.276.5317.1425. 
(181) Truskey, G. A.; Burmeister, J. S.; Grapa, E.; Reichert, W. M. Total Internal Reflection Fluorescence Microscopy (TIRFM). II. Topographical Mapping of Relative Cell/Substratum Separation Distances. J. Cell. Sci. 1992, 103 ( Pt 2), 491-499.

(182) Adams, J. C. Characterization of Cell-Matrix Adhesion Requirements for the Formation of Fascin Microspikes. Molecular Biology of the Cell 1997, 8 (11), 2345-2363. https://doi.org/10.1091/mbc.8.11.2345.

(183) Adams, J. C. Cell-Matrix Contact Structures. Cellular and Molecular Life Sciences 2001, 58 (3), 371-392. https://doi.org/10.1007/PL00000864.

(184) D’Angelo, G.; Capasso, S.; Sticco, L.; Russo, D. Glycosphingolipids: Synthesis and Functions. FEBS Journal 2013, 280 (24), 6338-6353. https://doi.org/10.1111/febs.12559.

(185) Mao, Y.; Schwarzbauer, J. E. Fibronectin Fibrillogenesis, a Cell-Mediated Matrix Assembly Process. Matrix Biology 2005, 24 (6), 389-399. https://doi.org/10.1016/j.matbio.2005.06.008.

(186) Sun, Z.; Martinez-Lemus, L. A.; Trache, A.; Trzeciakowski, J. P.; Davis, G. E.; Pohl, U.; Meininger, G. A. Mechanical Properties of the Interaction between Fibronectin and A5 $\beta 1$-Integrin on Vascular Smooth Muscle Cells Studied Using Atomic Force Microscopy. American Journal of Physiology-Heart and Circulatory Physiology 2005, 289 (6), H2526-H2535. https://doi.org/10.1152/ajpheart.00658.2004.

(187) Hill, R. P.; Chambers, A. F.; Ling, V.; Harris, J. F. Dynamic Heterogeneity: Rapid Generation of Metastatic Variants in Mouse B16 Melanoma Cells. Science 1984, 224, 998-1001. https://doi.org/10.1126/science.6719130.

(188) Janshoff, A.; Steinem, C. Transport across Artificial Membranes-an Analytical Perspective. Analytical and Bioanalytical Chemistry 2006, 385 (3), 433-451. https://doi.org/10.1007/s00216-006-0305-9.

(189) Kiessling, V.; Wan, C.; Tamm, L. K. Domain Coupling in Asymmetric Lipid Bilayers. Biochimica et Biophysica Acta (BBA) - Biomembranes 2009, 1788 (1), 64-71. https://doi.org/10.1016/j.bbamem.2008.09.003.

(190) Steinem, C.; Janshoff, A. Multicomponent Membranes on Solid Substrates: Interfaces for Protein Binding. Current Opinion in Colloid \& Interface Science 2010, 15 (6), 479-488. https://doi.org/10.1016/j.cocis.2010.06.004.

(191) Seantier, B.; Kasemo, B. Influence of Mono- And Divalent Ions on the Formation of Supported Phospholipid Bilayers via Vesicle Adsorption. Langmuir 2009, 25 (10), 5767-5772. https://doi.org/10.1021/la804172f.

(192) Dimitrievski, K.; Reimhult, E.; Kasemo, B.; Zhdanov, V. P. Simulations of Temperature Dependence of the Formation of a Supported Lipid Bilayer via Vesicle Adsorption. Colloids and Surfaces B: Biointerfaces 2004, 39 (1-2), 7786. https://doi.org/10.1016/j.colsurfb.2004.09.003.

(193) Ohki, S.; Ohshima, H. Interaction and Aggregation of Lipid Vesicles (DLVO Theory versus Modified DLVO Theory). Colloids and Surfaces B: Biointerfaces 1999, 14 (1-4), 27-45. https://doi.org/10.1016/S0927-7765(99)00022-3.

(194) Richter, R.; Mukhopadhyay, A.; Brisson, A. Pathways of Lipid Vesicle Deposition on Solid Surfaces: A Combined QCM-D and AFM Study. Biophysical Journal 2003, 85 (5), 3035-3047. https://doi.org/10.1016/S00063495(03)74722-5. 
(195) Cremer, P. S.; Boxer, S. G. Formation and Spreading of Lipid Bilayers on Planar Glass Supports. The Journal of Physical Chemistry B 1999, 103 (13), 25542559. https://doi.org/10.1021/jp983996x.

(196) Hakomori, S. Carbohydrate-Carbohydrate Interaction as an Initial Step in Cell Recognition. Pure and Applied Chemistry 1991, 63 (4), 473-482. https://doi.org/10.1351/pac199163040473.

(197) Rojo, J.; Morales, J. C.; Penadés, S. Carbohydrate-Carbohydrate Interactions in Biological and Model Systems. In Host-Guest Chemistry; Penadés, S., Ed.; de Meijere, A., Kessler, H., Ley, S. V., Thiem, J., Vögtle, F., Houk, K. N., Lehn, J.-M., Schreiber, S. L., Trost, B. M., Yamamoto, H., Series Eds.; Springer Berlin Heidelberg: Berlin, Heidelberg, 2002; Vol. 218, pp 45-92. https://doi.org/10.1007/3-540-45010-6_2.

(198) Jander, G.; Blasius, E.; Strähle, J.; Schweda, E.; Rossi, R. Lehrbuch der analytischen und präparativen anorganischen Chemie, 16., überarb. Aufl.; Hirzel: Stuttgart, 2006.

(199) Wang, J.; Segatori, L.; Biswal, S. L. Probing the Association of Triblock Copolymers with Supported Lipid Membranes Using Microcantilevers. Soft Matter 2014, 10 (34), 6417-6424. https://doi.org/10.1039/C4SM00928B.

(200) Gräb, O. Solid Supported Model Membranes Containing Plant Glycolipids: A Tool to Study Interactions between Diatom Biomolecules and the Silicalemma in Vitro, Georg-August-Universität: Göttingen, 2017.

(201) Maunula, S.; Björkqvist, Y. J. E.; Slotte, J. P.; Ramstedt, B. Differences in the Domain Forming Properties of N-Palmitoylated Neutral Glycosphingolipids in Bilayer Membranes. Biochimica et Biophysica Acta (BBA) - Biomembranes 2007, 1768 (2), 336-345. https://doi.org/10.1016/j.bbamem.2006.09.003.

(202) Saxena, K.; Zimmermann, P.; Schmidt, R. R.; Shipley, G. G. Bilayer Properties of Totally Synthetic C16:0-Lactosyl-Ceramide. Biophysical Journal 2000, 78 (1), 306-312. https://doi.org/10.1016/S0006-3495(00)76593-3.

(203) Shi, J.; Yang, T.; Kataoka, S.; Zhang, Y.; Diaz, A. J.; Cremer, P. S. GM1 Clustering Inhibits Cholera Toxin Binding in Supported Phospholipid Membranes. Journal of the American Chemical Society 2007, 129 (18), 5954-5961. https://doi.org/10.1021/ja069375w.

(204) McDaniel, R. V.; McIntosh, T. J. X-Ray Diffraction Studies of the Cholera Toxin Receptor, GM1. Biophysical Journal 1986, 49 (1), 94-96. https://doi.org/10.1016/S0006-3495(86)83606-2.

(205) McIntosh, T. J.; Simon, S. A. Long- and Short-Range Interactions between Phospholipid/Ganglioside GM1 Bilayers. Biochemistry 1994, 33 (34), 1047710486. https://doi.org/10.1021/bi00200a032.

(206) Gupta, G.; Surolia, A. Glycosphingolipids in Microdomain Formation and Their Spatial Organization. FEBS Letters 2010, 584 (9), 1634-1641. https://doi.org/10.1016/j.febslet.2009.11.070.

(207) Hakomori, S.; Handa, K. Glycosphingolipid-Dependent Cross-Talk between Glycosynapses Interfacing Tumor Cells with Their Host Cells: Essential Basis to Define Tumor Malignancy. FEBS Letters 2002, 531 (1), 88-92. https://doi.org/10.1016/S0014-5793(02)03479-8.

(208) Witt, H.; Savić, F.; Oelkers, M.; Awan, S. I.; Werz, D. B.; Geil, B.; Janshoff, A. Size, Kinetics, and Free Energy of Clusters Formed by Ultraweak CarbohydrateCarbohydrate Bonds. Biophysical Journal 2016, 110 (7), 1582-1592. https://doi.org/10.1016/j.bpj.2016.03.006. 
(209) Kappl, M.; Butt, H.-J. The Colloidal Probe Technique and Its Application to Adhesion Force Measurements. Particle \& Particle Systems Characterization 2002, $19 \quad$ (3), $129 . \quad$ https://doi.org/10.1002/15214117(200207)19:3<129::AID-PPSC129>3.0.CO;2-G.

(210) Lorenz, B.; Álvarez de Cienfuegos, L.; Oelkers, M.; Kriemen, E.; Brand, C.; Stephan, M.; Sunnick, E.; Yüksel, D.; Kalsani, V.; Kumar, K.; et al. Model System for Cell Adhesion Mediated by Weak Carbohydrate-Carbohydrate Interactions. Journal of the American Chemical Society 2012, 134 (7), 33263329. https://doi.org/10.1021/ja210304j.

(211) Handa, K.; Hakomori, S. Carbohydrate to Carbohydrate Interaction in Development Process and Cancer Progression. Glycoconjugate Journal 2012, 29 (8-9), 627-637. https://doi.org/10.1007/s10719-012-9380-7.

(212) Hinterdorfer, P.; Baumgartner, W.; Gruber, H. J.; Schilcher, K.; Schindler, H. Detection and Localization of Individual Antibody-Antigen Recognition Events by Atomic Force Microscopy. Proc. Natl. Acad. Sci. U.S.A. 1996, 93 (8), 34773481.

(213) Fritz, J.; Katopodis, A. G.; Kolbinger, F.; Anselmetti, D. Force-Mediated Kinetics of Single P-Selectin/Ligand Complexes Observed by Atomic Force Microscopy. Proc. Natl. Acad. Sci. U.S.A. 1998, 95 (21), 12283-12288.

(214) Hanley, W.; McCarty, O.; Jadhav, S.; Tseng, Y.; Wirtz, D.; Konstantopoulos, K. Single Molecule Characterization of P-Selectin/Ligand Binding. Journal of Biological Chemistry 2003, 278 (12), 10556-10561. https://doi.org/10.1074/jbc.M213233200.

(215) Haseley, S. R.; Vermeer, H. J.; Kamerling, J. P.; Vliegenthart, J. F. Carbohydrate Self-Recognition Mediates Marine Sponge Cellular Adhesion. Proc. Natl. Acad. $\begin{array}{llll}\text { Sci. U.S.A. 2001, } 98 \text { (16), } & 9419-9424 .\end{array}$ https://doi.org/10.1073/pnas.151111298.

(216) Hakomori, S. -i. Cell Density-Dependent Changes of Glycolipid Concentrations in Fibroblasts, and Loss of This Response in Virus-Transformed Cells. Proceedings of the National Academy of Sciences 1970, 67 (4), 1741-1747. https://doi.org/10.1073/pnas.67.4.1741.

(217) Hakomori, S. Glycosylation Defining Cancer Malignancy: New Wine in an Old Bottle. Proceedings of the National Academy of Sciences 2002, 99 (16), 1023110233. https://doi.org/10.1073/pnas.172380699.

(218) Usuki, S.; Lyu, S. C.; Sweeley, C. C. Sialidase Activities of Cultured Human Fibroblasts and the Metabolism of GM3 Ganglioside. J. Biol. Chem. 1988, 263 (14), 6847-6853. 



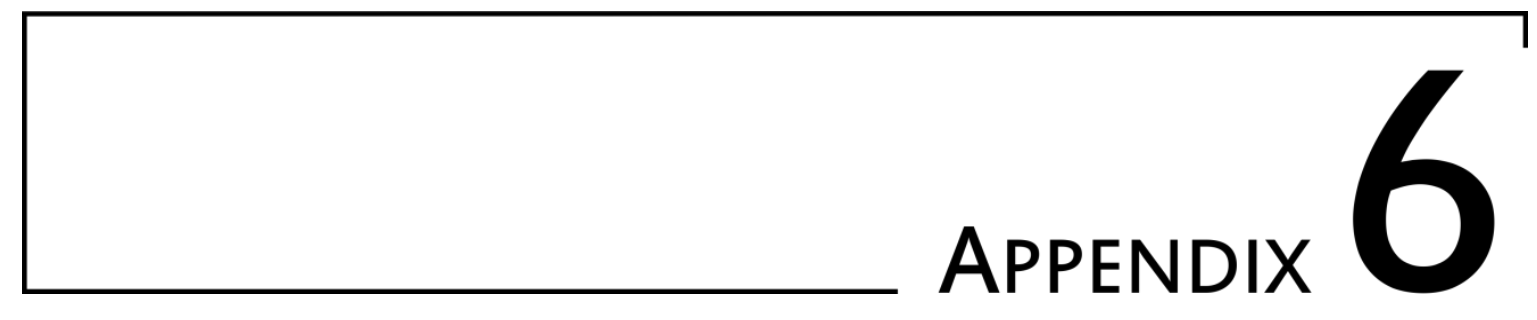

\subsection{TIME SERIES OF FRAP STUDY}

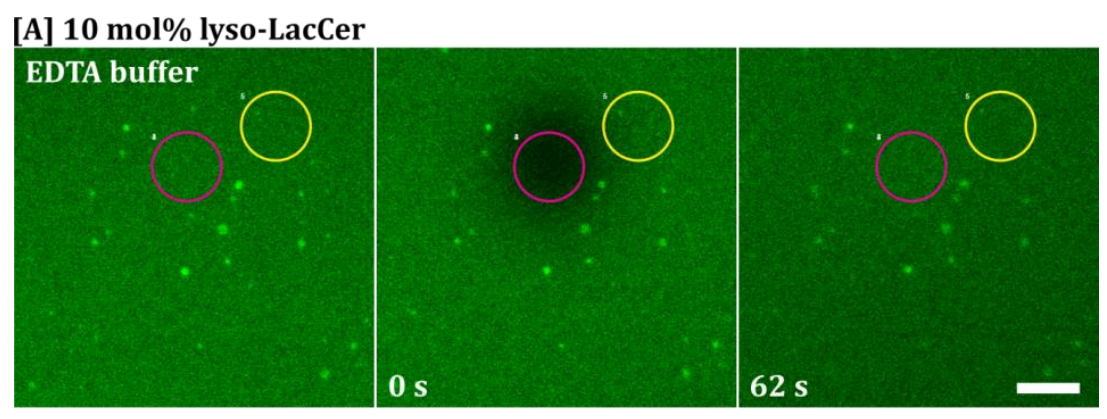

\section{[B] 2 mol\% lyso-LacCer}
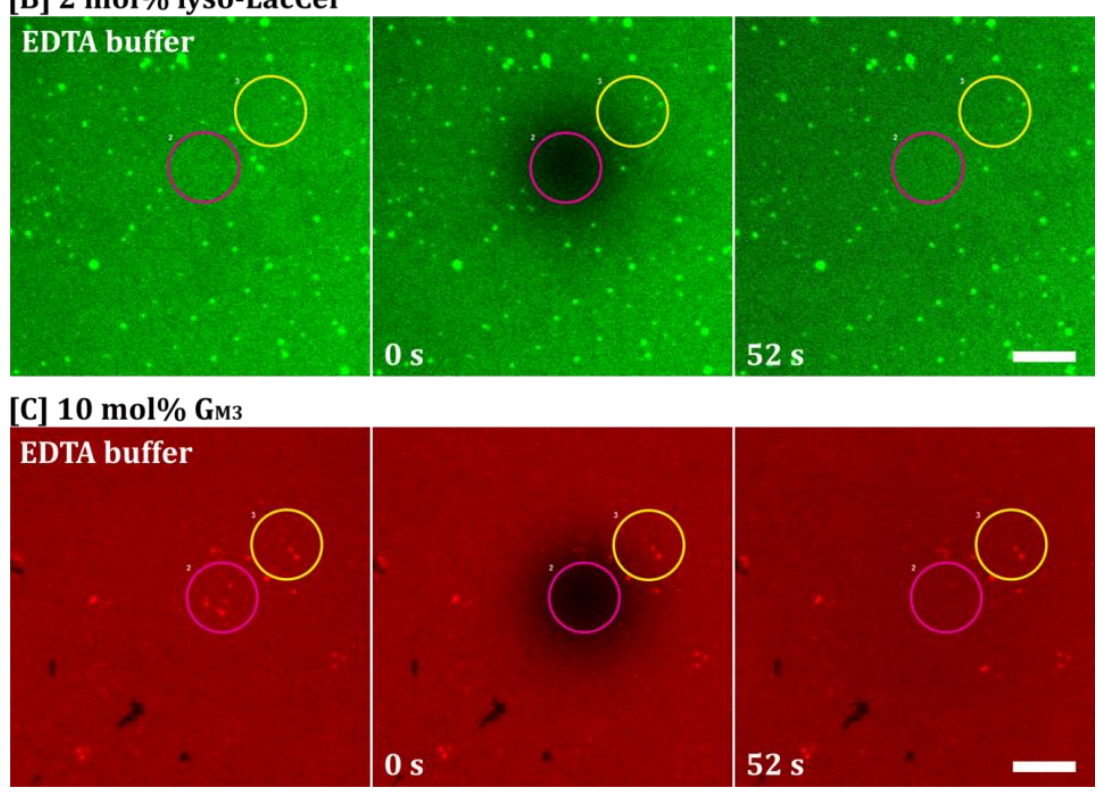

Figure 6.1: Images showing time series of a fluorescence recovery after photobleaching (FRAP) experiment on solid supported lipid membranes consisting of [A] POPC/lyso-LacCer/ $\beta$-Bodipy ${ }^{\mathrm{TM}}$ (89:10:1 mol\%), [B] POPC/lysoLacCer/ $\beta$-BodipyTM $(97: 2: 1 \mathrm{~mol} \%)$ and $[\mathrm{C}]$ POPC/G $/ \mathrm{G}_{\mathrm{M}} / \mathrm{Texas} \operatorname{Red}^{\mathrm{TM}}$ DHPE $(89: 10: 1 \mathrm{~mol} \%)$ on silicon dioxide substrates. The quality of the membranes was analyzed under the absence of a calcium-containing buffer (EDTA). The fluorescent molecules in a desired region of interest a certain range (ROI, yellow) were irreversibly bleached at time $t=0$. Due to the diffusion of surrounding intact fluorophores, the fluorescence intensity was recovered within the ROI. [D] Exemplary FRAP curve of the membrane shown in [A]. Here, the observed fluorescence intensities from the specific ROI were normalized to the fluorescence intensity at the beginning and plotted against the time. Scale bars: $5 \mu \mathrm{m}$. 


\subsection{TOPOGRAPHICAL MAPS (AFM, TAPPING MODE)}

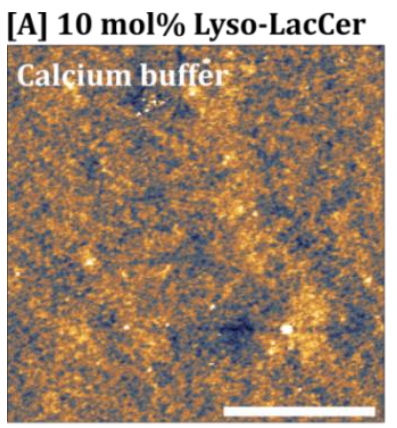

[C] 2 mol\% Lyso-LacCer

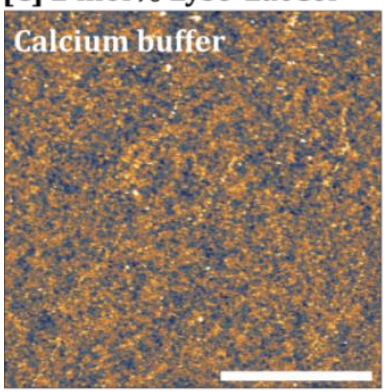

[E] $10 \mathrm{~mol} \%$ Lyso-LacCer

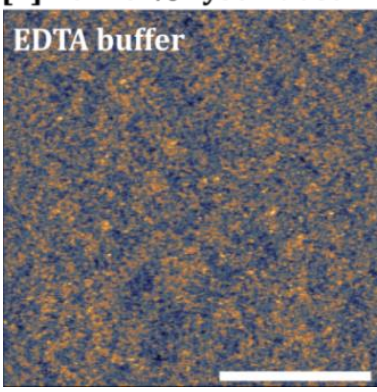

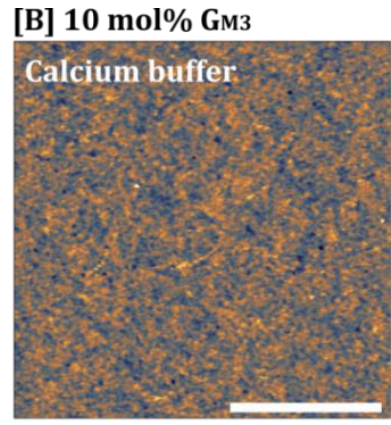

[D] POPC

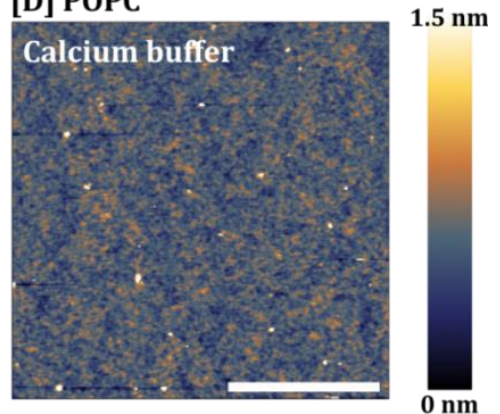

[F] $10 \mathrm{~mol} \%$ GM3

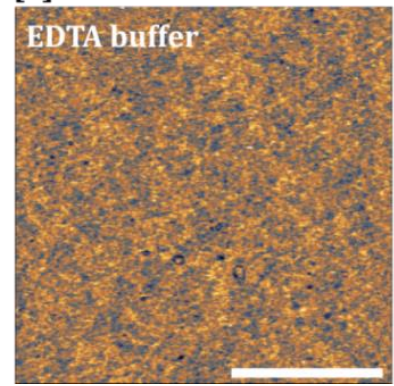

Figure 6.2: Topographic maps (AFM, tapping mode) of lipid membranes consisting of $[\mathrm{A}]+[\mathrm{E}] \mathrm{POPC} / \mathrm{lyso}-$ LacCer/ $\beta$-Bodipy $\quad(89: 10: 1 \mathrm{~mol} \%), \quad[\mathrm{B}] \quad$ POPC/lyso-LacCer/ $\beta$-Bodipy ${ }^{\mathrm{TM}} \quad(97: 2: 1 \mathrm{~mol} \%)$ and $[\mathrm{C}]+[\mathrm{F}]$ POPC/G $/ \mathrm{G}_{\mathrm{M} 3} /$ Texas RedTM DHPE (89:10:1 mol\%) on silicon dioxide substrates. The topography of the membranes was analyzed in presence (calcium buffer) and absence (EDTA buffer) of calcium ions. Scale bar: $2 \mu \mathrm{m}$. 


\subsection{DISTRIBUTION OF MAXIMUM ADHESION FORCES OF B16-F1}
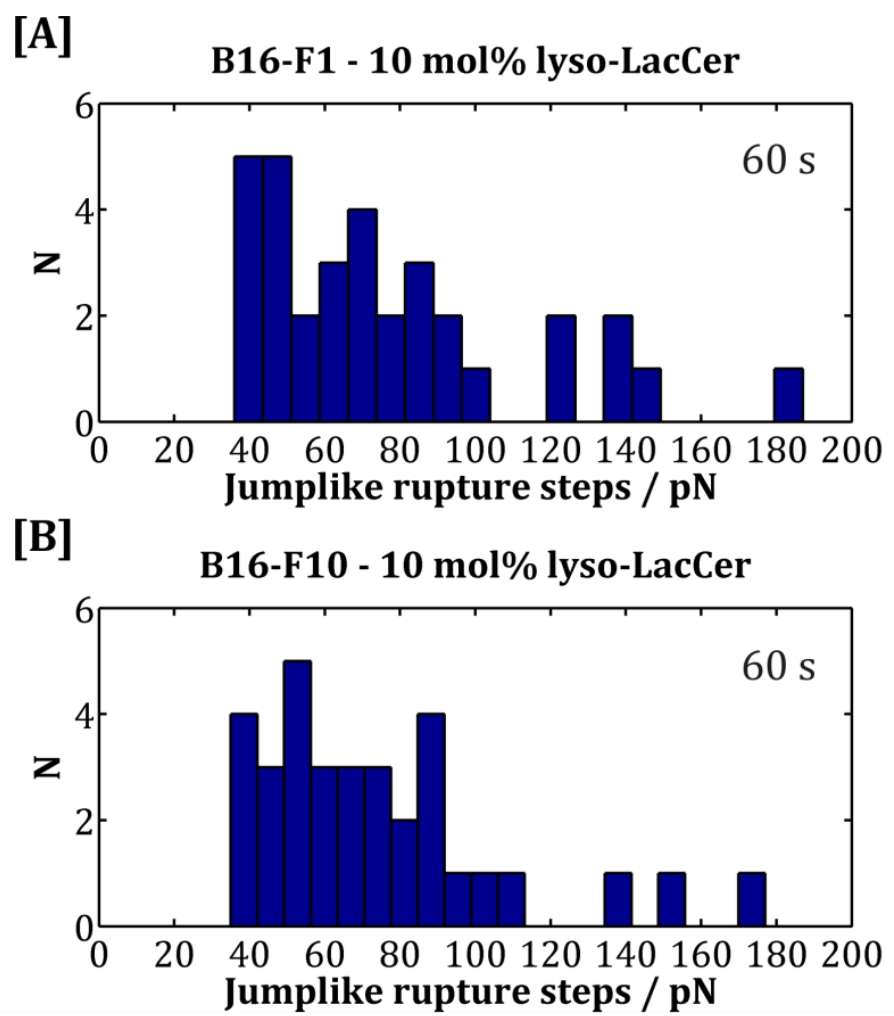

Figure 6.3: Histograms of exemplary jumplike rupture steps obtained in force-distance curves of the retraction process by single-cell force spectroscopy studies. The histograms show the distribution of jumplike rupture steps resulting from the interaction between [A] B16-F1 as well as [B] B16-F10 and 10 mol\% lyso-LacCer-doted SSLMs at contact times of $60 \mathrm{~s} . N=33$ for each histogram. 


\subsection{DISTRIBUTION OF MAXIMUM ADHESION FORCES OF B16-F1}

[A]

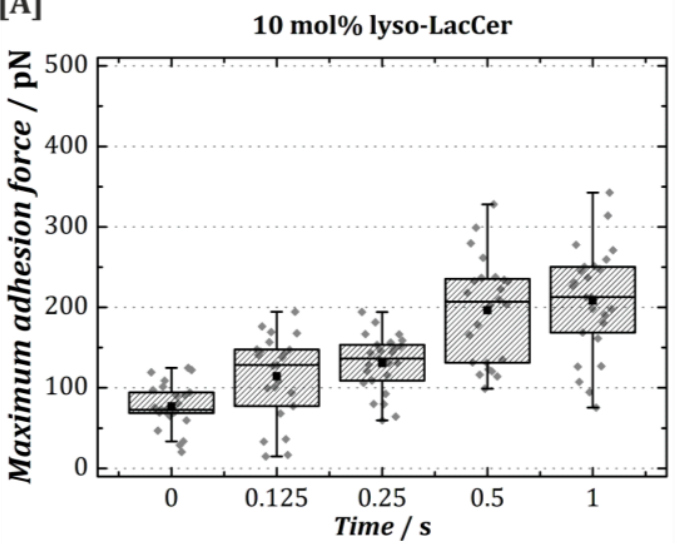

[B]

$1 \mathrm{~mol} \%$ lyso-LacCer

[C]
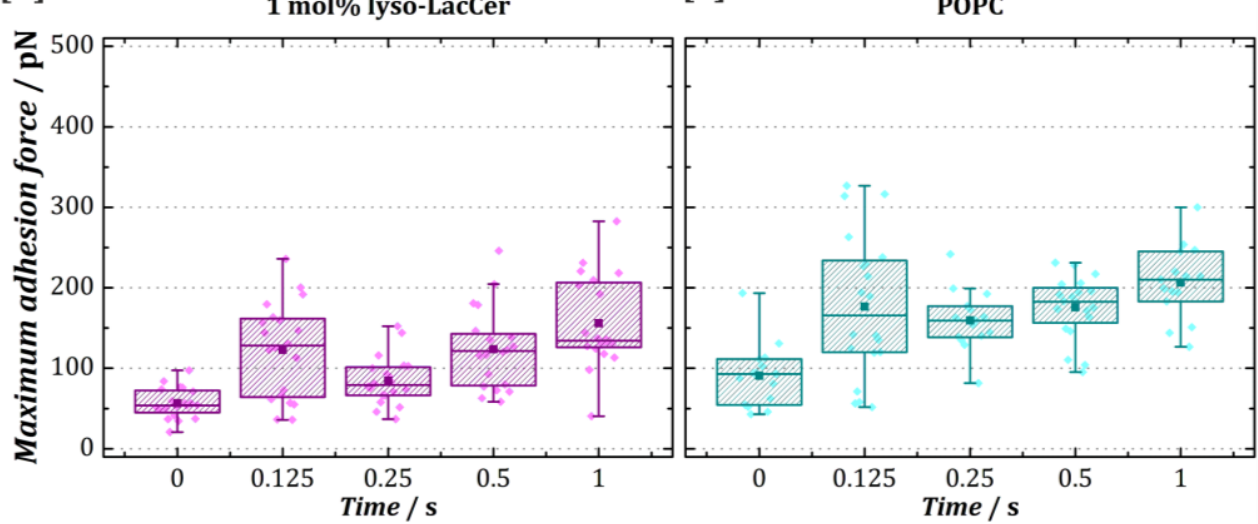

[D]

[E]
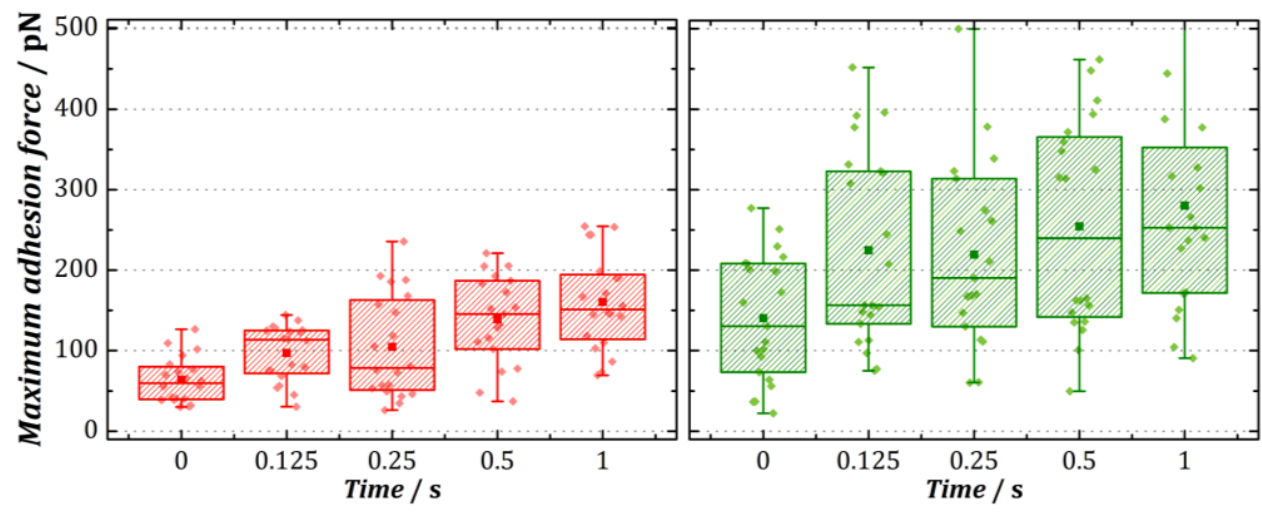

Figure 6.4: Representation of the distribution of the maximum adhesion forces between B16-F1 cells and the respective substrate obtained at contact times smaller than $1 \mathrm{~s}$ with single-cell force spectroscopy. For the measurement of the $\mathrm{G}_{\mathrm{M} 3}$-lyso-LacCer adhesion forces, untreated single cells were pressed onto artificial lipid membranes containing either $10 \mathrm{~mol} \%[\mathrm{~A}]$ or $1 \mathrm{~mol} \%[\mathrm{~B}]$ of the binding partner lyso-LacCer. To prove that cell adhesion was initiated by the interaction between $\mathrm{G}_{\mathrm{M} 3}$ and lyso-LacCer, control measurements were performed. For pure POPC membranes $[\mathrm{C}]$, no binding partners for $\mathrm{G}_{\mathrm{M} 3}$ were available. In further control measurements, the individual cells were first treated with a specific anti- $\mathrm{G}_{\mathrm{M}}$ antibody [D] or the enzyme sialidase [E] and pressed on $10 \mathrm{~mol} \%$ lyso-LacCer-containing membranes. Here, $50 \%$ of all data points are represented by a box containing the median (solid line) and the mean (illustrated by squares in the respective color). The box is limited by an upper (75\% threshold) and lower (25\% threshold) quartile. Data points outside of the box, whose values are limited to 
1.5 times the box length ${ }^{\mathbf{1 7 7}}$, are described by whiskers. Values, which are not within the whisker range, are referred to as outliers. Measurements include about 5 cells and 15-25 force curves per category.

\subsection{DISTRIBUTION OF MAXIMUM ADHESION FORCES OF B16-F1}

[A]
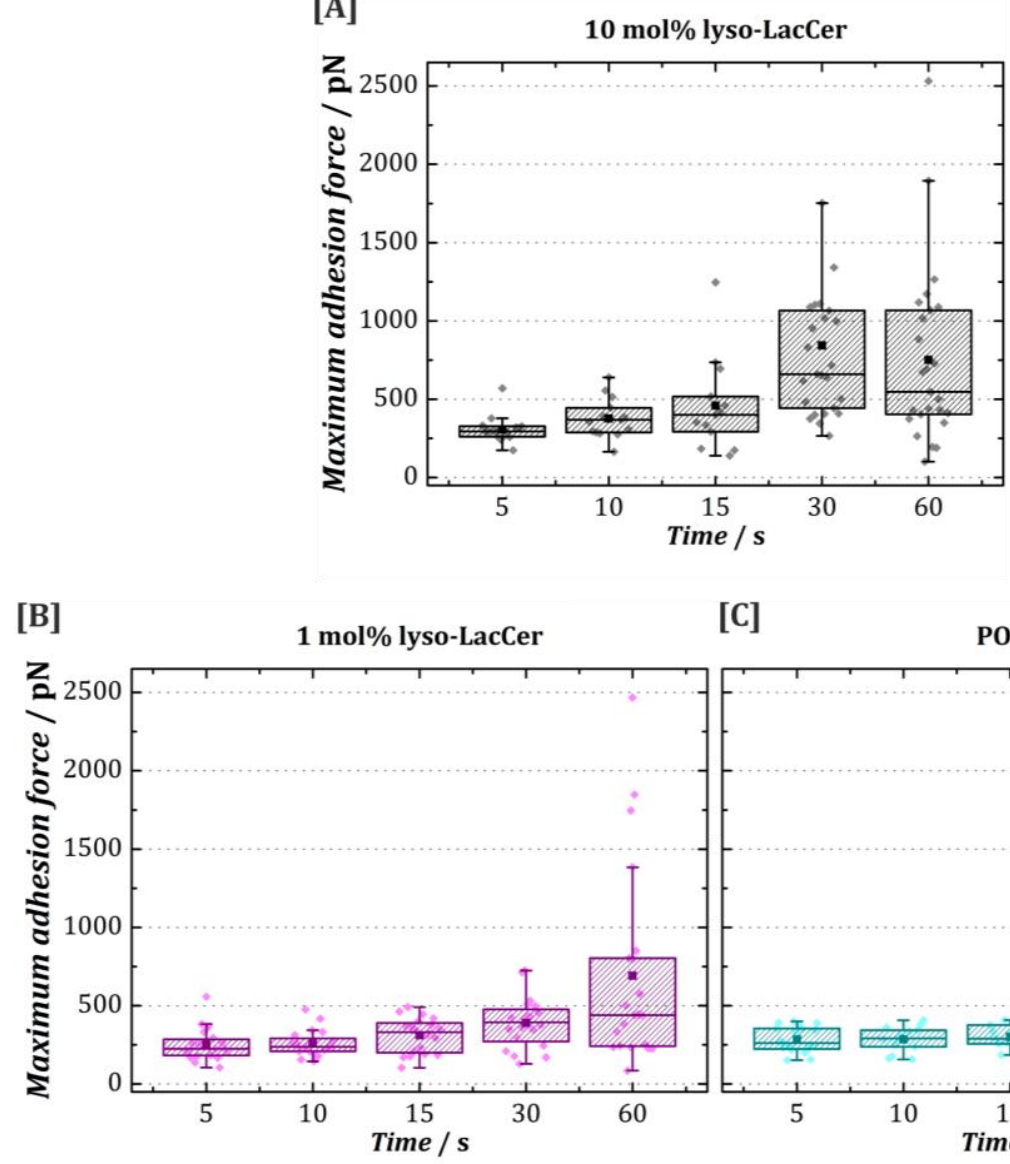

[C]
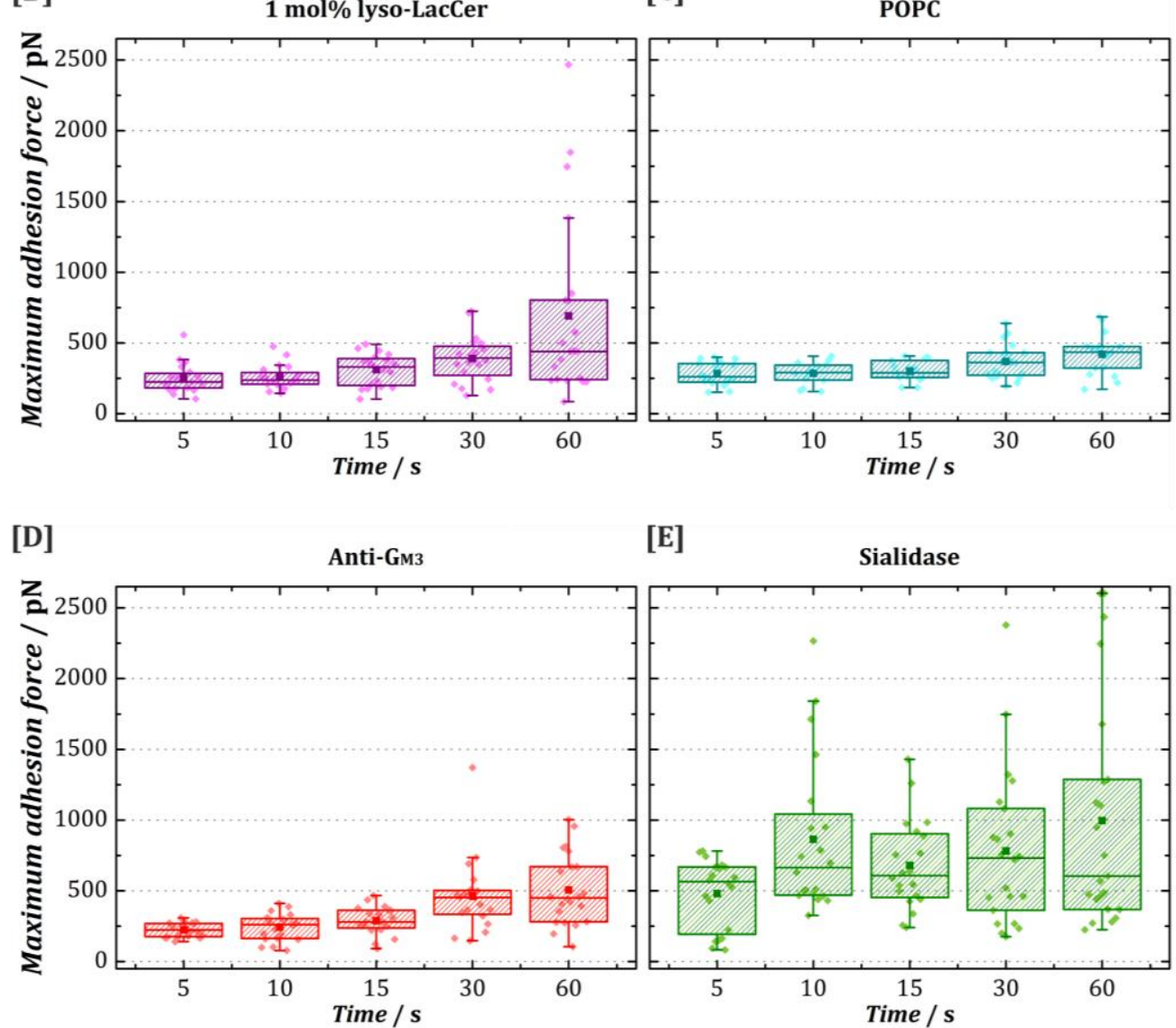

[E]

Sialidase

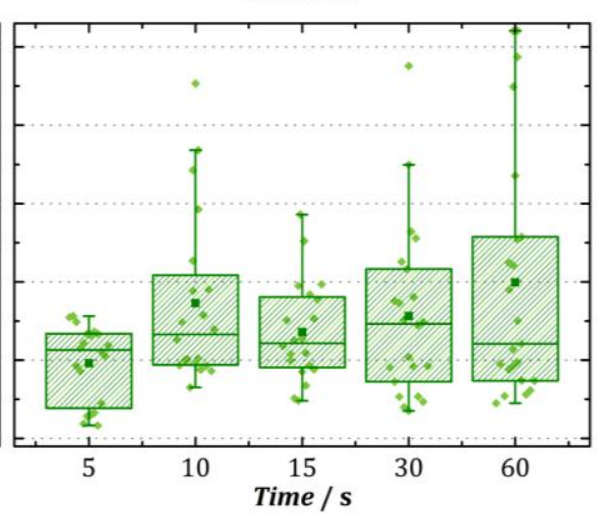

Figure 6.5: Representation of the distribution the maximum adhesion forces between B16-F1 cells and the respective substrate obtained at contact times ranging from 5-60 s with single-cell force spectroscopy. As already described in Figure 3.13, the interaction between $\mathrm{G}_{\mathrm{M} 3}$ and its binding partner lyso-LacCer is represented by the contact of untreated single cells onto artificial lipid membranes containing either $10 \mathrm{~mol} \%$ [A] or $1 \mathrm{~mol} \%$ [B] of lyso-LacCer. For control measurements, untreated cells were brought in contact with pure POPC membranes [C] to ensure that no binding partners were available to $\mathrm{G}_{\mathrm{M} 3}$. For further control measurements, the individual cells were first treated with a specific anti-Gм3 antibody [D] and the enzyme sialidase [E] and pressed on 10 mol\% lyso- 
LacCer-containing membranes. Here, $50 \%$ of all data points are represented by a box containing the median (solid line) and the mean (illustrated by squares in the respective color). The box is limited by an upper (75\% threshold) and lower (25\% threshold) quartile. Data points outside of the box, whose values are limited to 1.5 times the box length ${ }^{177}$, are described by whiskers. Values, which are not within the whisker range, are referred to as outliers. Measurements include about 5 cells and 15-25 force curves per category.

\subsection{LIST OF FIGURES}

Figure 1.1: Schematic representation of the three skin layers referred to as epidermis, dermis and subcutis

Figure 1.2: Phase-contrast micrographs of sub-confluent grown B16-F1 [A] and B16F10 [B] cells. 3

Figure 1.3: Schematic representation of the assumed lipid rafts in the extracellular plasma membrane 4

Figure 1.4: Overview of common sphingolipid structures............................................. 6

Figure 1.5: Minimum-energy model of the GSL Gb5 ....................................................... 7

Figure 1.6: Schematic representation of GSL-enriched microdomains (GEMs, orange) within the extracellular leaflet of a plasma membrane.

Figure 1.7: Schematic illustration of molecular forces between carbohydrate units in GSLs on opposing cellular plasma membranes.

Figure 2.1: Chemical structure of 1-Palmitoyl-2-oleoyl-sn-glycero-3-phosphocholine

Figure 2.2: Chemical structure of D-Lactosyl- $3-1,1^{\prime}-N$-palmitoyl-D-erythrosphingosine .20

Figure 2.3: Chemical structure of D-Lactosyl-ß-1-1'-D-erythro-sphingosine..... .21

Figure 2.4: Representative chemical structure of $\mathrm{G}_{\mathrm{M} 3}$ 21

Figure 2.5: Chemical structure of 2-(4,4-Difluoro-5-methyl-4-bora-3a,4a-diaza-sindacen-3-dodecano-yl)-1-hexadodecanoyl-sn-glycero-3-phosphocholine . .22

Figure 2.6: Chemical structure of Texas Red 1,2-Dihexadecanoyl-sn-glycero-3phosphoethanolamin . 23

Figure 2.7: Schematic drawings of differential scanning calorimetry. 26 
Figure 2.8: Schematic illustration of lipid membranes showing different lamellar phase behavior 29

Figure 2.9: Schematic drawing of an atomic force microscope 32

Figure 2.10: Schematic illustration of a force-distance curve during the retraction process 33

Figure 2.11: Schematic drawing of a single-cell force spectroscopy set-up 35

Figure 2.12: Schematic drawing of a colloidal probe microscopy set-up....... 40

Figure 2.13: Schematic illustration of the two basic measurement principles using imaging atomic force microscopy 44

Figure 2.14: Illustration of a Jablonski diagram 45

Figure 2.15: Schematic illustration of an epifluorescence microscope 47

Figure 2.16: Schematic illustration of a confocal scanning laser microscope 48

Figure 2.17: Schematic illustration of the physical basis of epifluorescence and TIRF microscopy 52

Figure 3.1: Confocal laser scanning microscopy images of sub-confluent grown B16F1 [A] and B16-F10 [B] cell layers 60

Figure 3.2: Total internal reflection fluorescence images of typically shaped single B16-F1 [A, A'] and B16-F10 [B, B'] cells.

Figure 3.3: Exemplary image based cytometry results to investigate the total amount of $\mathrm{G}_{\mathrm{M} 3}$ expressed on the surface of the B16-F1 cells 62

Figure 3.4: Fluorescence-based cytometry analysis of the $\mathrm{G}_{\mathrm{M} 3}$ amount, expressed on the surface of the B16-F1 (green) and B16-F10 (red) carcinoma cell line.

Figure 3.5: Reflection interference contrast microscopy images of separated murine B16 cancer cells grown on different substrates.

Figure 3.6: Phase-contrast images of the adhesive behavior of B16-F1 and B16-F10 cancer cells

Figure 3.7: Differential scanning calorimetry studies of LacCer [A] and lyso-LacCer [B] 
Figure 3.8: Images showing time series of a fluorescence recovery after photobleaching (FRAP) experiment on solid supported lipid membranes . .74

Figure 3.9: Exemplary topographical maps (AFM, tapping mode) of solid supported lipid membranes .76

Figure 3.10: Image of a single B16-F10 cell brought in contact with a 1 mol\% lysoLacCer-containing lipid membrane for $45 \mathrm{~s}$ . .78

Figure 3.11: Exemplary force-distance curves recorded with single-cell force spectroscopy . .80

Figure 3.12: Mean maximum adhesion forces between the B16-F1 and B16-F10 cell line and the respective substrate in dependency of a given contact time ranging from 0-60 s .82

Figure 3.13: Representation of the distribution of the maximum adhesion forces between B16-F10 and B16-F1 cells and the respective substrate obtained at contact times smaller than $1 \mathrm{~s}$ with single-cell force spectroscopy . .83

Figure 3.14: Representation of the maximum adhesion forces between B16-F10 and B16-F1 cells and the respective substrate obtained at contact times ranging from 5-60 s with single-cell force spectroscopy. .84

Figure 3.15: Image of a borosilicate glass microsphere glued to a cantilever tip for colloidal probe microscopy. .88

Figure 3.16: Exemplary force-distance curves of the retraction process recorded by colloidal probe microscopy. .90

Figure 3.17: Histograms of maximum adhesion forces obtained by colloidal probe microscopy measurements

Figure 6.1: Images showing time series of a fluorescence recovery after photobleaching (FRAP) experiment on solid supported lipid membranes V

Figure 6.2: Topographic maps (AFM, tapping mode) of lipid membranes. ..VI

Figure 6.3: Histograms of exemplary jumplike rupture steps obtained in forcedistance curves of the retraction process by single-cell force spectroscopy studies VII 
Figure 6.4: Representation of the distribution of the maximum adhesion forces between B16-F1 cells and the respective substrate obtained at contact times smaller than $1 \mathrm{~s}$ with single-cell force spectroscopy. VIII

Figure 6.5: Representation of the distribution the maximum adhesion forces between B16-F1 cells and the respective substrate obtained at contact times ranging from 5-60 s with single-cell force spectroscopy IX

\subsection{LIST OF TABLES}

Table 2.1: Overview of the composition and ingredients of media and buffer systems used for cell experiments 14

Table 2.2: Overview of buffer system used to establish solid supported lipid membranes

Table 2.3: Overview of lipid mixtures used to establish solid supported lipid membranes

Table 2.4: Overview of the solubility and stock solution concentrations $c$ of the used glycosphingolipids. 24

Table 2.5: Overview of parameters used in DSC experiments 28

Table 2.6: Overview of parameters used for [A] cantilever calibration, [B] attaching a single cell to the cantilever and $[\mathrm{C}]$ obtaining adhesion forces from the single cell with the individual SSLMs for SCFS experiments 38

Table 2.7: Overview of solid supported lipid membranes on glass microspheres and glass substrates for control experiments using colloidal probe microscopy.

Table 2.8: Overview of parameters used for [A] cantilever calibration and [B] obtaining adhesion forces between the GSLs GM3 and lyso-LacCer for CPM experiments

Table 2.9: Overview of filter cube sets used within fluorescent microscopy 47

Table 3.1: Overview of diffusion coefficients $D$ and mobile fractions $F_{\mathrm{m}}$ of various membranes in calcium- or EDTA-containing buffer 75 
Table 3.2: Overview of contact areas of untreated or treated B16-F1 and B16-F10 cells

\subsection{LIST OF SYMBOLS AND ABBREVIATIONS}

\begin{tabular}{|c|c|}
\hline${ }^{\circ} \mathrm{C}$ & Degree Celsius \\
\hline AFM & Atomic force microscopy \\
\hline ATCC & American Type Culture Collection \\
\hline Bodipy $^{\mathrm{TM}}$ & 4,4-Difluoro-4-bora-3a,4a-diaza-s-indacene \\
\hline $\begin{array}{l}\beta \text {-Bodipy }{ }^{\mathrm{TM}} \mathrm{C}_{12} \text {-HPC / } \\
\beta \text {-Bodipy }\end{array}$ & $\begin{array}{l}\text { 2-(4,4-Difluoro-5-methyl-4-bora-3a,4a-diaza-s-indacen-3- } \\
\text { dodecanoyl)-1-hexadodecanoyl-sn-glycero-3-phospho- } \\
\text { choline }\end{array}$ \\
\hline BSA & Bovine serum albumin \\
\hline$c$ & Concentration \\
\hline $\mathrm{c}$ & Centi \\
\hline $\mathrm{Ca}^{2+}$ & Calcium \\
\hline $\mathrm{CaCl}_{2}$ & Calcium chloride \\
\hline $\mathrm{CCI}(\mathrm{s})$ & Carbohydrate-carbohydrate interaction(s) \\
\hline CD9 & Tetraspanin-29 \\
\hline $\mathrm{cf}$ & Confer \\
\hline CLSM & Confocal laser scanning microscopy \\
\hline $\mathrm{CPI}(\mathrm{s})$ & Carbohydrate-protein interaction(s) \\
\hline СРM & Colloidal probe microscopy \\
\hline $\mathrm{cSrc}$ & Proto-oncogene tyrosine-protein kinase \\
\hline $\mathrm{CTB}(\mathrm{s})$ & Cholera toxin B subunit(s) \\
\hline$d$ & Distance \\
\hline$D$ & Diffusion coefficient \\
\hline $\mathrm{Da}$ & Dalton \\
\hline DAPI & 4',6-diamidino-2-phenylindole \\
\hline DEM & Detergent-resistant membrane \\
\hline DMEM & Dulbecco's modified eagle medium \\
\hline DNA & Deoxyribonucleic acid \\
\hline
\end{tabular}


DSC Differential scanning calorimetry

$\Delta$

e

ECM

EDTA

EGF

EGFR

F

$\tilde{F}$

FAK

FBS

FCS

FGF

$F_{\mathrm{m}}$

FRAP

FSC

g

$\operatorname{GEM}(s)$

GFP

GPI

GSL(s)

GUV(s)

$\mathrm{h}$

$\mathrm{H}_{2} \mathrm{O}_{2}$

$\mathrm{HCl}$

HEPES

HOMO

HSA

$\mathrm{Hz}$

$I_{\text {eq }}$
Delta

Elementary charge

Extracellular matrix

Ethylenediaminetetraacetic acid

Epidermal growth factor

Epidermal growth factor receptor

Force

Median fluorescence intensity

Focal adhesion kinase

Fetal bovine serum

Fluorescence correlation spectroscopy

Fibroblast growth factor

Mobile fraction

Fluorescence after recovery photobleching

Forward scatter

Gramm, gravity

GSL-enriched microdomain(s)

Green fluorescent protein

Glycosylphosphatidylinositol

Glycosphingolipid(s)

Giant unilamellar vesicle(s)

Hour

Hydrogen peroxide

Hydrochloric acid

2-[4-(2-hydroxyethyl)piperazin-1-yl]-ethanesulfonic acid Highest occupied molecular orbital

Human serum albumin

Hertz

Fluorescence intensity of the bleached ROI 


\begin{tabular}{|c|c|}
\hline$I_{\mathrm{i}}$ & Fluorescence intensity before bleaching \\
\hline$I(\mathrm{t})$ & Fluorescence intensity at the respective time $t$ \\
\hline$I_{0}$ & Fluorescence intensity after bleaching \\
\hline$I_{1}$ & Fluorescence intensity of the recovered fraction \\
\hline $\operatorname{IgG}$ & Immunoglobulin G \\
\hline $\operatorname{IgM}$ & Immunoglobulin M \\
\hline $\mathrm{k}$ & Kilo \\
\hline$k_{\mathrm{c}}$ & Spring constant \\
\hline $\mathrm{KCl}$ & Potassium chloride \\
\hline $\mathrm{KH}_{2} \mathrm{PO}_{4}$ & Potassium dihydrogenphosphate \\
\hline $\mathrm{L}$ & Liter \\
\hline LacCer & Lactosylceramide \\
\hline$l_{\mathrm{d}}$ & Liquid-disordered phase \\
\hline$l_{0}$ & Liquid-ordered phase \\
\hline LUMO & Lowest unoccupied molecular orbital \\
\hline LUV(s) & Large unilamellar vesicle(s) \\
\hline Lyso-LacCer & Lyso-Lactosylceramide \\
\hline$l_{\alpha}$ & liquid crystalline phase \\
\hline$l_{\beta}^{\prime}$ & gel phase \\
\hline$\lambda$ & Wavelength \\
\hline$\lambda_{\mathrm{BS}}$ & Wavelength beam splitter \\
\hline$\lambda_{\mathrm{em}}$ & Wavelength emission \\
\hline$\lambda_{\mathrm{ex}}$ & Wavelength extinction \\
\hline$M$ & Molar mass \\
\hline M & Molar \\
\hline $\mathrm{m}$ & Milli, meter \\
\hline$m$ & Mass \\
\hline $\min$ & Minute \\
\hline MIP-2 & Macrophage inflammatory protein-2 \\
\hline $\operatorname{MLV}(s)$ & Multilamellar vesicle(s) \\
\hline
\end{tabular}




\begin{tabular}{|c|c|}
\hline mol & Mole \\
\hline$\mu$ & Micro \\
\hline $\mathrm{n}$ & Nano \\
\hline$n$ & Refractive index \\
\hline$N$ & Amount \\
\hline $\mathrm{N}$ & Newton \\
\hline NA & Numerical aperture \\
\hline $\mathrm{Na}_{2} \mathrm{HPO}_{4}$ & Sodium hydrogen phosphate \\
\hline $\mathrm{NaCl}$ & Sodium chloride \\
\hline $\mathrm{NH}_{3}$ & Ammoniac \\
\hline No & Number \\
\hline $\mathrm{O}_{2}$ & Oxygen \\
\hline$\omega$ & Radius \\
\hline $\mathrm{P}$ & Passage \\
\hline PBS & Phosphate buffered saline \\
\hline PDGF & Platelet-derived growth factor \\
\hline PDL & Poly-D-lysine \\
\hline PFA & Paraformaldehyde \\
\hline PMCA & Plasma membrane $\mathrm{Ca}^{2+}$ ATPase \\
\hline POPC & 1-Palmitoyl-2-oleoyl-sn-glycero-3-phosphocholine \\
\hline $\operatorname{PPI}(\mathrm{s})$ & Protein-protein interaction(s) \\
\hline $\operatorname{PRR}(s)$ & Pattern recognition receptors \\
\hline PU & Passage unknown \\
\hline$p_{\beta}^{\prime}$ & Ripple phase \\
\hline $\mathrm{R}$ & Alkyl residue of fatty acid \\
\hline RICM & Reflection interference contrast microscopy \\
\hline ROI & Region of interest \\
\hline s & Second \\
\hline S & Sample \\
\hline SCFS & Single-cell force spectroscopy \\
\hline
\end{tabular}




\begin{tabular}{|c|c|}
\hline $\mathrm{SiO}_{2}$ & Silicon dioxide \\
\hline SM & Sphingomyelin \\
\hline$S_{\mathrm{o}}$ & Solid-ordered phase \\
\hline SSC & Side scatter \\
\hline $\operatorname{SSLM}(\mathrm{s})$ & Solid supported lipid membrane(s) \\
\hline STM & Scanning tunneling microscopy \\
\hline SUV & Small unilamellar vesicle \\
\hline$T$ & Temperature \\
\hline Texas Red ${ }^{\mathrm{TM}}$ & Sulforhodamine 101 acid chloride \\
\hline Texas Red"M DHPE & $\begin{array}{l}\text { Texas Red }{ }^{\mathrm{TM}} \text { 1,2-dihexadecanoyl-sn-glycero-3-phospho- } \\
\text { ethanolamine }\end{array}$ \\
\hline$T_{\mathrm{m}}$ & Main phase transition temperature \\
\hline TRIS & Tris(hydroxymethyl)aminomethane \\
\hline$\tau_{\mathrm{D}}$ & Diffusion time \\
\hline $\mathrm{U}$ & Unit activity enzyme \\
\hline UV & Ultraviolet \\
\hline vol & Volume \\
\hline$w / v$ & Weight/volume \\
\hline$Z_{\mathrm{C}}$ & Cantilever deflection \\
\hline$\Theta$ & Incidence angle \\
\hline$\Theta_{\mathrm{c}}$ & Critical angle \\
\hline
\end{tabular}




\section{DANKSAGUNG}

Von ganzem Herzen möchte ich mich hiermit bei all denjenigen bedanken, die mich auf meinem Weg begleitet, unterstützt und motiviert haben.

Zunächst möchte ich mich bei Dr. Angelika Kunze für die Bereitstellung des interessanten Themas, für die Diskussionsbereitschaft und Hilfestellung bei Problemen bedanken. Durch deine unkomplizierte Art habe ich in den ersten Jahren meiner Doktorarbeit viel von dir lernen können. Danke, dass du mir den Freiraum und das Vertrauen gegeben hast, meinen eigenen Weg zu gehen und eigene Ideen zu entwickeln. Danke an dieser Stelle auch für das Korrekturlesen meiner Arbeit. Für deinen neuen Lebensweg wünsche ich dir alles Gute!

Weiterhin möchte ich mich bei Prof. Dr. Andreas Janshoff für die unproblematische und sofortige Übernahme meiner Betreuung in den letzten zwei Jahren bedanken, so dass ich meine Arbeit fertig stellen konnte. Vielen Dank für die fachlichen Ratschläge, Tipps und das Korrekturlesen meiner Arbeit.

Auch möchte ich mich bei Prof. Dr. Claudia Steinem und Dr. Florian Rehfeldt bedanken, die mich im Rahmen der Thesis-Commitee-Treffen durch die hilfreichen Diskussionen tatkräftig unterstützt haben. Ein Danke an dieser Stelle auch an die weiteren Mitglieder meiner Prüfungskommission: Dr. Sebastian Kruss, Prof. Dr. Rizzoli und Prof. Dr. Michael Meinecke.

Ein ganz besonderes Dankeschön geht an die Mitglieder des Arbeitskreises Janshoff. Durch die herzliche Aufnahme gleich vom ersten Tag an, habe ich mich immer sehr wohl bei euch gefühlt und konnte durch die unzähligen Kaffeepausen, Bastel-, Grillund Filmabende wieder Kraft tanken. Vielen Dank vor allem an Filip, Ilyas, Jonathan und Stefan dafür, dass ich mich stets bei euch verstecken, mich freuen und schimpfen durfte (der Harry-Potter-Raum deprimiert ja auch mit der Zeit). Zu sportlichen 
Höchstleistungen habt ihr mir verholfen: Helen, Ilyas und Jonathan, Actin in Action, meine Laufcrew! Susi und Filip, ihr seid fantastische Fans! Marian, dir danke ich für die großartige Unterstützung im Bereich Lehre und das damit verbundene stundenlange Korrekturrechnen der Klausuren! Hannes, ich bedanke mich für das Grübeln über meine Messergebnisse, für deine stetige Hilfsbereitschaft bei Hemmschuhen aller Art, die Unterstützung im Labor und das aufmerksame Korrekturlesen meiner Arbeit. Petra, ein großes Dankeschön dafür, dass du mich im administrativen Chaos nicht hast untergehen lassen und immer das richtige Formular aus deinem Schreibtisch zaubern konntest. Angela, Tabea und Anja, ohne eure großartige Arbeit im Zellkulturlabor hätte ich meine Experimente und diverse Praktika nicht meistern können, danke für die hervorragende Unterstützung. Tabea, danke auch dir für deine Korrekturvorschläge. Anja, dir danke ich zusätzlich für die lustigen Kaffee- und Mittagspausen und spontanen Besuche, sie haben mir stets den Alltag versüßt! Zudem möchte ich mich bei meinen Bachelor- und Masterstudenten sowie Praktikanten bedanken, die mir fleißig bei der Bewältigung der Arbeit geholfen haben.

An Susi, meine tolle Schreibtischnachbarin, und meine liebe Katja: ich danke euch für einfach alles! Für euren Beistand, euer offenes Ohr und für eure unermüdliche Motivation von nah und fern. Susi, mit deiner gewissenhaften Korrektur meiner Arbeit (selbst an deinen freien Tagen!) sowie durch die fachlichen und privaten Gespräche hast du sehr zum Gelingen meiner Arbeit beigetragen. Danke!

Lisa, Sabine, Karina, Imke und Anna! Einfach nur ein großes Dankeschön an euch und eure Freundschaft über all die Jahre. Auch wenn wir uns leider nicht oft sehen, weiß ich doch, dass ich mich immer auf euch verlassen kann. Mit euch ist das Leben einfach viel lebenswerter und vor allem lustiger!

Meiner Familie möchte ich ganz besonders für die nie enden wollende Unterstützung und Liebe danken! Es tut gut zu wissen, dass ihr immer für mich da seid, mich auffangt und mir den Weg ebnet, wenn es mal steinig wird. Ich hoffe ihr wisst, was ihr mir bedeutet!

Markus, dich an meiner Seite zu wissen ist das größte Geschenk. Ich danke dir von ganzem Herzen dafür, dass du mich begleitest und mich auch in stürmischen Zeiten immer wieder zum Lachen bringst. Durch dich weiß ich, dass ich alles schaffen kann und dass meine Welt in deinen Händen sicher ist. Ich freue mich auf unsere weitere gemeinsame Reise und auf alles, was kommt. Danke! 